a Ph.D. dissertation on

Transcranial Ultrasound Holograms for the Blood-Brain Barrier Opening

Sergio Jiménez Gambín 


\title{
Transcranial Ultrasound Holograms for the Blood-Brain Barrier Opening
}

\author{
Sergio Jiménez Gambín
}

A thesis submitted in partial fulfillment of the requirements for the degree of

Doctor of Philosophy

Dissertation supervisors:

Francisco Camarena

Universitat Politècnica de València, Spain

Noé Jiménez

Universitat Politècnica de València, Spain

Dissertation jury:

Jonathan Vappou

José Ángel Obeso

Centre National de la Recherche Scientifique, France

Peer Fischer

CINAC Hospitales de Madrid, Spain

University of Stutgart, Germany

Dissertation reviewers:

Jonathan Vappou

José Ángel Pineda

Hermes Kamimura
Centre National de la Recherche Scientifique, France CINAC Hospitales de Madrid, Spain Columbia University, USA

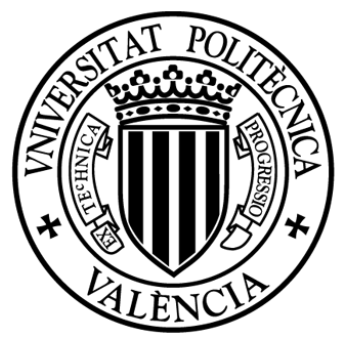

Instituto de Instrumentación para Imagen Molecular (i3M)

Universitat Politècnica de València, Spain

June 2021 



\section{Acknowledgments}

The story of how I ended up doing this thesis starts with Prof. Jaime Ramis. He was my teacher in the last year of my BSc in Sound and Image in Telecommunication Engineering at Universidad de Alicante (UA). Then, he organized a group visiting trip to the Escuela Politécnica Superior, in Gandía city, at Universidad Politécnica de Valencia (UPV), where he had previously worked in, to show us the wide variety of research lines they had. It was then when I discovered the research line on ultrasound applied to medicine. Thereby, Jaime deserves my very first and kind acknowledgment.

Later, searching the internet for more information on this area about medical ultrasound research at UPV, I found a video interview of a young researcher talking about the use of ultrasound to open the blood-brain barrier for the treatment of Alzheimer's Disease. This video changed my life. My granny Antonia suffered from Alzheimer's, and at that right time that I watched that video, I knew I wanted to dedicate my time and effort to find a solution to help Alzheimer's patients.

The guy who was talking in that interview was the $\mathrm{PhD}$ student Noé Jiménez (currently Dr.), whose thesis director was Prof. Francisco Camarena, Paco for friends. I immediately contacted Paco and my course began to take an unstoppable and clear direction.

After finishing my BSc Degree, I started a MSc in Acoustic Engineering at UPV with Prof. Paco as supervisor, who later offered me a research internship to start my $\mathrm{PhD}$ candidature at his recently established laboratory at UPV but in Campus de Vera, in Valencia city. Since Paco's new laboratory was in its early development stage, funding resources and, 
therefore, research equipments were scarce, which led to a hard beginning of my thesis. However, Paco, Noé and I worked hard to get a $\mathrm{PhD}$ funding grant from Generalitat Valenciana, and through the years, the excellent work of Paco has led to lots of funding for our laboratory and new researchers have joined us; from four people at the beginning, to fifteen nowadays. For all this, and more than deserved, I acknowledge my primary director and friend Paco from the bottom of my heart because he's always been the light illuminating my steps and making them possible.

Dr. Noé, who became my second director and the key piece of this puzzle, brought to our laboratory the idea of acoustic holography as a revolutionary approach for biomedical applications. His knowledge and experience on acoustics were essential for me to really understand the behavior of mechanical waves, I really learned and enjoyed it, and after a hard research work, we finally were able to develop and patent this novel approach based on 3D-printed holographic acoustic lenses, fact which gave our laboratory a remarkable push. Also note that his mentoring during my research stay at Université du Maine (France) was brilliant.

Noé, I've always said of you that you are a genius, and you know it, my friend. Thereby, for all your valuable mentoring, my most sincere acknowledgment, because without you, I would not have given birth to such a powerful and beautiful thesis.

Acknowledgments to Prof. José María Benlloch, who is the director of the Instituto de Instrumentación para Imagen Molecular (i3M), the department where I developed my thesis, for his support, long career, and professional experience in research.

Acknowledgments to the Spanish institution Generalitat Valenciana, which funding grant allowed me to develop this doctoral thesis, and as well funded my research stay at Columbia University. The development of the entire thesis was supported through grant No. ACIF/2017/045. Particularly, the research carried out in Chapter 3 and Chapter 4 was possible thanks to and supported through grant BEFPI/2019/075. Action co-financied by the Agència Valenciana de la Innovació through grant INNVAL10/19/016 and by the European Union through the Programa Operativo del Fondo Europeo de Desarrollo Regional (FEDER) of the 
Comunitat Valenciana 2014-2020 (IDIFEDER/2018/022).

Regarding the research stay, and since Noé mentioned it in his interview about ultrasound applied to Alzheimer's, I had always been dreaming of the day when I could join that collaboration with the UEIL (Ultrasound Elasticity Imaging Laboratory) at Columbia University, in New York city, led by Prof. Elisa Konofagou. Therefore, my honest acknowledgment to Prof. Elisa for accepting our research proposal and, thus, for giving us the opportunity to demonstrate that acoustic holograms do work in vivo. Also acknowledge Dr. Antonios Pouliopoulos for his excellent mentoring during my research stay at UEIL. Finally, acknowledgments to Dr. Cheng-Chia $W u$, who I met during my last week of stay, for enabling such a beautiful collaboration path on pediatric oncology using acoustic holograms.

To my dear laboratory mates Álvaro, Josep, Miguel, Jandri and Diana, who made funnier and more familiar these years of research, specially conference and laboratory dinners, and helped me with some technical research aspects. To the department mate Koldo, who helped us to 3D-print the first prototypes of the lenses. To my dear research mates María and Rober, who supported and helped me during these years of research. To my dear friends José David and Hirahi, who were very interested in my research work (thanks Dave for your radio interview) and also supported me since I met them in Alicante during my BSc Degree, sois unos cacos.

A mis padres Carmina y Ángel, que me apoyan enormemente desde mis comienzos académicos, tanto económica como emocionalmente, permitiéndome así llegar hasta aquí y acabar realizando esta bonita tesis doctoral. Es un logro y un orgullo no solo para mí, sino para toda nuestra familia. Agradecer también a mi hermano Mario por su apoyo.

Y a Maribel, que apareció en mi vida en el tercer año de esta tesis (gracias a la música), y aún así ha sido un grandísimo apoyo emocional.

Por cierto, he podido comprobar de primera mano que el dicho de que realizar un doctorado te genera adversas pérdidas de memoria jes cierto! Mis más sinceras disculpas porque jos he vuelto locos sin querer! 

Mis últimas palabras a la persona más especial, la que se fue y nunca olvido, la que se fue y ya dejó mi camino escrito, razón por la que yo investigo, para que algo asi no sea nuevamente sufrido.

Tu memoria en este libro, deja huella con cariño, tu recuerdo es mi instinto, y asi, lucharemos unidos, por un mundo libre de olvido.

Gracias a ti, abuelita Antonia, donde quiera que estés, te quiero. 



\section{Abstract}

Treatments for neurological diseases are strongly limited by the inefficient penetration of therapeutic drugs into the diseased brain due to the blood-brain barrier (BBB), and therefore no health improvement can be achieved. In fact, the BBB is a protection mechanism of the human body to avoid the diffusion of potentially dangerous agents into the central nervous system. Nevertheless, this barrier can be successfully inhibited by using a worldwide spread technology based on microbubble-enhanced focused ultrasound. Its main advantage is its non-invasive nature, thus defining a patient-friendly clinical procedure that allows to disrupt the BBB in a safe, local and transient manner. Conventionally, the diseased brain structure has been targeted in its center, with a single focus. However, Alzheimer's or Parkinson's Diseases do require that ultrasound is delivered to entire, complex-geometry and large-volume structures located at both hemispheres of the brain. Therefore, current technology presents several limitations as it does not fulfill these requirements. This doctoral thesis aims to develop a novel technique based on using focused ultrasound acoustic holograms to solve the existing limitations to treat neurological diseases. In this dissertation, we study 3D-printed holographic acoustic lenses coupled to a single-element transducer that allow to accurately control the acoustic wavefront to both (1) compensate distortions suffered by the beam in its path to the brain, and (2) simultaneous focusing in multiple and complex-geometry structures or acoustic vortex generation, providing a time- and cost- efficient procedure. Therefore, the research carried out throughout this thesis opens a promising path in the biomedical field to improve the treatment for neurological diseases, neurostimulation or tissue ablation applications. 



\section{Resumen}

El tratamiento de enfermedades neurológicas está muy limitado por la ineficiente penetración de los fármacos en el tejido cerebral dañado debido a la barrera hematoencefálica (BHE), lo que imposibilita mejorar la salud del paciente. La BHE es un mecanismo de protección natural para evitar la difusión de agentes potencialmente peligrosas para el sistema nervioso central. No obstante, la BHE se puede inhibir mediante ultrasonidos focalizados e inyección de microburbujas de forma segura, localizada y transitoria, una tecnología empleada mundialmente. La principal ventaja es su carácter no invasivo, siendo así muy atractiva y cómoda para el paciente. Normalmente, la zona cerebral enferma se trata en su parte central empleando un único foco. Sin embargo, enfermedades como el Alzheimer o el Parkinson requieren un tratamiento sobre estructuras de geometría compleja y tamaño elevado, situadas en ambos hemisferios cerebrales. Por tanto, la tecnología actual está muy limitada al no cumplir dichos requisitos. Esta tesis doctoral tiene como objetivo el desarrollo de una técnica novedosa, basada en hologramas acústicos, para resolver las limitaciones presentes en los tratamientos neurológicos empleando ultrasonidos. Se estudian las lentes acústicas holográficas impresas en 3D, que acopladas a un transductor mono-elemento, permiten el control preciso del frente de onda ultrasónico tanto para (1) compensar las distorsiones que sufre el haz hasta alcanzar el cerebro, como (2) focalizarlo simultáneamente en regiones múltiples y de geometría compleja o formando de vórtices acústicos, proporcionando así efectividad en tiempo y coste. Por ello, la investigación desarrollada en esta tesis abre un camino prometedor en el campo de la biomedicina que permitirá mejorar los tratamientos neurológicos, además de aplicaciones en neuroestimulación o ablación térmica del tejido. 



\section{Resum}

El tractament de malalties neurològiques està molt limitat per la ineficient penetració del fàrmac en el teixit cerebral danyat a causa de la barrera hematoencefàlica (BHE), i així no és possible una millora de salut del pacient. La BHE és un mecanisme de protecció natural per a evitar la difusió d'agents potencialment perillosos per al Sistema Nervios Central. No obstant això, aquesta barrera es pot inhibir mitjancant una tecnologia emprada mundialment basada en ultrasons focalitzats i injeccio de microbombolles. El principal avantatge és el seu caràcter no invasiu, sent així molt atractiva i còmoda per al pacient, i permet obrir la BHE de manera segura, localitzada i transitòria. Normalment, la zona cerebral malalta es tracta en la seua part central, emprant un unic focus. No obstant això, malalties com l'Alzheimer o el Parkinson requereixen un tractament al llarg d'estructures de geometria complexa i grandària elevada, situades en tots dos hemisferis cerebrals. Per tant, la tecnologia actual està fortament limitada al no complir amb aquests requeriments. Aquesta tesi doctoral està enfocada a investigar i desenvolupar una tècnica nova, basada en hologrames acústics, per a solucionar les limitacions presents en els tractaments neurològics. Una lent acústica holograca de baix cost impresa en 3D acoblada a un transductor d'element simple permet el control precs del front d'ona ultrasònic punt per a (1) compensar les distorsions que pateix el feix en el seu camí cap al cervell, i (2) focalització simultània del feix en regions multiples i de geometria complexa, proporcionant aix un tractament efectiu en temps i cost. Per això, la investigació desenvolupada en aquesta tesi demostra la possibilitat de realitzar qualsevol tractament neurològic, a més d'aplicacions en la neuroestimulació o l'ablació tèrmica dins del camp biomèdic. 



\section{Contents}

1 Introduction 1

1.1 Brain diseases . . . . . . . . . . . . . . . . 2

1.2 The blood-brain barrier $(\mathrm{BBB}) \ldots \ldots \ldots \ldots$

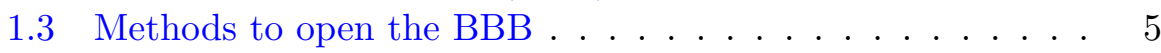

1.3.1 Invasive approaches . . . . . . . . . . . 5

1.3.2 Non-invasive approaches . . . . . . . . . . . . . 5

1.3.3 Focused ultrasound as the promising approach for CNS drug delivery . . . . . . . . . . . . 7

1.4 Thesis motivation . . . . . . . . . . . . . . . . . 9

1.4 .1 Objectives . . . . . . . . . . . . . 10

2 Holograms to focus ultrasonic beams through the human skull $\quad 19$

2.1 Introduction . . . . . . . . . . . . . . . 20

2.2 Methods . . . . . . . . . . . . . . . . 23

2.2.1 Tomographic image acquisition . . . . . . . . . . 24

2.2 .2 Calculation methods . . . . . . . . . . . . 25

2.2 .3 Lens design . . . . . . . . . . . . . . . 27

2.2 .4 Skull phantom . . . . . . . . . . . . . . 31

2.2.5 Measurement setup . . . . . . . . . . . . . . . 32

2.3 Multiple-point holograms . . . . . . . . . . . . . . 33

2.4 Self-bending beams . . . . . . . . . . . . . 35

2.5 Volumetric holograms overlapping CNS structures . . . . . 37

2.6 Hologram simulation using a realistic skull . . . . . . . . . 40

2.7 Conclusions . . . . . . . . . . . . . . . . 45 
3 Validation of acoustic holograms in small laboratory animals

3.1 Introduction . . . . . . . . . . . . . . . 60

3.2 Methods . . . . . . . . . . . . . . . . . 61

3.2.1 Numerical simulation . . . . . . . . . . . . . 62

3.2.2 Mouse skull acquisition for simulation . . . . . . . 63

3.2.3 Lens design . . . . . . . . . . . . . . . . . . 63

3.2 .4 Guiding and monitoring system . . . . . . . . . 65

3.2.5 Sonication and post-MRI procedure . . . . . . . 65

3.2.6 Cavitation signal processing . . . . . . . . . . . 66

3.2.7 BBB opening volume quantification . . . . . . . . 67

3.2 .8 Acoustic calibration setup . . . . . . . . . . 67

3.3 Results . . . . . . . . . . . . . . . . . . 69

3.3.1 System calibration . . . . . . . . . . . . . . 69

3.3.2 Bilateral BBB opening . . . . . . . . . . . . . . 72

3.3 .3 Cavitation analysis . . . . . . . . . . . . . 75

3.4 Conclusions . . . . . . . . . . . . . . . 76

4 Hologram design for targeted chemotherapy $\quad 87$

4.1 Introduction . . . . . . . . . . . . . . . . . 88

4.2 Materials and methods . . . . . . . . . . . . . . . . 89

4.2.1 Numerical simulation . . . . . . . . . . . . . . 89

4.2.2 CT and MRI acquisition . . . . . . . . . . . . 90

4.2 .3 Lens design . . . . . . . . . . . . . . . . . . . . 90 90

4.2.4 Quality parameters evaluation .......... 91

4.2.5 Optimal holographic wavefront definition . . . . . 93

4.3 FDA-approved single-element FUS transducer . . . . . . . . 96

4.4 Bifocal-hologram approach . . . . . . . . . . . . 98

4.5 Volumetric slice-hologram approach . . . . . . . . 100

4.5.1 Alternative slice-hologram approach . . . . . . . . 102

4.5.2 Higher aperture feasibility approach . . . . . . . . . 104

4.5.3 Higher and complete active aperture feasibility approach . . . . . . . . . . . . . 106

4.6 Discussion . . . . . . . . . . . . . . . . . . . . . . . 108

4.7 Conclusion . . . . . . . . . . . . . . . . 110 
5 Transcranial holographic ultrasonic vortices 115

5.1 Introduction . . . . . . . . . . . . . . . 116

5.2 Hologram generation . . . . . . . . . . . . . . . 117

5.3 Results. . . . . . . . . . . . . . . . . 120

5.3.1 Vortices in homogeneous media . . . . . . . . . 120

5.3.2 Vortex synthesis in transcranial propagation . . . . . 122

5.3.3 High-order topological charge transcranial vortex synthesis . . . . . . . . . . . . . . . 125

5.4 Conclusions . . . . . . . . . . . . . . . . . 128

6 Broad depth-of-field Bessel beams using acoustic holograms 137

6.1 Introduction . . . . . . . . . . . . . . . . . . . 138

6.2 Methods . . . . . . . . . . . . . . . . . . 142

6.2.1 Analytical and numerical field calculations . . . . . . 142

6.2.2 Flat-intensity Bessel beams design . . . . . . . . . . 142

6.2.3 Experimental field measurements . . . . . . . . . . . 143

6.3 Generating a Bessel beam . . . . . . . . . . . . . . . . . 144

6.4 Performance of holographic Bessel beams . . . . . . . . . 148

6.5 Broadband behaviour of fraxicons and phase-only holograms 149

6.6 Pixel quantization . . . . . . . . . . . . . . . . 153

6.7 Experimental validation . . . . . . . . . . . . 156

6.7.1 Zeroth-order Bessel beams . . . . . . . . . . . . . . 157

6.7.2 High-order Bessel beams (vortex beams) . . . . . . . 159

6.8 Conclusions . . . . . . . . . . . . . . . . . . 161

7 General discussion and conclusions 171

7.1 Summary and discussion of the proposed approach . . . . 172

7.2 Chapters summary . . . . . . . . . . . . . . . . 174

7.3 Published papers . . . . . . . . . . . . . . . . . . 177

7.4 Unpublished papers . . . . . . . . . . . . . . . . 178

7.5 Conference proceedings . . . . . . . . . . . . . . 179

7.6 Conference awards . . . . . . . . . . . . . . . . 182 




\subsection{Brain diseases}

What makes human beings unique species is their brain. Humans have the cognitive capacity of processing information by means of memory, attention, perception, creativity, abstract or analogical thought. The human nervous system is subdivided into two parts: the central nervous system (CNS), defined by the brain and spinal cord, and the peripheral nervous system (PNS), defined by the cranial and spinal nerves. The largest CNS belongs to human primates $[1,2]$. On the one hand, rodents, such as mice, have about 71 [3] million neurons, and non-human primates, such as chimpanzee and macaque, have about 7.4 [4] and 1.7 [3] billion neurons, respectively, within the cerebral neocortex (brain structure which enables a higher cognitive function in mammals [5]), compared to approximately 16.3 [6] billion neurons of the human brain. However, the ratio between the size of the brain and the mental ability is weak for human primates among mammals [1].

The brain is the control center of our body, protected by the skull; however, it can suffer from many diseases: mental disorders such as depression, post-traumatic stress disorder (PTSD), or schizophrenia; brain injuries such as hematomas, blood clots, or strokes; malignant brain tumors; and neurodegenerative diseases (dementia). Such an essential part of our body should be better understood in healthy and disease conditions where debilitating cognitive and motor impairment, including death, may occur. Three of the most studied brain-related diseases, for the ones many clinical procedures have been developed and approved by the Food and Drug Administration (FDA), are essential tremor (ET), Alzheimer's disease $(\mathrm{AD})$ and Parkinson's disease (PD).

ET is the most common tremor disorder globally, affecting $3 \%$ of the population [7-10], characterized by kinetic tremor of the upper limbs. People older than 40 years old are more susceptible to ET [11]. ET is a tremor that intensifies when an action or voluntary movement is done, i.e. eating or writing, unlike $\mathrm{PD}$, characterized by a resting tremor, not correlated with movement [12]. ET can also manifest in the neck, jaw, or vocal apparatus [13]. Besides this kinetic tremor, other symptoms such as mild ataxia and a variety of non-motor cognitive and psychiatric signs can be relevant [14-16]. Traditional treatments for this progressive disease 
showed poor efficiency, but current technologies using focused ultrasound allow a high-efficient and effective treatment [17-19].

Parkinson's disease (PD) is the second most common neurodegenerative movement disorder, affecting about $3 \%$ of the worldwide population older or equal to 65 years old [20]. Experienced effects are resting tremor, rigidity, postural instability, bradykinesia/akinesia, and other motor and non-motor symptoms [21]. Traditional treatments allow good control of motor symptoms but cannot slow down or stop the progression of neurodegeneration, the increasing disability, and, therefore, the evolution of the disease [22]. The current treatment basis is similar to treatment for ET.

Alzheimer's disease (AD) is the most common neurodegenerative disease, affecting about $4 \%$ of the worldwide population older or equal to 65 years old (a $70 \%$ of $\mathrm{AD}$ dementia within 35.6 million people affected by all-type dementia) $[23,24]$. AD is characterized by early impairment in learning and memory, impairments in complex attention, executive function, language, behavior and/or social comportment [25]. The cognitive function progressively decays as the individuals continue to age [26, 27], and therefore, aging is the most important risk factor related to AD [28]. In addition to aging, other factors such as family history of dementia, genetics, or being female, are risk circumstances in AD [23]. Conventional and current drug-based treatments are limited in terms of perfusion of the drug into the diseased tissue, however, novel technologies using focused ultrasound allow a more effective and efficient manner of distributing the therapeutic drugs into the desired CNS region [29, 30].

\subsection{The blood-brain barrier (BBB)}

Historical review [31] reveals that the first work showing the existence of this particular barrier was reported by Ehrlich more than a century ago [32], where laboratory animals were injected by a chemical substance which penetrated all organs in the body except the CNS. Nowadays, treatments for related-brain diseases are limited by the blood-brain barrier (BBB) [33] because therapeutic drugs cannot penetrate it and reach the diseased brain tissue. Thus, the resulting efficacy and efficiency of the treatment are highly reduced. 
Aside from the skull as a protection element to the brain and taking a closer look at a cellular level, the BBB is a specialized membrane formed by the endothelial cells lining the cerebral microvessels and interconnected with the tight junctions (as sketched in Fig. 1.1), covering the blood capillaries distributed through the brain tissue, whose function is to prevent the passage of substances or molecules potentially dangerous from the bloodstream to the CNS tissue [35-37]. Only $2 \%$ of all small- and none of the large-size molecules cross the BBB [38, 39]. Because of this, and in addition to the few solutions provided by the industry to the BBB problem [40], most CNS disorders are difficult to treat. However, they could be highly benefited by improving the drug therapy. For the therapeutic small-size drugs to efficiently pass through the BBB, the molecules must have both a molecular mass $<400-500$ Da and high lipid solubility [41].

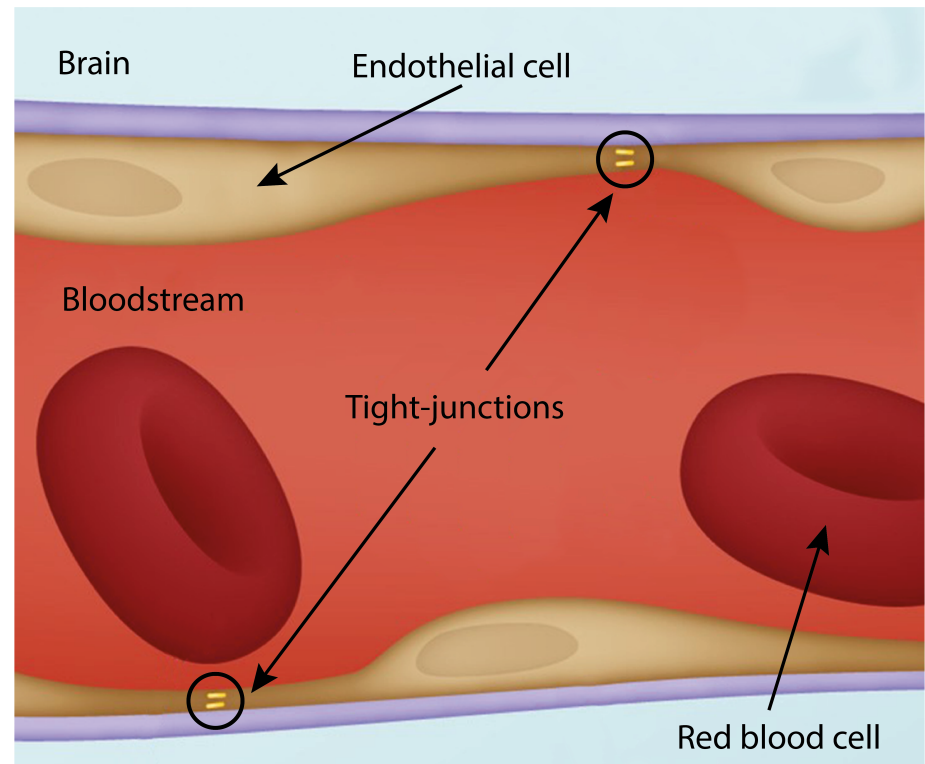

Figure 1.1: The blood-brain barrier, formed by endothelial cells joined by the tight-junctions, separates the brain tissue from the bloodstream as a protection mechanism to maintain the brain healthy from external pathogens which could be harmful for the CNS. Adapted from [34]. 


\subsection{Methods to open the BBB}

Several approaches have been investigated and developed to administrate therapeutic drugs into the CNS, generally classified into invasive and non-invasive [34].

\subsubsection{Invasive approaches}

Intrathecal administration is an invasive method to ensure drug concentration in the brain by direct administration using an intracerebroventricular port implanted under the scalp or into the lumbosacral subarachnoid space of the spinal cord [42].

Convention-enhanced delivery requires a minimally invasive exposure of the brain, followed by the placement of several micro-infusion pumps, so that drugs can consistently concentrate at the target tissue [43].

Interstitial wafers and polymeric implants, placed through the resection cavity in the treatment of glioblastoma [44], show poor drug penetration and limited dosage, in addition to be a method associated with high intracranial pressures and local toxicity causing therefore brain trauma and infections [45].

Furthermore, these invasive approaches, which are more adequate for the treatment of brain tumors as these implants are inserted during a tumor resection through the skull, do require a high cost of maintenance and the necessity of follow-ups, a high capacity of resources is needed, and consequently, they are not adequate to treat brain diseases associated with a long period of treatment such as AD or PD. Generally, the development of non-invasive strategies is key to warrant the success of the treatments for brain diseases.

\subsubsection{Non-invasive approaches}

One approach is to perform a chemical modification of drugs, increasing their lipidization so that the BBB permeability increases and the drug can cross it more efficiently [46]. However, a higher lipidization leads to faster elimination of the molecule from the circulatory system, therefore reducing the drug distribution. Also, if molecules are not target-specific, 
there will be a systemic delivery of drugs, with potential limitations due to dosing and adverse effects.

Another approach is to deliver therapeutic drugs via viral vectors because of their natural ability to infect cells at a high efficiency [47]. However, there is a possible risk of oncogenesis and lethality [48].

Intranasal administration is a method that uses the olfactory and trigeminal nerves in the nasal mucosa to deliver drugs to the brain [49]. However, the administration is generally not performed by a medically trained professional, as well as the degradation of drugs by mucosal enzyme, a short retention time in the nasal cavity, and restrictions determined by the nasal anatomy, which together result in low therapeutic concentrations at the CNS $[50,51]$.

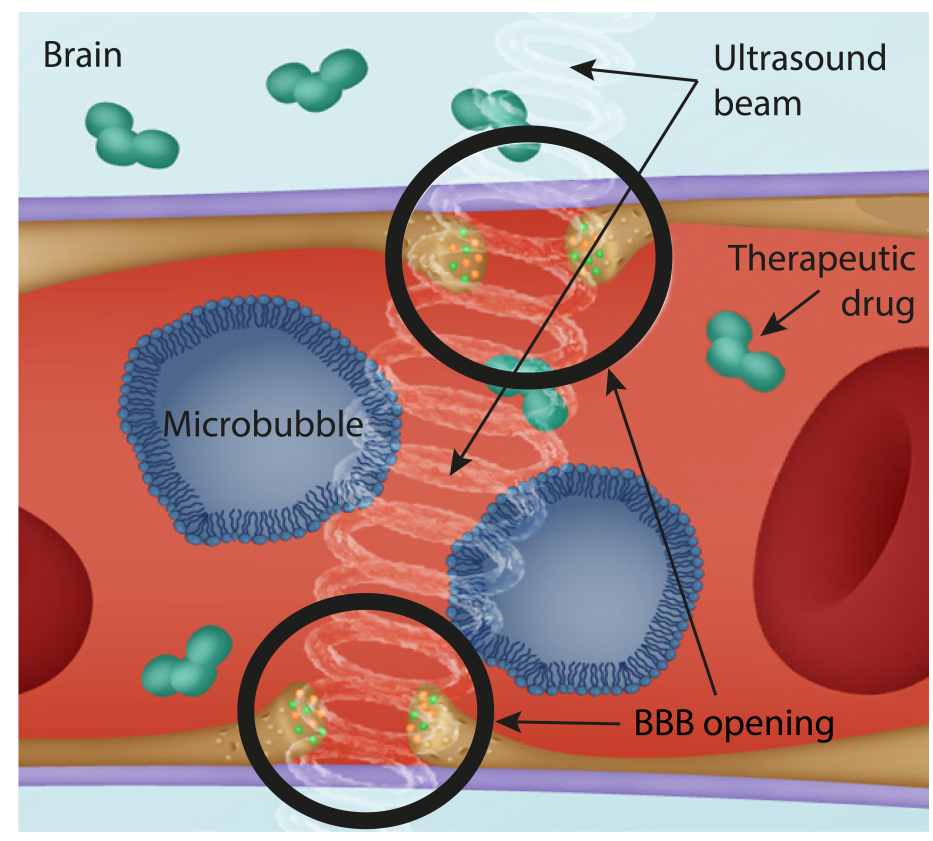

Figure 1.2: Non-invasive, local, transient and safe BBB opening by using microbubble-enhanced FUS. Therapeutic drugs engineered to treat brain-related diseases can easily pass through the BBB. Modified from [34]. 
Aside from the previous non-invasive approaches, which show poor localization of the delivered drug, there is one which presents the best trade-off between drug distribution/localization effectiveness and safeness for the CNS. The most powerful and used among all non-invasive approaches, proved pre- and clinically in treatments for several brain diseases and showing great potential is microbubble-enhanced focused ultrasound [52], which combines the use of focused ultrasound (FUS) beams with the injection of microbubbles into the bloodstream. This is a noninvasive method that uses the medium-intensity vibration of ultrasound waves (mechanical waves) to excite the microbubbles circulating through the brain capillaries, to mechanically separate the tight junctions forming the BBB in a transient, local and safe manner, thus allowing the drug to cross the BBB (as sketched in Fig. 1.2). This technique allows the delivery of therapeutic molecules greater than 400 Da [38].

\subsubsection{Focused ultrasound as the promising approach for CNS drug delivery}

One implantable (invasive) and three extracranial (non-invasive) devices have been clinically tested to open the BBB (see Fig. 1.3). On the one hand, the Sonocloud- $1^{\circledR}$ and Sonocloud- $9^{\circledR}$ are implantable ultrasound devices developed by CarThera (Paris, France) [54, 55]. These devices are introduced inside the cranial cavity through a craniotomy right after a surgical procedure for a biopsy or a tumor resection [56]. The working frequency is $1.05 \mathrm{MHz}$. Large and superficial brain structures are good targets for implantable devices as it's an unfocused technology. However, small isolated structures can not be targeted locally. On the other hand, a single-element, extracranial and focused-ultrasound device guided using neuronavigation technology is being developed by Columbia University [57], working at $0.25 \mathrm{MHz}$ for $\mathrm{AD}$. For the treatment of CNS tumors there are other works using a single-element, extracranial, focused-ultrasound device showing feasibility in animal models [58-60]. An advantage compared to implantable devices is that superficial and deep brain structures can be locally targeted. The focal size is medium compared to implantable devices, but the advantage is that the energy is focused on the desired target and not on adjacent ones. However, this extracranial device has to deal with the aberration effects generated by the complex skull bone, leading to 
(a)
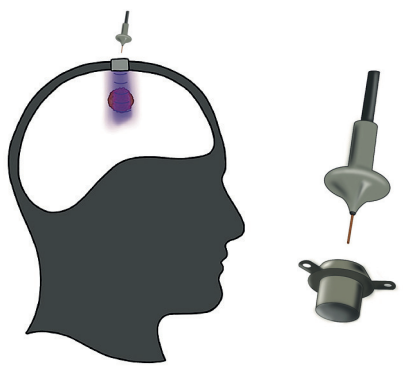

(c)

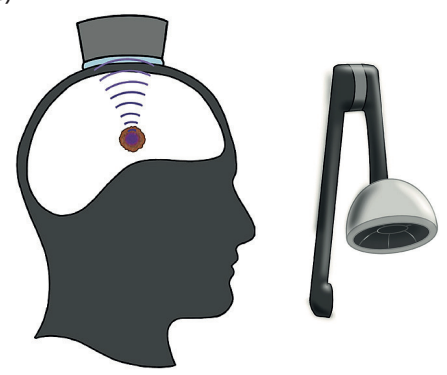

(b)
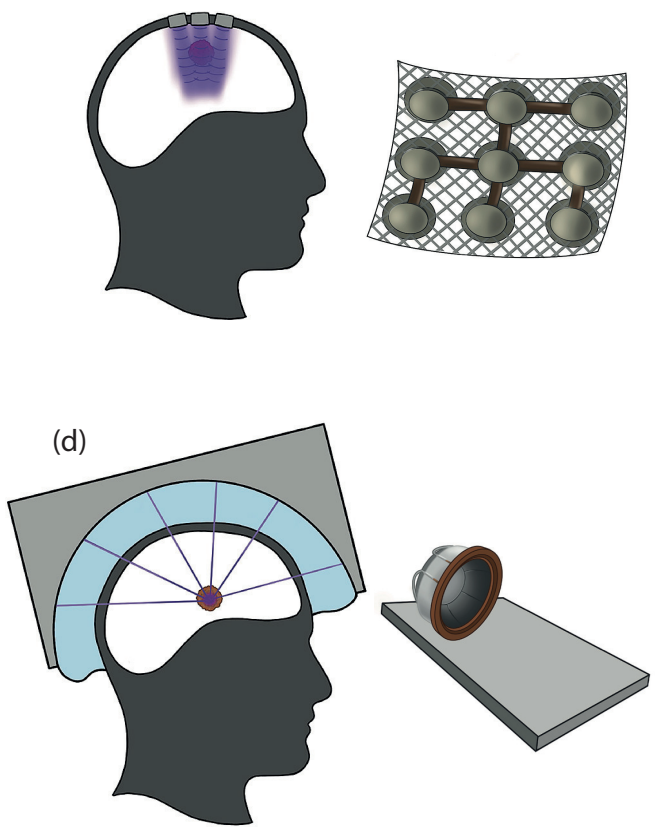

Figure 1.3: Ultrasound devices to disrupt the BBB in clinical procedures. (a) Implantable and unfocused single-element device (SonoCloud-1 ${ }^{\circledR}$ ). (b) Implantable and unfocused nine-element device (SonoCloud-9 ${ }^{\circledR}$ ). (c) Extracranial single-element focused device. (d) Extracranial hemispherical focused multi-element (ExAblate ${ }^{\circledR}$, NaviFUS ${ }^{\circledR}$ ). Modified from [53].

ultrasound distortions and attenuation. Furthermore, another extracranial and focused-ultrasound technology to locally target CNS structures is the multi-element device ExAblate ${ }^{\circledR}$ [61], developed by InSightec (InSightec, Tirat Carmel, Israel). It is a hemispherical ultrasound helmet containing 1024 transducers working at $220 \mathrm{kHz}$ guided using MRI. The skull aberrations can be corrected with this multi-element device. However, it is complicated to target wide volumes, e.g., a cancer brain tumor, as the focus size is reduced, and electronic steering of the focus has to be performed to cover large structures, in addition to the real-time 
MRI monitoring, leading to very complex, high- and time-cost clinical procedures. Another external, multichannel hemispherical phased-array ultrasound system is the NaviFUS ${ }^{\circledR}$ System [62], designed by NaviFUS (NaviFUS, Taiwan), which recently was used clinically in humans for CNS tumors [63].

\subsection{Thesis motivation}

Nowadays, treatments for neurodegenerative diseases such as Parkinson's Disease are still limited. On the one hand, existing technologies to open the $\mathrm{BBB}$ are not efficient enough to perform a fast and accurate procedure. On the other hand, existing therapeutic drugs still need to be further investigated to provide an effective treatment for the diseased brain tissue. Thereby, the first step to take is improving the technique to open the BBB.

Microbubble-enhanced FUS is the best current technique to achieve a local, transient, safe and highly non-invasive disruption of the BBB, thus allowing the treatment of brain diseases by enabling drugs to access the CNS. Several clinical trials have been FDA-approved based on microbubble-enhanced FUS. However, the treatment for neurological diseases, such as Alzheimer's or Parkinson's, remains limited because the complex ultrasound focusing required is not completely achieved by existing FUS technologies. For these treatments, the diseased brain structures have a complex and large-size morphology; however, both single-element and multi-element devices cannot provide an entire distribution of the ultrasound beam through the whole diseased brain structure. On the one hand, the single-element device would have to be displaced mechanically, which implies having an accurate control of the transducer with respect to the patient's head in each position, and as well several microbubble-injections would be required for the entire treatment. Another limitation is the aberration of the focus and beam attenuation that will vary during the scanning with the different incidence angles and the heterogeneity of the skull bone. On the other hand, the multi-element system allows to cover the whole brain structure by steering the beam electronically. However, this device provides a smaller focus size and, therefore, a higher number of sonications are required, which implies a constant MRI monitoring for each position of the focus, leading to an excessive increase in the cost of 
this treatment.

\subsubsection{Objectives}

The objective of this thesis is to develop a novel technology based on acoustic holograms with three principles: first, to provide a high-quality treatment by increasing the overlapped volume and at the same time reducing the clinical resources required; second, to be simple by using a single-element device which provides a fast pre-clinical planning and an easy treatment procedure; and third, to be low-cost so that it can be affordable to every clinical center all over the world, and therefore easily and widely used. The proposed strategy using acoustic holograms is ambitious in terms of its potential to overcome the limitations and lacks of existing BBB opening techniques based on using FUS and microbubbles, as well as other biomedical applications such as clot manipulation, trapping of kidney stone fragments, imaging, or guiding of drug-delivery carriers, where Bessel and vortex beams define a promising path. Thereby, using low-cost 3D-printed acoustic holographic lenses coupled to a single-element transducer, the ultrasonic beam can be accurately controlled compared to any of the conventional approaches.

This dissertation is structured in seven chapters. This first chapter provided an overview of brain diseases and their impact worldwide, taking special interest in developed treatments using FUS. From chapter 2 to 6 , it is demonstrated the feasibility and capability of the proposed novel technology, based on acoustic holograms, to considerably improve the current state-of-the-art FUS methods to treat brain diseases, through several biomedical applications: chapter 2 deals with the development of a technique based on acoustic holographic lenses which coupled to a single-element transducer allow the generation of acoustic holograms through the human skull; in chapter 3 it is experimentally demonstrated for the first time the feasibility and capability of acoustic holograms in mice in-vivo to produce a simultaneous bilateral BBB opening; chapter 4 is oriented towards another biomedical application such as chemotherapy, where acoustic holograms can increase the total treated volume by producing sharp acoustic images in the brain; chapter 5 turns around the use of acoustic holograms for the generation of acoustic vortices, which are beams useful for some biomedical applications such as particle trapping 
and manipulation, showing here the capability of accurate twisting a FUS beam through the human skull; in chapter 6 it is explored another application on acoustic holograms to reconstruct ideal Bessel beams of zeroth and higher-order, where the axial distribution is constant, that can be useful for some biomedical procedures. Finally, the last chapter contains a general discussion and conclusions of the development of this doctoral thesis.

\section{References of Chapter 1}

[1] A. M. Sousa, K. A. Meyer, G. Santpere, F. O. Gulden, and N. Sestan, "Evolution of the human nervous system function, structure, and development," Cell, vol. 170, no. 2, pp. 226-247, 2017.

[2] K. Pattabiraman, S. K. Muchnik, and N. Sestan, "The evolution of the human brain and disease susceptibility," Current Opinion in Genetics 85 Development, vol. 65, pp. 91-97, 2020.

[3] S. Herculano-Houzel, "The human advantage: How our brains became remarkable," 2016.

[4] C. E. Collins, E. C. Turner, E. K. Sawyer, J. L. Reed, N. A. Young, D. K. Flaherty, and J. H. Kaas, "Cortical cell and neuron density estimates in one chimpanzee hemisphere," Proceedings of the National Academy of Sciences, vol. 113 , no. 3, pp. 740-745, 2016.

[5] J. H. Lui, D. V. Hansen, and A. R. Kriegstein, "Development and evolution of the human neocortex," Cell, vol. 146, no. 1, pp. 18-36, 2011.

[6] J. C. Silbereis, S. Pochareddy, Y. Zhu, M. Li, and N. Sestan, "The cellular and molecular landscapes of the developing human central nervous system," Neuron, vol. 89, no. 2, pp. 248-268, 2016.

[7] E. D. Louis and P. L. Faust, "Essential tremor pathology: neurodegeneration and reorganization of neuronal connections," Nature Reviews Neurology, pp. $1-15,2020$.

[8] J. Benito-León, F. Bermejo-Pareja, J.-M. Morales, S. Vega, and J.-A. Molina, "Prevalence of essential tremor in three elderly populations of central spain," Movement disorders: official journal of the Movement Disorder Society, vol. 18, no. 4, pp. 389-394, 2003. 
[9] J. Benito-León and E. D. Louis, "Clinical update: diagnosis and treatment of essential tremor," The Lancet, vol. 369, no. 9568, pp. 1152-1154, 2007.

[10] E. D. Louis and J. J. Ferreira, "How common is the most common adult movement disorder? update on the worldwide prevalence of essential tremor," Movement Disorders, vol. 25, no. 5, pp. 534-541, 2010.

[11] E. D. Louis, "Essential tremor," The Lancet Neurology, vol. 4, no. 2, pp. 100$110,2005$.

[12] C. Vilariño-Güell, O. A. Ross, C. Wider, B. Jasinska-Myga, S. A. Cobb, A. I. Soto-Ortolaza, J. M. Kachergus, B. H. Keeling, J. C. Dachsel, H. L. Melrose, et al., "Lingo1 rs9652490 is associated with essential tremor and parkinson disease," Parkinsonism \& related disorders, vol. 16, no. 2, pp. 109-111, 2010.

[13] E. D. Louis, M. Gerbin, and M. Galecki, "Essential tremor 10, 20, 30, 40: clinical snapshots of the disease by decade of duration," European journal of neurology, vol. 20, no. 6, pp. 949-954, 2013.

[14] H. Stolze, G. Petersen, J. Raethjen, R. Wenzelburger, and G. Deuschl, "The gait disorder of advanced essential tremor," Brain, vol. 124, no. 11, pp. 22782286, 2001.

[15] V. Chandran, P. Pal, J. Reddy, K. Thennarasu, R. Yadav, and N. Shivashankar, "Non-motor features in essential tremor," Acta neurologica scandinavica, vol. 125, no. 5, pp. 332-337, 2012.

[16] E. D. Louis, "Non-motor symptoms in essential tremor: a review of the current data and state of the field," Parkinsonism 8 related disorders, vol. 22, pp. S115-S118, 2016.

[17] C. H. Halpern, V. Santini, N. Lipsman, A. M. Lozano, M. L. Schwartz, B. B. Shah, W. J. Elias, G. R. Cosgrove, M. T. Hayes, N. McDannold, et al., "Three-year follow-up of prospective trial of focused ultrasound thalamotomy for essential tremor," Neurology, vol. 93, no. 24, pp. e2284-e2293, 2019 .

[18] R. M. Jones, Y. Huang, Y. Meng, N. Scantlebury, M. L. Schwartz, N. Lipsman, and K. Hynynen, "Echo-focusing in transcranial focused ultrasound thalamotomy for essential tremor: A feasibility study," Movement Disorders, 2020. 
[19] J. A. Pineda-Pardo, D. Urso, R. Martínez-Fernández, R. Rodríguez-Rojas, M. Del-Alamo, P. Millar Vernetti, J. U. Máñez-Miró, F. HernándezFernández, E. de Luis-Pastor, L. Vela-Desojo, et al., "Transcranial magnetic resonance-guided focused ultrasound thalamotomy in essential tremor: a comprehensive lesion characterization," Neurosurgery, vol. 87, no. 2, pp. 256-265, 2020.

[20] W. Poewe, K. Seppi, C. M. Tanner, G. M. Halliday, P. Brundin, J. Volkmann, A.-E. Schrag, and A. E. Lang, "Parkinson disease," Nature reviews Disease primers, vol. 3, no. 1, pp. 1-21, 2017.

[21] M. C. De Rijk, W. A. Rocca, D. Anderson, M. Melcon, M. Breteler, and D. Maraganore, "A population perspective on diagnostic criteria for parkinson's disease," Neurology, vol. 48, no. 5, pp. 1277-1281, 1997.

[22] R. Balestrino and A. H. Schapira, "Parkinson disease," European journal of neurology, vol. 27, no. 1, pp. 27-42, 2020.

[23] R. J. Castellani, R. K. Rolston, and M. A. Smith, "Alzheimer disease," Disease-a-month: DM, vol. 56, no. 9, p. 484, 2010.

[24] M. Robinson, B. Y. Lee, and F. T. Hane, "Recent progress in alzheimer's disease research, part 2: genetics and epidemiology," Journal of Alzheimer's Disease, vol. 57, no. 2, pp. 317-330, 2017.

[25] J. M. Long and D. M. Holtzman, "Alzheimer disease: an update on pathobiology and treatment strategies," Cell, vol. 179, no. 2, pp. 312-339, 2019.

[26] C. Reitz, C. Brayne, and R. Mayeux, "Epidemiology of alzheimer disease," Nature Reviews Neurology, vol. 7, no. 3, pp. 137-152, 2011.

[27] R. Mayeux and Y. Stern, "Epidemiology of alzheimer disease," Cold Spring Harbor perspectives in medicine, vol. 2, no. 8, p. a006239, 2012.

[28] L. Mucke, "Alzheimer's disease," Nature, vol. 461, no. 7266, pp. 895-897, 2009 .

[29] D. B. Miller and J. P. O'Callaghan, "New horizons for focused ultrasound (fus)-therapeutic applications in neurodegenerative diseases," Metabolism, vol. 69, pp. S3-S7, 2017.

[30] N. Lipsman, Y. Meng, A. J. Bethune, Y. Huang, B. Lam, M. Masellis, N. Herrmann, C. Heyn, I. Aubert, A. Boutet, et al., "Blood-brain barrier opening in alzheimer's disease using mr-guided focused ultrasound," Nature communications, vol. 9, no. 1, pp. 1-8, 2018. 
[31] W. M. Pardridge, "Brain metabolism: a perspective from the blood-brain barrier," Physiological reviews, vol. 63, no. 4, pp. 1481-1535, 1983.

[32] P. Ehrlich, "Das sauerstoff-bedurfnis des organismus," Eine farbenanalytische studie, 1885.

[33] J. Kealy, C. Greene, and M. Campbell, "Blood-brain barrier regulation in psychiatric disorders," Neuroscience letters, vol. 726, p. 133664, 2020.

[34] R. Pandit, L. Chen, and J. Götz, "The blood-brain barrier: physiology and strategies for drug delivery," Advanced drug delivery reviews, vol. 165, pp. 114,2020 .

[35] J. Nolte, The human brain. Mosby/Elsevier,, 1993.

[36] N. J. Abbott, L. Rönnbäck, and E. Hansson, "Astrocyte-endothelial interactions at the blood-brain barrier," Nature reviews neuroscience, vol. 7, no. 1, p. $41,2006$.

[37] M. Bundgaard and N. J. Abbott, "All vertebrates started out with a glial blood-brain barrier 4-500 million years ago," Glia, vol. 56, no. 7, pp. 699$708,2008$.

[38] W. M. Pardridge, "The blood-brain barrier: bottleneck in brain drug development," NeuroRx, vol. 2, no. 1, pp. 3-14, 2005.

[39] W. M. Pardridge, "Drug targeting to the brain," Pharmaceutical research, vol. 24, no. 9, pp. 1733-1744, 2007.

[40] W. M. Pardridge, "Treatment of alzheimer's disease and blood-brain barrier drug delivery," Pharmaceuticals, vol. 13, no. 11, p. 394, 2020.

[41] W. M. Pardridge, Brain drug targeting: the future of brain drug development. Cambridge University Press, 2001.

[42] J. L. Cohen-Pfeffer, S. Gururangan, T. Lester, D. A. Lim, A. J. Shaywitz, M. Westphal, and I. Slavc, "Intracerebroventricular delivery as a safe, longterm route of drug administration," Pediatric neurology, vol. 67, pp. 23-35, 2017.

[43] S. D. Ferguson, K. Foster, and B. Yamini, "Convection-enhanced delivery for treatment of brain tumors," Expert review of anticancer therapy, vol. 7, no. sup1, pp. S79-S85, 2007. 
[44] L. Nam, C. Coll, L. Erthal, C. De la Torre, D. Serrano, R. Martínez-Máñez, M. Santos-Martínez, and E. Ruiz-Hernández, "Drug delivery nanosystems for the localized treatment of glioblastoma multiforme," Materials, vol. 11, no. 5 , p. $779,2018$.

[45] C.-T. Lu, Y.-Z. Zhao, H. L. Wong, J. Cai, L. Peng, and X.-Q. Tian, "Current approaches to enhance cns delivery of drugs across the brain barriers," International journal of nanomedicine, vol. 9, p. 2241, 2014.

[46] J. L. Mikitsh and A.-M. Chacko, "Pathways for small molecule delivery to the central nervous system across the blood-brain barrier," Perspectives in medicinal chemistry, vol. 6, pp. PMC-S13384, 2014.

[47] S. J. Gray, K. T. Woodard, and R. J. Samulski, "Viral vectors and delivery strategies for cns gene therapy," Therapeutic delivery, vol. 1, no. 4, pp. 517$534,2010$.

[48] M. Themis, S. N. Waddington, M. Schmidt, C. Von Kalle, Y. Wang, F. AlAllaf, L. G. Gregory, M. Nivsarkar, M. Themis, M. V. Holder, et al., "Oncogenesis following delivery of a nonprimate lentiviral gene therapy vector to fetal and neonatal mice," Molecular Therapy, vol. 12, no. 4, pp. 763-771, 2005 .

[49] L. R. Hanson and W. H. Frey, "Intranasal delivery bypasses the blood-brain barrier to target therapeutic agents to the central nervous system and treat neurodegenerative disease," BMC neuroscience, vol. 9, no. S3, p. S5, 2008.

[50] J. J. Lochhead and R. G. Thorne, "Intranasal delivery of biologics to the central nervous system," Advanced drug delivery reviews, vol. 64, no. 7, pp. 614$628,2012$.

[51] F. Erdő, L. A. Bors, D. Farkas, Á. Bajza, and S. Gizurarson, "Evaluation of intranasal delivery route of drug administration for brain targeting," Brain research bulletin, vol. 143, pp. 155-170, 2018.

[52] K. Hynynen, N. McDannold, N. Vykhodtseva, and F. A. Jolesz, "Noninvasive $\mathrm{mr}$ imaging-guided focal opening of the blood-brain barrier in rabbits," Radiology, vol. 220, no. 3, pp. 640-646, 2001.

[53] K. Beccaria, M. Canney, G. Bouchoux, C. Desseaux, J. Grill, A. B. Heimberger, and A. Carpentier, "Ultrasound-induced blood-brain barrier disruption for the treatment of gliomas and other primary cns tumors," Cancer Letters, 2020. 
[54] C. Horodyckid, M. Canney, A. Vignot, R. Boisgard, A. Drier, G. Huberfeld, C. François, A. Prigent, M. D. Santin, C. Adam, et al., "Safe long-term repeated disruption of the blood-brain barrier using an implantable ultrasound device: a multiparametric study in a primate model," Journal of neurosurgery, vol. 126, no. 4, pp. 1351-1361, 2017.

[55] A. Idbaih, M. Canney, L. Belin, C. Desseaux, A. Vignot, G. Bouchoux, N. Asquier, B. Law-Ye, D. Leclercq, A. Bissery, et al., "Safety and feasibility of repeated and transient blood-brain barrier disruption by pulsed ultrasound in patients with recurrent glioblastoma," Clinical Cancer Research, vol. 25, no. 13 , pp. 3793-3801, 2019.

[56] A. Carpentier, M. Canney, A. Vignot, V. Reina, K. Beccaria, C. Horodyckid, C. Karachi, D. Leclercq, C. Lafon, J.-Y. Chapelon, et al., "Clinical trial of blood-brain barrier disruption by pulsed ultrasound," Science translational medicine, vol. 8, no. 343, pp. 343re2-343re2, 2016.

[57] A. N. Pouliopoulos, S.-Y. Wu, M. T. Burgess, M. E. Karakatsani, H. A. Kamimura, and E. E. Konofagou, "A clinical system for non-invasive bloodbrain barrier opening using a neuronavigation-guided single-element focused ultrasound transducer," Ultrasound in medicine 85 biology, vol. 46, no. 1, pp. 73-89, 2020.

[58] H.-L. Liu, M.-Y. Hua, P.-Y. Chen, P.-C. Chu, C.-H. Pan, H.-W. Yang, C.-Y. Huang, J.-J. Wang, T.-C. Yen, and K.-C. Wei, "Blood-brain barrier disruption with focused ultrasound enhances delivery of chemotherapeutic drugs for glioblastoma treatment," Radiology, vol. 255, no. 2, pp. 415-425, 2010 .

[59] E.-J. Park, Y.-Z. Zhang, N. Vykhodtseva, and N. McDannold, "Ultrasoundmediated blood-brain/blood-tumor barrier disruption improves outcomes with trastuzumab in a breast cancer brain metastasis model," Journal of controlled release, vol. 163, no. 3, pp. 277-284, 2012.

[60] C.-Y. Ting, C.-H. Fan, H.-L. Liu, C.-Y. Huang, H.-Y. Hsieh, T.-C. Yen, K.C. Wei, and C.-K. Yeh, "Concurrent blood-brain barrier opening and local drug delivery using drug-carrying microbubbles and focused ultrasound for brain glioma treatment," Biomaterials, vol. 33, no. 2, pp. 704-712, 2012.

[61] T. Mainprize, N. Lipsman, Y. Huang, Y. Meng, A. Bethune, S. Ironside, C. Heyn, R. Alkins, M. Trudeau, A. Sahgal, et al., "Blood-brain barrier opening in primary brain tumors with non-invasive mr-guided focused ultrasound: a clinical safety and feasibility study," Scientific reports, vol. 9, no. 1, pp. 1-7, 2019. 
[62] K.-T. Chen, Y.-J. Lin, W.-Y. Chai, C.-J. Lin, P.-Y. Chen, C.-Y. Huang, J. S. Kuo, H.-L. Liu, and K.-C. Wei, "Neuronavigation-guided focused ultrasound (navifus) for transcranial blood-brain barrier opening in recurrent glioblastoma patients: clinical trial protocol," Annals of Translational Medicine, vol. 8, no. 11, 2020.

[63] K.-T. Chen, W.-Y. Chai, Y.-J. Lin, C.-J. Lin, P.-Y. Chen, H.-C. Tsai, C.-Y. Huang, J. S. Kuo, H.-L. Liu, and K.-C. Wei, "Neuronavigation-guided focused ultrasound for transcranial blood-brain barrier opening and immunostimulation in brain tumors," Science Advances, vol. 7, no. 6, p. eabd0772, 2021.

[64] Cover image for this chapter, creator name: ustas, stock.adobe.com. 



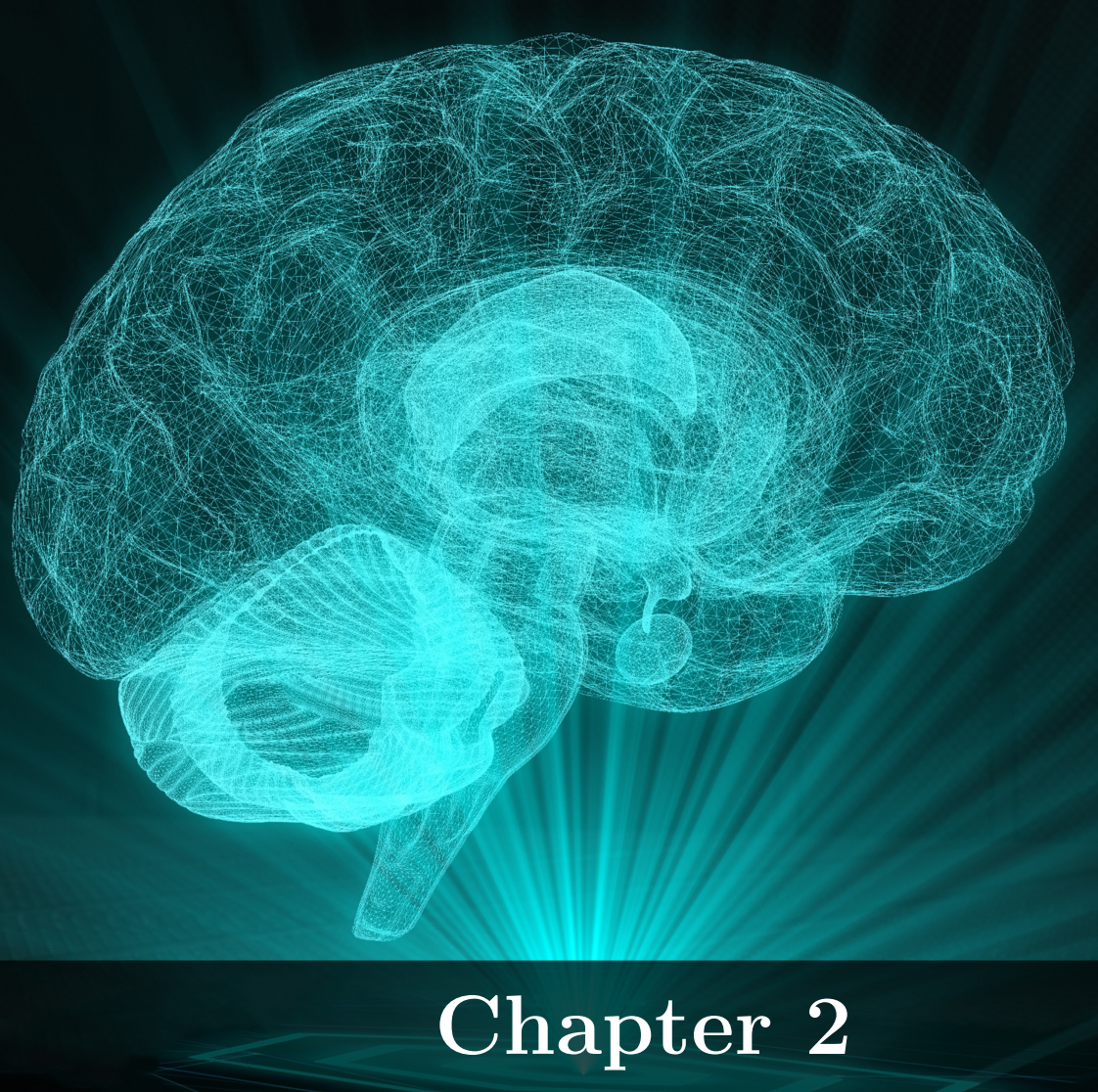

\section{Holograms to focus ultrasonic beams through the human skull}

Acoustic holograms are reported to overcome existing limitations in brain-related procedures. In this second chapter, low-cost 3D-printed acoustic holograms form acoustic images inside a human brain while correcting the skull aberrations. The shape of the FUS beams matches that of target CNS structures. First, the central points of both hippocampi were selected as targets, resulting an accurate simultaneous double focus. Second, a curved trajectory was selected as target, achieving a beam bending accordingly to the path. Finally, a slice of the right hippocampus was selected as a target volume, resulting a focus overlapping accurately with it. These results open alternative paths to spread incoming biomedical ultrasound applications, including BBB opening or neuromodulation. 


\subsection{Introduction}

Holography was invented by Dennis Gabor [1], a Hungarian-British electrical engineer and physicist who received the 1971 Nobel Prize in Physics. Holographic plates are surfaces that when illuminated by a wave, typically light, modify the phase of the transmitted or reflected wavefront in such a manner that a complex image can be formed [2-4]. In recent years, subwavelength thickness holographic metasurfaces have been designed using structured materials with subwavelength resonances, namely metamaterials $[5,6]$. Analogously, in acoustics, a broad range of locally-resonant structures have been proposed to obtain precise control of the wavefront at a subwavelength scale $[7,8]$, including effective negative mass density [9] and/or bulk modulus metamaterials [10, 11]. Acoustic metamaterials allow accurate control of the reflected [12-16] or transmitted wavefronts [17-19]. The use of these structures has been exploited to design negative-refraction superlenses [20] or hyperbolic dispersion-relation hyperlenses [21] that exhibit subwavelength focusing properties in the near field. Holographic lenses have also been reported in acoustics to generate complex acoustic fields [22-25]. Multifrequency holograms have also been reported [26]. Equivalently, using phased-array sources, the generation of complex beam patterns [27], self-bending and bottle beams [28], or vortex beams for particle levitation and manipulation has been reported [29]. Mixed approaches between metamaterials and phased arrays have also been presented [30].

In these applications, holographic lenses have demonstrated the ability to manipulate acoustic waves in free media, i.e., without inhomogeneities. However, when using ultrasound in biomedical-engineering applications, acoustic beams encounter multiple tissue layers of complex geometry with non-homogeneous properties in their path. Accurate control of the focused beam is at the basis of FUS therapy techniques, e.g., as in high-intensity FUS hyperthermia, thermal ablation or histotripsy, or in extracorporeal shockwave lithotripsy [31, 32]. Focusing directly into human soft tissues can efficiently be achieved using conventional systems as ultrasound beam aberrations are typically small in these media [33]. However, when the target tissue lays behind high-impedance tissues, e.g., soft tissue surrounded by bones, the beam experiences strong aberrations due to refraction, reflec- 
tion, and absorption processes [34]. Some applications make use of existing acoustic windows by targeting tissues from specific locations. However, in transcranial propagation, skull bones are always present in the path towards the CNS. In this way, the precise control of acoustic focus into the CNS is mainly limited due to the strong phase aberrations produced by the refraction and attenuation of the skull [35].

To overcome these limitations, minimally invasive techniques have been developed in the past to design active focusing systems using the time-reversal (TR) invariance of the acoustic propagation [36] or phase conjugation methods [37]. In minimally invasive techniques, a small acoustic source is introduced into the skull, together with a biopsy catheter. When the catheter reaches the target tissue, it radiates a short ultrasonic pulse that travels outwards, and it is recorded by a hemispherical multi-element array surrounding the patient's head. Then, the elements of the phased array are set to re-emit the time-reversed recorded waveforms (or phase-conjugated harmonic signals). Due to spatial reciprocity and time-reversal invariance of the acoustic media, the generated wavefront focuses at the catheter location, i.e., at target tissue [36]. Later, it was demonstrated that noninvasive versions of these techniques can be obtained using numerical simulations [38, 39]. In these techniques, a tomographic image is previously obtained from a patient's head to extract the geometry of the skull and its acoustic properties [39]. Using full-wave simulations, the time-reversed wavefront is calculated by exciting the simulation with a virtual source at the desired focal spot. Then, a physical hemispherical phased array is excited with the synthetic time-reversed waveforms [40] or phase-conjugated signals [38], and sharp focusing through skull-aberration layers is retrieved. Other techniques include the optimization of phase arrays using magnetic resonance imaging (MRI) to maximize acoustic-radiation-force-induced displacements into the target focus [41]. However, up-to-date phased-array systems are restricted to a limited number of channels, e.g., 1024 for the Exablate ${ }^{\circledR}$ Model 4000 (InSightec, Ltd) [42], that are insufficient to fully record the required holographic information to conform a complex beam pattern. In addition, high-intensity focused ultrasound (HIFU) systems have a limited steering capability away from the geometric focus [43], e.g., a valid treatment volume of $30 \times 30 \times 40 \mathrm{~mm}^{3}$. As each of the piezoelectric elements forming the 
phased-array has a large size, the separation distance between elements is much greater than half wavelength and, consequently, relevant amplitude grating lobes appear.

Only a few theoretical works have tackled the problem of beam focusing through aberrating layers using metamaterials [44] or phase plates $[45,46]$. In Ref. [44], a two-dimensional (2D) configuration was proposed theoretically using a metasurface based on membranes. Recently, the use of phase plates to generate simple, focused sources have been reported to avoid beam aberrations in transcranial propagation [45]. However, the technique was limited to focus the beam into a single focal spot at the near field of the source. Besides, in some nonthermal transcranial ultrasound applications such as blood-brain-barrier opening [47] or neuromodulation [48], the ultrasound beam might be set to fully cover a geometrically complex CNS structure rather than focusing over a small focal spot.

In this work, we propose the use of 3D-printed holographic phase plates to produce ultrasonic fields of arbitrary shape into the human brain. The holographic lenses designed in this work allow the reconstruction of complex diffraction-limited acoustic images, including the compensation of the aberrations produced by a skull phantom.

In particular, we theoretically, numerically, and experimentally demonstrate the generation of several holographic patterns of increasing complexity, all with direct practical application to biomedicine ultrasound: an arbitrary set of points, an arbitrary curved line, and an arbitrary volume. First, we provide the conditions to generate a simple holographic pattern, i.e., a set of diffraction-limited focal points, as sketched in Fig. 2.1 (b). In particular, we extend the use of holographic lenses to generate bifocal beams, matching both foci simultaneously to the location of the left and right human hippocampi. Second, we demonstrate that ultrasonic beams with a curved trajectory along the internal CNS tissues can also be produced, as shown in Fig. 2.1 (c). In this way, the acoustic beam can be bent following arbitrary paths producing a self-bending beam inside the CNS. Finally, we report the generation of a beam pattern that overlaps with the volume of a specific CNS structure, as shown in Fig. 2.1 (d); in particular, we target the right human hippocampus. 
(a) Holographic plate

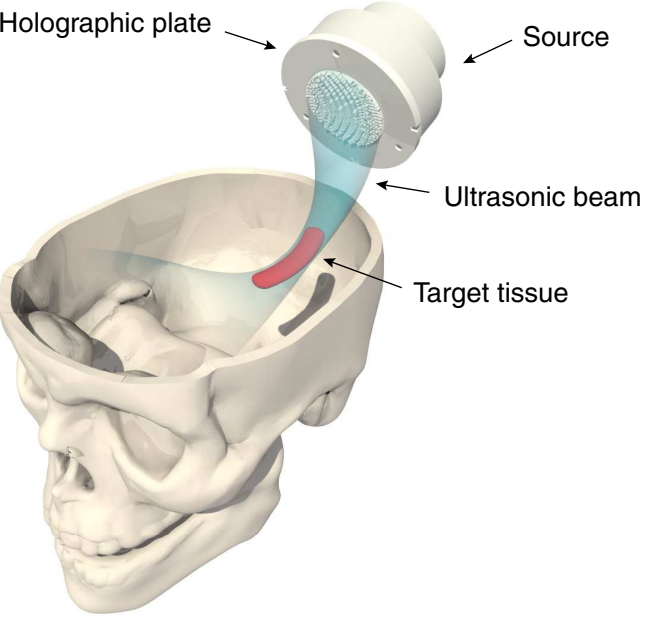

(b) Multiple point holographic focusing

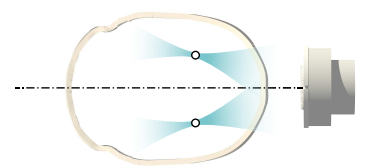

(c) Line holographic focusing

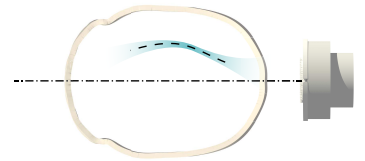

(d) Volumetric holographic focusing

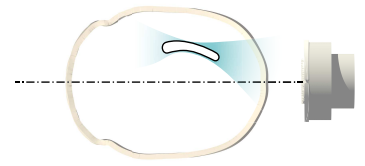

Figure 2.1: (a) Scheme of the holographic lens focusing over a target CNS structure. (b) Focusing on a set of arbitrary points (bifocal holographic lens). (c) Focusing over an arbitrary line (self-bending holographic lens). (d) Focusing over an arbitrary volume (volumetric holographic lens).

\section{$2.2 \quad$ Methods}

The process of hologram generation is composed of four steps. First, we extract the geometry and acoustic properties of a human skull from x-ray CT images, as shown in Fig. 2.2 (a), and then, we identify the target tissue structure, e.g., the right human hippocampus, from MRI tomographic images, as shown in Fig. 2.2 (b). Second, a back-propagation method is used to calculate the acoustic wavefront generated from a set of virtual sources and impinging on a holographic surface located outside the skull phantom, as shown in Fig. 2.2 (b). Third, the phase-plate lens is generated using the phase and amplitude of the recorded wavefront at the holographic surface, as shown in Fig. 2.2 (c). Finally, the lens is excited with a flat and uniform ultrasonic transducer, and the target acoustic image is reconstructed by either theoretical, numerical forward-propagation, or experimental methods, as shown in Fig. 2.2 (d). 
(a) $C T+M R l$ images

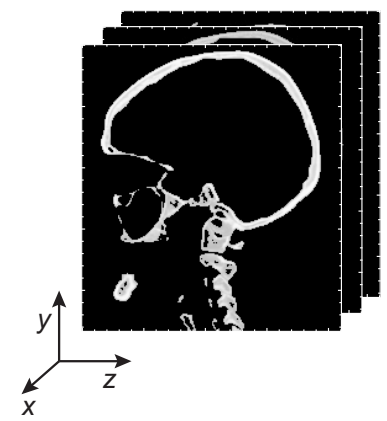

(b) Background propagation

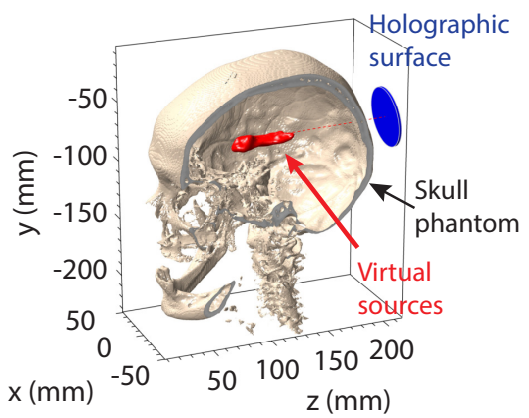

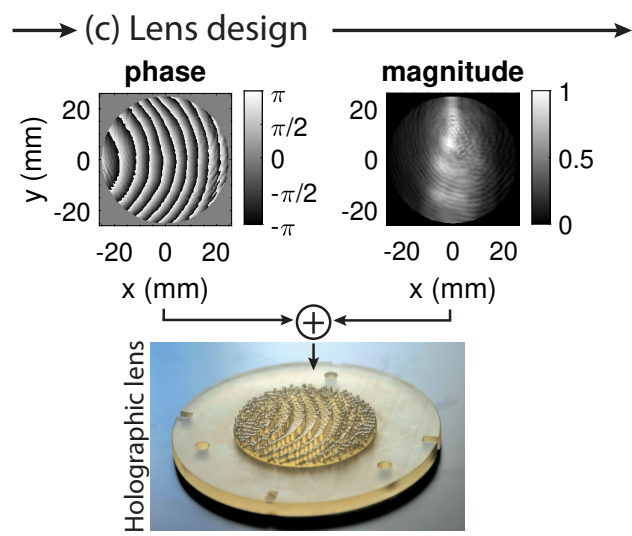

(d) Forward propagation

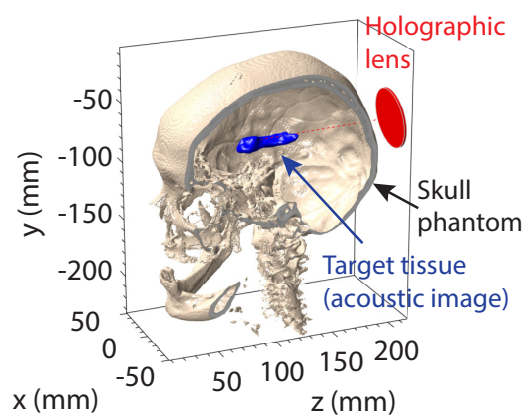

Figure 2.2: Hologram generation process. (a) CT+MRI tomographic images. (b) Selected target (red volume) acting as a virtual acoustic source and holographic recording surface (blue area). (c) Lens design using the TR back-propagated field. (d) Forward propagation from the holographic lens (red area) to the target tissue (blue volume).

\subsubsection{Tomographic image acquisition}

First, in order to model the skull geometry, we use the CT datasets of a female human head with an isotropic resolution of $1 \mathrm{~mm}$ (interpolated to $0.22 \mathrm{~mm}$ for the numerical simulation) from the National Library of 
Medicine's Visible Human Project available for general use by the University of Iowa. Experiments are conducted in a 3D-printed skull phantom, while, in addition, we include full-wave simulations using the acoustical properties of the skull bones. Thus, for the skull-phantom simulations, we use homogeneous acoustical parameters matching those of the 3D printing material. In contrast, for the realistic skull simulations, we use the same geometry but the inhomogeneous acoustical parameters of the skull are derived using the same CT data, converting the apparent density tomographic data in Hounsfield units to density and sound speed distributions using the linear-piecewise polynomials proposed in Refs. [49] and [50].

After, we use a human atlas made publicly available by the International Consortium for Brain Mapping (ICBM) from the Laboratory of Neuro Imaging [51]. This atlas provides us T1-weighted MRI data used to identify the shape and location of the human hippocampus. In particular, for segmentation, we use ITK-SNAP software [52] to obtain the shape and location of the left and right hippocampi.

\subsubsection{Calculation methods}

We use two methods of increasing complexity to estimate the back and forward acoustic fields: a semi-analytical method using the Rayleigh-Sommerfeld diffraction integral, and a pseudo-spectral time-domain simulation method.

On the one hand, for theoretical calculations in homogeneous media, i.e, in water without the skull phantom, the acoustic pressure field given by $p(\mathbf{r})$ at point $\mathbf{r}$, generated by a moving surface $S$ of arbitrary shape located at coordinates $\mathbf{r}_{0}$ and vibrating with a complex particle velocity $v_{0}\left(\mathbf{r}_{0}\right)$ normal to the surface, is given by the Rayleigh-Sommerfeld diffraction integral [53]:

$$
p(\mathbf{r}, \omega)=\frac{i \omega \rho_{0}}{2 \pi} \int_{S} \frac{v_{0}\left(\mathbf{r}_{0}\right) \exp \left(-i k_{0}\left|\mathbf{r}-\mathbf{r}_{0}\right|\right)}{\left|\mathbf{r}-\mathbf{r}_{0}\right|} d S,
$$

where $\omega=2 \pi f ; k_{0}=\omega / c_{0}, c_{0}$ and $\rho_{0}$ are the wavenumber, sound speed and density of water. Note that in Eq. (2.1), diffraction is captured exactly as compared with angular spectrum methods [54], so it can be applied to high-aperture sources. 
On the other hand, for calculations including aberration layers, we use a pseudospectral simulation method with $k$-space dispersion correction to numerically integrate the linearized constitutive relations of acoustics [55, 56]. In an inhomogeneous and absorbing media, the governing equations, i.e., the continuity equation, the momentum conservation equation, and the pressure-density relation, can be written as three-coupled first-order partial differential equations as

$$
\begin{aligned}
\frac{\partial \rho}{\partial t} & =-\rho_{0} \nabla \cdot \mathbf{u}-\mathbf{u} \cdot \nabla \rho_{0}, \\
\frac{\partial \mathbf{u}}{\partial t} & =-\frac{1}{\rho_{0}} \nabla p \\
p & =c_{0}^{2}\left(\rho+\mathbf{d} \cdot \nabla \rho_{0}-\mathrm{L} \rho\right),
\end{aligned}
$$

where $\mathbf{u}$ is the acoustic particle velocity, $\mathbf{d}$ is the acoustic particle displacement, $p$ is the acoustic pressure, $\rho$ is the acoustic density, $\rho_{0}$ is the ambient (or equilibrium) density, $c_{0}$ is the sound speed, and $L$ is a linear operator introducing the frequency-dependent absorption and dispersion [55]. Tissue absorption following a power law on frequency given by $\alpha(\omega)=\alpha_{0} \omega^{\gamma}$, where $\alpha_{0}$ is the absorption coefficient and $\gamma$ is the exponent of the frequency power law, together with its corresponding physical dispersion are included by the integro-differential operator as

$$
\mathrm{L}=\tau \frac{\partial}{\partial t}\left(-\nabla^{2}\right)^{(\gamma / 2)-1}+\eta\left(-\nabla^{2}\right)^{[(\gamma+1) / 2]-1}
$$

where $\tau=-2 \alpha_{0} c_{0}^{\gamma-1}$ and $\eta=2 \alpha_{0} c_{0}^{\gamma} \tan (\pi \gamma / 2)$ are the absorption and dispersion proportionality coefficients. This operator is solved efficiently using the fractional Laplacian in the $k$ space. This simulation method is implemented in the $k$-Wave toolbox for Matlab. It is selected as it provides low numerical dispersion compared with finite-differences methods [57]. We use a numerical grid with a spatial step of $\Delta x=\Delta y=$ $\Delta z=223 \mu \mathrm{m}$ and a numerical temporal step of $\Delta t=19.1 \mathrm{~ns}$, leading to a Courant-Friedrichs-Lewy number [55] of 0.13 in water and a spatial sampling of six grid points per wavelength in water for a frequency of $1.112 \mathrm{MHz}$. These parameters are fixed to all simulations in this chapter. 


\subsubsection{Lens design}

Under the assumption of reciprocity, time invariance, and linearity of the system, a time-reversal technique together with a direct method is used to design the phase-only holographic lens. First, we set some virtual sources inside the skull phantom, and the back-propagated field is estimated at a given surface outside the skull phantom. For the bifocal lens, two virtual sources are set as monopoles with the same phase and amplitude, located at the center of mass of the two hippocampi (right and left), as sketched in Fig. 2.1 (b). For the self-bending beam, a set of 50 virtual sources are located following an arbitrary curve as sketched in Fig. 2.1 (c), each source compensated by a phase factor of $\exp \left(i k_{z} z\right)$ accounting for the direction of arrival of the wavefront. Finally, for the volumetric hologram, as sketched in Fig. 2.1 (d), a set of virtual sources are spatially distributed with a separation of $\lambda / 6$ (to match the numerical grid used) over a sagittal plane of the right human hippocampus. The recorded field is captured at a given surface, i.e., at a holographic surface, outside the skull phantom.

Second, the recorded conjugated pressure distribution at the working frequency is used to design the physical lens. The lens surface is divided into squared pixels of different height, $h(x, y)$ and uniform width, $\Delta h$, as shown in Fig. 2.3. We assume each elastic column to vibrate longitudinally as a Fabry-Perot resonator. For each column, the field at the holographic plane located at $\mathbf{x}_{\mathbf{0}}=(x, y, d)$ is given by the complex transmission coefficient [58]:

$$
T\left(\mathbf{x}_{\mathbf{0}}\right)=\frac{2 Z \mathrm{e}^{-i k_{0}\left[d-h\left(\mathbf{x}_{\mathbf{0}}\right)\right]}}{2 Z \cos \left[k_{L} h\left(\mathbf{x}_{\mathbf{0}}\right)\right]+i\left(Z^{2}+1\right) \sin \left[k_{L} h\left(\mathbf{x}_{\mathbf{0}}\right)\right]},
$$

where $d$ is the distance from the bottom of the lens $(z=0 \mathrm{~mm})$ to the holographic surface, the normalized impedance is given by $Z=Z_{L} / Z_{0}$, and $Z_{0}=\rho_{0} c_{0}$ is the characteristic impedance of water and $Z_{L}=\rho_{L} c_{L}$, $k_{L}=\omega / c_{L}, \rho_{L}$ and $c_{L}$, are the impedance, wavenumber, density, and sound speed of the lens material. In order to obtain the height of each pixel of the lens, an analytic inversion of Eq. (2.6) is not possible due to the trigonometric terms. Instead, we first numerically evaluate the expression for a broad range of pixel heights ranging from a minimum height that is set to $h_{\min }=5 \mathrm{~mm}$ to guarantee structural consistency, to a given height that provides a phase of the transmission coefficient $2 \pi$ greater than for 


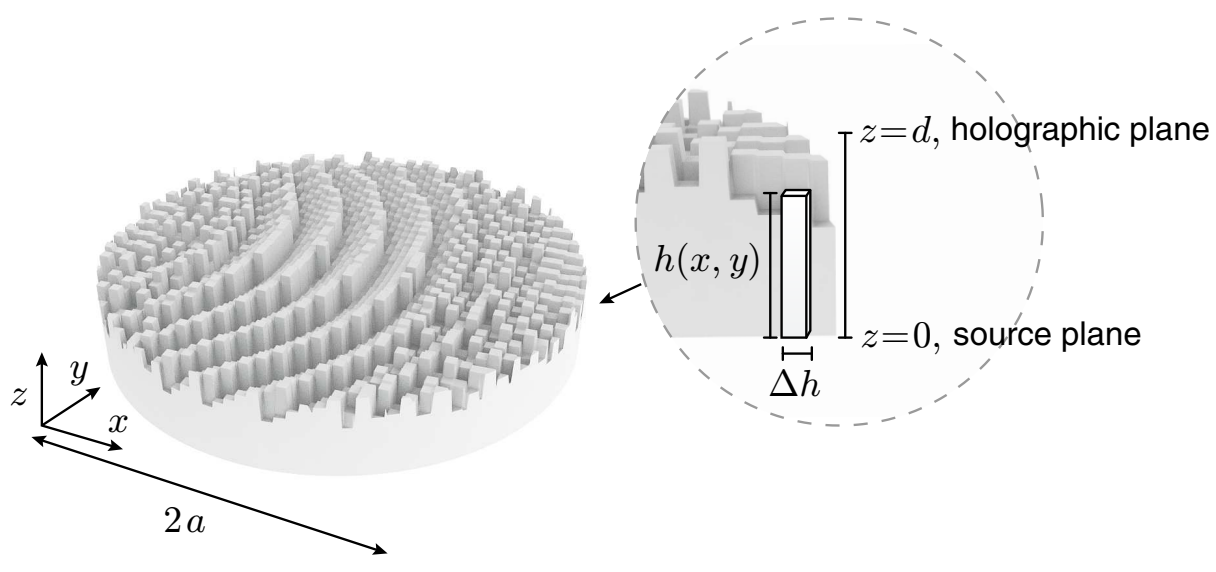

Figure 2.3: Geometry of the holographic lens. The lens, of aperture $2 a$, is subdivided in pixels of height $h(x, y)$. The source is located at $z=0 \mathrm{~mm}$, while the holographic plane is located at $z=d$.

$h_{\min }$, i.e., $d=15 \mathrm{~mm}$, and using steps of $1 \mu \mathrm{m}$, well below the printer accuracy. Finally, we perform interpolation using a cubic-spline method to obtain the height of the pixel as a function of the required phase. In this way, by tuning the height of each Fabry-Perot resonator, the phase at the output of each pixel can be tailored to that of a target holographic surface.

A detailed derivation of this expression can be found in Ref. [58]. The transmission coefficient for two materials as a function of the pixel height is given in Fig. 2.4 (a,b). Perfect agreement is observed between the transmission coefficient obtained using Eq. (2.6) and calculations using the analytical Transfer Matrix Method (TMM), see, e.g., Ref. [59, Chapter 11]. It can be observed that while both transmitted amplitude and phase depend on pixel height, the magnitude variations are relatively small due to the low contrast between the acoustic impedances of the lens and the surrounding water. For the PLA material, the magnitude of the transmission coefficient ranges between $|T|=[1,0.95]$ while for the material used in stereolithography (SLA) 3D printing, the transmission ranges between $|T|=[1,0.84]$.

However, using this kind of lens, the degree of freedom to modify the 

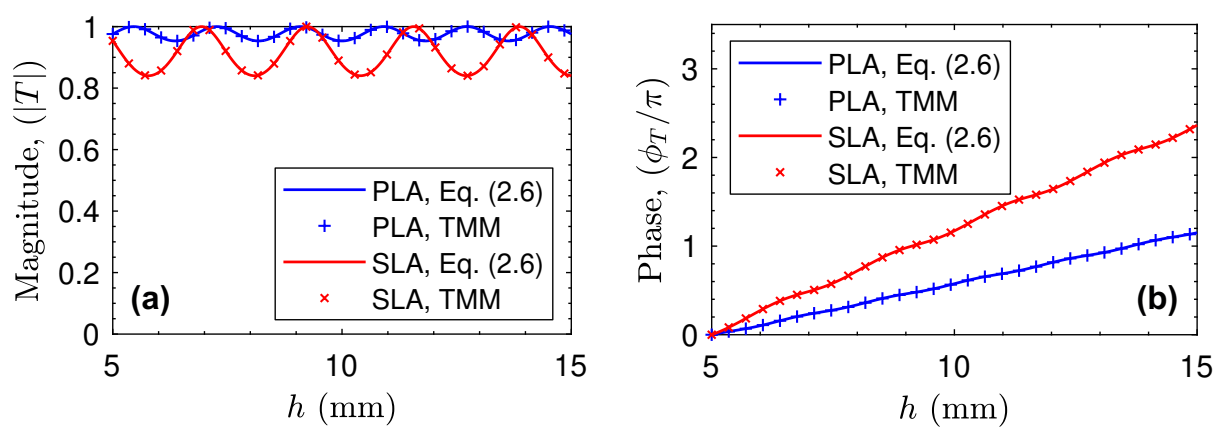

Figure 2.4: (a) Transmitted magnitude and (b) phase as a function of the pixel length for PLA material (blue) and SLA printing material (red). Continuous lines were calculated using Eq. (2.6) and Transfer Matrix Method (TMM) was used to calculate dotted lines.

magnitude of the field at the holographic surface is limited. Iterative methods have been employed in the past to obtain equivalent lenses only with phase distributions [22]. In this work, iterative methods are prohibitive: the 3D simulations including aberration layers involve long calculation times, e.g., $20 \mathrm{~h}$ in an Intel ${ }^{\circledR}$ Xeon ${ }^{\circledR}$ CPU E5-2680 v2 $2.80 \mathrm{GHz}, 256 \mathrm{~GB}$ RAM, using a CPU parallel implementation of the code. Instead, we use a direct method to estimate an equivalent holographic lens of uniform field magnitude [60]. The basis of this direct method is the sequential scanning of the pixels to modify the complex transmission coefficient. The method works as follows: first, the odd and even rows are scanned from opposite directions, and a bidirectional error of the diffusion process is calculated. The magnitude of each visited pixel is forced to be a constant value while the exact phase value is preserved. The resulting error is diffused to the neighboring pixels. Finally, the result gives a surface with a modified phase depending on the bidirectional error-diffusion process [60]. The main limitation of this method is that if the pixel width is small, it appears in areas with isolated long pixels, i.e., columns, that can experience bending modes. Note that this does not imply that a lens cannot be designed, but the theory we present here only applies to longitudinal modes on each pixel. The size of the pixels used in this work, 5/6 times the wavelength, is thick enough to ensure that the resonance frequency of 
the first bending mode is far away from the first longitudinal Fabry-Perot resonance frequency.

Third, lenses with an aperture of $2 a=50 \mathrm{~mm}$ are manufactured using 3D printing techniques. On the one hand, the bifocal holographic lens is manufactured by additive 3D printing techniques using Ultimaker 3 Extended (Ultimaker B.V., The Netherlands) with a resolution of $100 \mu \mathrm{m}$ in both lateral and axial directions and PLA material [see Fig. 2.5(a,b)]. As, in general, the height profile of the lens is smooth, we set the square pixel resolution to $\Delta h=0.22 \mathrm{~mm}$. The acoustical properties of polylactic acid material are obtained experimentally using a pulse-echo technique in a test cylinder, resulting in a measured sound speed of $c_{L}=1818 \mathrm{~m} / \mathrm{s}$, and a density of $\rho_{L}=1127 \mathrm{~kg} / \mathrm{m}^{3}$, matching with those reported in the existing literature [61], and the absorption is set to $\alpha=13.72 \mathrm{~dB} / \mathrm{cm}$
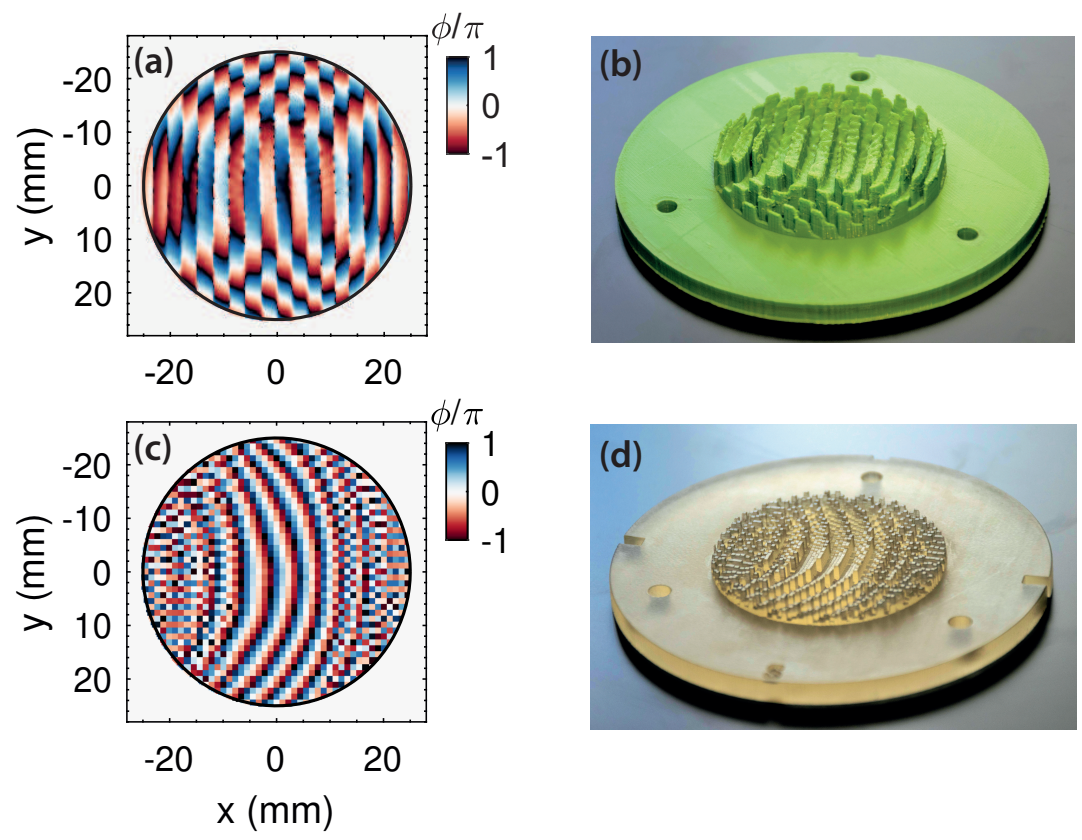

Figure 2.5: Back-propagated phase over the holographic surface for (a) multiple-point hologram and (c) volumetric hologram. (b,d) Photographs of the corresponding manufactured holographic lens. 
at $1.112 \mathrm{MHz}$ [61]. On the other hand, the self-bending and volumetric holographic lenses, which needed a more accurate printing technique for their complex pattern, are 3D printed using Polyjet techniques with an Objet30 printer (Stratasys, USA), with a resolution of $100 \mu \mathrm{m}$ and $28 \mu \mathrm{m}$ in lateral and axial directions, respectively, and using a photoresistive polymer (Veroclear ${ }^{\circledR}$, Stratasys, USA). As a result of the direct method to obtain the equivalent holographic lens of uniform field magnitude [60], the height distribution presents high spatial modulations [see Fig. 2.5 (c,d)]. Thus, the pixel resolution is increased to $\Delta h=1 \mathrm{~mm}$ to ensure each column vibrates as a longitudinal Fabry-Perot resonator avoiding bending modes around the working frequency for the volumetric hologram. For this material we experimentally estimate $c_{L}=2312 \mathrm{~m} / \mathrm{s}$ and $\rho_{L}=1191 \mathrm{~kg} / \mathrm{m}^{3}$ and $\alpha=3.06 \mathrm{~dB} / \mathrm{cm}$ at $1.112 \mathrm{MHz}$, matching the values reported in the existing literature [22].

\subsubsection{Skull phantom}

The geometry of the skull phantom is extracted from the 3D CT images as described previously. The sound speed and density distributions are first estimated from the apparent density given by the CT images in Hounsfield units $[49,50]$. Then, as the $3 \mathrm{D}$ printing technique results in homogeneous material, the acoustic properties, including the absorption are considered uniform along the skullbone volume [62-64]. The skull phantom is manufactured by additive 3D printing techniques using Ultimaker 3 Extended (Ultimaker B.V., The Netherlands) with a resolution of $100 \mu \mathrm{m}$ in both lateral and axial directions and using PLA material. The acoustic parameters for the 3D-printed phantom are the same as for the PLA lenses. Finally, for the simulations using a realistic skull, the acoustical parameters are derived using the CT data, converting the apparent density in Hounsfield units to density and sound-speed distributions using the linear-piecewise polynomials proposed in Refs. [49] and [50]. The density data ranges between $\rho_{0}=1000 \mathrm{~kg} / \mathrm{m}^{3}$ (water) and $\rho_{\max }=2206 \mathrm{~kg} / \mathrm{m}^{3}$ (bone), the sound-speed values range between $c_{0}=1500 \mathrm{~m} / \mathrm{s}$ and $c_{\max }=3117 \mathrm{~m} / \mathrm{s}$, matching those reported in the literature [32, 65] and the bone absorption is set to $12.6 \mathrm{~dB} / \mathrm{cm}$ at $1.112 \mathrm{MHz}[66]$. 


\subsubsection{Measurement setup}

The experiments are conducted inside a $1 \times 0.75 \times 0.5 \mathrm{~m}^{3}$ water tank filled with degassed and distilled water at $26^{\circ} \mathrm{C}$. The ultrasonic transducer is composed of a single element circular piezoceramic crystal (PZT26, Ferroperm Piezoceramics, Denmark) mounted in a custom designed stainless-steel housing with aperture $2 a=50 \mathrm{~mm}$ as shown in Fig. 2.6. The transducer is driven with a 50-cycle sinusoidal pulse burst at a frequency of $f=1.112 \mathrm{MHz}$ by a signal generator (14 bits, $100 \mathrm{MS} / \mathrm{s}$, model PXI5412, National Instruments) and amplified by a linear RF amplifier (ENI 1040L, $400 \mathrm{~W}, 55 \mathrm{~dB}$, ENI, Rochester, NY). First, vaseline is spread on the back of the lens, and afterwards, the lens is screwed onto the transducer to fix it. The 3D-printed skull phantom is accurately positioned using a 3D-printed holder, specially designed from the simulation data to hold the skull right in the same position as in simulation. The pressure field is measured

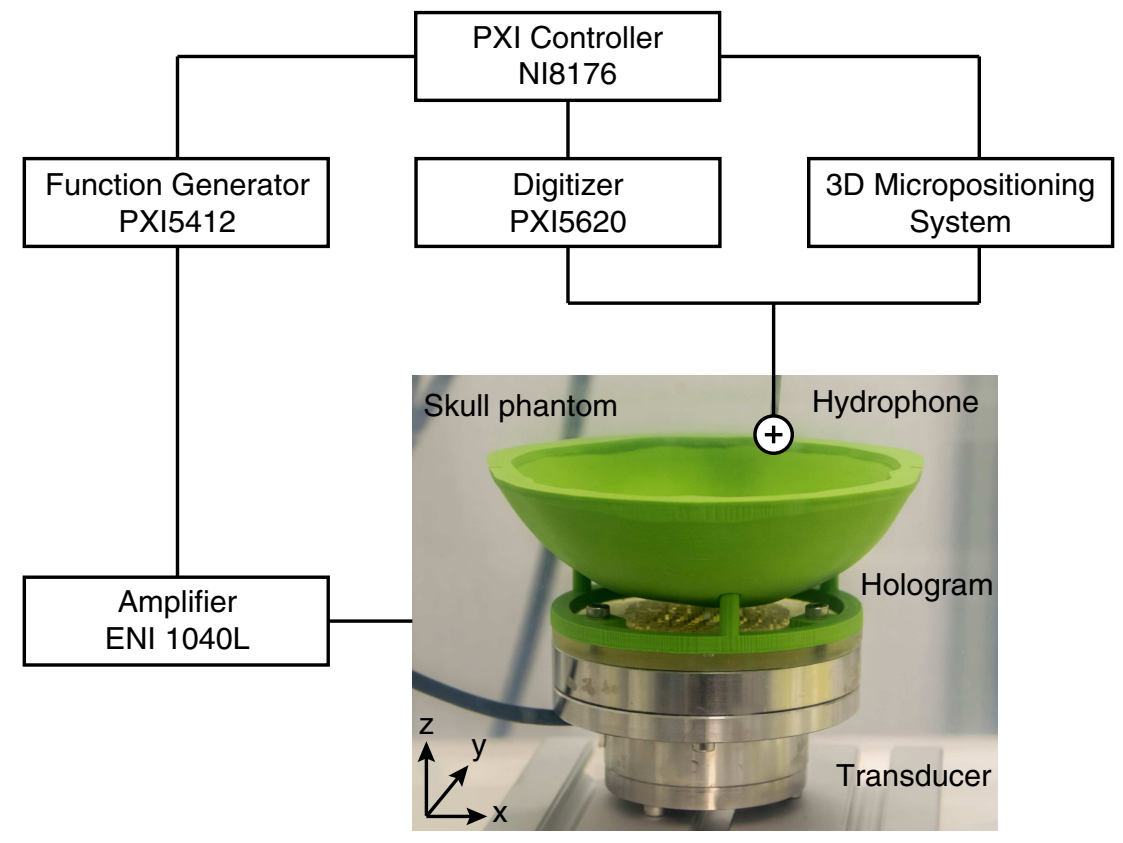

Figure 2.6: Experimental setup showing the block diagram and skull phantom inside the water tank. 
by a needle hydrophone with a $500 \mu \mathrm{m}$ active diameter $(149.6 \mathrm{mV} / \mathrm{MPa}$ sensitivity at $1.112 \mathrm{MHz}$, Model HNR-500, Onda) calibrated from $1 \mathrm{MHz}$ to $20 \mathrm{MHz}$. The source amplitude is set low enough to avoid any nonlinear effects in the propagation, we measure $1.8 \mathrm{kPa}$ at the focus for the bifocal lens. The hydrophone signals are digitized at a sampling rate of $64 \mathrm{MHz}$ by a digitizer (model PXI5620, National Instruments) averaged 100 times to increase the signal-to-noise ratio.

A 3D micropositioning system (OWIS GmbH) is used to move the hydrophone in three orthogonal directions with an accuracy of $10 \mu \mathrm{m}$. For the bifocal lens experiment, scanning an area for the sagittal cross-sections, $p(x, z)$, covers from -40 to $40 \mathrm{~mm}$ in the $x$-direction and 82 to $143 \mathrm{~mm}$ in the $z$-direction, using a step of $0.5 \mathrm{~mm}$ in both directions; for the transversal cross-section planes, $p(x, y)$, covers from -40 to $40 \mathrm{~mm}$ in the $x$-direction and -10 to $10 \mathrm{~mm}$ in the $y$-direction, using a step of $0.3 \mathrm{~mm}$. For the self-bending lens, the scanned area covers from 20 to $50 \mathrm{~mm}$ in the $z$-direction, -5 to $5 \mathrm{~mm}$ in the $x$-direction, and -5 to $5 \mathrm{~mm}$ in the $y$-direction, using the same spatial steps. Finally, for the volumetric holograms, the scanned area covers from 65 to $135 \mathrm{~mm}$ in the $z$-direction, -45 to $-5 \mathrm{~mm}$ in the $x$-direction, and -10 to $10 \mathrm{~mm}$ in the $y$-direction, using the same spatial steps. All the signal generation and acquisition processes are based on a NI8176 National Instruments PXI-Technology controller, which also controlled the micropositioning system. Temperature measurements are performed throughout the whole process to ensure temperature changes below $0.5^{\circ} \mathrm{C}$.

\subsection{Multiple-point holograms}

We start with the bifocal holographic lens. First, two points located at the center of mass of both left and right human hippocampi are selected. Second, we set this pair of points as the location of virtual sources for the TR method. For the lens designs in free media, i.e., without the skull, we use the Rayleigh-Sommerfeld diffraction integral (see Sec. 2.2 for further details). For the lens designs of holographic surfaces, including the skull-aberration layers, we use low-numerical-dispersion simulations based on pseudospectral methods [55]. In this way, the simultaneous back propagation of the fields irradiated by both virtual monopoles can be 
calculated at the holographic surface, which is located at the rear part of the skull. The phase-plate lens is designed using the conjugated complex field recorded at the surface. Then, the lens is placed at the location of the holographic surface, as shown in Fig. 2.7 (a), and a forward-propagation calculation is carried out to test the quality of the reconstructed acoustic image.

The field produced by the bifocal lens propagating through a human occipital-parietal skull phantom, including the compensation for the aberrations of the skull is shown in Figs. 2.7 (a-f). First, Figs. 2.7 (a) and 2.7 (b) show the axial-field cross-section, $p(x, y=0, z)$, using the pseudospectral simulation method and measured experimentally, respectively. We observe that the reconstructed field accurately matches the target foci, and the experimental results agree with the simulations. The corresponding transverse-field distributions at $z=105 \mathrm{~mm}$ are shown in Figs. 2.7 (c) and $2.7(\mathrm{~d})$, where sharp focusing is observed. The focal spots present
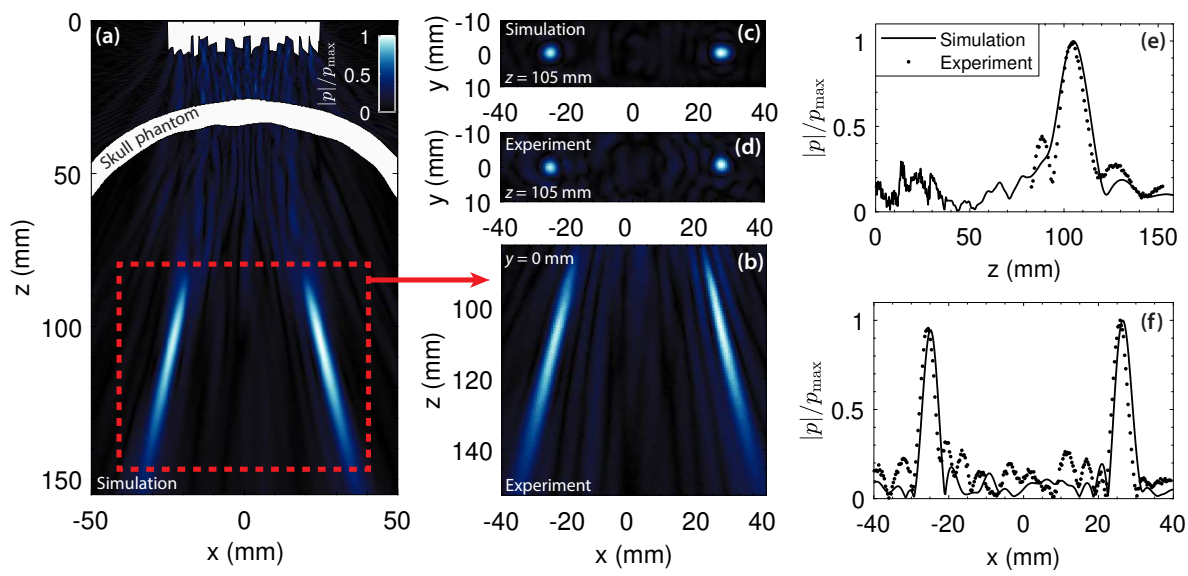

Figure 2.7: Axial-field cross-section obtained for the bifocal lens using simulations (a) and experiments (b). (c),(d) Corresponding transversal pressure-field distributions. Color bar in units normalized to the peak pressure. (e),(f) Simulated and experimental normalized axial and transversal field cross-sections, respectively. 
larger dimensions in the axial $(z)$ direction than in transverse ones, as expected from limited-aperture holographic lenses, where the spatial-spectral components in axial direction are limited by the finite-aperture source [67]. Axial (measured at $x=25 \mathrm{~mm}$ and $y=0 \mathrm{~mm}$ ) and transversal (measured at $z=105 \mathrm{~mm}$ and $y=0 \mathrm{~mm}$ ) cross-sections are shown in Figs. 2.7 (e) and 2.7 (f), respectively. Excellent agreement is observed between simulation and experiment for the axial-field profile at the focal region. A small secondary lobe located before the main one appears experimentally. The transverse profile shows a small lateral shift of $\pm 0.5 \mathrm{~mm}$ in both experimental foci towards the $x$-axis origin.

Note that, due to diffraction, the geometrical focus of a geometrically focused source does not correspond to the acoustic focus of the source [67]. In our case, the target location is set to $z=105.5 \mathrm{~mm}$, the acoustical focus of an equivalent focused source of the same frequency and aperture in water peaks at $z=99.8 \mathrm{~mm}$ and the focus of the lens peaks at $z=$ $100.4 \mathrm{~mm}$ and $z=100.1 \mathrm{~mm}$ in simulations and experiments including the skull phantom, respectively. These shifts correspond to errors of 0.6 and $0.3 \%$, respectively, showing the accuracy of the focusing performance of the holographic lenses.

\subsection{Self-bending beams}

The previous results show that holographic phase plates can retain phase information of multiple foci. Using this idea, we can set more complex targets following the shape of functional structures found in the CNS. Here, we set the holographic target pattern to a beam following a curved trajectory as those reported in homogeneous media without aberrating layers using active sources or metamaterials $[18,28,68]$. As the aberration layers are present in the real application, known analytical methods to calculate the phase of the 3D trajectory are, in principle, not available [28]. Instead, we use a TR method: a set of virtual sources are placed along this trajectory, and their back-propagated field is calculated. We set a factor of $\left(z / z_{\max }\right) \exp \left(i z k_{z}\right)$ to compensate for the amplitude and phase of each source to set the main direction of propagation, being $k_{z}$ the axial component of the wavevector and $z_{\max }$ the distance to the farthest virtual source ( $45 \mathrm{~mm}$ in this example). A sketch of the target trajec- 
tory is shown in Fig. 2.8 (a). The axial and transversal cross-sections of the forward-propagated field in water are shown in Figs. $2.8(\mathrm{a}, \mathrm{b})$, respectively. We observe that using the TR method, the self-bending beams can be obtained, and the beam accurately follows the target trajectory. A similar result is obtained using simulation and a lens made of elastic material, as shown in Figs. $2.8(\mathrm{c}, \mathrm{d})$. The experimental tests show a similar pressure-field distribution in comparison with theory using the Rayleigh-Sommerfeld integral and simulations using pseudospectral methods. A lateral shift of the peak pressure location of $0.3 \mathrm{~mm}$ in the $x$-direction is observed at $z=30 \mathrm{~mm}$ and $y=0 \mathrm{~mm}$ in the experiments.

Finally, when the aberration layer of the skull phantom is included,
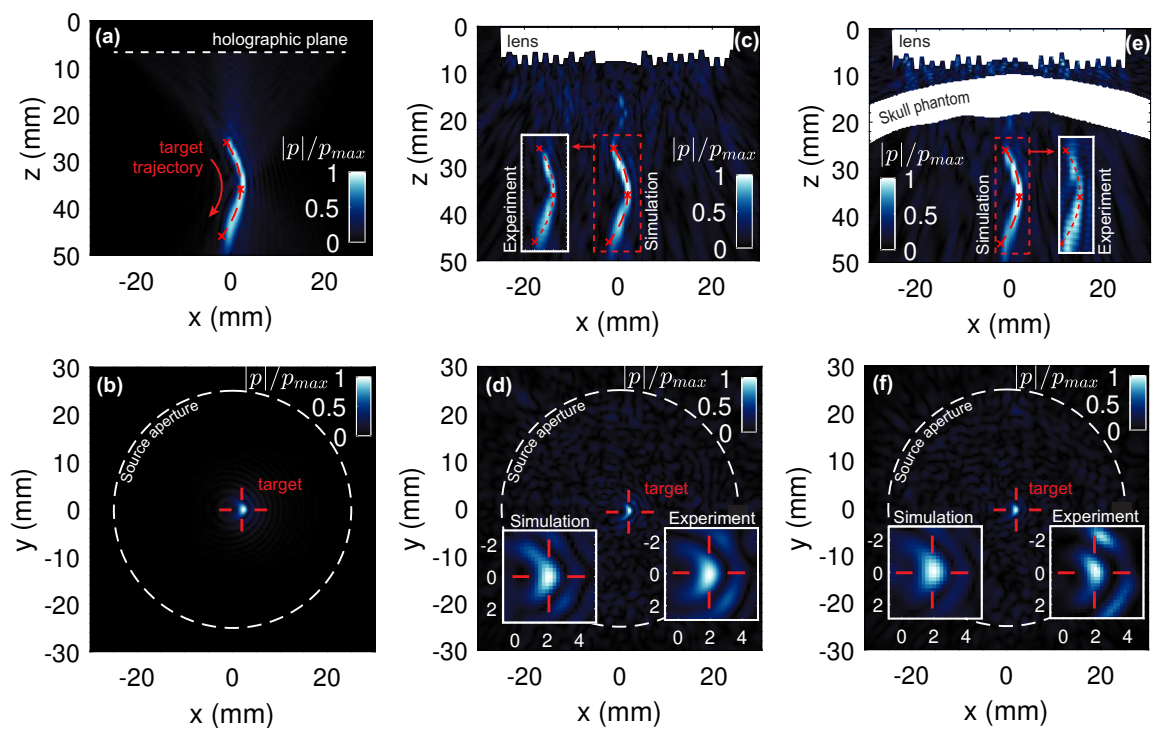

Figure 2.8: Theoretical (a) axial- and (b) transverse-pressure-field distribution for the self-bending beam in water. Color bar in units normalized to the peak pressure. Simulated (c) axial and (d) transverse-pressure-field distribution for the self-bending beam in water. Corresponding experimental results are shown in the insets in (c) and (d). (e),(f) Simulated axial and transversal pressure field, including the skull phantom. Corresponding experimental results are shown in the insets in (e),(f). 
the corresponding holographic lens also reconstructs the target acoustic image with a curved trajectory, as shown in Figs. 2.8 (e,f). A similar lateral shift of the peak pressure location in the experiments of $0.25 \mathrm{~mm}$ in the $x$-direction is observed at $z=30 \mathrm{~mm}$ and $y=0 \mathrm{~mm}$. The measured pressure field inside the skull phantom agrees with the simulation. Note that the transversal size of the curved beam at $z=30 \mathrm{~mm}$ is 1.11, 1.07, and 1.19 times the wavelength in water for the theoretical calculation, and for the simulations in water and including the skull phantom, respectively. Both results demonstrate that using TR methods, self-bending beams following a target curve can be obtained inside the skull phantom using acoustic holographic lenses.

\subsection{Volumetric holograms overlapping CNS struc- tures}

Going further, we design a holographic lens, which produces an acoustic image that fits the right human hippocampus volume. The holographic surface is placed near the occipital-parietal bones to adapt the acoustic image to the elongated geometry of the hippocampus. However, we locate the lens at the center of the skull symmetry plane in order to demonstrate the steering capabilities of this holographic lens. The lens generation process is based on the TR method with multiple virtual sources covering the target area (see Sec. 2.2 for further details). Figures 2.9 and 2.10 summarize the results for both water and including the aberration layer of the skull phantom, respectively.

On the one hand, Figs. 2.9 (a-c) show the forward-propagation field distribution of the holographic lens designed for water obtained using the theoretical, experimental, and simulation results, respectively. First, we observe a good agreement between experiments, simulation, and theory in both axial- [Figs. 2.9 (a-c)] and transversal-field distributions [Figs. 2.9 (f$\mathrm{h})$ ]. The beam is steered in the correct direction, and a broad focal spot is generated. The transversal- and axial-field cross-sections are shown in Figs. $2.9(\mathrm{~d}, \mathrm{e})$. The diffraction-limited image is reconstructed, and the field is enhanced mainly at the target volume. To quantify the performance, we define the overlapping volume as the overlapping volumes of the target region and the region of the acoustic pressure field under a threshold 

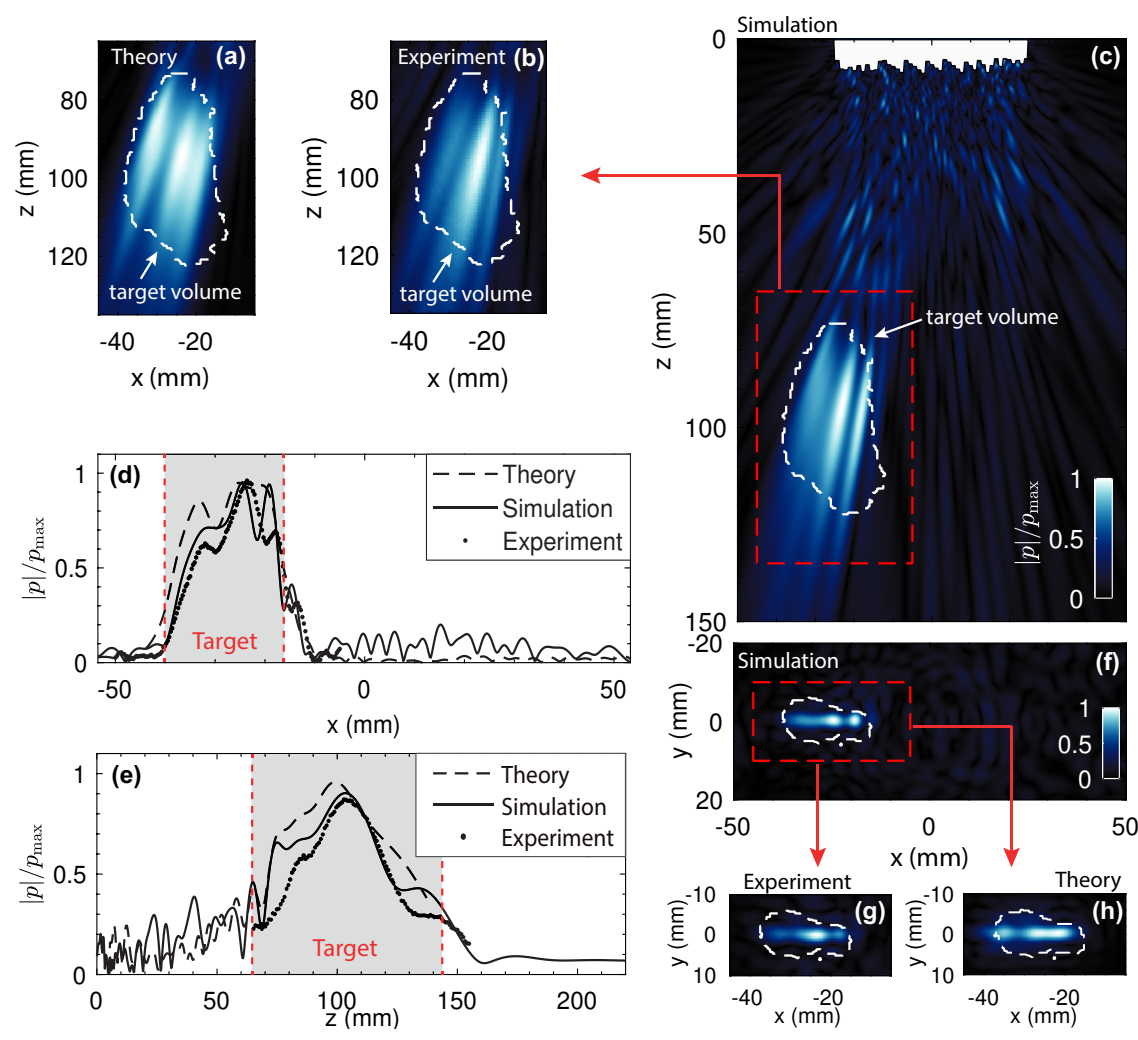

Figure 2.9: Volumetric hologram results in water. (a)-(c) Theoretical, experimental, and simulated axial pressure distribution. (d),(e) Transversal and axial field cross-sections. (f)-(h) Simulated, experimental, and theoretical transversal field distribution.

corresponding to the half of the peak amplitude. In particular, using this lens, we obtain, in water, an overlapping volume of 29.7, 20.1, and $19.0 \%$ for the theoretical calculation, simulation, and experiment, respectively.

On the other hand, the field distribution produced by acoustic holographic lenses including the skull phantom is shown in Figs. 2.10 (a-f). The experimental forward-propagation field distribution overlaps a similar volume in comparison with the simulation result. Both holographic images present the same qualitative performance and provide a similar 

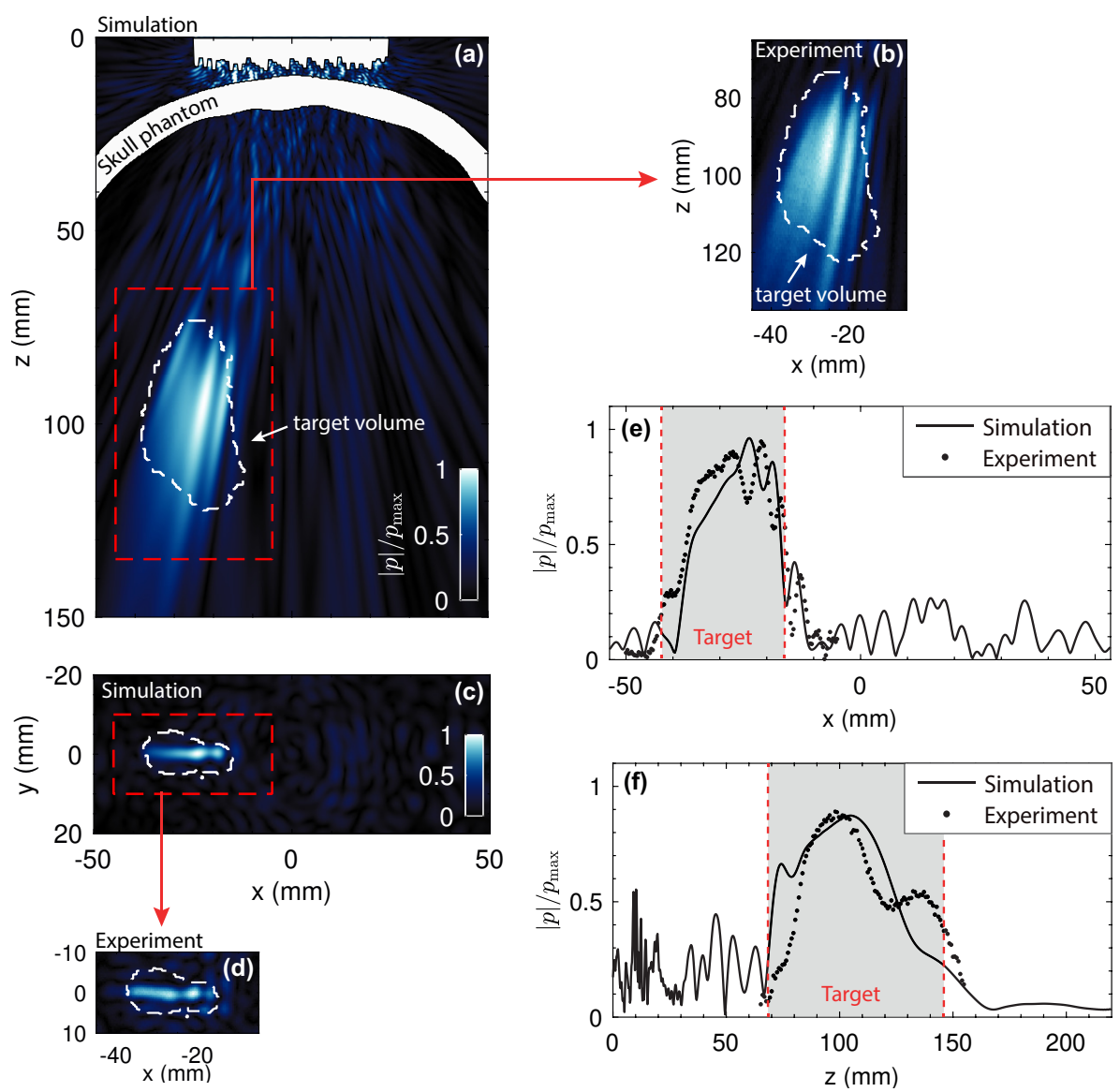

Figure 2.10: Volumetric hologram results with skull phantom. (a),(b) Simulated and experimental axial-pressure distribution. (c),(d) Simulated and experimental transversal-pressure distribution. (e),(f) Transversal and axial cross-sections, respectively.

overall covering of the interest zone. In particular, an overlapping volume of 21.1 and $23.2 \%$ is obtained in simulation and experiment, respectively. In addition, both axial- [Figs. 2.10 (a,b)] and transversal- [Figs. $2.10(\mathrm{c}, \mathrm{d})$ ] field distributions are similar to those produced in water without the skull phantom, showing that, first, limited-diffraction holographic volumes can 
be reconstructed and, second, the aberrations produced by the skull phantom on these complex beams can be compensated at the source plane by the acoustic holographic lenses. Finally, the transversal and axial crosssections, shown in Figs. 2.10 (e,f), show that the experimental and simulated acoustic holographic lens produces a field enhancement that matches the target distribution.

Note that the spatial bandwidth of the image is limited by the diffraction limit and the spatial bandwidth of the acoustic holographic lens [22]. In this case, the holographic lens focuses at $z \approx 74.1 \lambda(100 \mathrm{~mm})$, and its limited aperture is only $a=18.5 \lambda(25 \mathrm{~mm})$. Therefore, the transversal components of the wave vector are band-limited, and the performance of the holographic lens at this distance is restricted. Using lenses with a larger aperture improves the quality of the holographic acoustic image, and, therefore, the total overlapping volumes.

\subsection{Hologram simulation using a realistic skull}

It is worth noting here that the impedance of the available 3D printing material used to manufacture the skull phantom is soft compared with the skull bone. In this way, the phase aberrations produced by a real skull are stronger than those observed in the previous experiments. To demonstrate the focusing performance of the proposed lenses in a realistic situation, a set of simulations is performed using the acoustical parameters of skull bones. The parameters are derived using the same CT data and are listed in Sec. 2.2.4.

\section{Bifocal lens simulation using a realistic skull}

The results of the bifocal lens simulation using a realistic skull are summarized in Fig 2.11. First, the sagittal cross-section of the absolute value of the pressure field at $y=0 \mathrm{~mm}$ is shown in Fig. 2.11 (a). We can see that the lens focuses at two clear spots, almost at the target distance. The corresponding traversal cross-section is shown in Fig. 2.11 (b) measured at $z=100 \mathrm{~mm}$. In fact, good agreement is found between the simulations using a realistic skull and the calculations using the Rayleigh-Sommerfeld integral considering homogeneous water media. These two focal spots are 

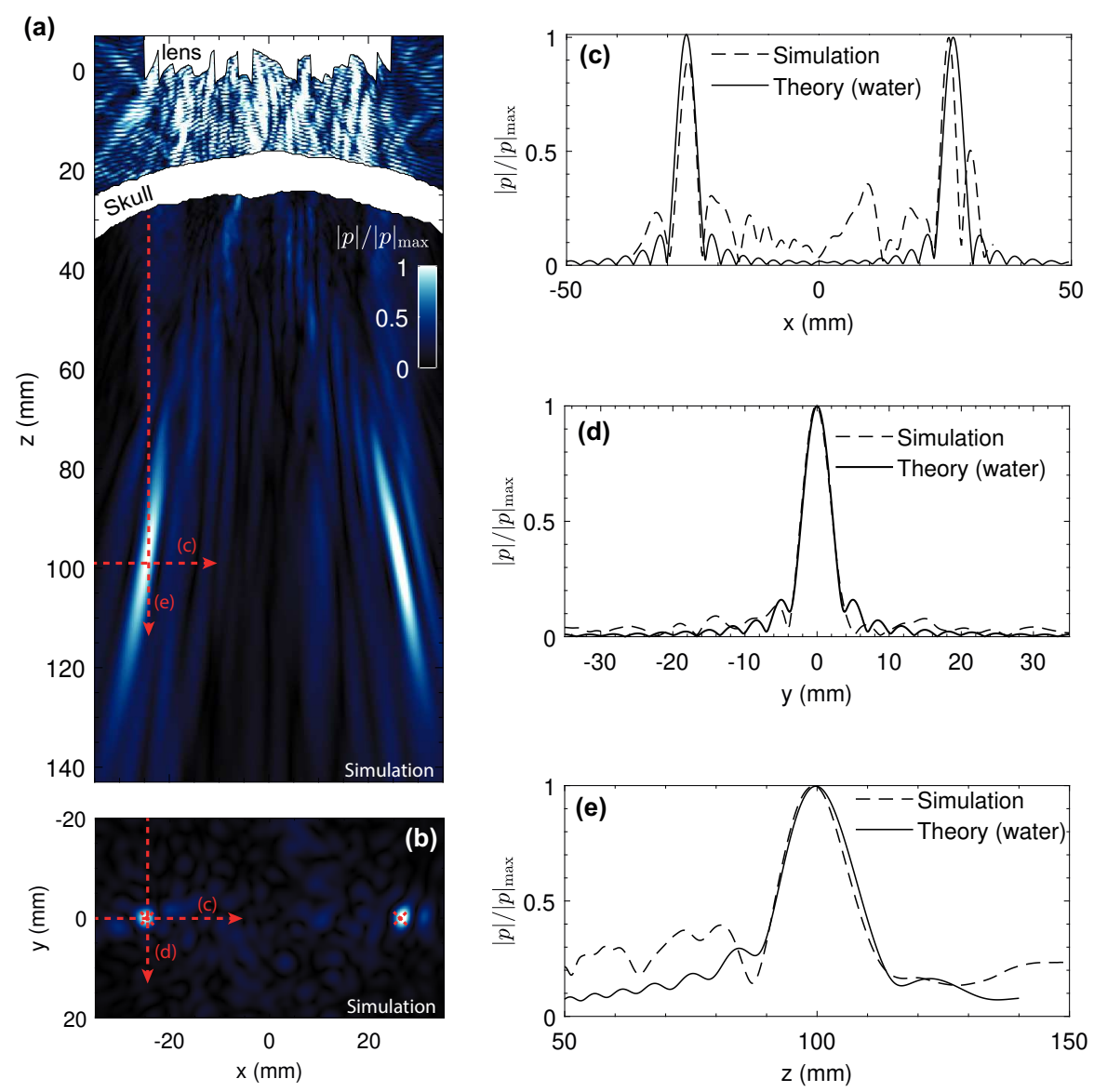

Figure 2.11: Simulation results for the bifocal holographic lens designed for a realistic skull. (a) Axial cross-section of the pressure distribution at $y=0 \mathrm{~mm}$. (b) Transversal cross-section at $z=100 \mathrm{~mm}$. (c) Lateral cross-section at $z=100 \mathrm{~mm}$ and $y=0 \mathrm{~mm}$. (d) Lateral cross-section at $z=100 \mathrm{~mm}$ and $x=-26 \mathrm{~mm}$. (e) Axial cross-section at $x=-26 \mathrm{~mm}$ and $y=0 \mathrm{~mm}$. 
generated together with small amplitude secondary lobes. The amplitude of the side lobes is $-8.86 \mathrm{~dB}$ below the peak pressure in theory in water and $-5.16 \mathrm{~dB}$ in the simulation including the skull. To quantify the focusing performance of the lens, we show in Fig. 2.11 (c) the transversal cross-section at $z=100 \mathrm{~mm}$ and $y=0 \mathrm{~mm}$. The lateral shift of the left focus is -26.3 and $-26.0 \mathrm{~mm}$ for the theoretical prediction and simulation, respectively. A relative error of $1.1 \%$ is committed. These small lateral shifts are of the order of the experimental test shown previously with the 3D-printed phantom. Moreover, the amplitude of the side lobes in the simulation using a realistic skull is 0.3 times the peak pressure. These side lobes present higher amplitude in the lateral cross-section joining both foci [Fig. 2.11 (c)] than in the lateral cross-section measured at $x=-26 \mathrm{~mm}$, as shown in Fig. 2.11 (d). Finally, Fig. 2.11 (e) shows the axial pressure distribution measured at the location of the right hippocampus. The axial peak location of the simulation including a realistic skull $(z=99.3 \mathrm{~mm})$ matches the location of the corresponding peak pressure using the theory in water $(z=99.8 \mathrm{~mm})$. A relative error of $0.5 \%$ is obtained, showing that the aberrations of a real skull can be mitigated using holographic lenses even when the target acoustic field presents a complex structure.

\section{Self-bending beam simulation inside a realistic skull}

The results for the self-bending beam simulation inside a realistic skull are shown in Fig. 2.12. The sagittal cross-section is shown in Fig. 2.12 (a), measured at $y=0 \mathrm{~mm}$, together with the location of the target marked in the red dashed line. In this case, the performance of the lens to produce such a complex beam is reduced as compared with the previous cases, as can be seen by the presence of secondary lobes. This is mainly caused by the generation of strong stationary waves between the skull bone and the lens. However, the peak pressure follows the target trajectory, and the location of the peak pressure matches the center of the curve. A clearer picture is given in Fig. 2.12 (b), which shows the transversal-field distribution measured at $z=35 \mathrm{~mm}$. Here, a sharp focal spot is visible, and the location of the peak pressure almost matches the location of the target focus. A lateral shift of $+0.22 \mathrm{~mm}$, which corresponds to one numerical grid step in the simulation is found in the $x$ direction. The corresponding transversal field distributions are shown in Fig. 2.12 (c) for the $x$-direction 
and in Fig. 2.12 (d) for the $y$-direction, respectively. Here, the reference calculations using theoretical methods in a homogeneous medium (water) are also shown for comparison. The locations of the focal spots observed in both lateral directions for the simulations including the realistic skull are in excellent agreement with the corresponding focal spots in water. The width of the focal spot obtained using both calculation methods also is in agreement. The main discrepancy is the presence of secondary lobes in the simulated field, presenting a peak amplitude of 0.36 times the pressure at the focus. The amplitude of these lobes is 1.7 times larger in the direction in which the beam is bent.

\section{Volumetric hologram using a realistic skull}

A holographic lens is designed with the target of producing a volumetric hologram overlapping with the right hippocampus volume, and in this case, including the acoustic properties of a realistic skull. The resulting forward-simulated pressure field is shown in Fig. 2.13. First, the sagittal cross section of the magnitude of the pressure field at $y=0 \mathrm{~mm}$ is shown in Fig. 2.13 (a). The produced field focuses around the target volume, shown in dashed white lines. The beam is steered in the direction of the right hippocampus while the transducer axis remains normal to the skull surface. The transversal cross-section at $z=95 \mathrm{~mm}$ is shown in Fig. 2.13 (b). While the acoustic field is focused on the target volume, there exist areas not covered by the beam, mainly in the outermost regions away from the transducer source. To quantify the focusing performance, field cross-sections along the corresponding dashed lines are given in Fig. 2.13 (c-e). The lateral cross-section at $z=95 \mathrm{~mm}$ and $y=0 \mathrm{~mm}$ is shown in Fig. 2.13 (c). As a comparison, we also plot the corresponding cross section of a holographic lens designed to produce the same hologram in water using the theoretical Rayleigh-Sommerfeld integration. The simulated beam using the realistic skull and the theoretical prediction in water mostly overlaps, being the energy of the beam concentrated into the target volume. Small side lobes with an amplitude 5.3 times smaller than the peak pressure are observed in the simulations including the realistic skull. Note that the corresponding acoustic intensity of the side lobes is about 30 times smaller than the intensity at the focus. The volume of the beam, defined as the total volume of the beam under a threshold of 0.5 times 
the peak pressure, roughly overlaps with the target volume. The overlapping volume between the target and the volumetric acoustic hologram is $29.7 \%$ for the theoretical calculation in water and $21.4 \%$ for the simulation including the skull. The transversal cross-section along the $y$-axis is given in Fig. 2.13 (d), where excellent agreement is found between the two configurations. Finally, the axial cross-section along the $z$-axis is shown in Fig. 2.13 (e). In this case, the field presents remarkable side lobes before and after the target region, but its amplitude is lower than half of the maximum pressure. Note that this direction corresponds to the beam axis
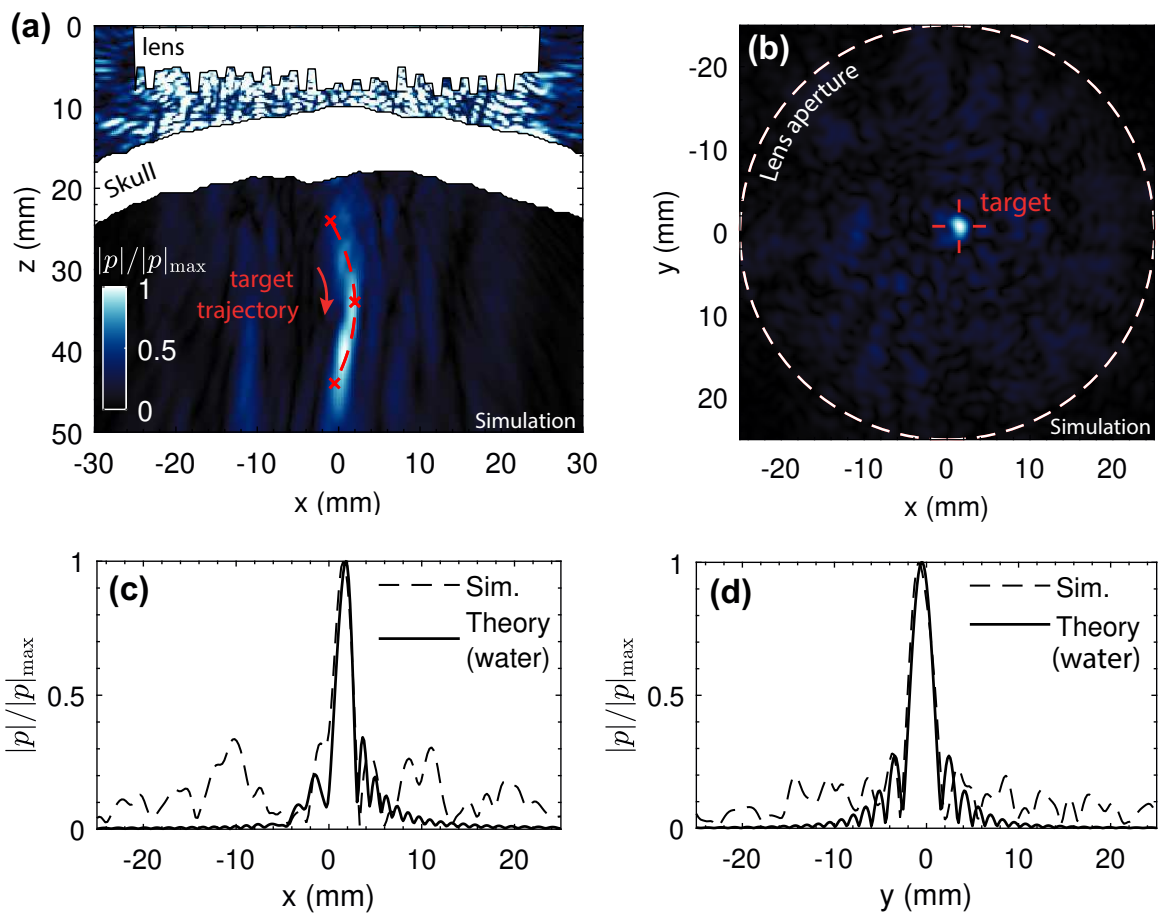

Figure 2.12: Simulation results for the self-bending holographic beam designed for a realistic skull. (a) Axial cross-section of the pressure distribution at $y=0 \mathrm{~mm}$. (b) Transversal cross-section at $z=35 \mathrm{~mm}$. (c) Lateral cross-section at $z=35 \mathrm{~mm}$ and $y=0 \mathrm{~mm}$. (d) Lateral cross-section at $z=35 \mathrm{~mm}$ and $x=2 \mathrm{~mm}$. 
and the pressure distribution of the corresponding focused beam presents an elongated shape due to the limited aperture of the source. In this case, a good agreement is also found between the axial-pressure distribution of the simulation, including the realistic skull, and the theoretical prediction in water.

\subsection{Conclusions}

We show that using 3D-printed acoustic holograms it is possible to conform diffraction-limited ultrasonic fields of arbitrary shape compensating the aberrations of a human skull. In particular, experimental tests using a 3D-printed skull phantom and numerical simulations using a realistic skull are performed to accurately generate multi-focal holograms, self-bending beams, and volumetric holographic fields overlapping a target CNS structure. The proposed approach using holographic lenses represents a step forward when compared with the existing solutions using phased arrays, since it opens alternative venues to develop reliable and cost-reduced ultrasonic applications.

The quality of the reconstructed acoustic images is related to the diffraction limit and the spatial bandwidth of the holographic lens, which depends on the spatial aperture of the lens, the number of pixels of the lens, and the frequency of the beam [22]. In this work, we target a human hippocampus using a single holographic lens of only 50-mm aperture and operating at a frequency of $1.1 \mathrm{MHz}$. The reported experimental results inside a skull phantom are in good agreement with theory and simulations. Only small shifts of the order of one wavelength (1.4 $\mathrm{mm}$ in water) are found between the target location and the field produced by the holographic lens. These shifts can be caused by experimental reasons that include small positioning errors between the lens and the curved surface of the phantom and can be corrected using optimization methods during lens design. It is worth noting here that the phantom used in the experiments presents a smaller acoustic impedance than a real skull. However, full-wave simulations performed using the density, sound speed, and attenuation values of the skull bone show that these arbitrary fields can also be produced in a realistic situation. The generated acoustic fields inside the skull are in good agreement with those produced in water. This 
shows that the aberrations produced by the skull can be mitigated by using holographic lenses even when a complex field is required.
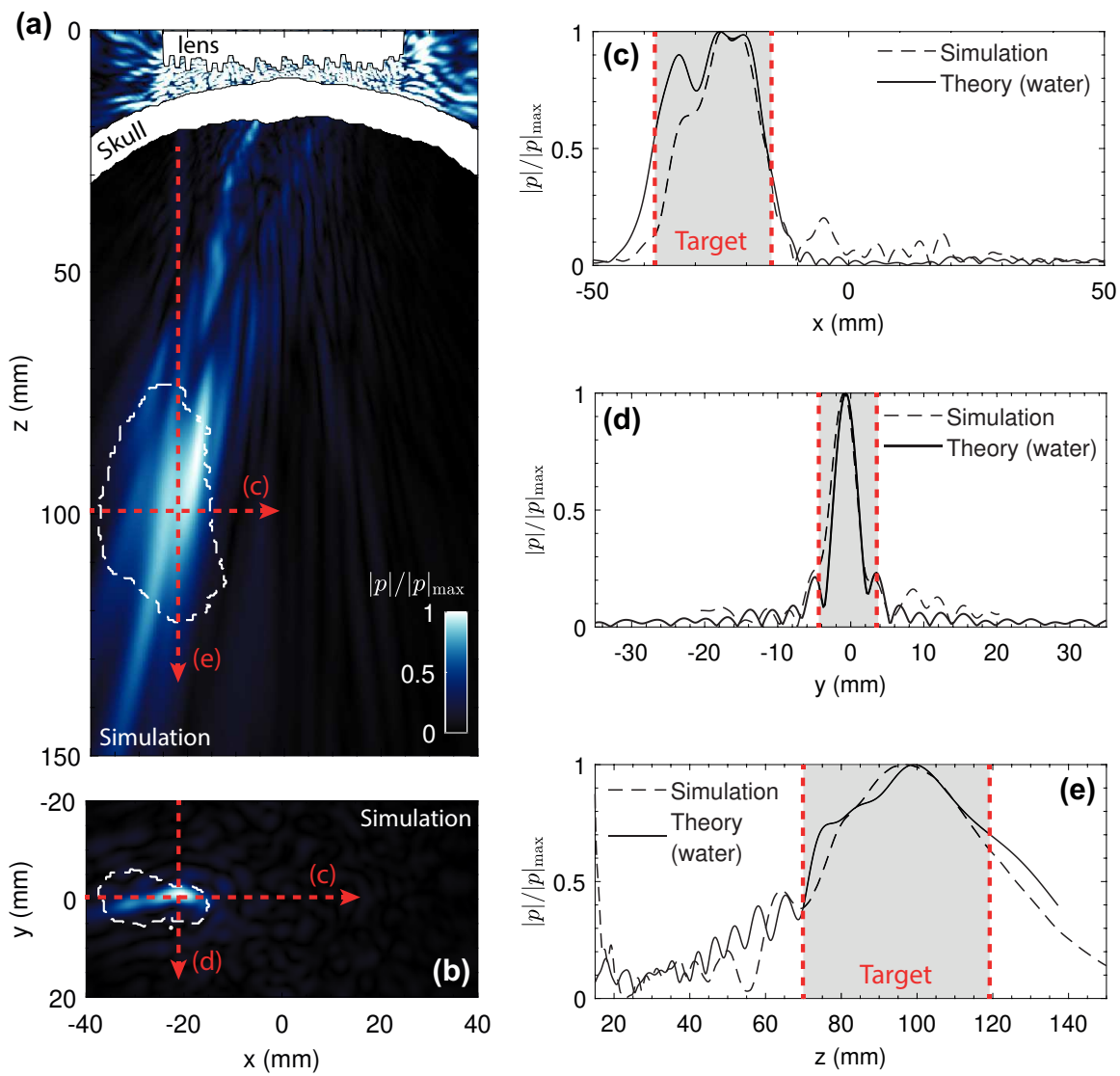

Figure 2.13: Simulation results for the volumetric hologram designed for a realistic skull. (a) Axial cross-section of the pressure distribution at $y=0 \mathrm{~mm}$. (b) Transversal cross-section at $z=95 \mathrm{~mm}$. (c) Lateral cross-section at $z=95 \mathrm{~mm}$ and $y=0 \mathrm{~mm}$. (d) Lateral cross-section at $z=95 \mathrm{~mm}$ and $x=-26 \mathrm{~mm}$. (e) Axial cross-section at $x=-26 \mathrm{~mm}$ and $y=0 \mathrm{~mm}$. 
Moreover, using the proposed methodology, diffraction is captured precisely compared with Fraunhofer or angular spectrum methods, leading to better accuracy of the generated acoustic fields. In addition, the holographic lens design is based on resonating slabs, which include not only the refraction over a curved plate, but the resonating waves inside the lens. Finally, it is important to remark that the holographic lenses used in this work capture the complete information of the wave field, both phase and amplitude are recorded and encoded in the phase-only lens. This approach allows us to reconstruct considerably complex fields.

Phased arrays are efficient, but their high cost can be prohibitive to spread out some of the incoming ultrasonic transcranial therapy treatments. Using phased arrays, the ultrasonic beams can be adjusted in real time and monitored using MRI, obtaining a precise location of the acoustic focus into the CNS. Nevertheless, the number of elements of the phased-array systems can be insufficient to produce a complex volumetric ultrasonic field that matches a specific CNS structure. The use of holographic lenses presents several advantages to produce complex volumetric patterns. First, the cost of a 3D-printed lens is low as compared with phased-array systems. Second, each pixel in a holographic lens acts as an element of the phased array with fixed phase. Due to the high number of passive sources in a holographic lens, more than 4000 for the small lenses considered here, complex patterns can be generated. Note that the complexity of the produced acoustic images can be improved using larger aperture lenses, i.e., increasing the angular spectrum of the recorded holographic information. However, once the lens is designed, its focal distance and the spatial features of the holographic image remain fixed, and, in principle, it is not possible to steer the ultrasonic beam in real-time with accuracy. For this reason, the technique is especially relevant for the treatment using a single sonification of structures, or a sonification of large volumes.

The concept shown in this chapter opens alternative doors to optimize and to disseminate incoming therapy treatments such as ultrasound-assisted blood-brain barrier opening for drug delivery and neuromodulation, or ultrasonic imaging of the central nervous system using low-cost devices. Considering the emergence of metamaterials and their huge flexibility, we also advance incoming biomedical applications of active holographic meta- 
surfaces for the generation of complex fields in the central nervous system.

\section{References - Chapter 2}

[1] T. E. Allibone, "Dennis gabor, 5 june 1900-9 february 1979," 1980.

[2] D. Gabor, "A new microscopic principle," Nature, vol. 161, pp. 777-778, 1948.

[3] D. Gabor et al., "Microscopy by reconstructed wave-fronts," Proc. R. Soc. Lond. A, vol. 197, no. 1051, pp. 454-487, 1949.

[4] E. N. Leith and J. Upatnieks, "Reconstructed wavefronts and communication theory," JOSA, vol. 52, no. 10, pp. 1123-1130, 1962.

[5] X. Ni, A. V. Kildishev, and V. M. Shalaev, "Metasurface holograms for visible light," Nature Communications, vol. 4, p. 2807, 2013.

[6] L. Huang, X. Chen, H. Mühlenbernd, H. Zhang, S. Chen, B. Bai, Q. Tan, G. Jin, K.-W. Cheah, C.-W. Qiu, et al., "Three-dimensional optical holography using a plasmonic metasurface," Nature Communications, vol. 4, p. $2808,2013$.

[7] G. Ma and P. Sheng, "Acoustic metamaterials: From local resonances to broad horizons," Science advances, vol. 2, no. 2, p. e1501595, 2016.

[8] S. A. Cummer, J. Christensen, and A. Alù, "Controlling sound with acoustic metamaterials," Nature Reviews Materials, vol. 1, p. 16001, 2016.

[9] Z. Liu, X. Zhang, Y. Mao, Y. Zhu, Z. Yang, C. T. Chan, and P. Sheng, "Locally resonant sonic materials," Science, vol. 289, no. 5485, pp. 17341736, 2000.

[10] N. Fang, D. Xi, J. Xu, M. Ambati, W. Srituravanich, C. Sun, and X. Zhang, "Ultrasonic metamaterials with negative modulus," Nature Materials, vol. 5, no. 6, p. $452,2006$.

[11] M. Yang, G. Ma, Z. Yang, and P. Sheng, "Coupled membranes with doubly negative mass density and bulk modulus," Physical Review Letters, vol. 110, no. 13, p. 134301, 2013.

[12] Y. Li, B. Liang, Z.-m. Gu, X.-y. Zou, and J.-c. Cheng, "Reflected wavefront manipulation based on ultrathin planar acoustic metasurfaces," Scientific Reports, vol. 3, p. 2546, 2013. 
[13] Y. Xie, W. Wang, H. Chen, A. Konneker, B.-I. Popa, and S. A. Cummer, "Wavefront modulation and subwavelength diffractive acoustics with an acoustic metasurface," Nature Communications, vol. 5, p. 5553, 2014.

[14] N. Jiménez, T. J. Cox, V. Romero-García, and J.-P. Groby, "Metadiffusers: Deep-subwavelength sound diffusers," Scientific Reports, vol. 7, no. 1, p. $5389,2017$.

[15] N. Jiménez, V. Romero-García, V. Pagneux, and J.-P. Groby, "Rainbowtrapping absorbers: Broadband, perfect and asymmetric sound absorption by subwavelength panels for transmission problems," Scientific Reports, vol. 7 , no. 1, p. $13595,2017$.

[16] S. Qi, Y. Li, and B. Assouar, "Acoustic focusing and energy confinement based on multilateral metasurfaces," Physical Review Applied, vol. 7, no. 5, p. 054006, 2017.

[17] E. Bok, J. J. Park, H. Choi, C. K. Han, O. B. Wright, and S. H. Lee, "Metasurface for water-to-air sound transmission," Physical review letters, vol. 120, no. 4, p. 044302, 2018.

[18] Y. Li, X. Jiang, B. Liang, J.-c. Cheng, and L. Zhang, "Metascreen-based acoustic passive phased array," Physical Review Applied, vol. 4, no. 2, p. $024003,2015$.

[19] Y. Li and M. B. Assouar, "Three-dimensional collimated self-accelerating beam through acoustic metascreen," Scientific reports, vol. 5, p. 17612, 2015.

[20] N. Kaina, F. Lemoult, M. Fink, and G. Lerosey, "Negative refractive index and acoustic superlens from multiple scattering in single negative metamaterials," Nature, vol. 525, no. 7567, p. 77, 2015.

[21] J. Li, L. Fok, X. Yin, G. Bartal, and X. Zhang, "Experimental demonstration of an acoustic magnifying hyperlens," Nature Materials, vol. 8, no. 12, p. 931, 2009 .

[22] K. Melde, A. G. Mark, T. Qiu, and P. Fischer, "Holograms for acoustics," Nature, vol. 537, no. 7621, p. 518, 2016.

[23] Y. Xie, C. Shen, W. Wang, J. Li, D. Suo, B.-I. Popa, Y. Jing, and S. A. Cummer, "Acoustic holographic rendering with two-dimensional metamaterialbased passive phased array," Scientific Reports, vol. 6, p. 35437, 2016. 
[24] Y. Zhu, J. Hu, X. Fan, J. Yang, B. Liang, X. Zhu, and J. Cheng, "Fine manipulation of sound via lossy metamaterials with independent and arbitrary reflection amplitude and phase," Nature communications, vol. 9, no. 1, p. $1632,2018$.

[25] G. Memoli, M. Caleap, M. Asakawa, D. R. Sahoo, B. W. Drinkwater, and S. Subramanian, "Metamaterial bricks and quantization of meta-surfaces," Nature communications, vol. 8, no. 1, pp. 1-8, 2017.

[26] M. D. Brown, B. T. Cox, and B. E. Treeby, "Design of multi-frequency acoustic kinoforms," Applied Physics Letters, vol. 111, no. 24, p. 244101, 2017.

[27] Y. Hertzberg and G. Navon, "Bypassing absorbing objects in focused ultrasound using computer generated holographic technique," Medical Physics, vol. 38, no. 12, pp. 6407-6415, 2011.

[28] P. Zhang, T. Li, J. Zhu, X. Zhu, S. Yang, Y. Wang, X. Yin, and X. Zhang, "Generation of acoustic self-bending and bottle beams by phase engineering," Nature Communications, vol. 5, p. 4316, 2014.

[29] A. Marzo, S. A. Seah, B. W. Drinkwater, D. R. Sahoo, B. Long, and S. Subramanian, "Holographic acoustic elements for manipulation of levitated objects," Nature Communications, vol. 6, p. 8661, 2015.

[30] M. A. Norasikin, D. Martinez Plasencia, S. Polychronopoulos, G. Memoli, Y. Tokuda, and S. Subramanian, "Soundbender: dynamic acoustic control behind obstacles," in Proceedings of the 31st Annual ACM Symposium on User Interface Software and Technology, pp. 247-259, 2018.

[31] . G. ter Haar and C. Coussios, "High intensity focused ultrasound: physical principles and devices," International journal of hyperthermia, vol. 23, no. 2, pp. 89-104, 2007.

[32] A. Bouakaz and J.-M. Escoffre, Therapeutic ultrasound: From biophysics concepts to clinical applications, vol. 880 of Advances in Experimental Medicine and Biology. Springer International Publishing, 2016.

[33] T. L. Szabo, Diagnostic ultrasound imaging: inside out. Academic Press, 2004.

[34] P. Gélat, G. Ter Haar, and N. Saffari, "A comparison of methods for focusing the field of a hifu array transducer through human ribs," Physics in Medicine E) Biology, vol. 59, no. 12, p. 3139, 2014. 
[35] F. Fry and J. Barger, "Acoustical properties of the human skull," The Journal of the Acoustical Society of America, vol. 63, no. 5, pp. 1576-1590, 1978.

[36] J.-L. Thomas and M. A. Fink, "Ultrasonic beam focusing through tissue inhomogeneities with a time reversal mirror: application to transskull therapy," IEEE Transactions on Ultrasonics, Ferroelectrics, and Frequency Control, vol. 43, no. 6, pp. 1122-1129, 1996.

[37] K. Hynynen and F. A. Jolesz, "Demonstration of potential noninvasive ultrasound brain therapy through an intact skull," Ultrasound in Medicine and Biology, vol. 24, no. 2, pp. 275-283, 1998.

[38] J. Sun and K. Hynynen, "Focusing of therapeutic ultrasound through a human skull: a numerical study," The Journal of the Acoustical Society of America, vol. 104, no. 3, pp. 1705-1715, 1998.

[39] J.-F. Aubry, M. Tanter, M. Pernot, J.-L. Thomas, and M. Fink, "Experimental demonstration of noninvasive transskull adaptive focusing based on prior computed tomography scans," The Journal of the Acoustical Society of America, vol. 113, no. 1, pp. 84-93, 2003.

[40] M. Tanter, J.-L. Thomas, and M. Fink, "Focusing and steering through absorbing and aberrating layers: Application to ultrasonic propagation through the skull," The Journal of the Acoustical Society of America, vol. 103, no. 5, pp. 2403-2410, 1998.

[41] Y. Hertzberg, A. Volovick, Y. Zur, Y. Medan, S. Vitek, and G. Navon, "Ultrasound focusing using magnetic resonance acoustic radiation force imaging: application to ultrasound transcranial therapy," Medical Physics, vol. 37, no. 6Part1, pp. 2934-2942, 2010.

[42] F. A. Jolesz, Intraoperative imaging and image-guided therapy. Springer Science \& Business Media, 2014.

[43] M. Pernot, J.-F. Aubry, M. Tanter, J.-L. Thomas, and M. Fink, "High power transcranial beam steering for ultrasonic brain therapy," Physics in Medicine E Biology, vol. 48, no. 16, p. 2577, 2003.

[44] C. Shen, J. Xu, N. X. Fang, and Y. Jing, "Anisotropic complementary acoustic metamaterial for canceling out aberrating layers," Physical Review $X$, vol. 4, no. 4, p. 041033, 2014.

[45] G. Maimbourg, A. Houdouin, T. Deffieux, M. Tanter, and J.-F. Aubry, "3dprinted adaptive acoustic lens as a disruptive technology for transcranial 
ultrasound therapy using single-element transducers," Physics in Medicine ES Biology, vol. 63, no. 2, p. 025026, 2018.

[46] M. Ferri, J. M. Bravo, J. Redondo, and J. V. Sánchez-Pérez, "Enhanced numerical method for the design of 3-d-printed holographic acoustic lenses for aberration correction of single-element transcranial focused ultrasound," Ultrasound in medicine 83 biology, vol. 45, no. 3, pp. 867-884, 2019.

[47] K. Hynynen, N. McDannold, N. Vykhodtseva, and F. A. Jolesz, "Noninvasive $\mathrm{mr}$ imaging-guided focal opening of the blood-brain barrier in rabbits," Radiology, vol. 220, no. 3, pp. 640-646, 2001.

[48] W. J. Tyler, Y. Tufail, M. Finsterwald, M. L. Tauchmann, E. J. Olson, and C. Majestic, "Remote excitation of neuronal circuits using low-intensity, low-frequency ultrasound," PloS one, vol. 3, no. 10, p. e3511, 2008.

[49] U. Schneider, E. Pedroni, and A. Lomax, "The calibration of ct hounsfield units for radiotherapy treatment planning," Physics in Medicine $\&$ Biology, vol. 41, no. 1, p. 111, 1996.

[50] T. D. Mast, "Empirical relationships between acoustic parameters in human soft tissues," Acoustics Research Letters Online, vol. 1, no. 2, pp. 37-42, 2000 .

[51] J. C. Mazziotta, A. W. Toga, A. Evans, P. Fox, J. Lancaster, et al., "A probabilistic atlas of the human brain: theory and rationale for its development," Neuroimage, vol. 2, no. 2, pp. 89-101, 1995.

[52] P. A. Yushkevich, J. Piven, H. C. Hazlett, R. G. Smith, S. Ho, J. C. Gee, and G. Gerig, "User-guided 3d active contour segmentation of anatomical structures: significantly improved efficiency and reliability," Neuroimage, vol. 31, no. 3, pp. 1116-1128, 2006.

[53] D. T. Blackstock, "Fundamentals of physical acoustics," 2001.

[54] K. Matsushima and T. Shimobaba, "Band-limited angular spectrum method for numerical simulation of free-space propagation in far and near fields," Optics express, vol. 17, no. 22, pp. 19662-19673, 2009.

[55] B. E. Treeby and B. Cox, "Modeling power law absorption and dispersion for acoustic propagation using the fractional laplacian," The Journal of the Acoustical Society of America, vol. 127, no. 5, pp. 2741-2748, 2010. 
[56] B. E. Treeby, J. Jaros, A. P. Rendell, and B. Cox, "Modeling nonlinear ultrasound propagation in heterogeneous media with power law absorption using ak-space pseudospectral method," The Journal of the Acoustical Society of America, vol. 131, no. 6, pp. 4324-4336, 2012.

[57] N. Jiménez, F. Camarena, J. Redondo, V. Sánchez-Morcillo, Y. Hou, and E. E. Konofagou, "Time-domain simulation of ultrasound propagation in a tissue-like medium based on the resolution of the nonlinear acoustic constitutive relations," Acta Acustica united with Acustica, vol. 102, no. 5, pp. 876$892,2016$.

[58] N. Jiménez, V. Romero-García, V. Pagneux, and J.-P. Groby, "Quasiperfect absorption by subwavelength acoustic panels in transmission using accumulation of resonances due to slow sound," Physical Review B, vol. 95, no. 1, p. 014205, 2017.

[59] J. Allard and N. Atalla, Propagation of sound in porous media: modelling sound absorbing materials 2e. John Wiley \& Sons, 2009.

[60] P. W. M. Tsang and T.-C. Poon, "Novel method for converting digital fresnel hologram to phase-only hologram based on bidirectional error diffusion," Optics Express, vol. 21, no. 20, pp. 23680-23686, 2013.

[61] R. Lirette and J. Mobley, "Focal zone characteristics of stepped fresnel and axicon acoustic lenses," Proceedings of Meetings on Acoustics, vol. 31, no. 1, p. 045001, 2017.

[62] M. Gatto, G. Memoli, A. Shaw, N. Sadhoo, P. Gelat, and R. A. Harris, "Three-dimensional printing (3dp) of neonatal head phantom for ultrasound: Thermocouple embedding and simulation of bone," Medical Engineering and Physics, vol. 34, no. 7, pp. 929-937, 2012.

[63] J. Robertson, E. Martin, B. Cox, and B. E. Treeby, "Sensitivity of simulated transcranial ultrasound fields to acoustic medium property maps," Physics in Medicine ES Biology, vol. 62, no. 7, p. 2559, 2017.

[64] C. Bai, M. Ji, A. Bouakaz, Y. Zong, and M. Wan, "Design and characterization of an acoustically and structurally matched 3d-printed model for transcranial ultrasound imaging," IEEE Transactions on Ultrasonics, Ferroelectrics, and Frequency Control, 2018.

[65] C. R. Hill, J. Bamber, and G. Ter Haar, Physical principles of medical ultrasonics. John Wiley \& Sons New York-Chichester-Brisbane-Toronto, 2004 . 
[66] R. S. Cobbold, Foundations of biomedical ultrasound. Oxford University Press, 2006.

[67] H. O'Neil, "Theory of focusing radiators," The Journal of the Acoustical Society of America, vol. 21, no. 5, pp. 516-526, 1949.

[68] D.-C. Chen, X.-F. Zhu, Q. Wei, D.-J. Wu, and X.-J. Liu, "Broadband acoustic focusing by airy-like beams based on acoustic metasurfaces," Journal of Applied Physics, vol. 123, no. 4, p. 044503, 2018.

[69] Cover image for this chapter and for the dissertation book, creator name: immimagery, stock.adobe.com.

[70] Cover image for the dissertation book background, creator name: YustynaOlha, stock.adobe.com.

\section{Publications}

The contents of this chapter, and closely related ones, were presented in the following publications:

\section{Journal papers}

- S. Jiménez-Gambín, N. Jiménez, J. M. Benlloch, and F. Camarena, "Holograms to focus arbitrary ultrasonic fields through the skull". Physical Review Applied, vol. 12, no. 1, p. 014016, 2019.

- M. Ferri, J. M. Bravo, J. Redondo, S. Jiménez-Gambín, N. Jiménez, F. Camarena, and J. V. Sánchez-Pérez, "On the evaluation of the suitability of the materials used to 3D print holographic acoustic lenses to correct transcranial focused ultrasound aberrations". Polymers, vol. 11, no. 9, p. 1521, 2019.

- S. Jiménez-Gambín, N. Jiménez, and F. Camarena, "Efecto del método de definición de las propiedades acústicas de cráneo humano en la propagación focalizada de ultrasonidos". Revista de Acústica, vol. 50, no. 1-2, p. 20-24, 2019. 


\section{Conference proceedings}

- S. Jiménez-Gambín, N. Jiménez, Shih-Ying Wu, E. E. Konofagou, and F. Camarena, "Study of aberrations at the focus of an ultrasonic beam due to the propagation across different areas of the skull". 5th International Symposium on Focused Ultrasound, Maryland, USA. August 28 September 13, 2016.

- S. Jiménez-Gambín, N. Jiménez, M. Company, and F. Camarena, "Estudio numérico del protocolo de propagación transcraneal de ultrasonidos para la apertura de la barrera hematoencefálica en el hipocampo de humano". $48^{\circ}$ Congreso Español de Acústica; Encuentro Ibérico de Acústica; European Symposium on Underwater Acoustics Applications; European Symposium on Sustainable Building Acoustics; TECNIACUSTICA 2017, A Coruña, Galicia, Spain. October 4-6, 2017.

- S. Jiménez-Gambín, N. Jiménez, and F. Camarena, "Efectos del método de obtención de las propiedades acústicas de cráneo humano en la propagación focalizada de ultrasonidos". $49^{\circ}$ Congreso Español de Acústica; XI Congreso Iberoamericano de Acústica; X Congreso Ibérico de Acústica; TECNIACUSTICA 2018, Cádiz, Andalucía, Spain. October 24-26, 2018. Award: Luis de Camoens UC3M Prize, award for the best scientifictechnical communication presented by a young researcher.

- F. Camarena, S. Jiménez-Gambín, N. Jiménez, and J. M. Benlloch, "Ultrasonic Holograms in the Brain". VI Mediterranean Thematic Workshop in Advanced Molecular Imaging: Imaging in immunotheray (MEDAMI), Valencia, Comunidad Valenciana, Spain. May 15-17, 2019.

- S. Jiménez-Gambín, N. Jiménez, A. Marin, and F. Camarena, "Optimal Overlapping Protocol and Robustness Assessment of Blood-Brain Barrier Opening in Humans Using a Single-Element Focused Ultrasound Transducer". 19th International Symposium of ISTU, Barcelona, Cataluña, Spain. June 13-15, 2019.

- S. Jiménez-Gambín, N. Jiménez, J. M. Benlloch, and F. Camarena, "Acoustic Holograms for Transcranial Focusing of Arbitrary Ultrasonic Fields into the Brain". 19th International Symposium of ISTU, Barcelona, Cataluña, Spain. June 13-15, 2019.

- N. Jiménez, S. Jiménez-Gambín, J. M. Benlloch, and F. Camarena, "Focused ultrasound beyond phase-arrays: acoustic holographic lenses enable transcranial focusing of arbitrary fields at the central nervous system". XXXVII Reunión bienal de la Real Sociedad Española de Física, Zaragoza, Aragón, Spain. July 15-19, 2019. 
- S. Jiménez-Gambín, N. Jiménez, J. M. Benlloch, and F. Camarena, "Transcranial acoustic holograms for arbitrary fields generation using focused ultrasound into the brain". International Congress on Ultrasonics (ICU), Bruges, Belgium. September 3-6, 2019.

- S. Jiménez-Gambín, N. Jiménez, J. M. Benlloch, and F. Camarena, "Transcranial focusing of arbitrary ultrasonic fields using acoustic holograms". 23rd International Congress on Acoustics (ICA) integrating 4th EAA Euroregio, Aachen, Germany. September 9-13, 2019.

- S. Jiménez-Gambín, N. Jiménez, J. M. Benlloch, and F. Camarena, "Acoustic holograms allow the generation of complex fields inside the central nervous system". IEEE International Ultrasonics Symposium (IUS), Glasgow, Scotland, United Kingdom. October 6-9, 2019.

- D. Andrés, S. Jiménez-Gambín, N. Jiménez, and F. Camarena, "Multifocal acoustic holograms for ultrasound focusing at deep brain structures". 51 Congreso Español de Acústica; TECNIACUSTICA 2020; XI Congreso Ibérico de Acústica, virtual congress. June 3-5, 2020. Award: Andrés Lara Prize, ex aequo award for young researchers in its eighteenth edition.

- D. Andrés, S. Jiménez-Gambín, N. Jiménez, and F. Camarena, "Multifocal acoustic holograms for deep-brain neuromodulation and BBB opening". IEEE International Ultrasonics Symposium (IUS), virtual symposium. September 7-11, 2020.

- D. Andrés, S. Jiménez-Gambín, N. Jiménez, and F. Camarena, "Transcranial multifocal acoustic holograms". 7th International Symposium on Focused Ultrasound, virtual symposium. November 8-13, 2020. 




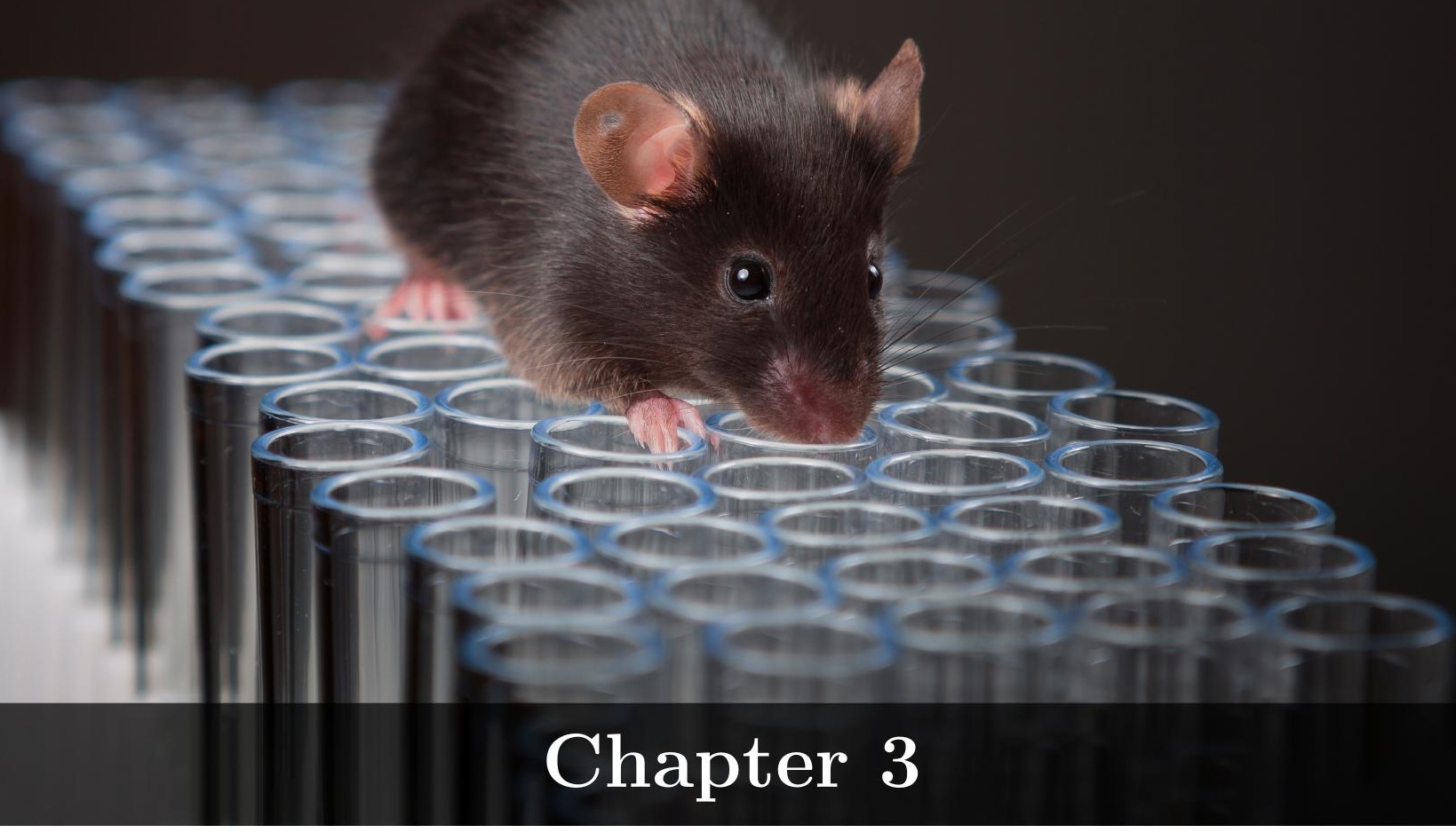

\section{Validation of acoustic holograms in small laboratory animals}

Once acoustic holograms worked in simulations, and experiments with a skull phantom, this third chapter shows a realistic BBB opening scenario. We achieved a simultaneous bilateral BBB opening in anesthetized mice in vivo. This approach showed three main advantages: simple and low-cost; aberrations corrected; and a single sonication enabled multiple BBB opening locations. MRI showed two symmetric BBB opening regions. We report repeatability of bilateral $\mathrm{BBB}$ opening in different mice by designing a unique lens from a unique $\mu \mathrm{CT}$ scan. This work demonstrates the feasibility of hologram-assisted BBB opening as a time- and cost-effective technique for multi-target drug delivery into the CNS. 


\subsection{Introduction}

The current technology for BBB opening using ultrasound relies on small implantable intracranial transducers, single-element FUS transducers or phased-array FUS transducers. However, these conventional methods consist of targeting only a single location at a time, not being able to target a set of locations simultaneously. On the one hand, the existing methods using a single-element transducer in small animals consist of a reduced MRI-compatible mechanism that mechanically moves the transducer to achieve the multi-focal targeting inside the brain of the rodent [1-6]. This mechanical movement is a limitation because the microbubbles are rapidly diluted into the bloodstream and filtered out from the lungs and liver. Therefore, several injections are required to sonicate multiple locations. Furthermore, a dedicated control MRI-scan is carried out for each target location to ensure that the ultrasound is focused on the desired brain spot or structure. Consequently, this is a time- and cost-inefficient approach for the multi-focal BBB opening in small animals. Besides, using neuronavigation systems for the controlled targeting in non-human primates [7] requires several injections of microbubbles as well, in case of a multi-focal or large-volume $\mathrm{BBB}$ opening procedure, because the mechanical movement of the transducer is a time-limiting factor. On the other hand, phased-array systems used in clinical trials $[8,9]$ have shown the ability to electronically and independently control the magnitude and phase of each piezoelectric element in order to change the focal spot location, keeping the transducer fixed in the same position during the whole sonication procedure. However, these systems have a limited steering capability away from the geometric focus [10], and do not allow either to sonicate large volumes with a single injection of microbubbles because their controllers take time to reach the functional pressure for each target, and by then, the microbubbles have been filtered out. Moreover, MRI-guided multi-element transducers are of high cost and not affordable for wide clinical use.

Recently, acoustic holograms have been proposed for transcranial FUS to overcome these cost- and time-limitations [11]. An acoustic hologram is a material designed to modulate the phase spatially, and, in some cases, the magnitude of a transmitted wavefront to synthesize a complex acoustic field [12-17], as shown in the previous chapter. These structures allow 
the synthesis of acoustic images, i.e., areas where the acoustic energy is high, combined with areas where the media is at rest. The technique of coupling an acoustic holographic lens to a single-element ultrasound transducer allows the generation of pressure fields of arbitrary shape, from simple focused beams $[18,19]$ to multi-focal spots or acoustic images matching the focal volume with the geometry of specific CNS structures [11] or complex distributions[20-22], self-bending beams [11], acoustic vortex beams [23, 24] or, recently, complex cavitation patterns [25]. Moreover, acoustic holographic lenses can be designed to compensate for the strong phase aberrations of the wavefront produced by the refraction and attenuation of the skull $[11,18,24,26]$. Multi-focal acoustic holograms are a low-cost alternative to avoid the time-inefficient mechanical movement of a single-element transducer [7] or single-spot lenses [19].

Acoustic holograms have been tested in water tanks, in phantoms, or using ex vivo human skulls, but to date, they have not been applied in vivo to produce a therapeutic effect. In this work, we demonstrate for the first time the capability of multi-focal acoustic holographic lenses to perform a bilateral BBB opening in mice (a set of $n=3$ mice are tested). The proposed method consists of a bifocal acoustic hologram coupled to a single-element tFUS transducer, as sketched in Fig. 3.1, designed to target both hemispheres in the brain of a mouse simultaneously. A passive cavitation detector was introduced to monitor the therapy. In addition to focusing simultaneously at different brain areas, this lens corrects the aberrations due to the skull, the brain, the coupling system, and the central hole for the imaging transducer. Using this low-cost instrumentation, we demonstrate the capability, feasibility, and efficiency to produce a therapeutic effect in a preclinical animal model.

\subsection{Methods}

The procedure of $\mathrm{BBB}$ opening using a holographic lens is divided into five steps. First, we extract the geometry and acoustic properties of a separate mouse skull from X-ray $\mu \mathrm{CT}$ images, and we identify the target locations using MRI scans. Second, a plastic cone for the water coupling between the transducer and the head of the rodent is designed and 3D-printed. Third, a simulation is performed to calculate the acoustic field back-propagated 
(a)

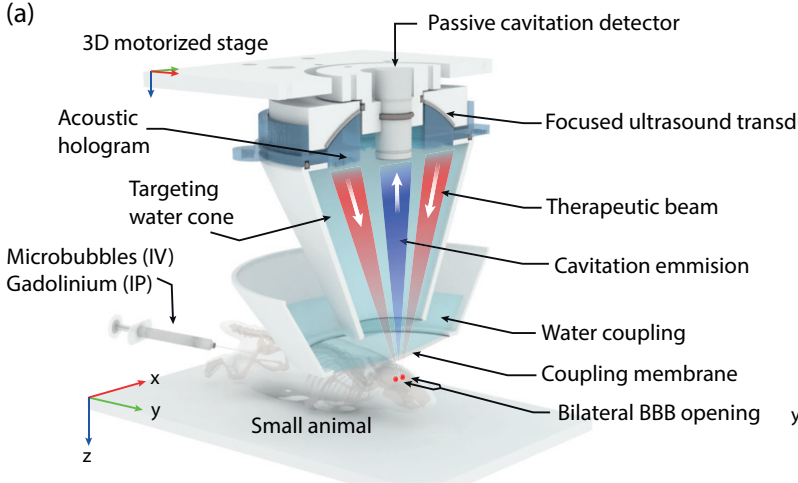

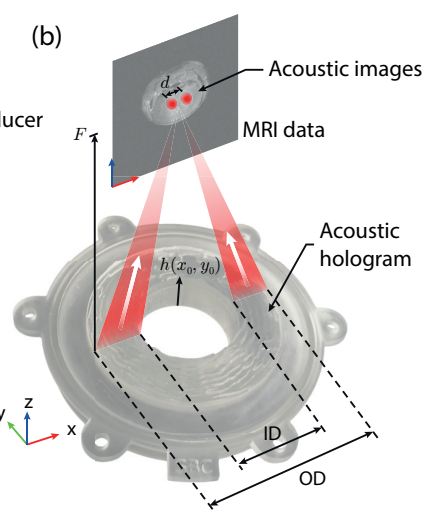

Figure 3.1: (a) Scheme of the preclinical therapeutic device using a bifocal acoustic hologram. Microbubbles were injected intravenously (IV) for the FUS delivery, while the gadolinium was injected intraperitoneally (IP) post-FUS delivery for the MRI procedure. (b) Photograph of the hologram manufactured by stereo-lithographic 3D-printing techniques using translucent resin, and geometrical parameters of the study. The roughness of the hologram is given by the function $h\left(x_{0}, y_{0}\right)$.

from the targets to the holographic surface. This simulation includes the multiple reflections and phase changes due to both the skull bones and the coupling cone. Fourth, the holographic acoustic lens is designed using the phase-conjugation of the back-propagated field, manufactured using stereo-lithographic 3D-printing, and attached to a single-element FUS transducer, as shown in Fig. 3.1 (a-b). Fifth, microbubbles are injected to the mouse and, then, ultrasound is delivered to the mouse head. At the same time, the treatment is monitored by measuring cavitation activity using a single-element passive-cavitation detector. Finally, MRI scans are carried out to assess the BBB opening.

\subsubsection{Numerical simulation}

We use a pseudo-spectral simulation method with $k$-space dispersion correction to numerically integrate the linearised constitutive relations of acoustics, as explained in Chapter 2 [see Sec. 2.2.2]. We use an isotropic 
numerical grid with a spatial step of $\Delta h=176 \mu \mathrm{m}$, which corresponds to a spatial sampling of five grid points per wavelength in water for a frequency of $1.68 \mathrm{MHz}$, leading to simulations of more than 300-million grid points. The numerical temporal step $\Delta t$ is set to $5.1 \mathrm{~ns}$ and $13.7 \mathrm{~ns}$ for the simulations with and without the head of the animal (particularly skull and brain media), respectively, leading to a Courant-Friedrichs-Lewy number of 0.2 in both configurations.

\subsubsection{Mouse skull acquisition for simulation}

The geometry and properties of the skull were acquired by $\mu \mathrm{CT}$-scans (R_mCT2, Rigaku, Tokyo, Japan) from a single mouse (mass: $24 \mathrm{~g}$, age: 8 weeks, sex: male, C57BL/6, Harlan, Indianapolis, IN, USA) with an isotropic spatial resolution of $80 \times 80 \times 80 \mu \mathrm{m}$, extrapolated to $17 \mu \mathrm{m}$ isotropic for the numerical simulation. It is assumed that the holographic acoustic lens designed from this single $\mu$ CT-scan can correct the skull aberrations of different but similar individuals ( $n=3$ mice). Thereby, we can assess the reproducibility of a single lens design for several mice, as well as speed up the BBB opening procedure for all mice. The heterogeneous acoustical properties of the skull are derived as described in Chapter 2 [see Sec. 2.2.1]. The absorption of the mouse skull is assumed to be homogeneous [27-29], and it has been set to $28.3 \mathrm{~dB} / \mathrm{cm}$ at $1.68 \mathrm{MHz}$ [30] with a frequency-power law exponent of $\gamma=1$. Afterward, the brain volume is segmented from the cavity in the $\mu \mathrm{CT}$-scan using the ITK-SNAP software [31]. Acoustical properties of the mouse brain are also included in the simulations, using $\rho_{\text {brain }}=1000 \mathrm{~kg} / \mathrm{m}^{3}, c_{\text {brain }}=1600 \mathrm{~m} / \mathrm{s}$ and an absorption coefficient of $0.7 \mathrm{~dB} / \mathrm{cm}$ at $1.68 \mathrm{MHz}$ [32] with $\gamma=1$.

\subsubsection{Lens design}

The lens was designed to produce a time-reversed version of the wavefront diverging from the two target points, following the procedure described in Chapter 2, Sec. 2.2.3, for flat transducers, modified here to be used in a focused ultrasound source. The single-element FUS transducer used was the Sonic Concepts H-204 (central frequency of $1.68 \mathrm{MHz}$, aperture of $O D=84.2 \mathrm{~mm}$, internal hole diameter of $I D=43.6 \mathrm{~mm}$, and radius of curvature of $F_{0}=61.65 \mathrm{~mm}$ ). First, the two target regions 
inside the mouse brain were transversely identified following the procedure described in Refs. [33-35], corresponding to two symmetric targets placed one in each brain hemisphere generally used for small animal models. The beam covered the hippocampus, thalamus, and hypothalamus of both hemispheres. Furthermore, to demonstrate the focusing capabilities of the hologram, we selected a focusing depth for the hologram $(F=100 \mathrm{~mm})$ different than the natural radius of curvature of the source $\left(F>F_{0}\right)$. Then, we located a virtual monopole source in the simulation method at the center of each target. As we were using a spherically-focused transducer, the lens had to compensate for the natural curvature of the wavefront. In addition, the central hole, the passive cavitation detector (PCD) used for monitoring the cavitation activity, and the plastic coupling cone would generate reflections that can be compensated by the hologram [36]. Therefore, the complete holographic information to design the lens is calculated by

$$
H_{L}(x, y)=H_{T}^{*}(x, y) H_{F}^{*}(x, y)
$$

where $H_{T}^{*}(x, y)$ is the complex conjugate of the recorded wavefront at the holographic surface due to the effects of the two virtual target sources, the plastic coupling cone, the skull, and the brain, $H_{F}^{*}(x, y)$ is the complex conjugate of the recorded wavefront at the holographic surface generated by the FUS transducer, including its diffraction at the border and at the central hole where the PCD is located, $(\cdot)^{*}$ denotes complex conjugation, and $x$ and $y$ are the transversal coordinates along the holographic plane.

Then, a phase-only lens was designed using the phase of the total complex holographic information (as explained in Chapter 2, Sec. 2.2.3. As a result, we obtained the surface of the hologram for the focused ultrasound source. Both, the hologram and the coupling cone were 3D-printed using ClearResin, a photosensitive polymer for stereolithography using a Form 2 printer (FormLabs, Somerville, MA, USA). For this material, we experimentally measured in a cylinder test piece, using pulse-echo techniques, a sound speed of $c_{L}=2580 \mathrm{~m} / \mathrm{s}$, a density of $\rho_{L}=1171 \mathrm{~kg} / \mathrm{m}^{3}$ and an absorption of $\alpha=4.6 \mathrm{~dB} / \mathrm{cm}$ at $1.68 \mathrm{MHz}$, in agreement with the values reported in the literature $[11,12]$. 


\subsubsection{Guiding and monitoring system}

The FUS transducer and the lens were confocally mounted with the Olympus V312-SU ultrasound imaging transducer (central frequency of $10 \mathrm{MHz}$, aperture of $\mathrm{OD}=6 \mathrm{~mm}$, flat active element), as shown in Fig. 3.1 (a). This imaging device was used in pulse-echo mode to align the system at the beginning of the procedure. A metallic grid placed on top of the lamboid suture of the skull was used as a reference, the system was aligned and, then the grid was removed. The imaging transducer was driven by a pulser-receiver system (Panametrics, Waltham, MA, USA), which was connected to a digitizer (Gage Applied Technologies, Inc., Lachine, QC, CAN) integrated into a personal computer (PC, Dell Inc., TX, USA). The same imaging transducer was used in reception mode as a PCD during treatment to detect the cavitation activity of the microbubbles under the action of the therapeutic ultrasound field. Acoustic cavitation emissions were processed offline in MATLAB ${ }^{\circledR}$ (The MathWorks, Natick, MA, USA).

Assuming that the PCD presents a linear response between the pressure at its surface, $p(t)$, and the recorded voltage, $V(t)$, the acoustic energy density, $W$, detected by the probe is proportional to

$$
W \approx \frac{1}{T} \int_{0}^{T} \frac{p^{2}}{\rho_{0} c_{0}^{2}} d t \sim \frac{1}{T} \int_{0}^{T} V^{2} d t \approx \frac{1}{T} \sum_{t=0}^{T} V^{2} \Delta t,
$$

where $V(t)$ is the voltage measured at each time point in Volts and $\Delta t$ was the sampling period equal to $10 \mathrm{~ns}$ [37]. Then, frequency analysis was conducted to identify the cavitation dose at each sonication $[7,38]$.

\subsubsection{Sonication and post-MRI procedure}

For the in vivo experiment, $1 \mu \mathrm{l} / \mathrm{g}$ Definity microbubbles were injected intravenously and FUS was applied for a total of 2 minutes using a $1.68-\mathrm{MHz}$ sinusoidal pulsed-burst of $400 \mathrm{kPa}$ peak-negative pressure (PNP), a pulse repetition frequency (PRF) of $5 \mathrm{~Hz}$, and a pulse length of $1 \mathrm{~ms}$. Then, $0.2 \mathrm{ml}$ of gadolinium tracer was injected intraperitoneally and post-treatment in vivo BBBO was assessed using T1-weighted MRI. Mice were transferred to the MRI scanner, anesthetized with 1-2\% isoflurane, placed 
in a 3-cm birdcage coil and scanned with a small-animal 9.4T MRI system (Bruker, Billerica, MA, USA). A contrast-enhanced T1-weighted 2D FLASH scan (TR/TE of $230 / 3.3 \mathrm{~ms}$, flip angle of $70^{\circ}$, number of excitations of 18, in-plane resolution of $85 \mu \mathrm{m} \times 85 \mu \mathrm{m}$, slice thickness of $500 \mu \mathrm{m}$ and receiver bandwidth of $50 \mathrm{kHz}$ ) was acquired $\sim 30-45$ min after FUS exposure, along both axial and coronal planes.

\subsubsection{Cavitation signal processing}

Time-domain signals recorded by the PCD were used to estimate the energy and cavitation dose for each pulsed-burst sonication during the entire procedure. First, a Fast Fourier Transform (FFT) of each recorded waveform was performed. Second, three spectral areas were filtered and analyzed independently. The first region corresponds to the spectra around the $n$-th harmonic, given by the frequency bands $n f_{c}-\Delta f \leq f_{h} \leq$ $n f_{c}+\Delta f$, where $f_{c}=1.68 \mathrm{MHz}$ is the center frequency of the FUS transducer, $n=2,3, \ldots, 10$ is the harmonic number, and the narrow bandwidth around each harmonic component is set to $\Delta f=10 \mathrm{kHz}$. The fundamental frequency is ignored due to the superposition of back-scattering and microbubble emission at this band. The second region corresponds to the spectra around the $n$-th ultra-harmonic, given by the frequency bands $(n+1 / 2) f_{c}-\Delta f \leq f_{u} \leq(n+1 / 2) f_{c}+\Delta f$. Note that the sub-harmonic $\left(f_{c} / 2\right)$ was not included in the calculation due to the limited bandwidth of the PCD. And the third spectral area corresponds to the broadband regions, given by the frequency band $f_{b}$, with $f_{h, n}+\Delta f<f_{b}<f_{u, n}-\Delta f$ and $f_{u, n}+\Delta f<f_{b}<f_{h, n+1}-\Delta f$. Third, cavitation doses were calculated as described before [7,38], based on the root-mean-square voltage detected in the respective spectral areas. Harmonic stable $\left(\mathrm{SCD}_{\mathrm{h}}\right)$, ultraharmonic stable $\left(\mathrm{SCD}_{\mathrm{u}}\right)$ and inertial (ICD) cavitation doses were defined as

$$
\mathrm{CD}_{i}=\frac{1}{N-1} \sum_{n=2}^{N} \sqrt{\left\langle|\mathcal{F}(V)|_{f_{i}}^{2}\right\rangle_{n}},
$$

where the index $i$ indicates harmonic $(h)$, ultra-harmonic $(u)$, and broadband regions $(b)$, to estimate $\mathrm{SCD}_{\mathrm{h}}, \mathrm{SCD}_{\mathrm{u}}$, and $\mathrm{ICD}$, respectively, $\mathcal{F}(V)$ is the fast Fourier transform of the voltage signals $V$, and $\langle\cdot\rangle$ is the average 
over the bands given by $f_{i} . \quad N=10$ bands were included. These doses were calculated for each acoustic pulse for in vivo experiments.

\subsubsection{BBB opening volume quantification}

Quantification was performed on the axial MRI slices for the $n=3$ mice. First, the region of interest (ROI) was defined in the front left part of the brain to calculate the reference intensity for each mice and hemisphere. The threshold intensity to define BBB opening was set as the average intensity within the control ROI plus 3 standard deviations. Every axial slice was loaded sequentially, and a manual ROI was drawn within the entire hemisphere. All pixels with intensity higher than the threshold were counted to derive the BBB opening surface area in each slice. The total $\mathrm{BBB}$ opening volume (BBBOV, in $\mathrm{mm}^{3}$ ) per mouse and hemisphere was calculated by summing the BBB opening surface areas across all hemisphere slices and then multiplying by the slice thickness. Finally, the contrast enhancement (CE, in \%) was calculated by dividing the mean intensity within the BBB opening areas by the mean intensity of the control ROI. To statistically compare the BBBOV and CE between right and left brain hemispheres, we used the Wilcoxon rank-sum test [39, 40], where statistical significance was assumed at $p<0.05$. BBBOV and CE results are presented as mean \pm standard deviation, unless otherwise stated.

\subsubsection{Acoustic calibration setup}

A calibration lens was designed and manufactured to test the splitting of the single focus into two foci in a water tank, and to assess the input electrical signal parameters needed to reach $400 \mathrm{kPa}$ of peak-negative pressure (PNP) at the focal spots. The calibration setup is shown in Fig. 3.2. Acoustic pressure-field measurements were conducted using an Onda HGL-0200 hydrophone (Sensitivity of $0.19 \mathrm{mV} / \mathrm{kPa}$ at $1.68 \mathrm{MHz}$ ) placed at the bottom of a degassed distilled water tank in a fixed position using a mechanical arm. A custom coupling cone was designed and 3D-printed to fix the lens to the transducer and to hold the coupling water. The entire system was attached to a computer-controlled, three-dimensional positioning system (Velmex Inc., Lachine, QC, CAN). The FUS transducer was driven by a function generator (Agilent, Palo 


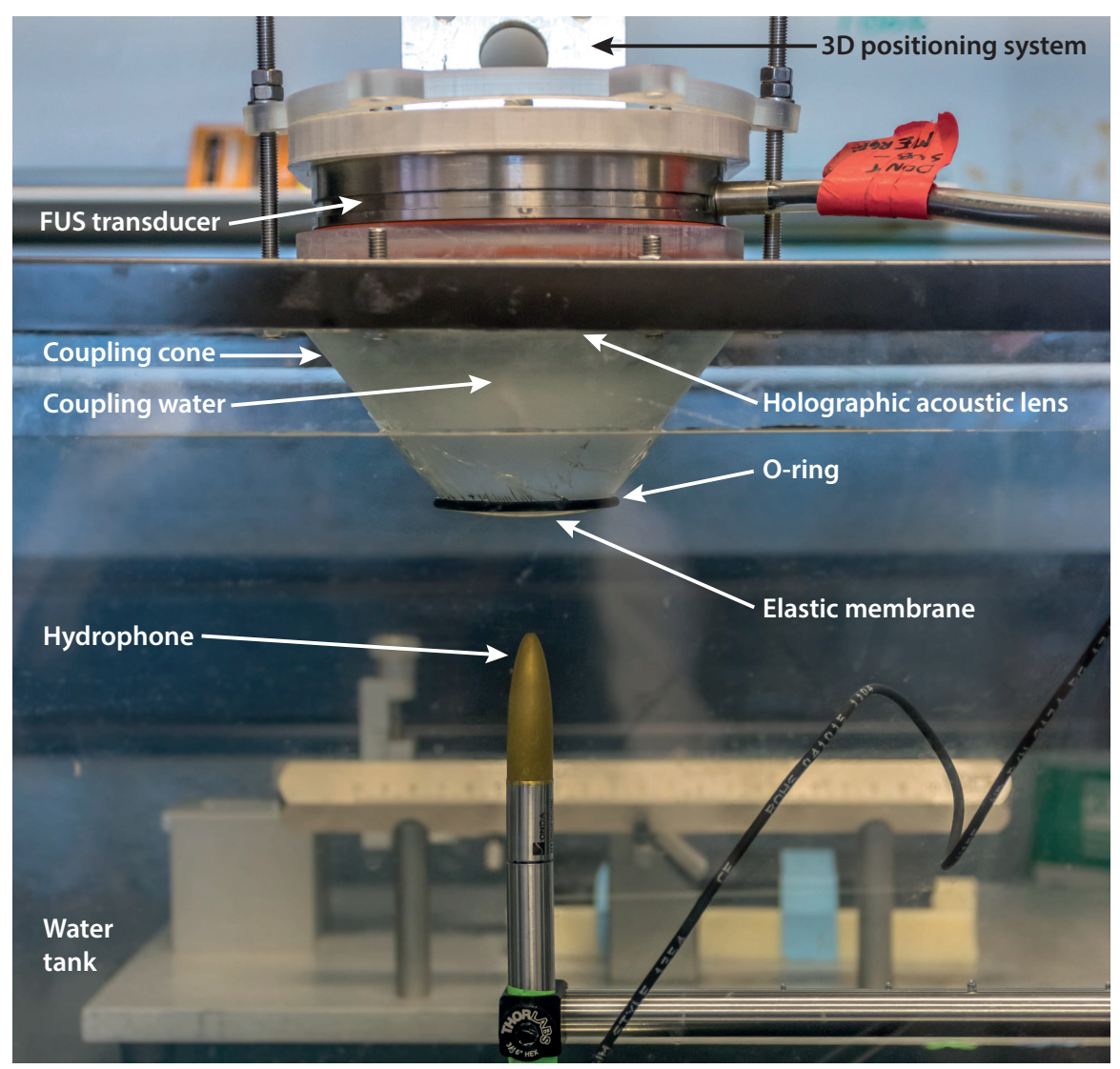

Figure 3.2: Calibration setup for the acoustic pressure-field measurements using the bifocal acoustic holographic lens in the water tank.

Alto, CA, USA) and a 50-dB power amplifier (325LA, ENI Inc., Rochester, NY, USA). For the $x-y$ planes, the field was scanned from $-5.3 \mathrm{~mm}$ to $5.3 \mathrm{~mm}$ in the $x$-direction and $-4.85 \mathrm{~mm}$ to $4.85 \mathrm{~mm}$ in the $y$-direction using a resolution of $0.1 \mathrm{~mm}$ ( 9 points per wavelength). For the $x-z$ planes, the field was measured from $-5.3 \mathrm{~mm}$ to $5.3 \mathrm{~mm}$ in the $x$-direction and $85 \mathrm{~mm}$ to $110 \mathrm{~mm}$ in the $z$-direction using a resolution of $0.176 \mathrm{~mm}$ ( 5 points per wavelength). 


\section{$3.3 \quad$ Results}

The results of this work are divided in two stages. First, we performed a calibration of the system designing a bifocal hologram in water. The pressure-field distribution was experimentally measured and compared to simulations. In this way, we assessed the accuracy of the method without biological tissues and we obtained the input electrical signal parameters to achieve $400 \mathrm{kPa}$ at the two foci in water. Second, we designed a new lens to compensate for the aberrations due to the coupling cone, skull and brain. We simulated the sonication through the head of the mouse to assess the focusing quality and estimate the attenuation of the ultrasound beam. Thus, by amplifying the input electrical signal accordingly, we proceeded to the sonication of the anesthetized animal and, finally, the BBB opening was evaluated using T1-weighted magnetic resonance imaging.

\subsubsection{System calibration}

A bifocal hologram was firstly designed for calibration in water. This lens splits the single focus of the FUS transducer into two foci, in addition to shifting the focal distance from $61 \mathrm{~mm}$ to $100 \mathrm{~mm}$. Fig. 3.3 and Fig. 3.4 show the simulated pressure-field distribution compared to the acoustic measurements in the water tank. The results are shown as PNP normalized to $p_{\mathrm{PNP}}$, the minimum pressure detected around the focal region. The elements forming the setup, i.e., the FUS transducer, lens, and coupling cone, are shown in Fig. 3.3 (a) as continuous-white line contours. The energy is concentrated on the focal distance of $100 \mathrm{~mm}$, where two spots are visible in the simulations and measurements. Experimental axial pressure-field distribution is in agreement with simulation (see Fig. 3.3 (a), insets). Experimental transverse patterns, shown in Fig. 3.3 (c), also are in agreement with numerical predictions, shown in Fig. 3.3 (b).

Taking a closer look at the pressure-field distribution, the cross-section of the acoustic field measured at $x=0 \mathrm{~mm}$ and $y=2.6 \mathrm{~mm}$, i.e., axially along the right focus, is shown in Fig. 3.4 (a). The maximum of the PNP is observed at $z=98.8 \mathrm{~mm}( \pm 0.3 \mathrm{~mm}$ of measurement resolution) in the experiments, compared to $z=100.2 \mathrm{~mm}$ in simulation $( \pm 0.18 \mathrm{~mm}$ of grid resolution). The experimental focal spot shows a depth-of-field of $19.2 \mathrm{~mm}$, about $4 \mathrm{~mm}$ longer than the $15 \mathrm{~mm}$ of the simulated field. The 

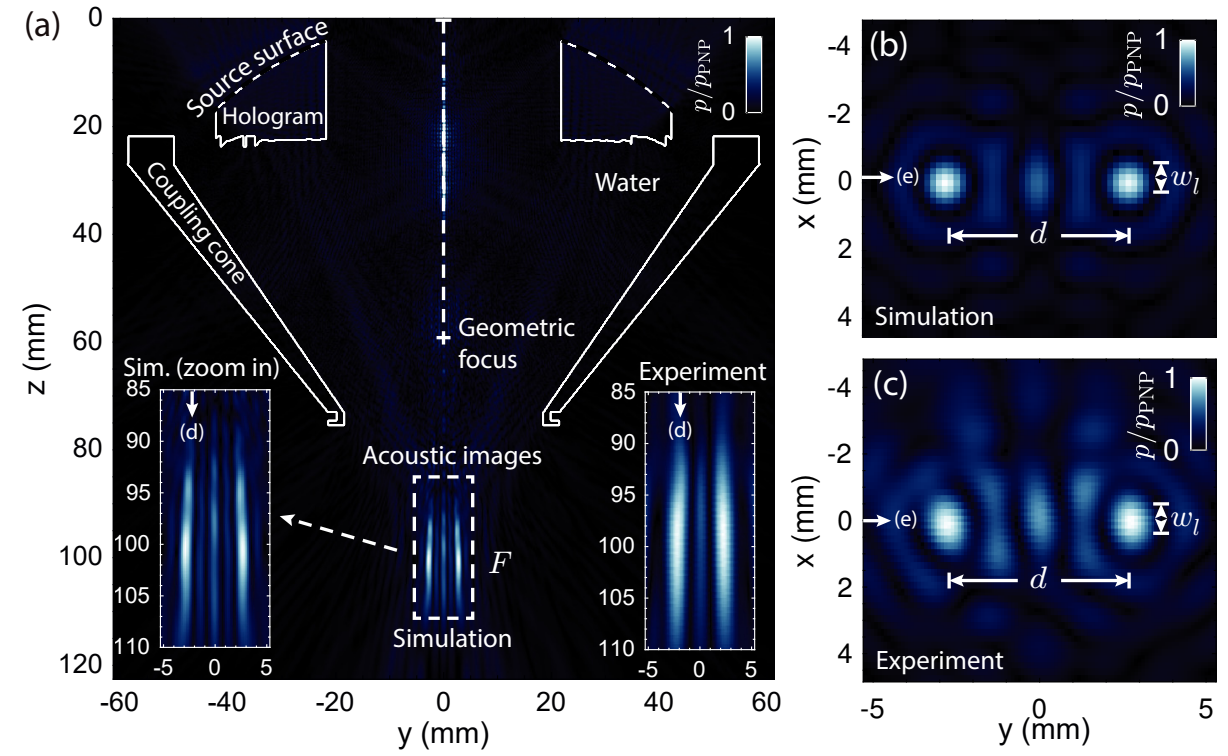

Figure 3.3: Peak-negative pressure-field distribution for the calibration of the holographic acoustic lens. (a) Simulated axial plane at $x=0 \mathrm{~mm}$ showing in the insets both a zoom of the simulated field and the experimental measurement at the focal region. The contours of the lens and cone are shown in a continuous-white line. (b,c) Simulated and experimental transversal planes at $z=100 \mathrm{~mm}$, respectively.

overall amplitude envelope follows the shape of the simulated field, even far away from the focal spot. Finally, Fig. 3.4 (b) shows the transversal cross-section at $z=100 \mathrm{~mm}$ and $x=0 \mathrm{~mm}$, i.e., transversely to both foci. The two symmetric focal spots appear at $y=-2.6 \mathrm{~mm}$ and $y=2.6 \mathrm{~mm}$ ( $\pm 0.18 \mathrm{~mm}$ numerically, $\pm 0.1 \mathrm{~mm}$ experimentally), respectively, in both simulation and experimental calibration. The full-width at half-maximum (FWHM) in the transverse direction is $1.10 \mathrm{~mm}( \pm 0.18 \mathrm{~mm}$ of grid resolution) along both $x$ and $y$ directions for the simulation, compared to $1.1 \mathrm{~mm}$ ( $\pm 0.1 \mathrm{~mm}$ of measurement resolution) along $y$-direction and $1.6 \mathrm{~mm}$ ( $\pm 0.1 \mathrm{~mm}$ of measurement resolution) along $x$-direction for the calibration. In addition, the experimental result shows a slightly higher 

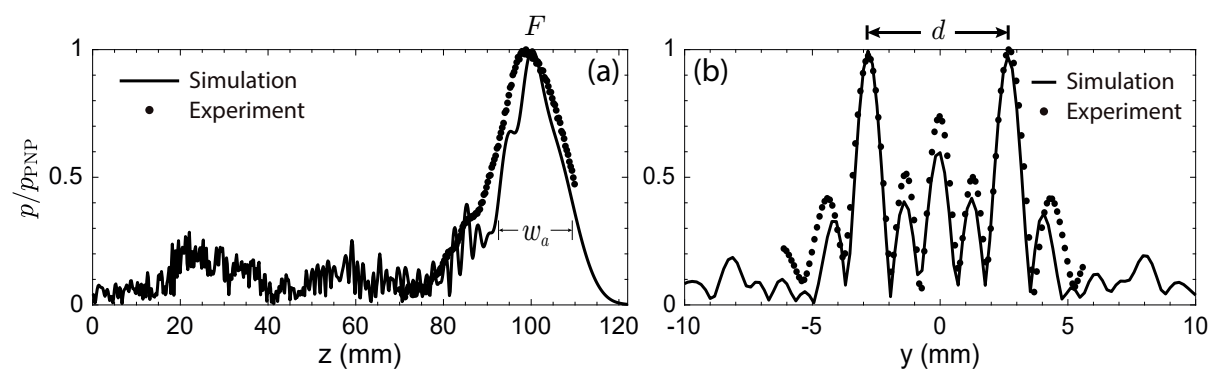

Figure 3.4: Cross-sections of the peak-negative pressure-field distribution for the calibration of the holographic acoustic lens. (a) Axial cross-section of the simulated (continuous-black line) and experimental (dotted-black line) pressure-field at $y=2.6 \mathrm{~mm}$ and $x=0 \mathrm{~mm}$. (b) Transversal cross-section of the simulated (continuous-red line) and experimental (dotted-black line) pressure-field at $z=100 \mathrm{~mm}$ and $x=0 \mathrm{~mm}$.

amplitude at secondary lobes, located at $y \approx-4.5,-1.5,1.5$ and $4.5 \mathrm{~mm}$, as shown in Fig. 3.4 (b). The experimental lobes show a mean normalized amplitude of 0.46 (0.38 in simulation) with a root-mean-square error of $\epsilon_{\mathrm{RMSE}}=0.087$. We observe another secondary lobe at $y=0 \mathrm{~mm}$ with a normalized amplitude of 0.74 in the experiment (0.60 in simulation, $\epsilon_{\mathrm{RMSE}}=0.087$ ), emerging due to the mirror symmetry of the hologram. Overall, the bilateral focal spots and secondary lobes obtained numerically agree the measured ones, thus proving the high accuracy of the holographic approach.

Then, the hydrophone was positioned at the center of one focus $(x=$ $0 \mathrm{~mm}, y=-2.6 \mathrm{~mm}$ and $z=98.8 \mathrm{~mm}$ ), and we performed acoustic measurements by changing the output voltage of the function generator, from $20 \mathrm{mV}$ to $280 \mathrm{mV}$ (peak) in steps of $20 \mathrm{mV}$. Using this data, we estimated the output voltage amplitude of the function generator needed to ensure $400 \mathrm{kPa}$ of PNP at the focus in water. To produce a PNP of $400 \mathrm{kPa}$ in water at the center of the foci, we calculated that the output voltage is $190 \mathrm{mV}( \pm 20 \mathrm{mV})$. Note that this value was enhanced at the treatment stage to compensate for the attenuation produced by the head of the mouse and the efficiency of the transcranial hologram, as explained below. 


\subsubsection{Bilateral BBB opening}

Once the lens was successfully calibrated in water, a transcranial simulation was performed to design a new lens that would take into account the aberrations of the skull and the brain of the mouse, in addition to the effects due to the coupling cone. This transcranial simulation initially indicated a pressure loss at the focus of $\approx 36 \%$ due to the attenuation of the skull and brain of the mouse, and due to the efficiency of the new hologram. The output voltage of the function generator was enhanced accordingly to $297 \mathrm{mV}$ to obtain a $400 \mathrm{kPa}-\mathrm{PNP}$ to open the BBB safely [41-44]. Two test mice were sonicated using these input parameters, and we detected gadolinium perfusion in T1-MRI images throughout almost the whole brain. The output voltage of the function generator was then reduced to $280 \mathrm{mV}$ ( $\approx 32 \%$ of water calibration), leading to a sharp bilateral BBB opening, indicating that the initial attenuation value was slightly overestimated by the simulation, including the rodent tissues, by a factor of $\approx 4 \%$. Three mice were treated at the chosen parameters to investigate feasibility across multiple animals.

A comparison between the simulated PNP distribution inside the head of the mouse, and the resulting BBB openings analyzing the post-sonication T1-MRI acquisitions, is shown in Fig. 3.5 for one of the mice. First, Fig. 3.5 (a) shows the simulated coronal pressure-field distribution at $x=$ $0 \mathrm{~mm}$ where continuous-white lines show the contours of the surface of the transducer, the hologram, the coupling cone, and the mouse skull. The pressure-field distribution shown here is normalized to $p_{\mathrm{PNP}}$, representing the PNP at the focal region inside the brain. A closer view of the coronal pressure-field distribution at the focal region is shown in Fig. 3.5 (c1), where the two symmetrical focal spots are marked by the two red arrows. The transverse (axial) cross-section of the simulated acoustic field at $z=$ $100 \mathrm{~mm}$ is shown in Fig. 3.5 (b1), where two clear circular spots are observed, as indicated by red arrows.

In vivo tests were performed as described in Methods section on $n=$ 3 mice. T1-MRI showed gadolinium extravasation at two symmetric focal spots in the axial and coronal planes, as shown in Figs. 3.5 (b2,c2), respectively. A bilateral $\mathrm{BBB}$ opening was achieved, as indicated by the two red arrows.

On the one hand, we can observe that the two experimental BBB 

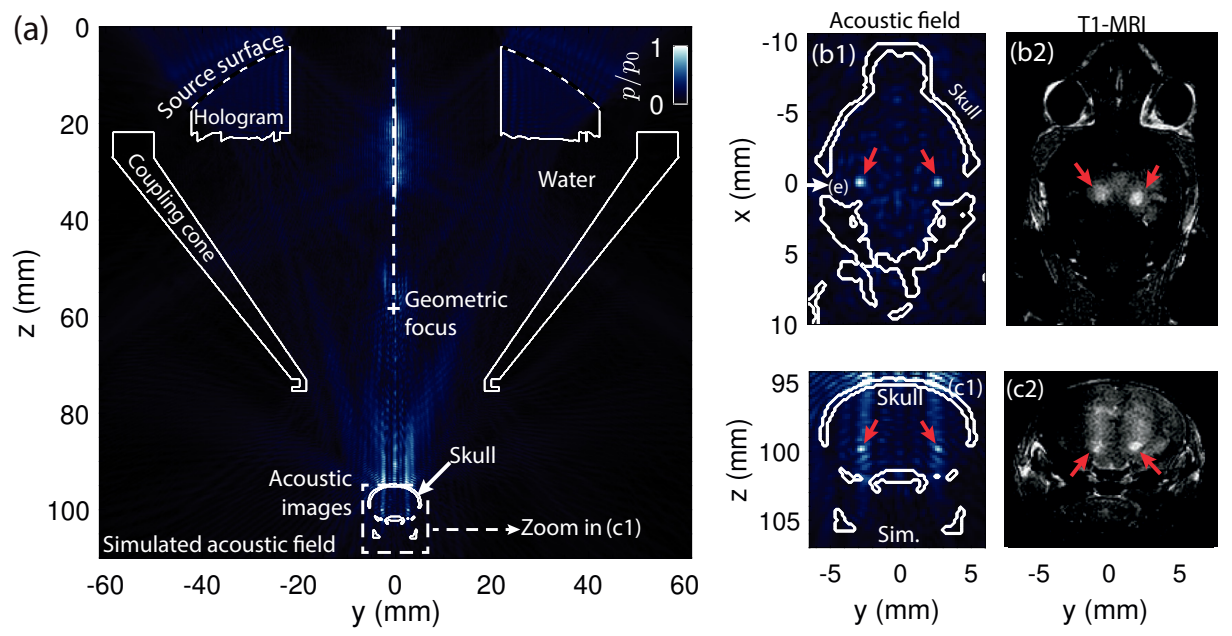

Figure 3.5: Peak-negative pressure-field distribution simulation compared to the BBB opening regions. (a) Simulated axial pressure-field distribution plane at $x=0 \mathrm{~mm}$. The contours of the lens, cone and skull are shown in a continuous-white line. (b1,c1) Simulated transversal (at $z=100 \mathrm{~mm}$ ) and axial (zoom in of (a) at $x=0 \mathrm{~mm}$ ) pressure-field distribution planes, respectively, showing the two focused spots by red arrows. (b2,c2) Experimental transversal and axial T1-MRI planes, respectively, showing the two BBB openings, in light color, by red arrows.

openings are separated $3.2 \pm 1.0 \mathrm{~mm}$ ( $n=3$ mice) compared to $5.3 \mathrm{~mm}$ in simulation. The discrepancy is likely due to the differences between the skull scanned with $\mu \mathrm{CT}$ for manufacturing the lens and the skulls of the treated mice or small misalignment of the system. Using the $\mu \mathrm{CT}$ scan of the treated subjects would lead to a better ultrasound location prediction for each mouse. Furthermore, other effects as imperfect coupling or small air pockets trapped within the lens surface may have contributed to the field distortion.

Average BBBOV was significantly different comparing both left and right hemispheres ( $p=0.01, n=3$ mice). The average BBBOV calculated was $17.7 \pm 6.8 \mathrm{~mm}^{3}$ and $34.9 \pm 9.6 \mathrm{~mm}^{3}$ for the left and right hemispheres, respectively. The right hemisphere showed a higher BBBOV, as observed in Fig. 3.5 (b2,c2), where the two BBB opening spots appear slightly 
displaced to the right hemisphere, observing how the right BBB opening spot is brighter than the left one. Consequently, off-target regions are also slightly brighter. This can be caused due to a misalignment of the transducer with respect to the mouse skull. Nevertheless, in CE, the average value was marginally different between hemispheres $(p=0.04$, $n=3$ mice). The calculated CE was $17.6 \pm 3.8 \%$ and $25.3 \pm 7.3 \%$ for the left and right hemispheres, respectively.

On the other hand, acoustic holograms showed a particular advantage when reconstructing an acoustic image inside the skull: the depth of field, i.e., the size of the focal spot in the $z$-direction, can be reduced compared to a progressive focused beam. This is clearly observed in the numerical results [see Fig. 3.6 (a)], where the field along the $z$-direction is shown for the hologram designed for the mouse and the one designed for water. Evaluating the axial cross-section, the depth of field of the focal spot for the transcranial case [continuous-red line in Fig. 3.6 (a)] is $\approx 0.5 \mathrm{~mm}$ (the wavelength at $1.68 \mathrm{MHz}$ in brain tissue is $0.95 \mathrm{~mm}$ ) compared to the
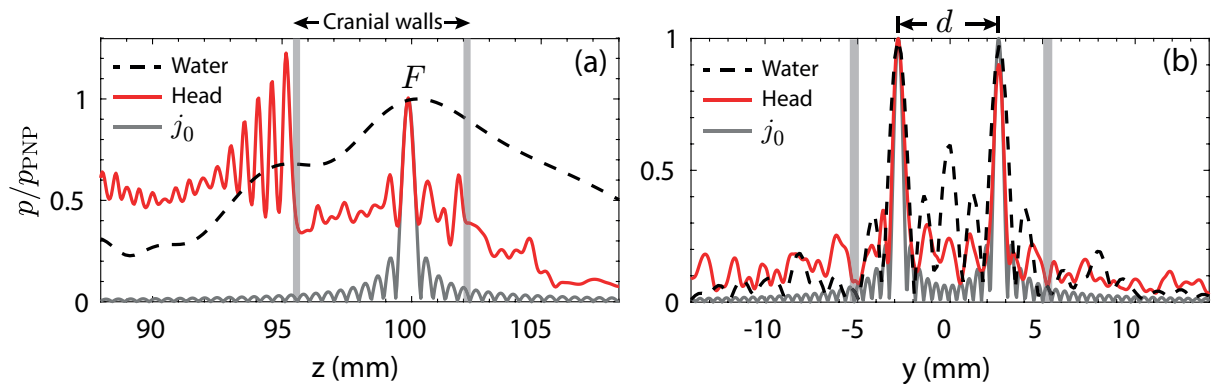

Figure 3.6: Cross-sections of the simulated peak-negative pressure-field distribution for calibration and transcranial configurations. (a) Simulated axial cross-section for the calibration case (dashed-black line) and for the transcranial case (continuous-red line) at $y=2.6 \mathrm{~mm}$ and $x=0 \mathrm{~mm}$. A spherical Bessel function of the first kind and zero-th order centered at the focal spot is shown in a continuous-gray line. (b) Simulated transversal cross-section for the calibration case (dashed-black line) and for the transcranial case (continuous-red line) at $z=100 \mathrm{~mm}$ and $x=0 \mathrm{~mm}$. A spherical Bessel function of the first kind and zero-th order centered at the two focal spots is shown in a continuous-gray line. 
$15 \mathrm{~mm}$ for the calibration case [dashed-black line in Fig. 3.6 (a)]. Furthermore, the full-width at half-maximum in the transversal direction is $\approx 0.6 \mathrm{~mm}$ for the transcranial case [continuous-red line in Fig. 3.6 (b)] compared to $\approx 1 \mathrm{~mm}$ for the calibration [dashed-black line in Fig. 3.6 (b)]. This enhancement of the focusing performance is associated with the ability of acoustic holograms to encode time-reversed wavefronts including the multiple reflections produced inside the mouse head, in analogy with other time-reversal systems [45-47]. Holograms encode waves in multiple directions by generating converging wavefronts to the focal spots and, simultaneously, secondary waves reflected on the walls of a leaky cavity, i.e., the skull and the walls of the coupling cone. Therefore, the angular spectrum of the resulting focused field by the hologram is enhanced compared to the angular spectrum of a progressive focused beam. In this way, using holograms for transcranial FUS, the focal spot that typically presents an elongated shape for a focused transducer converges to a spot of quasi-spherical shape. As a reference, the limiting case of a spherically converging wave, given by a spherical Bessel function of the first kind and zero-th order, $j_{0}(k r)$, where $k$ is the wavenumber in the brain at the source frequency and $r$ the radial distance centered at the focal spot, is plotted in Figs. $3.6(\mathrm{a}, \mathrm{b})$. We can see that the field produced by the hologram in the simulation is close to the theoretical diffraction limit, with the focal width being close to the half-wavelength limit. The corresponding focal spots are also visible in the experiments [see Figs. $3.5(\mathrm{~b} 2, \mathrm{c} 2)$ ], corroborating the bilateral BBB opening and the ability of holograms to improve the focusing capabilities of conventional transcranial FUS systems. They present a spherical shape, but as the gadolinium exhibits diffusion through the brain tissue, the experimental focal spots in the T1-weighted MRI are slightly bigger, showing a diameter size of $1.0 \pm 0.3 \mathrm{~mm}$.

\subsubsection{Cavitation analysis}

The FFT corresponding to a single therapeutic pulse showing the range of the harmonics $n=1,2, . ., 8$ is shown in Fig. 3.7 (a). The spectrogram for the whole experiment is represented in Fig. 3.7 (b), showing the stability on the microbubble emissions during the entire 2-min treatment, highlighting the prevalence of the harmonic frequencies $n=2,3,4$. At the top of Figs. $3.7(\mathrm{a}, \mathrm{b})$, we indicate the three analyzed spectral regions 
used to assess the cavitation doses. The total energy calculated for each pulsed-burst during the whole treatment is represented in Fig. 3.7 (c). The energy shows variations between pulses due to the different acoustic cavitation and the variations of microbubble concentration due to blood flow. However, the mean energy at the beginning and at the end of the treatment is similar. Harmonic stable $\left(\mathrm{SCD}_{\mathrm{h}}\right)$, ultraharmonic stable $\left(\mathrm{SCD}_{\mathrm{u}}\right)$, and inertial (ICD) cavitation doses are shown in Fig. 3.7 (d). First, $\mathrm{SCD}_{\mathrm{u}}$ remains high and constant during the whole procedure. In contrast, $\mathrm{SCD}_{\mathrm{u}}$ and ICD are two orders of magnitude lower than $\mathrm{SCD}_{\mathrm{u}}$, as it can also be visually identified in the spectrogram in Fig. 3.7 (b), confirming the absence of violent cavitation events which would compromise safety. In addition to the successful observed BBB opening shown previously, this fact indicates that cavitation activity was likely occurring during therapy in a stable and repeatable way.

\subsection{Conclusions}

In this work, we have demonstrated the first in vivo simultaneous multi-focal BBB opening in mice using a 3D-printed acoustic hologram. Holographic lenses were effectively designed using a simulation including the skull of a mouse obtained from a $\mu \mathrm{CT}$-scan, from where its heterogeneous properties were acquired. The manufactured acoustic hologram shows the ability to simultaneously focus at two mirrored targets in each hemisphere, allowing thus a cost- and time-effective procedure to open the blood-brain barrier of preclinical animal models. Furthermore, only one sonication and, consequently, one microbubble administration, were enough to open the BBB simultaneously at different brain regions. In this way, single-element systems based on acoustic holograms define an alternative technology to phased-array systems for this application.

Phased-array systems present several advantages, such as real-time steering. However, therapeutic phased-array systems are limited by the number of active elements, e.g., up to 2048. Typically, as a wide aperture is required for therapeutic phased arrays, their active piezoelectric elements are commonly separated by a distance greater than half-wavelength. Therefore, their steering capability is limited by the emergence of strong grating lobes. In contrast, acoustic holograms allow encoding wavefronts 

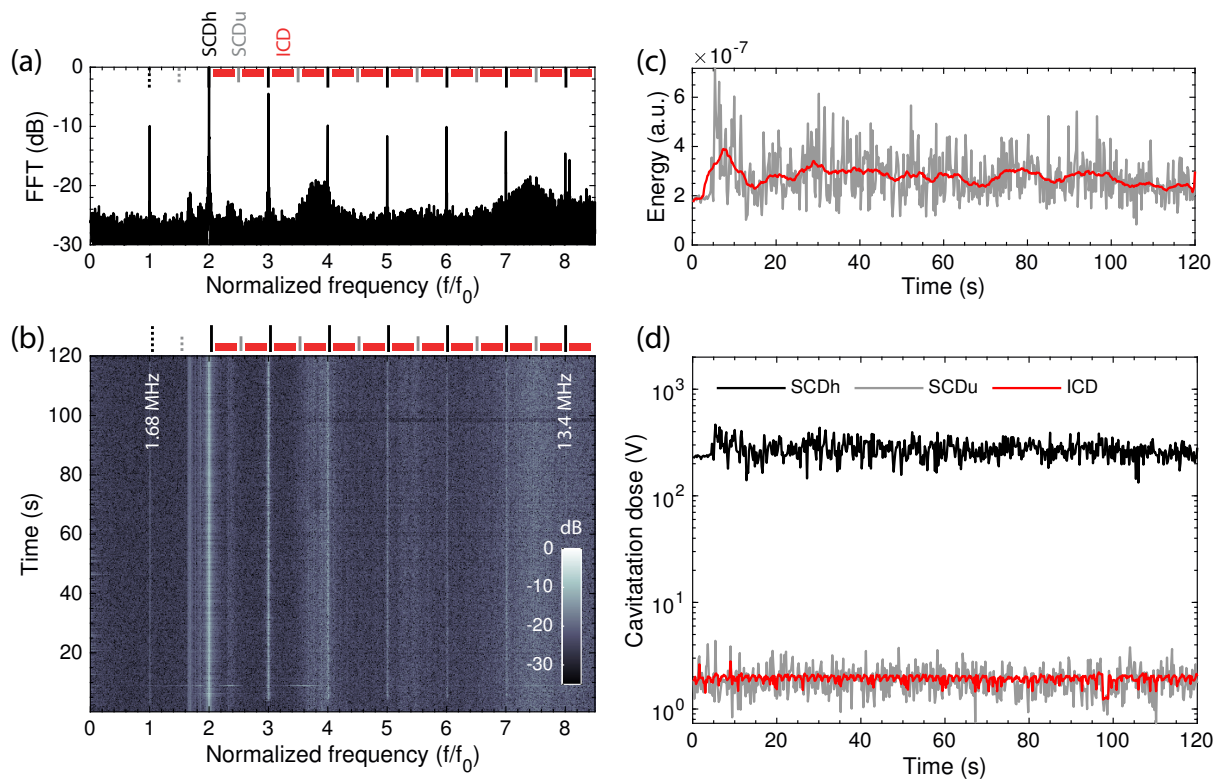

Figure 3.7: In vivo microbubble emission analysis over sonication time. (a) Normalized amplitude of the Fast Fourier Transform (FFT) performed over the cavitation emissions produced by a single pulse. (b) Spectrogram showing the evolution of the harmonics $n=1,2,3,4,5,6,7$. (c) Energy evolution during the entire treatment (gray) and energy using a 5-second moving average filter. (d) Stable harmonic (black), stable ultraharmonic (gray), and inertial (red) cavitation doses.

with a higher spatial resolution, in this work up to 131784 elements, resulting in an enhanced steering capability. In addition, these lenses allow synthesizing not only the progressive focused waves towards the targets, but also the multiple-scattered waves inside a leaky cavity of irregular shape such as the skull. The design process considers the time-reversed (or phase-conjugated) version of the back-propagated wave traveling from the target structure, through the aberrating media, towards the holographic plane [11]. Therefore, in addition to compensating for the phase aberrations introduced by the skull wall and the brain tissue, these acoustic lenses can replicate the reflections inside the head of the animal. This results in 
an enhancement of the angular spectrum of the synthesized waves, and, thus, the hologram can ideally reconstruct a sharp acoustic image close to the diffraction limit [Figs. $3.6(\mathrm{a}, \mathrm{b})]$. In practice, the depth-of-field of the focal spot, typically elongated for a focused ultrasound source, is greatly reduced in the axial direction. In this study, a quasi-spherical focal spot was obtained. Since the target structure is always enclosed in the cranial cavity for the treatment of brain diseases, holographic acoustic lenses show a great potential to enhance spatial resolution, becoming a safe, low-cost, and highly localized technique to target brain structures at the CNS in preclinical animal models.

The proposed approach presents several limitations which must be discussed and understood to control the technique accurately. On the one hand, the presented lenses are non-reconfigurable. Therefore, a personalized lens is ideally needed for each patient and treatment target. A new lens design is recommended to have an accurate beam control for each new focusing configuration, resulting in a time-inefficiency procedure for the same patient, due to removing and placing of the lenses for the different configurations. In this way, reconfigurable holograms, e.g., as those based on spatial sound modulators [16], are of great interest to transcranial ultrasound therapy. However, note that it has been previously proved that aberrations are still corrected despite a small misalignment, intentionally or not, in the transducer position [18]. This allows using the same lens and applying a small displacement to the transducer in order to move the focal spot, covering even a larger volume while still correcting the skull aberrations.

On the other hand, note that for clinical BBB opening applications in humans the central frequency (usually below $650 \mathrm{kHz}$ ) is lower than in preclinical applications (usually above $1 \mathrm{MHz}$ in mice). The thickness of the hologram, i.e., the height of each pixel, is proportional to the wavelength in the material of the lens. Therefore, holograms for preclinical applications require higher resolution 3D-printing techniques, and small printing errors could be detrimental in the focal shape generation.

This study shows the feasibility, capability, and efficiency of acoustic holograms for high resolution multi-focal blood-brain barrier opening. Moreover, the benefits of this approach can be widely useful in other applications such as neuromodulation, high-intensity focused ultrasound, 
ultrasound hyperthermia, transcranial imaging, or drug and gene delivery, defining a promising solution to the complex requirements of these biomedical ultrasound applications. 


\section{References - Chapter 3}

[1] R. Chopra, L. Curiel, R. Staruch, L. Morrison, and K. Hynynen, "An mricompatible system for focused ultrasound experiments in small animal models," Medical physics, vol. 36, no. 5, pp. 1867-1874, 2009.

[2] J. F. Jordão, C. A. Ayala-Grosso, K. Markham, Y. Huang, R. Chopra, J. McLaurin, K. Hynynen, and I. Aubert, "Antibodies targeted to the brain with image-guided focused ultrasound reduces amyloid- $\beta$ plaque load in the tgcrnd 8 mouse model of alzheimer's disease," PloS one, vol. 5, no. 5, p. e10549, 2010.

[3] S. Jalali, Y. Huang, D. J. Dumont, and K. Hynynen, "Focused ultrasoundmediated bbb disruption is associated with an increase in activation of akt: experimental study in rats," BMC neurology, vol. 10, no. 1, pp. 1-10, 2010.

[4] J. F. Jordão, E. Thévenot, K. Markham-Coultes, T. Scarcelli, Y.-Q. Weng, K. Xhima, M. O'Reilly, Y. Huang, J. McLaurin, K. Hynynen, et al., "Amyloid- $\beta$ plaque reduction, endogenous antibody delivery and glial activation by brain-targeted, transcranial focused ultrasound," Experimental neurology, vol. 248, pp. 16-29, 2013.

[5] T. Scarcelli, J. F. Jordão, M. A. O'reilly, N. Ellens, K. Hynynen, and I. Aubert, "Stimulation of hippocampal neurogenesis by transcranial focused ultrasound and microbubbles in adult mice," Brain stimulation, vol. 7, no. 2, pp. 304-307, 2014.

[6] M. E. Poorman, V. L. Chaplin, K. Wilkens, M. D. Dockery, T. D. Giorgio, W. A. Grissom, and C. F. Caskey, "Open-source, small-animal magnetic resonance-guided focused ultrasound system," Journal of therapeutic ultrasound, vol. 4, no. 1, p. 22, 2016.

[7] A. N. Pouliopoulos, S.-Y. Wu, M. T. Burgess, M. E. Karakatsani, H. A. Kamimura, and E. E. Konofagou, "A clinical system for non-invasive bloodbrain barrier opening using a neuronavigation-guided single-element focused ultrasound transducer," Ultrasound in medicine $\&$ biology, vol. 46, no. 1, pp. $73-89,2020$.

[8] N. Lipsman, Y. Meng, A. J. Bethune, Y. Huang, B. Lam, M. Masellis, N. Herrmann, C. Heyn, I. Aubert, A. Boutet, et al., "Blood-brain barrier opening in alzheimer's disease using mr-guided focused ultrasound," Nature communications, vol. 9, no. 1, pp. 1-8, 2018. 
[9] A. Abrahao, Y. Meng, M. Llinas, Y. Huang, C. Hamani, T. Mainprize, I. Aubert, C. Heyn, S. E. Black, K. Hynynen, et al., "First-in-human trial of blood-brain barrier opening in amyotrophic lateral sclerosis using mr-guided focused ultrasound," Nature communications, vol. 10, no. 1, pp. 1-9, 2019.

[10] M. Pernot, J.-F. Aubry, M. Tanter, J.-L. Thomas, and M. Fink, "High power transcranial beam steering for ultrasonic brain therapy," Physics in Medicine \& Biology, vol. 48, no. 16, p. 2577, 2003.

[11] S. Jiménez-Gambín, N. Jiménez, J. M. Benlloch, and F. Camarena, "Holograms to focus arbitrary ultrasonic fields through the skull," Physical Review Applied, vol. 12, no. 1, p. 014016, 2019.

[12] K. Melde, A. G. Mark, T. Qiu, and P. Fischer, "Holograms for acoustics," Nature, vol. 537, no. 7621, p. 518, 2016.

[13] Y. Xie, C. Shen, W. Wang, J. Li, D. Suo, B.-I. Popa, Y. Jing, and S. A. Cummer, "Acoustic holographic rendering with two-dimensional metamaterialbased passive phased array," Scientific reports, vol. 6, p. 35437, 2016.

[14] M. Brown, D. Nikitichev, B. Treeby, and B. Cox, "Generating arbitrary ultrasound fields with tailored optoacoustic surface profiles," Applied Physics Letters, vol. 110, no. 9, p. 094102, 2017.

[15] Y. Zhu, J. Hu, X. Fan, J. Yang, B. Liang, X. Zhu, and J. Cheng, "Fine manipulation of sound via lossy metamaterials with independent and arbitrary reflection amplitude and phase," Nature communications, vol. 9, no. 1, pp. 1-9, 2018.

[16] Z. Ma, K. Melde, A. G. Athanassiadis, M. Schau, H. Richter, T. Qiu, and P. Fischer, "Spatial ultrasound modulation by digitally controlling microbubble arrays," Nature communications, vol. 11, no. 1, pp. 1-7, 2020.

[17] J. Wang, F. Allein, N. Boechler, J. Friend, and O. Vazquez-Mena, "Design and fabrication of negative-refractive-index metamaterial unit cells for nearmegahertz enhanced acoustic transmission in biomedical ultrasound applications," Physical Review Applied, vol. 15, no. 2, p. 024025, 2021.

[18] G. Maimbourg, A. Houdouin, T. Deffieux, M. Tanter, and J.-F. Aubry, "3dprinted adaptive acoustic lens as a disruptive technology for transcranial ultrasound therapy using single-element transducers," Physics in Medicine E Biology, vol. 63, no. 2, p. 025026, 2018. 
[19] G. Maimbourg, A. Houdouin, T. Deffieux, M. Tanter, and J.-F. Aubry, "Steering capabilities of an acoustic lens for transcranial therapy: numerical and experimental studies," IEEE Transactions on Biomedical Engineering, vol. 67, no. 1, pp. 27-37, 2019.

[20] M. D. Brown, B. T. Cox, and B. E. Treeby, "Design of multi-frequency acoustic kinoforms," Applied Physics Letters, vol. 111, no. 24, p. 244101, 2017.

[21] M. D. Brown, "Phase and amplitude modulation with acoustic holograms," Applied Physics Letters, vol. 115, no. 5, p. 053701, 2019.

[22] M. D. Brown, B. T. Cox, and B. E. Treeby, "Stackable acoustic holograms," Applied Physics Letters, vol. 116, no. 26, p. 261901, 2020.

[23] S. Jiménez-Gambín, N. Jiménez, J. M. Benlloch, and F. Camarena, "Generating bessel beams with broad depth-of-field by using phase-only acoustic holograms," Scientific reports, vol. 9, no. 1, pp. 1-13, 2019.

[24] S. Jiménez-Gambín, N. Jiménez, and F. Camarena, "Transcranial focusing of ultrasonic vortices by acoustic holograms," Physical Review Applied, vol. 14, no. 5, p. 054070, 2020.

[25] J. Kim, S. Kasoji, P. G. Durham, and P. A. Dayton, "Acoustic holograms for directing arbitrary cavitation patterns," Applied Physics Letters, vol. 118, no. 5, p. 051902, 2021.

[26] M. Ferri, J. M. Bravo, J. Redondo, and J. V. Sánchez-Pérez, "Enhanced numerical method for the design of 3-d-printed holographic acoustic lenses for aberration correction of single-element transcranial focused ultrasound," Ultrasound in medicine 85 biology, vol. 45, no. 3, pp. 867-884, 2019.

[27] J.-F. Aubry, M. Tanter, M. Pernot, J.-L. Thomas, and M. Fink, "Experimental demonstration of noninvasive transskull adaptive focusing based on prior computed tomography scans," The Journal of the Acoustical Society of America, vol. 113, no. 1, pp. 84-93, 2003.

[28] C. W. Connor, Simulation methods and tissue property models for noninvasive transcranial focused ultrasound surgery. $\mathrm{PhD}$ thesis, Massachusetts Institute of Technology, 2005.

[29] C. W. Connor, G. T. Clement, and K. Hynynen, "A unified model for the speed of sound in cranial bone based on genetic algorithm optimization," Physics in Medicine \& Biology, vol. 47, no. 22, p. 3925, 2002. 
[30] R. S. Cobbold, Foundations of biomedical ultrasound. Oxford University Press, 2006.

[31] P. A. Yushkevich, J. Piven, H. C. Hazlett, R. G. Smith, S. Ho, J. C. Gee, and G. Gerig, "User-guided 3d active contour segmentation of anatomical structures: significantly improved efficiency and reliability," Neuroimage, vol. 31, no. 3, pp. 1116-1128, 2006.

[32] J. Choi, M. Pernot, T. Brown, S. Small, and E. Konofagou, "Spatio-temporal analysis of molecular delivery through the blood-brain barrier using focused ultrasound," Physics in Medicine 6 Biology, vol. 52, no. 18, p. 5509, 2007.

[33] J. J. Choi, M. Pernot, S. A. Small, and E. E. Konofagou, "Noninvasive, transcranial and localized opening of the blood-brain barrier using focused ultrasound in mice," Ultrasound in medicine 86 biology, vol. 33, no. 1, pp. 95$104,2007$.

[34] J. J. Choi, S. Wang, T. R. Brown, S. A. Small, K. E. Duff, and E. E. Konofagou, "Noninvasive and transient blood-brain barrier opening in the hippocampus of alzheimer's double transgenic mice using focused ultrasound," Ultrasonic imaging, vol. 30, no. 3, pp. 189-200, 2008.

[35] J. J. Choi, J. A. Feshitan, B. Baseri, S. Wang, Y.-S. Tung, M. A. Borden, and E. E. Konofagou, "Microbubble-size dependence of focused ultrasoundinduced blood-brain barrier opening in mice in vivo," IEEE Transactions on Biomedical Engineering, vol. 57, no. 1, pp. 145-154, 2009.

[36] M. Fink, "Time reversal of ultrasonic fields. i. basic principles," IEEE transactions on ultrasonics, ferroelectrics, and frequency control, vol. 39, no. 5, pp. 555-566, 1992.

[37] A. N. Pouliopoulos, D. A. Jimenez, A. Frank, A. Robertson, L. Zhang, A. R. Kline-Schoder, V. Bhaskar, M. Harpale, E. Caso, N. Papapanou, et al., "Temporal stability of lipid-shelled microbubbles during acousticallymediated blood-brain barrier opening.," Front. Phys, vol. 8, p. 137, 2020.

[38] S.-Y. Wu, C. Aurup, C. S. Sanchez, J. Grondin, W. Zheng, H. Kamimura, V. P. Ferrera, and E. E. Konofagou, "Efficient blood-brain barrier opening in primates with neuronavigation-guided ultrasound and real-time acoustic mapping," Scientific reports, vol. 8, no. 1, pp. 1-11, 2018.

[39] M. Hollander, D. A. Wolfe, and E. Chicken, Nonparametric statistical methods, vol. 751. John Wiley \& Sons, 2013. 
[40] J. D. Gibbons and S. Chakraborti, Nonparametric statistical inference. CRC press, 2020.

[41] K. Hynynen, N. McDannold, N. A. Sheikov, F. A. Jolesz, and N. Vykhodtseva, "Local and reversible blood-brain barrier disruption by noninvasive focused ultrasound at frequencies suitable for trans-skull sonications," $\mathrm{Neu}$ roimage, vol. 24, no. 1, pp. 12-20, 2005.

[42] Y.-S. Tung, F. Vlachos, J. J. Choi, T. Deffieux, K. Selert, and E. E. Konofagou, "In vivo transcranial cavitation threshold detection during ultrasound-induced blood-brain barrier opening in mice," Physics in Medicine 8 Biology, vol. 55, no. 20, p. 6141, 2010.

[43] Y.-S. Tung, F. Vlachos, J. A. Feshitan, M. A. Borden, and E. E. Konofagou, "The mechanism of interaction between focused ultrasound and microbubbles in blood-brain barrier opening in mice," The Journal of the Acoustical Society of America, vol. 130, no. 5, pp. 3059-3067, 2011.

[44] M. A. Valdez, E. Fernandez, T. Matsunaga, R. P. Erickson, and T. P. Trouard, "Distribution and diffusion of macromolecule delivery to the brain via focused ultrasound using magnetic resonance and multispectral fluorescence imaging," Ultrasound in Medicine 6 Biology, vol. 46, no. 1, pp. 122$136,2020$.

[45] A. Derode, P. Roux, and M. Fink, "Robust acoustic time reversal with highorder multiple scattering," Physical review letters, vol. 75, no. 23, p. 4206, 1995.

[46] C. Draeger and M. Fink, "One-channel time reversal of elastic waves in a chaotic 2d-silicon cavity," Physical Review Letters, vol. 79, no. 3, p. 407, 1997.

[47] M. Fink, "Time-reversal waves and super resolution," in Journal of Physics: Conference Series, vol. 124, p. 012004, IOP Publishing, 2008.

[48] Cover image for this chapter, creator name: Jasongeorge, stock.adobe.com.

\section{Publications}

The contents of this chapter, and closely related ones, were presented in the following publications: 


\section{Journal papers}

- S. Jiménez-Gambín, N. Jiménez, A. N. Pouliopoulos, J. M. Benlloch, E. E. Konofagou, and F. Camarena, "Acoustic holograms for bilateral blood-brain barrier opening in a mouse model". In process of manuscript submission on May, 2021.

\section{Conference proceedings}

- R. Bailén, S. Jiménez-Gambín, M. Company, and F. Camarena, "Desarrollo de un sistema ultrasónico de potencia para aplicaciones médicas en animales pequeños". $48^{\circ}$ Congreso Español de Acústica; Encuentro Ibérico de Acústica; European Symposium on Underwater Acoustics Applications; European Symposium on Sustainable Building Acoustics; TECNIACUSTICA 2017, A Coruña, Galicia, Spain. October 4-6, 2017.

- S. Jiménez-Gambín, A. N. Pouliopoulos, N. Jiménez, J. M. Benlloch, E. E. Konofagou, and F. Camarena, "Bilateral blood-brain barrier opening in mice using acoustic holograms". 7th International Symposium on Focused Ultrasound, virtual symposium. November 8-13, 2020. Special mention: one of the three most watched oral presentations.

- S. Jiménez-Gambín, A. N. Pouliopoulos, N. Jiménez, J. M. Benlloch, E. E. Konofagou, and F. Camarena, "First in vivo demonstration of bilateral blood-brain barrier opening using acoustic holograms in mice". IEEE International Ultrasonics Symposium (IUS), virtual symposium. September 7-11, 2020.

- S. Jiménez-Gambín, A. N. Pouliopoulos, E. E. Konofagou, N. Jiménez, J. M. Benlloch, and F. Camarena, "First in-vivo demonstration of targeted drug delivery using acoustic holograms in mice". 51 Congreso Español de Acústica; TECNIACUSTICA 2020; XI Congreso Ibérico de Acústica, virtual congress. June 3-5, 2020. Award: Andrés Lara Prize, ex aequo award for young researchers in its eighteenth edition. 




\subsection{Introduction}

Pediatric population is the most affected by cancer caused by primary brain tumors [1]. Diffuse Intrinsic Pontine Glioma (DIPG), recently known as diffuse midline glioma (DMG) - H3K27M-mutant [2], is the most aggressive primary pediatric brain tumor. This tumor is typically very infiltrative, and it is located in the pons within the brainstem. To date, there is no cure nor effective treatments that provide a long survival period, and the median overall survival is less than 1 year with Radiation Therapy (RT). RT is the only treatment which offers temporary palliative control. Conversely, conventional treatments are based on maximal surgical resection followed by adjuvant radiotherapy and chemotherapy. They are limited in efficacy because of the infiltrative nature of these tumors. Moreover, it is not possible to perform a total resection because cancerous cells are still infiltrating surrounding healthy tissues. On the other hand, the $\mathrm{BBB}$ prevents most therapeutic drugs from crossing the brain parenchyma and they can not reach the target cancerous cells [3].

Different approaches have been assessed in previous works to treat primary brain tumors clinically. Some of them by disrupting the BBB, such as mannitol administration [4] and the use of the bradykinin agonist RMP-7 [5], and some others by direct injection of drugs into the brain [6-8]. However, these techniques have not been widely used due to difficulties related to their routine implementation in the clinic. An alternative approach is the use of focused ultrasound and microbubbles to safely, localized, transiently, and reversibly increase the permeability of the BBB, which has been successfully demonstrated in small and large animals and preclinical glioma models. However, the ultrasound beam propagation through the skull is complex and difficult to control accurately. The skull is the most relevant obstacle in ultrasound treatments for Alzheimer's, Parkinson's, amyotrophic lateral sclerosis, and even neuro-oncology. This irregular and thick bone layer distorts and attenuates the focused beam leading to undesired effects [9]. Conventional FUS systems show advantages and drawbacks, but none of them completely accomplishes this type of clinical procedure requirements in a time- and cost-efficient manner. The ideal approach would consist of a non-invasive and localized BBB disruption throughout the entire brain tumor, which usually spreads over 
large brain regions. A novel technology using acoustic holograms coupled to a single-element FUS transducer can solve these limitations.

In this chapter, we study the feasibility and capabilities of using acoustic holographic lenses [10], coupled to a $250 \mathrm{kHz}$ single-element FUS transducer [11], a new non-invasive approach to increase the targeted volume of a pediatric cancer tumor in the brain while reducing the off-target FUS delivery. These lenses allow to compensate and correct the non-desired effects generated by the skull, such as focus deviation and deformation, and to generate a focal shape matching the geometry of the tumor, thus improving the efficiency and the performance of the treatment in comparison with the procedure using the conventional single-element FUS transducer. Different lens designs were studied, and the results indicated that the larger tumor covering with a reduced off-target dispersion was obtained with the curved-slice-hologram configuration, where the single-element FUS transducer had an outer diameter aperture of $132 \mathrm{~mm}$, a radius of curvature of $110 \mathrm{~mm}$, and no central hole, providing coverage of the $15.9 \%$ of the target tumor.

\subsection{Materials and methods}

The developed numerical procedure consisted of four steps. First, we acquired the 3D geometry and acoustical properties of a pediatric skull from X-ray CT images, and we identified the target tumor structure (DMG - H3K27M-mutant) from MRI. Second, an FDA-approved single-element FUS transducer, working at a central frequency of $250 \mathrm{kHz}$, was chosen for this study. Third, the transducer was positioned pointing towards the tumor through the occipital/parietal skull area. Lastly, an acoustic lens was numerically designed from the phase information recorded at the holographic surface. Afterward, it was coupled to the transducer surface to finally simulate the pressure-field distribution inside the pediatric human head.

\subsubsection{Numerical simulation}

We selected a pseudo-spectral simulation method with $k$-space dispersion correction to numerically integrate the linearised constitutive relations of 
acoustics [see Sec. 2.2.2 as explained in Chapter 2]. We used a numerical grid with a spatial step of $\Delta x=\Delta y=\Delta z=1 \mathrm{~mm}$ and a numerical temporal step of $\Delta t=57.7 \mathrm{~ns}$, leading to a Courant-Friedrichs-Lewy number of 0.2 and a spatial sampling of 6 grid points per wavelength in water for a frequency of $250 \mathrm{kHz}$. These parameters were fixed in all simulations in this chapter.

\subsubsection{CT and MRI acquisition}

First, in order to model the skull geometry, we used a CT-scan from a human pediatric head with a slice thickness of $2 \mathrm{~mm}$ and in-slice pixel spacing of $1 \times 1 \mathrm{~mm}$ (interpolated to $1 \mathrm{~mm}$ isotropic for the numerical simulation, 6 points per smallest wavelength at $250 \mathrm{kHz}$ ) from Columbia University. We defined the heterogeneous sound speed and density maps of the skull using the procedure described in Chapter 2 [see Sec. 2.2.1] but working at $250 \mathrm{kHz}$. The absorption of the pediatric skull was set to $1.5 \mathrm{~dB} / \mathrm{cm}$ [12]. After, a specialized neurosurgeon segmented the tumor from the MRI using the ITK-SNAP software [13], with an original resolution of $0.4 \times 0.4 \times 4.4 \mathrm{~mm}$ interpolated to $1 \mathrm{~mm}$ isotropic for the simulation. This tumor represents a $2.7 \%$ of the whole brain $\left(V_{\text {tumor }}=31.1 \mathrm{~cm}^{3}\right.$ compared to $\left.V_{\text {brain }}=1132.4 \mathrm{~cm}^{3}\right)$. Acoustical properties of the human pediatric brain were also included in the simulations to obtain a more realistic result, using a sound speed of $c_{\text {brain }}=1560 \mathrm{~m} / \mathrm{s}$, a density of $\rho_{\text {brain }}=1000 \mathrm{~kg} / \mathrm{m}^{3}$, and absorption of $0.10 \mathrm{~dB} / \mathrm{cm}$ at $250 \mathrm{kHz}$ [12].

\subsubsection{Lens design}

The distribution of heights along the lens surface was numerically obtained from the complex pressure-field distribution recorded at the holographic surface (see Sec. 2.2.3 for more details). A set of virtual sources distributed along the tumor were used for the back-propagation step. The lens was made of a 3D-printable resin from the company Formlabs (ClearResin material with a sound speed of $c_{L}=2580 \mathrm{~m} / \mathrm{s}$, a density of $\rho_{L}=1171 \mathrm{~kg} / \mathrm{m}^{3}$, and absorption of $\alpha=0.69 \mathrm{~dB} / \mathrm{cm}$ at $250 \mathrm{kHz}$ ).

Initially, two lens designs were developed for the FDA-approved transducer. The first configuration allowed to reconstruct a bifocal acoustic image inside the tumor, and the second configuration allowed to pro- 
duce a slice of the tumor. For these two configurations, the holographic design surface was a plane in front of the transducer surface (as described in Chapter 2, Sec. 2.3 and Sec. 2.5, for flat-bifocal-hologram and flat-slice-hologram configurations, respectively). However, designing a lens from a flat holographic surface is not efficient when the transducer has a curved surface, because, on the one hand, the governing equations of the Fabry-Perot resonator state that the resonating column has to vibrate perpendicularly to the source surface. On the other hand, the resulting geometry of the lens, including its back part, would be too thick, and losses would increase. Therefore, in this chapter, we included a new lens design by recording the holographic wavefront at a curved and parallel surface to the transducer surface, thus facilitating and improving the longitudinal vibration mode of each column of the lens and as well reducing internal losses in the lens material. Furthermore, we tested how the acoustic image reconstruction quality improved when increasing the aperture of the transducer and also when the central hole for the PCD was not included.

\subsubsection{Quality parameters evaluation}

For each numerical case studied, three quality parameters were obtained to compare the different approaches. Previous to the quality parameters calculation, we defined the active ultrasound beam volume 3D mask where the delivered pressure remains in the safe range for BBB opening of $0.25-0.5 \mathrm{MPa}[11,14]$. This volume was defined at the decay of half the maximum pressure at the focus (from $P_{\max } / 2$ to $P_{\max }$ ) and was used to obtain the in-target and off-target volumes covered by the active ultrasound beam. On the one hand, we defined $V_{i n}$ as the volume of the tumor covered by the active beam. The higher $V_{i n}$ is, the higher the coverage of the tumor is, and the more efficient and localized the treatment is. On the other hand, we defined $V_{\text {out }}$ as the off-target volume (the volume of the brain that does not belong to the tumor) covered by the active beam. In this way, we assessed the pressure delivery, within the safe range for BBB opening, to the non-desired tumor-adjacent brain tissue. The smaller $V_{\text {out }}$ is, the less off-target coverage, and the more localized the treatment is.

The third quality parameter is related to the gain of the FUS device. The gain of a FUS system characterizes, on the one hand, its efficiency in generating a specific pressure at the focus for a given input signal, and on 
the other hand, how sharp the focus can be generated. In such a way, the higher the gain, the higher the focal pressure and the sharper the focus is generated, and vice-versa. Working with a sharp focus is beneficial for BBB opening FUS treatments, as a sharp and clear difference between the focal and non-focal pressure values is obtained. The skull attenuation prediction, which usually presents uncertainties, is relevant as well to estimate the pressure delivered at the target structure. Note that two pressure regions can be identified for BBB opening applications: the focal region where the $\mathrm{BBB}$ is expected to be opened, i.e., from $P_{\max } / 2$ to $P_{\max }$, and the non-focal or background region where no BBB opening is expected, i.e., from zero to $P_{\max } / 2$. Then, for a low-gain FUS system, the non-sharp focus would bring the background and focal regions closer to each other, facilitating that uncertainties in the skull attenuation prediction led to an excessive off-target BBB opening (overestimated attenuation), or on the contrary, led to a reduced in-target BBB opening (underestimated attenuation); even a non-existent BBB opening could be obtained if an excessive underestimation of the attenuation was assumed. On the contrary, for a high-gain FUS system, the sharp focus generated would allow making relatively small errors in the attenuation prediction, as the background region pressure values would still stay sharply away from the focal values. In this chapter, acoustic holograms allow for spreading the single focus of a single-element FUS transducer along the tumor, which leads to an implicit reduction in the focal pressure. Therefore, the gain of the FUS system (transducer and lens) is reduced even more. Although we can amplify the external input signal accordingly, this low-gain FUS system will produce an even less sharp focus, and skull attenuation uncertainties are critical for a successful BBB opening procedure. For the different approaches for FUS delivery in a pediatric brain tumor studied in this chapter, it is defined the quality parameter $R A R=A_{\text {lens }} / A_{\text {transducer }}$ (Relative focal Amplitude Reduction) to assess the reduction of the amplitude (pressure) at the maximum of the focus, $A_{\text {lens }}$, obtained transcranially using a lens, compared to the transcranial focal amplitude provided by the single-element transducer, $A_{\text {transducer }}$, both amplitudes obtained for the same amplification factor of the external input signal. This $R A R$ is partially related to the losses due to the propagation through the skull. However, it is mainly related to the active aperture of the transducer and to the spreading of 
the FUS focus along the target tumor. For the transcranial configuration without lens $R A R=1$, and with lens $0<R A R<1$. Thus, the smaller $R A R$ is, the more sensible and complicated it is to ensure $0.25-0.5 \mathrm{MPa}$ at the focal region with the FUS system against existing uncertainties in the skull attenuation prediction. For this reason, we must consider the focusing gain of the FUS system to assess the complexity of achieving successful treatment.

The interest in using an acoustic holographic lens lies in achieving a higher $V_{i n}$ than the conventional single-element FUS transducer while keeping a reduced $V_{\text {out }}$ not to spread the focus outside the tumor, finally ensuring a high $R A R$ for a simple-task and fast clinical procedure.

\subsubsection{Optimal holographic wavefront definition}

The target image reconstruction quality provided by these lenses, i.e. the capability of spreading the focus along the pediatric tumor, is limited if working at such a low frequency $(250 \mathrm{kHz})$ for such a reduced aperture of the FDA-approved transducer $(2 a=110 \mathrm{~mm})$, as shown later in results in Sec. 4.5 and discussed in Sec. 4.5.1. Due to this limitation, the target image reconstruction quality provided by these lenses is initially poor. It could be improved by using an iterative optimizing method to calculate the holographic plane, but this is prohibitive for 3D heterogeneous simulations of large domains such as a human skull [see Chapter 2, Sec. 2.2.3 for more details]. A second approach could be to increase both the aperture and working frequency of the transducer, to work with higher angular spectrum. However, if the geometry and working frequency of the transducer are already FDA-approved, i.e., fixed, there is an alternative that will allow us to slightly improve the resulting target image reconstruction quality: to add a constant phase offset to the recorded phase of the holographic design surface. What makes a holographic lens work properly is not the specific phase value at the output of each column, but the phase difference between each column and its neighbors. In other words, the relative phase differences between columns throughout the entire lens surface. Therefore, once the holographic surface information is recorded (using a single back-propagation iteration), we can then add a constant phase offset to all the columns $0<\phi_{\text {offset }}<2 \pi \mathrm{rad}$, while the relative phase differences between neighbor columns remain constant. 
Fig. 4.1 shows an example of this phase offset addition for one of the designed holographic lenses to create a broad focus targeting the cancer tumor. Once we do the backward propagation, we obtain the holographic phase surface shown in Fig. 4.1(a1). Afterward, we create the lens, and we do the forward propagation resulting in the pressure field distribution shown in Fig. 4.1(a2), where a broad focus is covering the tumor (in this particular case, the focus shows a void in its center because we are working with a small aperture compared to such a low frequency). Fig. 4.1(b1) shows the holographic phase surface when a phase offset of $\phi_{\text {offset }}=2 \pi / 3 \mathrm{rad}$ is added. As we can see, the relative phase differences along the whole holographic surface remain constant, but the singular phase values of the columns are different. Despite of adding this phase offset, the new lens still produces a very similar acoustic image, if we observe the pressure field distribution in Fig. 4.1(b2). Although an evident different shape of the lens (its contour is shown in continuous white line) is observed, we can just distinguish a slight difference in the pressure field distribution. Thus the new modified lens is still working similarly. A third example is shown in Fig. 4.1(c1,c2), where a phase offset of $\phi_{\text {offset }}=4 \pi / 3 \mathrm{rad}$ is added, and the pressure field distribution slightly changes. In all cases, the shape of the focused beam is almost the same, but if we take a closer look at the pressure at the edges of the tumor, or in terms of coverage between focal region and tumor, we can observe a variation in the tumor coverage (different $V_{i n}$ ) as a function of this added phase offset. Therefore, to assess the overall behavior of adding a phase offset between the entire range of 0-2 $\pi$ rad (a complete revolution), Fig. 4.1(d,e) shows the quality parameters $V_{\text {in }}, V_{\text {out }}$ and $R A R$, respectively, as a function of the added phase offset. We can see that, as $V_{\text {in }}$ increases, so does $V_{\text {out }}$, but there are values of $\phi_{\text {offset }}$ which provide a lower $V_{\text {out }}$ for a given $V_{\text {in }}$. On the contrary, around $\phi_{\text {of fset }}=1.15 \pi \mathrm{rad}$, where $V_{\text {in }}$ is maximum, the focal region is covering almost the same volume outside than inside the tumor. For this study, we choose $\phi_{\text {of } f s e t}$ by evaluating the minimum value provided by the optimization function $f_{\text {opt }}$ defined as:

$$
f_{\text {opt }}=V_{\text {out }}-V_{\text {in }}
$$

where $V_{\text {out }}$ and $V_{\text {in }}$ are the quality parameters representing the off-target and in-target covered volumes, respectively. The minimum value of this 

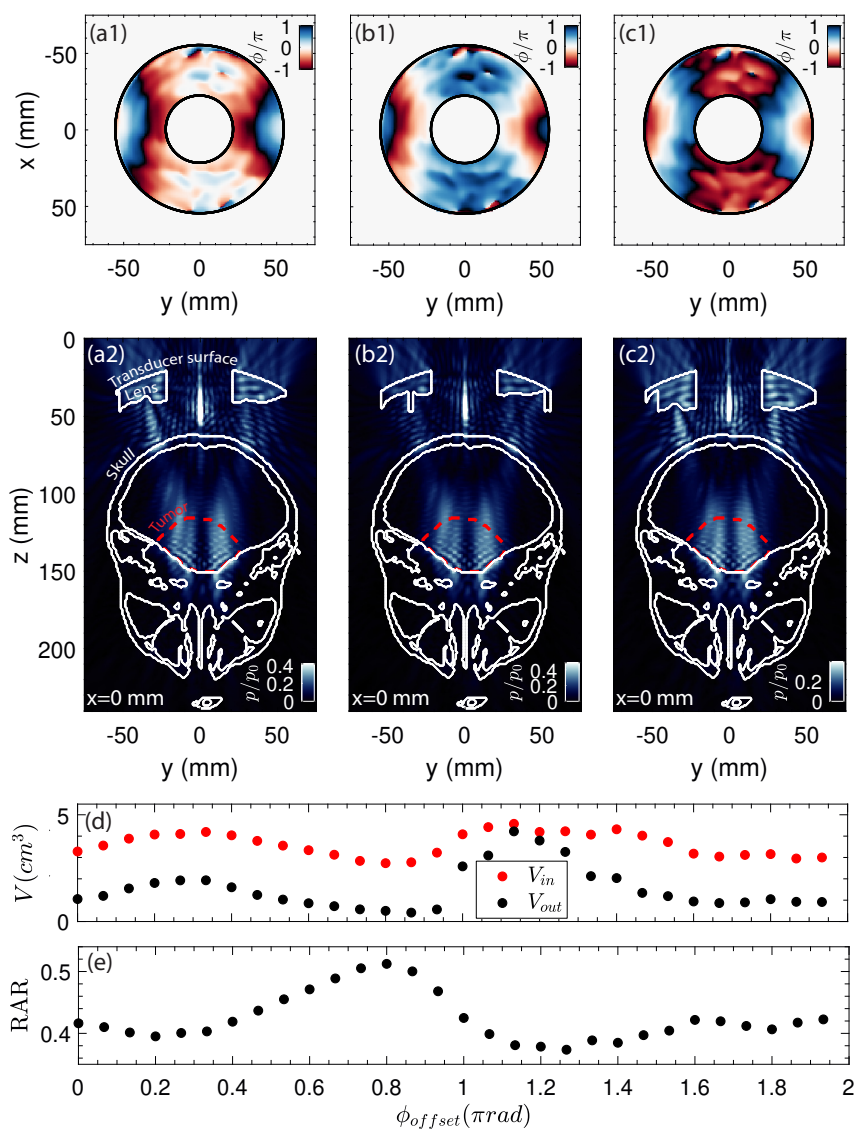

Figure 4.1: Holographic phase information and corresponding pressure field distribution as a function of the added constant phase offset. (a1,b1,c1) Phase distribution at the holographic plane when the phase offset is $\phi_{\text {offset }}=0,2 \pi / 3$ and $4 \pi / 3 \mathrm{rad}$, respectively. (a2,b2,c2) Axial cut of the pressure field distribution at $x=0 \mathrm{~mm}$ showing the contours of the transducer surface, lens and skull (continuous white line) and tumor (dashed red line) when the phase offset is $\phi_{\text {off set }}=0,2 \pi / 3$ and $4 \pi / 3 \mathrm{rad}$, respectively. (d) Overlapping quality parameters $V_{\text {in }}$ and $V_{\text {out }}$, and (e) gain-related quality parameter $R A R$ as a function of the added phase offset. 
optimization function corresponds to the optimal phase offset $\phi_{\text {off set,opt }}$ that will provide the highest $V_{\text {in }}$ for the lowest $V_{\text {out }}$. In this study, the aim is to cover a considerable and greater amount of tumor volume compared to the conventional single-element FUS system without a lens. In this way, we select a minimum value for $V_{i n}$ following the condition $V_{i n} \geq n V_{i n, T}$, where $V_{i n, T}$ is the in-target covered volume by only using the single-element FUS transducer, and $n$ is a positive real number greater than the unity. For the bifocal lens approach, $n=2$, while for the rest holographic approaches, $n=2.5$. Finally, and for all holographic approaches, the pressure-field distribution shown in the results is obtained with a lens designed by adding the optimal phase offset $\phi_{\text {off set,opt }}$ to its initial holographic surface of the design.

\subsection{FDA-approved single-element FUS transducer}

The first, most simple and conventional approach to deliver ultrasound to biological tissue is to use a single-element FUS transducer. The United States Food and Drug Administration (FDA) recently approved the use of a $0.25-\mathrm{MHz}$ single-element FUS transducer (a custom-designed device from Sonic Concepts: an outer diameter of $110 \mathrm{~mm}$, a radius of curvature of $110 \mathrm{~mm}$, an inner gap of $44 \mathrm{~mm}$ in diameter for the insertion of a passive cavitation detector (PCD) image transducer) for Alzheimer's treatment in adult humans. As the study in this chapter involves propagating FUS through a pediatric human skull, we choose the same FDA-approved transducer to assess its feasibility numerically for targeting a cancer tumor for a clinical trial. As the transducer presents a fixed focal length, we target the tumor through the skull's occipital region in order to position the focus in the center of the tumor. This transducer's position is fixed in all studied approaches using a holographic lens.

The pressure-field distribution using the single-element FUS transducer is shown in Fig. 4.2, where a clear focused beam in the center of the tumor is observed. The transmission through the skull does not affect the shape of the focus relevantly. No lateral lobes or secondary foci are observed, and the resulting focus has good symmetry and is centered with the transducer. These low aberrations are reasonable working at such a low frequency as $250 \mathrm{kHz}$. This case provides $V_{i n}=1.5 \mathrm{~cm}^{3}$, which 

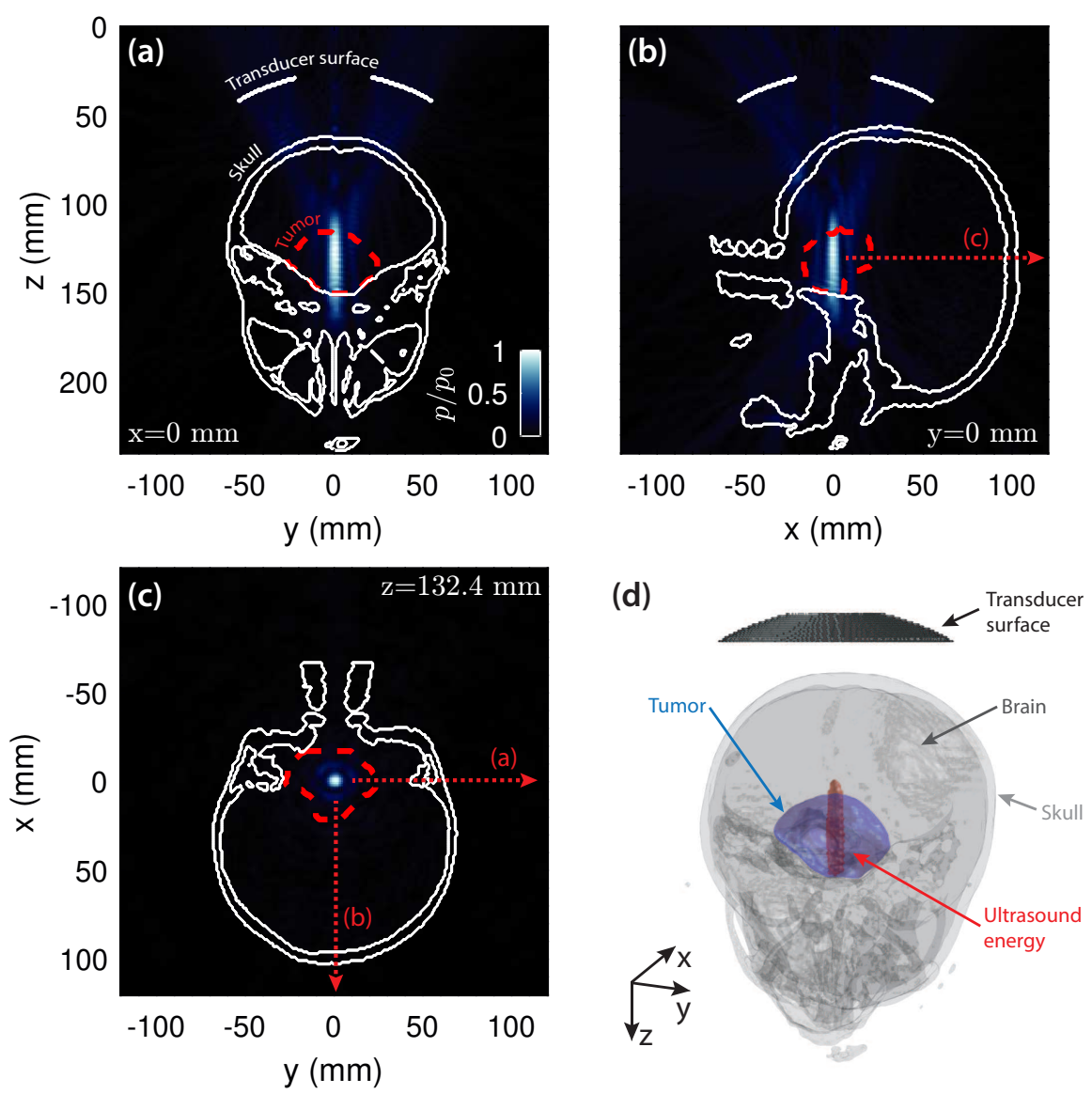

Figure 4.2: Pressure-field distribution using the conventional single-element FUS transducer. (a) Axial plane at $x=0 \mathrm{~mm}$, showing the transducer surface and skull boundaries (continuous white) and the tumor boundary (dashed red). (b) Sagittal plane at $y=0 \mathrm{~mm}$. (c) Coronal plane at $z=132.4 \mathrm{~mm}$. (d) 3D view of the transducer surface (black), skull (white), brain (gray) and focal volume (red) overlapping with the tumor (blue).

corresponds to $4.9 \%$ of the total tumor volume, and $V_{\text {out }}=0.2 \mathrm{~cm}^{3}$, representing $0.6 \%$ of the total tumor volume. This transducer has a linear 
focal gain of $G=7.4$, which means that the pressure at the surface of the transducer is linearly amplified by a factor of 7.4 in the focus. The focal volume shown in red in Fig. 4.2 (d) is obtained by thresholding the resulting pressure field between the maximum pressure value found in the tumor and the decay at its half. Therefore, if we adjust the input signal amplitude to get $0.5 \mathrm{MPa}$ at the maximum of the focus, the pressure at the boundary of the focal volume will correspond to 0.25 MPa. The BBB is expected to be opened with great accuracy just within this focal region. Quantitatively, for a gain of $G=7.4$, we need $0.07 \mathrm{MPa}$ at the surface of the transducer to ensure $0.5 \mathrm{MPa}$ at the maximum of the focus.

\subsection{Bifocal-hologram approach}

Starting from the easiest configuration, we assess the performance of a bifocal lens compared to the conventional case. This bifocal lens is firstly designed to create a simple shape of two foci covering the tumor (see Fig. 4.3). This lens design is calculated from the recorded back-propagated holographic wavefront at a flat surface in front of the transducer. To achieve a reduced $\mathrm{BBB}$ opening outside the tumor, the holographic information has been obtained applying the optimization function $f_{\text {opt }}$ (as described in Sec. 4.2.5) with $n=2$ to overlap at least twice the tumor volume than in the conventional case. This bifocal approach provides $V_{\text {in }}=3.5 \mathrm{~cm}^{3}$, representing $11.3 \%$ of the total tumor volume and 2.3 times greater than in the conventional case. In Fig. 4.3 (d), we can see the generation of a third focus in the center of both foci due to constructive interference of the converging wavefront coming from the lens, due to the symmetry of the acoustic image reconstruction, and because the two target points are very close to each other working at such a low frequency. The second quality parameter is $V_{\text {out }}=1.3 \mathrm{~cm}^{3}$, representing $4.3 \%$ of the total tumor volume and 7.1 times greater than in the conventional case. However, this value for $V_{\text {out }}$ represents $0.1 \%$ of the whole brain volume, which is acceptable from the medical point of view. Finally, $R A R=0.42$, therefore we need $0.16 \mathrm{MPa}$ at the surface of the transducer to ensure $0.5 \mathrm{MPa}$ at the maximum of the focus. We can see here that the pressure difference between focal and background regions has been reduced. Thus we have to be more accurate in the skull attenuation prediction to achieve the safe BBB 

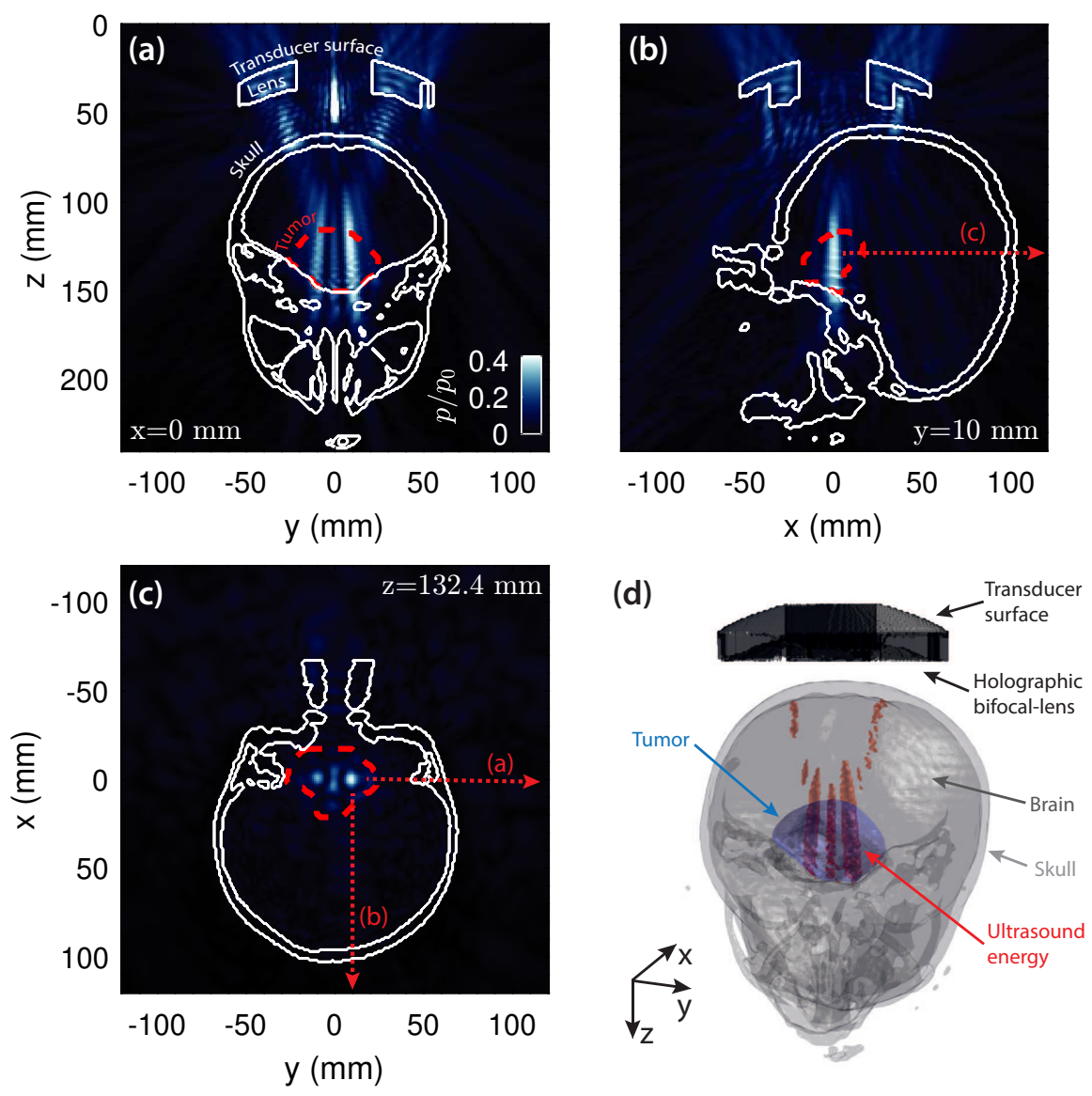

Figure 4.3: Pressure-field distribution, normalized to the maximum pressure $p_{0}$ at the focus in the conventional case, using the flat-design bifocal-hologram configuration. (a) Axial plane at $x=0 \mathrm{~mm}$, showing the transducer surface, lens and skull boundaries (continuous white) and the tumor boundary (dashed red). (b) Sagittal plane at $y=10 \mathrm{~mm}$. (c) Coronal plane at $z=132.4 \mathrm{~mm}$. (d) 3D view of the transducer surface (black), skull (white), brain (gray) and focal volume (red) overlapping with the tumor (blue). 
opening pressure range of $0.25-0.5 \mathrm{MPa}$ at the focal region. As expected, $R A R$ decreases because we are splitting the conventional single focus into two, in addition to the acoustic absorption of the lens material. Also, we observe how $V_{\text {in }}$ increases the same factor (2.3 times) as the inverse of $R A R$. In other words, the amplitude at the focus is reduced by the same factor that $V_{\text {in }}$ increases.

From the analysis of these quality parameters, we can say that the flat-design bifocal-hologram strategy allows an estimated 2.3 times (increase of $V_{i n}$ ) faster clinical procedure while being 2.3 times (decrease of $R A R)$ more accurate to ensure the delivery of $0.25-0.5 \mathrm{MPa}$ of pressure within the focal region.

\subsection{Volumetric slice-hologram approach}

Stepping towards a more adequate but complex holographic reconstruction, we assess the performance of a slice-shape lens compared to the conventional case. This lens is designed from a flat holographic surface and creates a complex broad focus along a slice of the tumor (see Fig. 4.4), although the reduced angular spectrum working at $250 \mathrm{kHz}$ does not allow to completely reconstructing the slice, resulting in a void in its center at $y=0 \mathrm{~mm}$ [see Fig. 4.4 (a,c)]. As in the previous bifocal approach, to achieve reduced $\mathrm{BBB}$ opening outside the tumor, the holographic information was obtained applying the optimization function $f_{\text {opt }}$ with $n=2.5$ (see Sec. 4.2.5). This approach provides a $V_{\text {in }}=4.0 \mathrm{~cm}^{3}$, representing $12.9 \%$ of the total tumor volume and 2.6 times greater than in the conventional case. The second quality parameter is $V_{\text {out }}=1.3 \mathrm{~cm}^{3}$, the same as in the bifocal approach. We can clearly see how $V_{i n}$ has increased while keeping the same $V_{\text {out }}$, so this slice-shape lens approach performs better. Finally, $R A R=0.40$, slightly smaller than the bifocal approach, therefore we need $0.18 \mathrm{MPa}$ at the surface of the transducer to ensure $0.5 \mathrm{MPa}$ at the maximum of the focus, and we can see here that the pressure difference between focal and background regions has been reduced a bit more. As expected, $R A R$ keeps decreasing because we are splitting the conventional single focus into a broad slice of the tumor, in addition to the acoustic absorption of the lens material. Moreover, we observe how $V_{\text {in }}$ increases more (2.6 times) than the inverse of $R A R(2.5)$, which means that the 

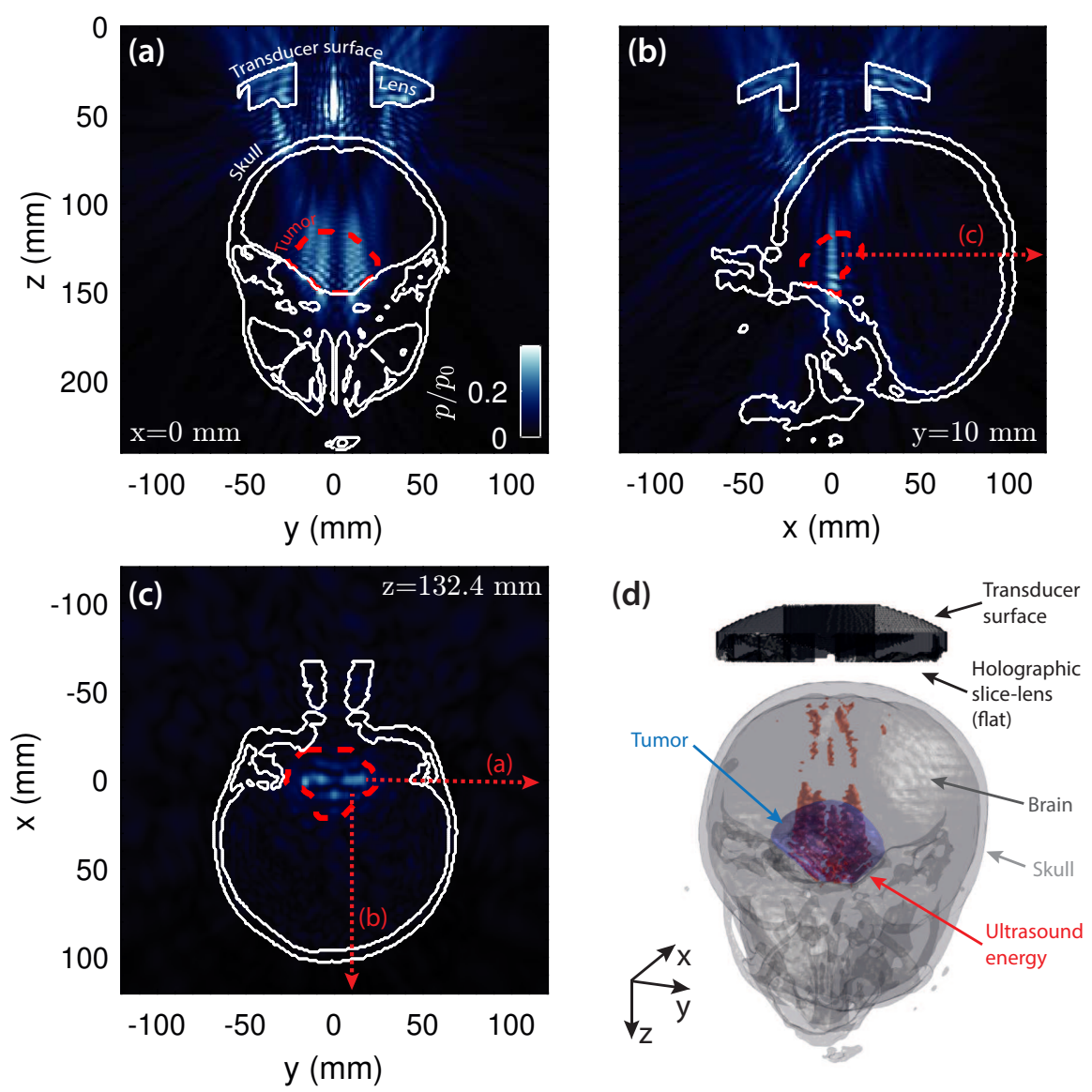

Figure 4.4: Pressure-field distribution, normalized to the maximum pressure $p_{0}$ at the focus in the conventional case, using the flat-design slice-hologram configuration. (a) Axial plane at $x=0 \mathrm{~mm}$, showing the transducer surface, lens and skull boundaries (continuous white) and the tumor boundary (dashed red). (b) Sagittal plane at $y=10 \mathrm{~mm}$. (c) Coronal plane at $z=132.4 \mathrm{~mm}$. (d) 3D view of the transducer surface (black), skull (white), brain (gray) and focal volume (red) overlapping with the tumor (blue). 
amplitude is higher than expected, as it does not decrease according to $R A R$. The pressure distributes efficiently and optimally along the slice of the tumor. This approach shows a better performance than the bifocal approach.

From the analysis of these quality parameters, we can say that the flat-design slice-hologram strategy allows 2.6 times (increase of $V_{i n}$ ) faster clinical procedure while being 2.5 times (decrease of $R A R$ ) more accurate to ensure the delivery of $0.25-0.5 \mathrm{MPa}$ of pressure within the focal region.

\subsubsection{Alternative slice-hologram approach}

Starting from the flat-design slice hologram, a new approach has been developed by recording the back-propagated holographic wavefront at a curved and parallel surface to the surface of the transducer, unlike done for the flat-design of the previous bifocal- and slice-hologram configurations. Then, we assess the performance of a curved-design slice-hologram compared to the conventional single-element FUS transducer approach. As well as the previous flat-design slice-hologram, this new design provides a complex, broad focus along the tumor (see Fig. 4.5), and this time, the reconstructed acoustic image shows a better homogeneity and the void in its center at $y=0 \mathrm{~mm}$ has almost disappeared [see Fig. 4.5 (a,c)]. Again, to achieve a reduced BBB opening outside the tumor, the holographic information was obtained applying the optimization function $f_{\text {opt }}$ with $n=2.5$ (see Sec. 4.2.5). This approach provides a $V_{i n}=3.9 \mathrm{~cm}^{3}$, representing $12.4 \%$ of the total tumor volume and 2.5 times greater than in the conventional case. The second quality parameter is $V_{\text {out }}=1.9 \mathrm{~cm}^{3}$, representing $6.2 \%$ of the total tumor volume, $\approx 10$ times greater than in the conventional case and $\approx 1.5$ times greater than in any of the previous holographic approaches, showing thus a poorer performance by covering quite more volume outside the tumor. However, this value for $V_{\text {out }}$ is still small compared to the size of the whole brain $(0.17 \%$ of it). Finally, $R A R=0.42$, the same as in the bifocal case. It is slightly higher than in the flat-design slice-hologram approach. Therefore, there is more amplitude at the tumor because this new lens design allows a thinner layer of lens material between transducer and holographic surfaces. Thus the ultrasound absorption is lower. Again, the amplitude at the tumor does not decrease according to $R A R$, and it is higher than expected, but $V_{\text {out }}$ 

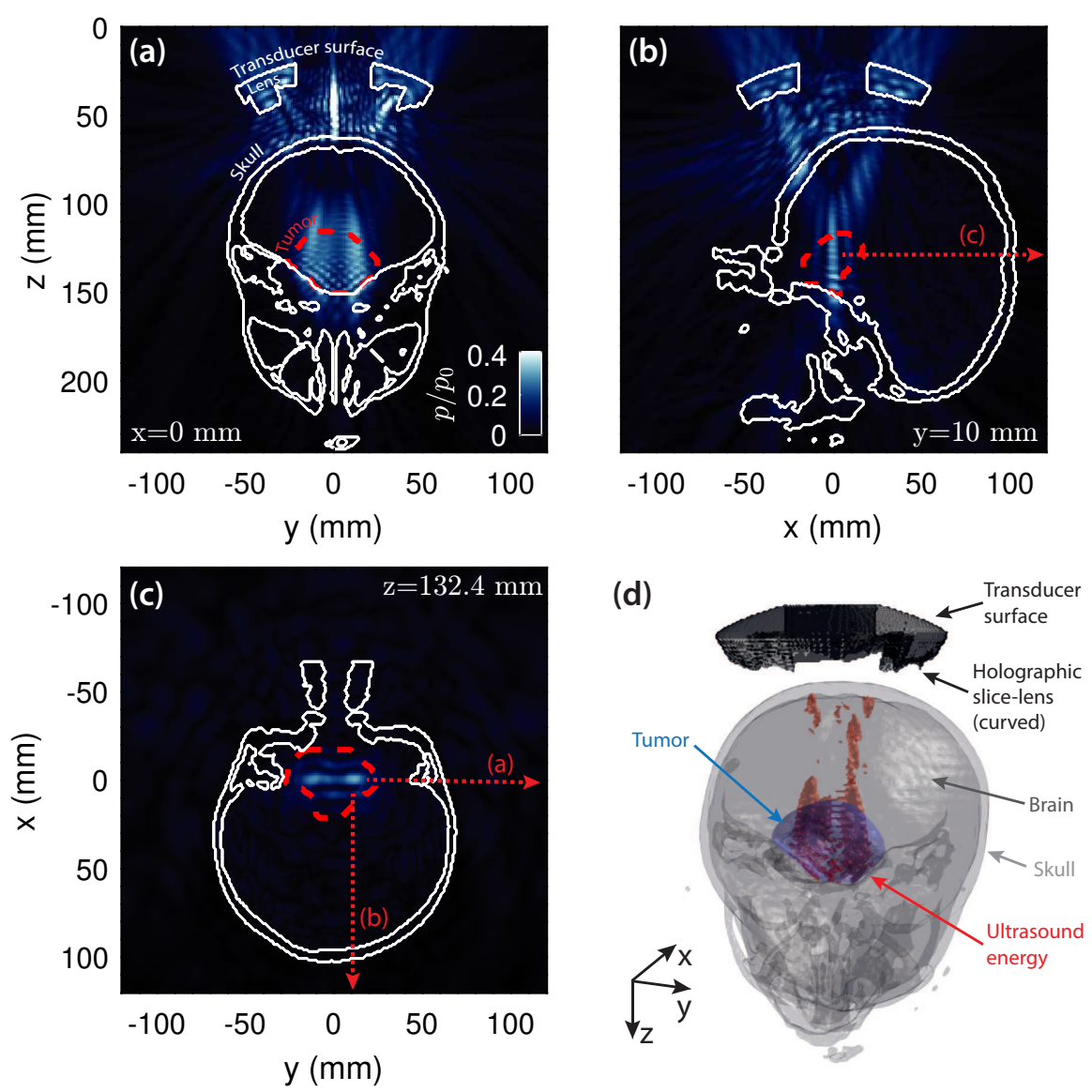

Figure 4.5: Pressure-field distribution, normalized to the maximum pressure $p_{0}$ at the focus in the conventional case, using the curved-design slice-hologram configuration. (a) Axial plane at $x=0 \mathrm{~mm}$, showing the transducer surface, lens and skull boundaries (continuous white) and the tumor boundary (dashed red). (b) Sagittal plane at $y=10 \mathrm{~mm}$. (c) Coronal plane at $z=132.4 \mathrm{~mm}$. (d) $3 \mathrm{D}$ view of the transducer surface (black), skull (white), brain (gray) and focal volume (red) overlapping with the tumor (blue). 
increases unnecessarily. There is no clear trend towards the better approach, because we are strongly limited by the transducer aperture at such a low frequency, but both flat- and curved-design slice-hologram approaches show a good performance.

From the analysis of these quality parameters, we can say that a curved-design slice-hologram allows 2.5 times (increase of $V_{i n}$ ) faster clinical procedure while being 2.4 times (decrease of $R A R$ ) more accurate to ensure the delivery of $0.25-0.5 \mathrm{MPa}$ of pressure within the focal region.

\subsubsection{Higher aperture feasibility approach}

As seen previously, the reduced aperture of the transducer, for such a low frequency, is a limiting factor for the design of the lens, as the results showed a good but not enough uniform distribution of the ultrasound beam along the tumor. The overall results showed a $V_{\text {in }}$ of $\approx 3$ times more using a holographic lens than the conventional transducer. However, $V_{\text {out }}$ was $\approx 7$ times higher. Moreover, the delivered pressure at the tumor was reduced by a factor of $\approx 3$, which implied that a very accurate prediction of the skull attenuation was required to ensure $0.25-0.5 \mathrm{MPa}$ within the focal region. Therefore, another approach was studied using a higher aperture transducer, keeping its central hole for the PCD and its radius of curvature. Although this new transducer is not FDA-approved, it is chosen to show the relevant improvements when the active aperture increases. As the aperture is higher, if the lens is designed from a flat holographic surface, as done in Sec. 4.5, the back part of the lens would be thicker, which would consequently increase the ultrasound absorption. For this approach, the lens has been designed from a curved holographic surface, as explained in Sec. 4.5.1, in order to avoid the undesired absorption increase inside the lens. The aperture of this transducer has been increased from $2 a=110 \mathrm{~mm}$ (FDA-approved transducer) to $2 a=132 \mathrm{~mm}$, a total of $22 \mathrm{~mm}$ in outer diameter. As well as the previous flat- and curved-design slice-holograms, this new lens is designed to create a complex broad focus along the tumor (as shown in Fig. 4.6). The result shows a uniform pressure distribution and a sharp focus [see Fig. $4.6(\mathrm{a}, \mathrm{c})]$. We observe how the ultrasound covers the entire slice of the tumor with great accurateness while reducing the off-target coverage. Again, to achieve a reduced $V_{\text {out }}$, the holographic information has been obtained applying the 

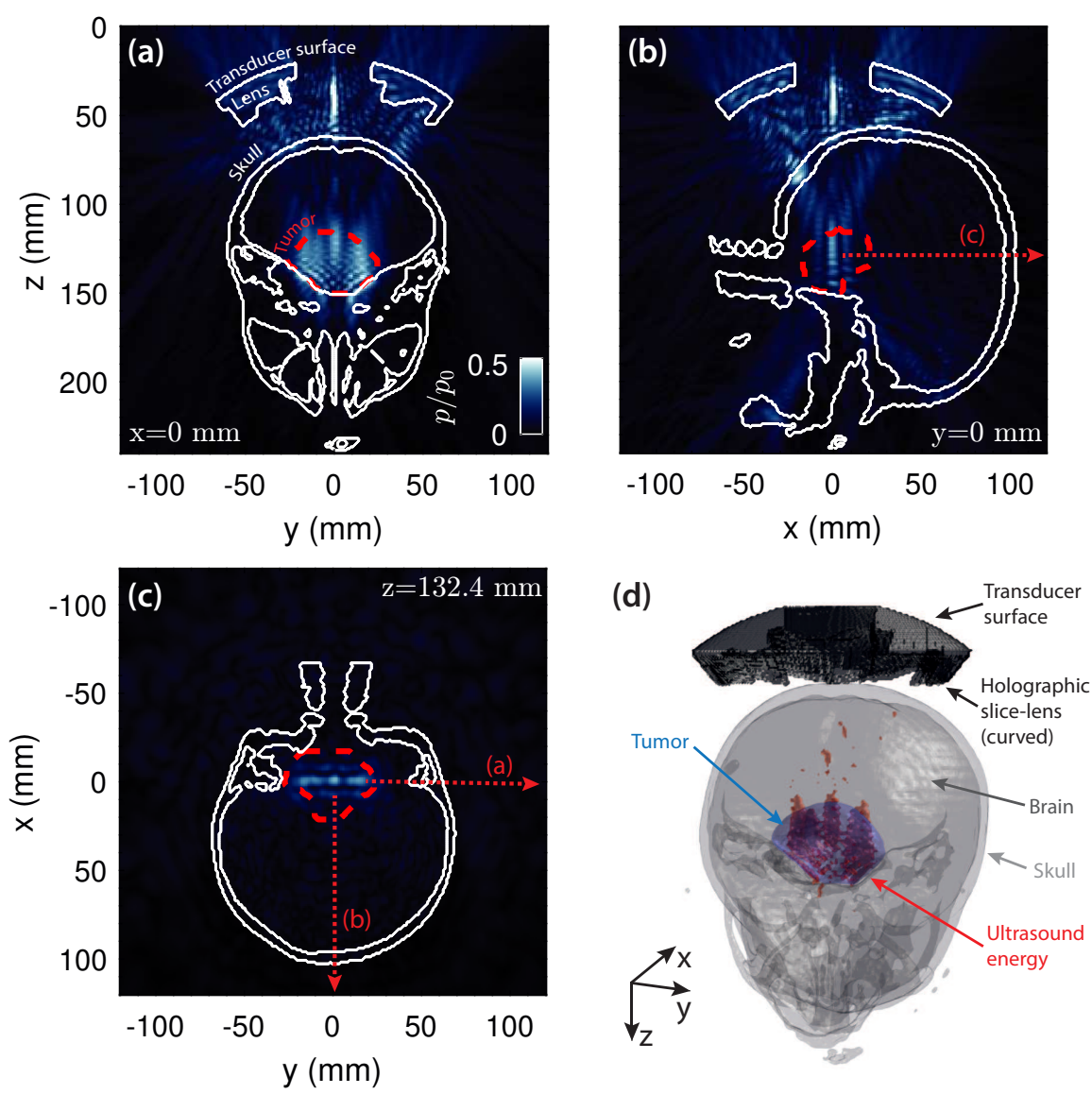

Figure 4.6: Pressure-field distribution, normalized to the maximum pressure $p_{0}$ at the focus in the conventional $(2 a=110 \mathrm{~mm})$ case, using a high-aperture curved-design slice-hologram configuration. (a) Axial plane at $x=0 \mathrm{~mm}$, showing the transducer surface, lens and skull boundaries (continuous white) and the tumor boundary (dashed red). (b) Sagittal plane at $y=0 \mathrm{~mm}$. (c) Coronal plane at $z=132.4 \mathrm{~mm}$. (d) $3 \mathrm{D}$ view of the transducer surface (black), skull (white), brain (gray) and focal volume (red) overlapping with the tumor (blue). 
optimization function $f_{\text {opt }}$ with $n=2.5$ (see Sec. 4.2.5). This approach provides a $V_{i n}=3.9 \mathrm{~cm}^{3}$, similar to previous cases. The second quality parameter is $V_{\text {out }}=0.8 \mathrm{~cm}^{3}, \approx 4$ times greater than in the conventional case and about a half lower than in any previous holographic approaches. This approach provides the same tumor coverage as previous cases, but considerably reduces the off-target coverage, showing a relevant improvement. Finally, $R A R=0.54$, again improving any previous cases by a factor of $\approx 1.3$. Thereby, this curved-design slice-hologram coupled to a high-aperture FUS transducer $(2 a=132 \mathrm{~mm})$ shows a great performance.

From the analysis of these quality parameters, we can say that the curved-design slice-hologram strategy with a higher aperture of $132 \mathrm{~mm}$ in outer diameter allows 2.6 times (increase of $V_{i n}$ ) faster clinical procedure while being 1.9 times (decrease of $R A R$ ) more accurate to ensure the delivery of $0.25-0.5 \mathrm{MPa}$ of pressure within the focal region.

\subsubsection{Higher and complete active aperture feasibility ap- proach}

Stepping even further, and assuming that the PCD could be non-confocally positioned and outside the spherical section of the FUS therapy transducer, the extra surface of the FUS transducer available in its center could be used to increase the active aperture. Therefore, the transducer for this approach has a complete semi-spherical active surface without the central hole. Again, to avoid the higher absorption of the back part of the lens, it has been designed from a curved holographic surface, as explained in Sec. 4.5.1. As well as in the previous case where we had a higher aperture while keeping the central hole, for this new approach we use a transducer with the same aperture of $2 a=132 \mathrm{~mm}$, but with no hole. The result shows a high-quality complex broad focus along the slice of the tumor (see Fig. 4.7). As we can observe, the focus shows an excellent in-target pressure homogeneity and a sharp difference between focal and background pressure regions in the three dimensions [see Fig. 4.7 (a,b,c)]. The ultrasound covers the entire slice of the tumor, while providing a reduced off-target delivery. Again, to achieve a reduced $V_{\text {out }}$, the holographic information was obtained applying the optimization function $f_{\text {opt }}$ with $n=2.5$ (see Sec. 4.2.5). This approach provides $V_{\text {in }}=4.9 \mathrm{~cm}^{3}$, representing $15.9 \%$ of the total tumor volume. This covered in-target 

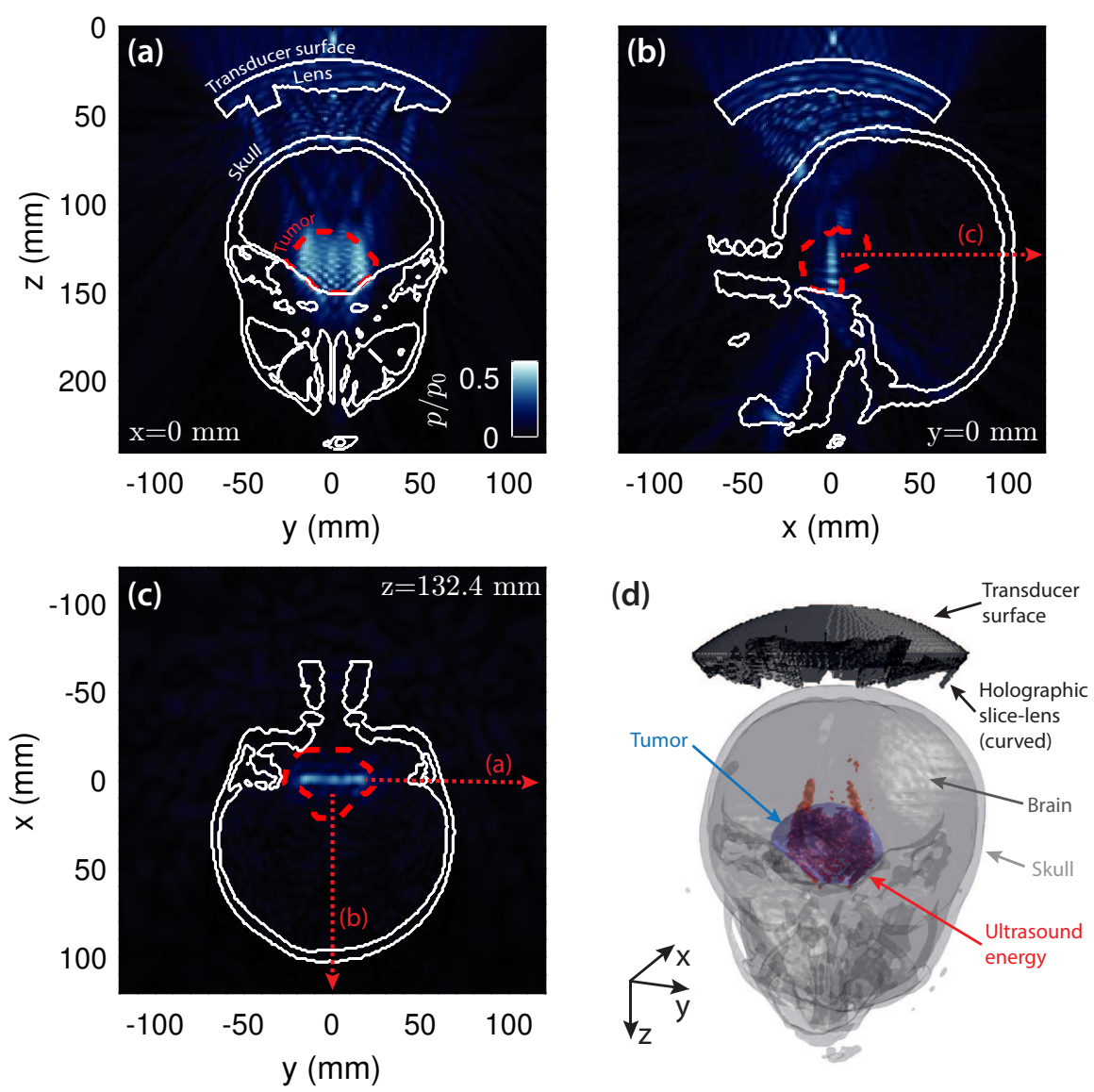

Figure 4.7: Pressure-field distribution, normalized to the maximum pressure $p_{0}$ at the focus in the conventional $(2 a=110 \mathrm{~mm})$ case, using the high- and complete-aperture curved-design slice-hologram configuration. (a) Axial plane at $x=0 \mathrm{~mm}$, showing the transducer surface, lens and skull boundaries (continuous white) and the tumor boundary (dashed red). (b) Sagittal plane at $y=0 \mathrm{~mm}$. (c) Coronal plane at $z=132.4 \mathrm{~mm}$. (d) $3 \mathrm{D}$ view of the transducer surface (black), skull (white), brain (gray) and focal volume (red) overlapping with the tumor (blue). 
volume is 3.3 times higher than in the reference case, i.e., the conventional FDA-approved single-element transducer with $2 a=110 \mathrm{~mm}$ and no lens, and $26 \%$ higher than in the previous approach with the central hole for the PCD. The second quality parameter is $V_{\text {out }}=0.7 \mathrm{~cm}^{3}$, $\approx 3.7$ times greater than in the reference case and $\approx 13 \%$ lower than in the previous case with the central hole. Therefore, the result provided by this approach is improved, as $V_{i n}$ is increased by a factor higher than the decreasing factor of $V_{\text {out }}$, i.e., increased tumor coverage while decreased off-tumor coverage. In addition, $R A R=0.64$, which implies a considerable improvement of any previous holographic approaches. Thereby, this curved-design slice-hologram coupled to a FUS transducer with a higher $(2 a=132 \mathrm{~mm})$ and complete (no hole) active aperture shows excellent performance.

From the analysis of these quality parameters, we can say that the curved-design slice-hologram strategy with a higher aperture of $132 \mathrm{~mm}$ in outer diameter, and without the central hole for the PCD, allows 3.3 times (increase of $V_{i n}$ ) faster clinical procedure, with no significant off-target ultrasound delivery, while being just 1.6 times (decrease of $R A R$ ) more accurate to ensure the delivery of $0.25-0.5 \mathrm{MPa}$ of pressure within the focal region.

\subsection{Discussion}

All studied approaches provide an increase of the tumor volume treated with ultrasound ( $\left.V_{i n} \uparrow \uparrow\right)$, a consequent increase in the treated off-target volume $\left(V_{\text {out }} \uparrow \uparrow\right)$, and a decrease in the maximum pressure obtained at the focus, as the focus is spread along the shape of the tumor $(R A R<<1)$.

The quality parameters characterize the feasibility of each studied approach to improve the BBB opening in a pediatric brain tumor compared to the FDA-approved approach [see Table(4.1)]. First, $V_{i n}$ is shown in $\mathrm{cm}^{3}$ and percentage relative to the total volume of the tumor $\left(V_{\text {tumor }}=\right.$ $31.1 \mathrm{~cm}^{3}$ ), in order to evaluate the overlapped volume between the focused ultrasound beam and the tumor. Second, $V_{\text {out }}$ is shown in $\mathrm{cm}^{3}$ to evaluate the amount of FUS delivered to brain tissues that do not belong to the tumor (off-target tissues). Finally, the relative focal amplitude reduction $R A R$ is shown to compare the provided focused beam gain between the 
Chapter 4. Hologram design for targeted chemotherapy

\begin{tabular}{l|ccc} 
& $V_{\text {in }}\left(\mathrm{cm}^{3}\right)$ & $V_{\text {out }}\left(\mathrm{cm}^{3}\right)$ & $R A R$ \\
\hline Conventional transducer & $1.5(4.9 \%)$ & 0.2 & 1.00 \\
\hline Flat-design bifocal & $3.5(11.3 \%)$ & 1.3 & 0.42 \\
\hline Flat-design slice & $4.0(12.9 \%)$ & 1.3 & 0.40 \\
\hline Curved-design slice & $3.9(12.4 \%)$ & 1.9 & 0.42 \\
\hline Curved-design slice $(2 a=132 \mathrm{~mm})$ & $3.9(12.4 \%)$ & 0.8 & 0.54 \\
\hline Curved-design slice $(2 a=132 \mathrm{~mm}$, no hole $)$ & $4.9(15.9 \%)$ & 0.7 & 0.64
\end{tabular}

Table 4.1: Quality parameters comparison between the reference case (FDA-approved) and the different approaches using a holographic acoustic lens. (Row 1) $V_{\text {in }}$ in $\mathrm{cm}^{3}$ and percentage to evaluate the covered volume inside the tumor. (Row 2) $V_{\text {out }}$ in $\mathrm{cm}^{3}$ to evaluate the covered volume outside the tumor. (Row 4) Relative focal amplitude reduction $R A R$ to evaluate the complexity to ensure the delivery of $0.25-0.5 \mathrm{MPa}$ of pressure within the focal region.

holographic approaches and the reference case. $R A R$ is used to assess the complexity to ensure accurate delivery of $0.25-0.5 \mathrm{MPa}$ within the focal region, as skull attenuation uncertainties are always present. Note that the aperture of the FDA-approved transducer in the conventional case is $2 a=110 \mathrm{~mm}$, compared to the two last studied holographic approaches where $2 a=132 \mathrm{~mm}$.

The reference FDA-approved case allows a covered volume of $1.5 \mathrm{~cm}^{3}$ in-target and $0.2 \mathrm{~cm}^{3}$ off-target. Thus, just $4.9 \%$ of the tumor, being required 21 sonications to cover the entire tumor, which would lead to a considerable effort (both in simulation predictions and experimental validation) to ensure correct positioning of the transducer for all the 21 different locations with respect to the patient's head, in addition to the several intravenously microbubble-injections required as well. The best approach to achieve a high $V_{\text {in }}$ while keeping a low $V_{\text {out }}$ compared to the reference case, is the curved-design slice-hologram coupled to a transducer with a higher aperture and no central hole. The new aperture would be $132 \mathrm{~mm}$ in outer diameter instead of $110 \mathrm{~mm}$. The PCD imaging transducer would be placed non-confocally and outside the complete aperture of the FUS transducer in order to work with more active radiating surface. This holographic approach allows coverage of $15.9 \%$ of the tumor, while the reference case would need 4 sonications to achieve the same 
tumor coverage. The performance of the reference case is thus very inefficient, and the clinical treatment would be very long. On the contrary, the great advantage of using the holographic lens with a higher aperture and without the central hole, is that just one single sonication and one single microbubble-injection would be enough to treat about 4 times more tumor volume.

\subsection{Conclusion}

The results presented in this chapter showed that any of the approaches using a holographic acoustic lens coupled to the $250 \mathrm{kHz}$ transducer for clinical trials could considerably improve the covered tumor volume. Consequently, the covered off-target volume also increased, although the lenses were thoughtfully designed to ensure a minimum off-target delivery, otherwise the BBB would be opened outside the tumor considerably. Note that the obtained off-target coverage for all the studied holographic approaches was much smaller than the in-target coverage. However, the focal gain was reduced about a half in all the cases, which is a relevant disadvantage as the gain of the conventional transducer with no lens was already reduced $(G=7.4)$. Therefore, it would be challenging to accurately deliver $0.25-0.5 \mathrm{MPa}$ within the tumor to achieve a controlled BBB opening due to existing uncertainties in the skull attenuation prediction and the reduced gain of the system. Nevertheless, the low ultrasound attenuation of a pediatric skull, softer than an adult one, and the low working frequency of $250 \mathrm{kHz}$, which is excellent for low-aberration purposes, would lead to overcome the skull attenuation uncertainties and provide a time- and cost-efficient clinical procedure.

Assuming a controlled FUS delivery within the tumor, the most suitable holographic approach to ensure a large tumor coverage with low off-target dispersion is the curved-design slice-hologram coupled to a transducer with $132 \mathrm{~mm}$ of aperture in outer diameter, $110 \mathrm{~mm}$ of radius of curvature, and no central hole for the PCD imaging transducer, achieving, therefore, coverage of $15.9 \%$ of the entire tumor by using a single sonication and a single microbubble-injection.

Acoustic holographic lenses have shown their capabilities to improve chemotherapy treatments, opening a new path to overcome the difficulties 
of treating pediatric brain tumors.

\section{References - Chapter 4}

[1] K. Beccaria, M. Canney, G. Bouchoux, C. Desseaux, J. Grill, A. B. Heimberger, and A. Carpentier, "Ultrasound-induced blood-brain barrier disruption for the treatment of gliomas and other primary cns tumors," Cancer Letters, 2020.

[2] D. N. Louis, A. Perry, G. Reifenberger, A. Von Deimling, D. FigarellaBranger, W. K. Cavenee, H. Ohgaki, O. D. Wiestler, P. Kleihues, and D. W. Ellison, "The 2016 world health organization classification of tumors of the central nervous system: a summary," Acta neuropathologica, vol. 131, no. 6, pp. 803-820, 2016.

[3] C. D. Arvanitis, G. B. Ferraro, and R. K. Jain, "The blood-brain barrier and blood-tumour barrier in brain tumours and metastases," Nature Reviews Cancer, vol. 20, no. 1, pp. 26-41, 2020.

[4] D. R. Groothuis, "The blood-brain and blood-tumor barriers: a review of strategies for increasing drug delivery," Neuro-oncology, vol. 2, no. 1, pp. 45$59,2000$.

[5] M. D. Prados, S. C. Schold Jr, H. A. Fine, K. Jaeckle, F. Hochberg, L. Mechtler, M. R. Fetell, S. Phuphanich, L. Feun, T. J. Janus, et al., "A randomized, double-blind, placebo-controlled, phase 2 study of rmp-7 in combination with carboplatin administered intravenously for the treatment of recurrent malignant glioma," Neuro-oncology, vol. 5, no. 2, pp. 96-103, 2003.

[6] A. Boiardi, A. Silvani, M. Eoli, E. Lamperti, A. Salmaggi, P. Gaviani, A. Fiumani, A. Botturi, C. Falcone, A. Solari, et al., "Treatment of recurrent glioblastoma: can local delivery of mitoxantrone improve survival?," Journal of neuro-oncology, vol. 88, no. 1, pp. 105-113, 2008.

[7] J. N. Bruce, R. L. Fine, P. Canoll, J. Yun, B. C. Kennedy, S. S. Rosenfeld, S. A. Sands, K. Surapaneni, R. Lai, C. L. Yanes, et al., "Regression of recurrent malignant gliomas with convection-enhanced delivery of topotecan," Neurosurgery, vol. 69, no. 6, pp. 1272-1280, 2011.

[8] J. D. Heiss, A. Jamshidi, S. Shah, S. Martin, P. L. Wolters, D. P. Argersinger, K. E. Warren, and R. R. Lonser, "Phase i trial of convection-enhanced delivery of il13-pseudomonas toxin in children with diffuse intrinsic pontine 
glioma," Journal of Neurosurgery: Pediatrics, vol. 23, no. 3, pp. 333-342, 2018.

[9] M. Tanter, M. Pernot, J.-F. Aubry, G. Montaldo, F. Marquet, and M. Fink, "Compensating for bone interfaces and respiratory motion in high-intensity focused ultrasound," International Journal of Hyperthermia, vol. 23, no. 2, pp. 141-151, 2007.

[10] S. Jiménez-Gambín, N. Jiménez, J. M. Benlloch, and F. Camarena, "Holograms to focus arbitrary ultrasonic fields through the skull," Physical Review Applied, vol. 12, no. 1, p. 014016, 2019.

[11] A. N. Pouliopoulos, S.-Y. Wu, M. T. Burgess, M. E. Karakatsani, H. A. Kamimura, and E. E. Konofagou, "A clinical system for non-invasive bloodbrain barrier opening using a neuronavigation-guided single-element focused ultrasound transducer," Ultrasound in medicine $\&$ biology, vol. 46, no. 1, pp. $73-89,2020$.

[12] R. S. Cobbold, Foundations of biomedical ultrasound. Oxford university press, 2006.

[13] P. A. Yushkevich, J. Piven, H. C. Hazlett, R. G. Smith, S. Ho, J. C. Gee, and G. Gerig, "User-guided 3d active contour segmentation of anatomical structures: significantly improved efficiency and reliability," Neuroimage, vol. 31, no. 3, pp. 1116-1128, 2006.

[14] M. E. M. Karakatsani, G. M. Samiotaki, M. E. Downs, V. P. Ferrera, and E. E. Konofagou, "Targeting effects on the volume of the focused ultrasoundinduced blood-brain barrier opening in nonhuman primatesin vivo," IEEE transactions on ultrasonics, ferroelectrics, and frequency control, vol. 64, no. 5, pp. 798-810, 2017.

[15] Cover image for this chapter, creator name: peterschreiber.media, stock.adobe.com.

\section{Publications}

The contents of this chapter were presented in the following publications: 


\section{Conference proceedings}

- S. Jiménez-Gambín, A. N. Pouliopoulos, Z. K. Englander, N. Jiménez, F. Camarena, E. E. Konofagou, S. Zacharoulis, and Cheng-Chia Wu, "Modeling Of Acoustic Holograms For Intensity-Modulated Ultrasound In Pediatric Brain Tumors". 20th International Symposium of ISTU, HICO, Gyeongju, Korea (virtual modality). Accepted poster presentation. June 6-9, 2021.

- S. Jiménez-Gambín, A. N. Pouliopoulos, Z. K. Englander, N. Jiménez, F. Camarena, E. E. Konofagou, S. Zacharoulis, and Cheng-Chia Wu, "Modeling of Intensity-Modulated Focused Ultrasound in Pediatric Brain Tumors Using Acoustic Holograms". IEEE International Ultrasonics Symposium (IUS), virtual symposium. Abstract submitted for acceptation. September 12-16, 2021. 



\subsection{Introduction}

Acoustic vortex beams are collimated wavefronts exhibiting phase dislocations, whose phase is proportional to $e^{i l \phi}$, with $\phi$ the azimuthal angle and $l$ the topological charge. The field exhibits a phase singularity at the beam axis and, therefore, it vanishes at this point due to destructive interference. Acoustic vortex beams carry angular orbital momentum $[1,2]$ that can be transferred to micro and macroscopic solid objects [3-8] or fluids [9] and exert mechanical torques to them. In addition, the singular scattering of vortex beams on small particles produces trapping acoustic radiation forces, resulting in practical applications for contactless particle tweezing and manipulation [10-13]. It is worth noting here that acoustic vortices can exert mechanical forces several orders of magnitude higher than those of their optical counterparts, with lower induced thermal effects $[14,15]$, and, furthermore, they can propagate deeply through opaque media such as biological tissues. Acoustic vortices have recently shown their potential to manipulate individual cells without inducing photothermal and/or photochemical damages [16], to trap and guide kidney stone fragments [17], or to selectively guide individual drug-delivery carriers based on microbubbles [18].

As the basis of these techniques is phase dislocations, the wavefront must be controlled with accuracy. Several methods to synthesize vortex beams have been reported, including active sources or phased arrays $[2,19-21]$, transducers with helical geometries [19, 22], Archimedean $[23,24]$ or Fresnel spiral diffraction gratings [25], systems exploiting the photoacoustic effect [26], active vibrating surfaces with spiral shape [27], or metamaterials exhibiting local resonances [28-32]. Acoustic holograms can generate vortex beams with great accuracy by encoding the full-wavefront information into the manufactured lens [33] [as it is also studied and demonstrated in Chapter 6].

However, when focusing into the brain, ultrasound beams experience strong refraction and attenuation due to the irregular and complex geometry of the skull, which introduces strong aberrations that distort the wavefront, decreasing the focusing performance of conventional single-element approaches. One strategy to solve this problem is using small invasive ultrasound implants located within the skull after partial craniotomy [34, 35] 
by directly bypassing the skull wall. This approach can be applied to enhance the delivery of chemotherapeutic agents after open surgery. However, in other circumstances, the need for craniotomy leads to severe complications. Non-invasive approaches include MRI-guided phased-array systems, which allow great accuracy in ultrasound focusing, beam steering, and the aberrations of the skull can be corrected by tuning the phase of each emitter [36, 37]. However, these systems typically consist of individual elements separated by a distance much greater than half the wavelength, presenting strong grating lobes when steering far from its geometrical focus. In addition, these systems are expensive and complex.

To solve this problem, structures showing local resonances, i.e., metamaterials, were theoretically proposed in 2D to compensate for the aberrations of a layer mimicking the skull [38]. However, its application in a realistic situation has not been demonstrated yet. Recently, 3D printed acoustic lenses have been proposed to mitigate the aberrations of the skull by focusing ultrasound beams at one single focal spot [39]. The full potential of 3D printed lenses for complex wavefront replication can be unleashed by using acoustic holograms [40]. Therefore, acoustic holograms can efficiently compensate for skull aberrations and, simultaneously, conform arbitrary acoustic images matching the geometry of the desired therapeutic target. In addition, as pixel density is much larger than state-of-the-art phased arrays for therapeutic ultrasound, acoustic holograms allow as well wide-angle beam steering without grating lobes [40].

In this chapter, we demonstrate that acoustic vortices can be focused through an ex-vivo human skull by using acoustic holographic lenses. Using personalized 3D-printed lenses, the phase aberrations produced by the skull can be compensated, and an acoustic beam containing phase dislocations can be focused through the skull.

\subsection{Hologram generation}

We design two holographic lenses, one propagating in homogeneous media (water) and another one propagating through the human skull. Following the methodology to design these lenses, as shown in previous chapters, we used the pseudo-spectral simulation method, including a $k$-space dispersion corrector and a time-reversal procedure [see Sec. 2.2 in Chapter 2]. 
For this chapter, the system is composed of a FUS piezoelectric transducer, custom made with a focal length of $F_{0}=140 \mathrm{~mm}$ and an aperture of $2 a_{0}=100 \mathrm{~mm}$, with a nominal frequency of $f_{0}=500 \mathrm{kHz}$, as sketched in Fig. 5.1 (a). The backward simulation step of the time-reversal approach is performed by using a set of 30 virtual sources located along a ring of radius $r_{s}=2.5 \mathrm{~mm}(0.83 \lambda)$ at the plane $z_{s}=F=97.4 \mathrm{~mm}(33 \lambda)$, centered at $x=y=0 \mathrm{~mm}$, as sketched in Fig. 5.1 (a). Each source was set to emit a sinusoidal signal with a complex amplitude proportional to $\exp (i l \theta)$. As we design a lens to be coupled to a spherically-focused transducer to obtain the holographic plane, we use the same method as explained in Sec. 3.2.3 in Chapter 3. So, the acoustic interference pattern produced by the virtual target sources, $H_{T}^{*}(x, y)$, was recorded at the holographic plane located at the exit plane of the transducer, given by $z_{0} F_{0}-\sqrt{F_{0}^{2}-a_{0}^{2}}$, resulting in the phase distribution shown in Fig. 5.1 (b). To compensate for the geometrical focusing of the bowl source (note $F \neq F_{0}$ ), we set an additional simulation for the focused transducer in the absence of skull, and we measured the field $H_{T}^{*}(x, y)$ at the exit plane $z_{0}$. The retrieved phase distribution is shown in Fig. 5.1 (c), presenting the characteristic Fresnel rings. The final hologram is designed by using

$$
H_{L}(x, y)=H_{T}^{*}(x, y) H_{F}^{*}(x, y),
$$

where $H_{T}^{*}(x, y)$ is the complex conjugate of the recorded wavefront at the holographic surface due to the effects of the virtual target sources and the skull, $(\cdot)^{*}$ denotes complex conjugation, and $x$ and $y$ are the transversal coordinates along the holographic plane. The resulting phase distribution is shown in Fig. 5.1 (d), where it can be visually identified the spiraling pattern due to the focused vortex and the wavy distortions produced by the aberrations of the skull.

We used a numerical grid with a spatial step of $\Delta x=\Delta y=\Delta z=$ $0.5 \mathrm{~mm}$ and a numerical temporal step of $\Delta t=33.2 \mathrm{~ns}$, leading to a Courant-Friedrichs-Lewy number of 0.10 in water and spatial sampling of six grid points per wavelength in water at $0.5 \mathrm{MHz}$. The lens surface is divided into squared pixels of $0.5 \times 0.5 \mathrm{~mm}^{2}$ of area. Therefore, the designed holographic acoustic lenses, with the central hole, have 31084 elements each, allowing accurate control of the holographic wavefront. Holographic acoustic lenses are first numerically designed, and afterward, manufac- 
Chapter 5. Transcranial holographic ultrasonic vortices
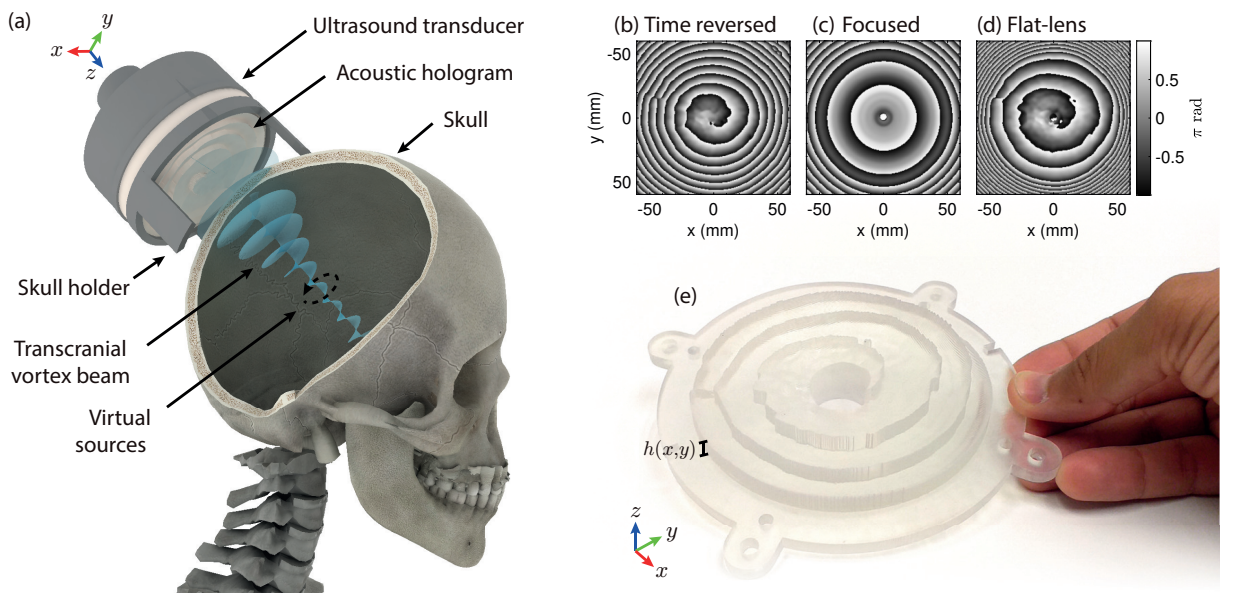

Figure 5.1: (a) Scheme of the setup. (b) Backward simulated field at the holographic plane, $H_{T}^{*}(x, y)$. (c) Field of the focused source, $H_{T}^{*}(x, y)$. (d) Phase-conjugated holographic field, $H_{L}(x, y)$. (e) 3D-printed acoustic hologram for the geometrically focused transducer.

tured using a 3D-printing stereo-lithographic technique (SLA) employing a Form 2 printer (Formlabs, USA), with a resolution of $50 \mu \mathrm{m}$ and $100 \mu \mathrm{m}$ in lateral and axial directions respectively, and using a photosensitive resin (Standard Clear, Formlabs, USA). The acoustical properties of the material were obtained experimentally using a pulse-echo technique in a test cylinder, resulting in a measured sound speed of $c_{L}=2580 \mathrm{~m} / \mathrm{s}$, density of $\rho_{L}=1171 \mathrm{~kg} / \mathrm{m}^{3}$ and absorption of $\alpha=1.38 \mathrm{~dB} / \mathrm{cm}$ at $0.5 \mathrm{MHz}$, matching the reported values of similar polymers [41, 42]. The final 3D-printed lens for the transcranial configuration is shown in Fig. 5.1(e).

For the skull acquisition, we scanned an ex-vivo human skull using $\mathrm{x}$-ray CT with an original resolution of $0.33 \times 0.33 \times 1.25 \mathrm{~mm}^{3}$, after the scalp was removed whole with a scalpel. This resolution was later interpolated using a cubic interpolation method to an isotropic grid of $0.5 \mathrm{~mm}$ $(\lambda / 6)$ for the simulations. Finally, these volumetric Hounsfield data were converted to bone density and sound speed values using empirical relations $[43,44]$. The sound speed ranges from $c_{0}$ to $c_{\max }=2958 \mathrm{~m} / \mathrm{s}$, with an average of $c_{\text {mean }}=2149 \mathrm{~m} / \mathrm{s}$, the density ranges from $\rho_{0}$ to $\rho_{\max }=2086 \mathrm{~kg} / \mathrm{m}^{3}$, with an average of $\rho_{\text {mean }}=1494 \mathrm{~kg} / \mathrm{m}^{3}$, match- 
ing those reported in literature $[45,46]$. The absorption of the skull is set to a value of $\alpha_{\text {skull }}=5.21 \mathrm{~dB} / \mathrm{cm}$ at $0.5 \mathrm{MHz}$ [47].

\subsection{Results}

After the two lenses are designed and manufactured, their performance is assessed in free media (first lens) and through the skull (second lens) by estimating the acoustic field using both a forward simulation and experimental measurements in a water tank.

\subsubsection{Vortices in homogeneous media}

The experimental setup for this homogeneous media validation is sketched in Fig. 5.4, but without placing the skull and using the lens designed for only-water propagation. The acoustic pressure field generated by the holographic acoustic lens for homogeneous media is shown in Fig. 5.2. The
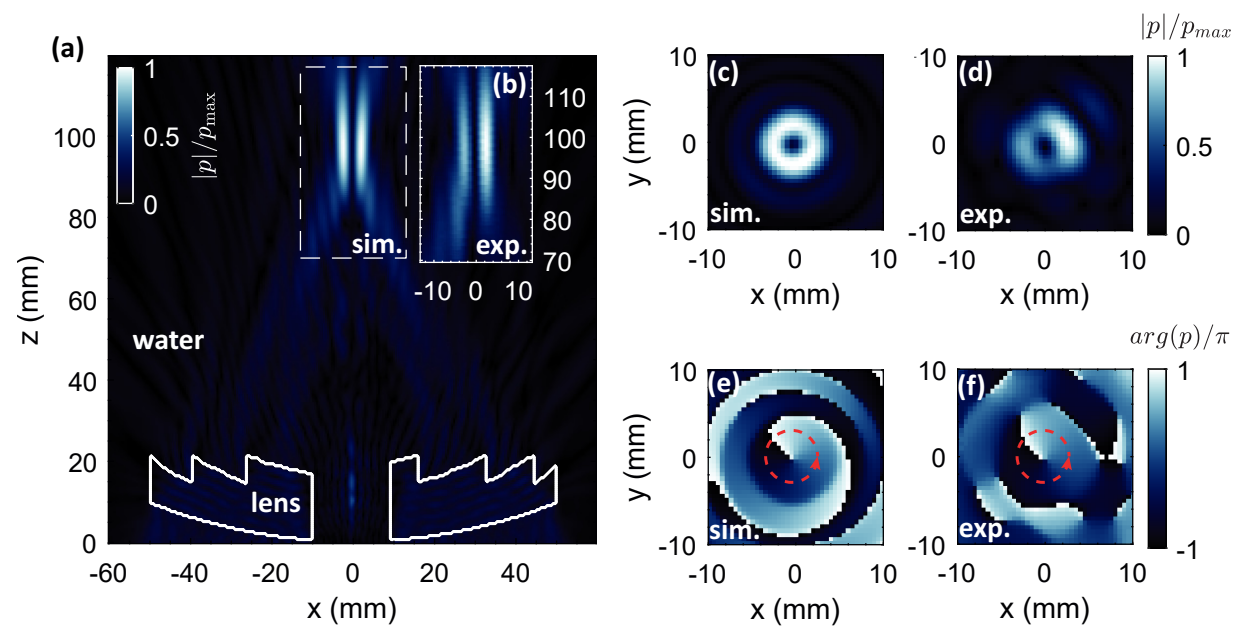

Figure 5.2: Axial and transversal planes showing the acoustic field of the vortex hologram $(l=1)$ designed for homogeneous media. (a) Simulated and (b) experimental normalized sagittal planes at $y=0 \mathrm{~mm}$. (c,d) Magnitude and $(e, f)$ phase of a transversal plane of the field obtained at $z=F$. 
magnitude of the sagittal field $\left(|p(x, 0, z)| / p_{\max }\right)$ is shown in Fig. 5.2 (a) for the simulation and experiment. We can identify that the designed hologram generates a void beam whose pressure peaks around the focal distance $(z=96.9 \mathrm{~mm}$ for the simulation and $z=97.0 \mathrm{~mm}$ for the experiment). Note that the geometrical focal of the source was $F_{0}=140 \mathrm{~mm}$. The transverse field profiles, measured at $z=F$, are given in Fig. 5.2 (c-f). The magnitude of the simulated and experimental field [Figs. $5.2(\mathrm{c}, \mathrm{d})]$ shows a ring-shaped distribution, and at its center, the field vanishes. The phase singularity at the focal spot causes a null, which is clearly visible in Figs. 5.2 (e,f) for the simulation and experiment, respectively. In addition, we can identify that the phase singularity is the result of a screw dislocation produced by the counter-clockwise rotation of the phase, marked by the red arrows.

In Fig. 5.3 (a) the magnitude of the field is shown in detail for a transverse cut. Experimental and simulated fields agree, and the beam presents a width of 1.5 times the wavelength at the nominal frequency. Finally, the phase along the azimuthal angle at a radial distance of $2.21 \mathrm{~mm}$, corresponding to the beam width, is shown in Fig. 5.3 (b). The generated transversal field shows a levorotatory phase distribution whose phase rotates as a function of the azimuthal angle at a rate given by the topological charge, $l=1$. Therefore, the phase rotates $2 \pi$ radians along a complete
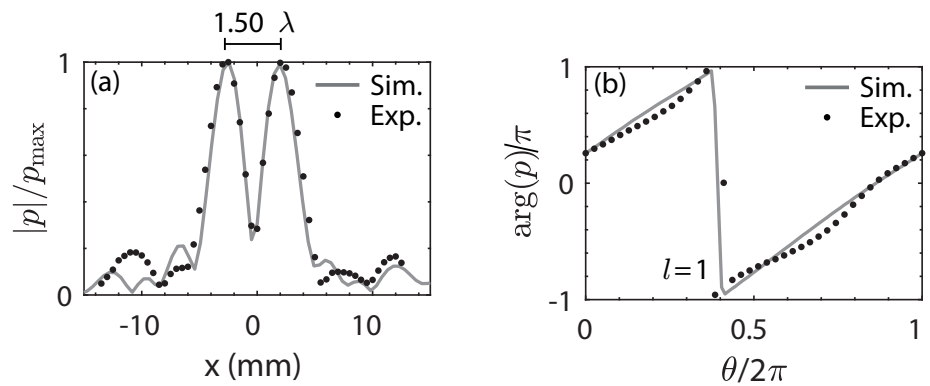

Figure 5.3: Transversal lines of the acoustic field of the vortex hologram $(l=1)$ designed for free media (water). (a) Normalized magnitude of the field at $z=F$ and $y=0 \mathrm{~mm}$. (b) Normalized phase of the field along the azimuthal angle. 
azimuthal turn, showing that the proposed lenses for moderately focused sources can conform acoustic vortices with great accuracy.

\subsubsection{Vortex synthesis in transcranial propagation}

For the experimental validation with the skull, the setup is sketched in Fig. 5.4.

The results of the lens designed for the transcranial propagation are summarized in Fig. 5.5. Figure 5.5 (a) shows the experimental setup, where the skull was immersed in a water tank, and the acoustic field was scanned in several planes using a hydrophone. A skull holder was 3D-printed to grant the correct positioning of the lens with respect to the surface of the skull. The irregular shape of the skull ensures that the holder can only be correctly located at the design position. The retrieved acoustic field is shown in Fig. 5.5 (b,c) for the simulation and experiment, respectively. The phase-only hologram can encode the time-reversed field with great accuracy. It compensates for the aberrations of the skull while,

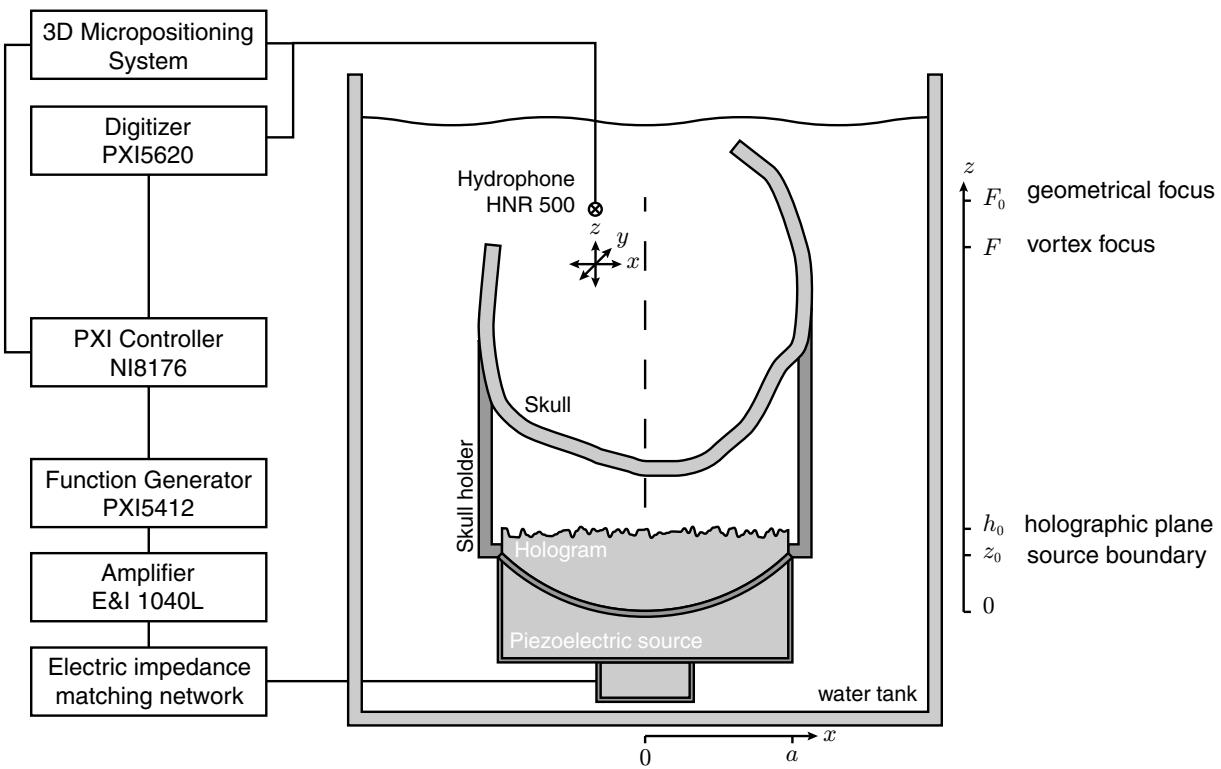

Figure 5.4: Scheme of the experimental setup. 
simultaneously, it modifies the wavefront to generate a focused vortex by introducing quasi-parabolic phase profile with rotational profile. In addition, simulation and experimental results are in good agreement. The pressure peaks at $z=96.9 \mathrm{~mm}$ for the simulation and $z=97.5 \mathrm{~mm}$ for the experiment. The transversal planes at $z=F$ are shown in Figs. $5.5(\mathrm{~d}-\mathrm{g})$. The transversal field magnitude [Figs. $5.5(\mathrm{~d}, \mathrm{e})]$ shows a ring-shaped distribution similar to the one obtained for homogeneous media. The magnitude of the field vanishes at the center for both experimental and simulated results. The corresponding phase distribution is shown in Fig. $5.5(\mathrm{f}, \mathrm{g})$.
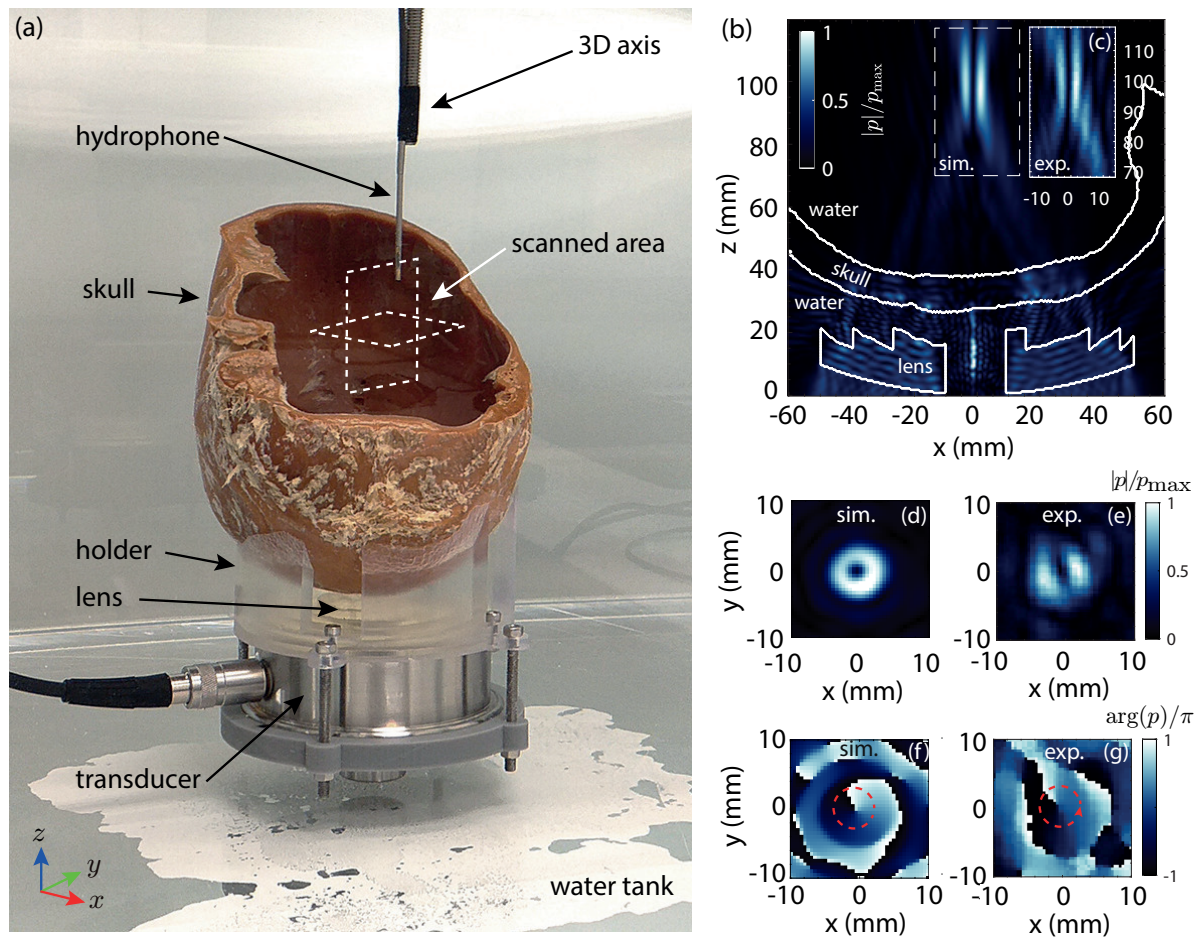

Figure 5.5: Field of the vortex hologram $(l=1)$ designed for transcranial propagation media. (a) Scheme of the experimental setup. (b) Simulated and (c) experimental normalized sagittal field plane at $y=0 \mathrm{~mm}$. (d,e) Magnitude and $(f, g)$ phase of a transversal plane of the field obtained at $z=F$. 
The counter-clockwise rotation is achieved at the focal plane, and a phase singularity is visible at the location of the null.

Note that in the absence of the skull, the simulated peak pressure is $7.7 p_{0}$, where $p_{0}$ is the pressure at the surface of the transducer. When introducing the skull, the simulated peak pressure decreased to $4.0 p_{0}$. In this way, the amplitude of the vortex decreases by a factor of $1.9(5.6 \mathrm{~dB})$ when introducing the skull.
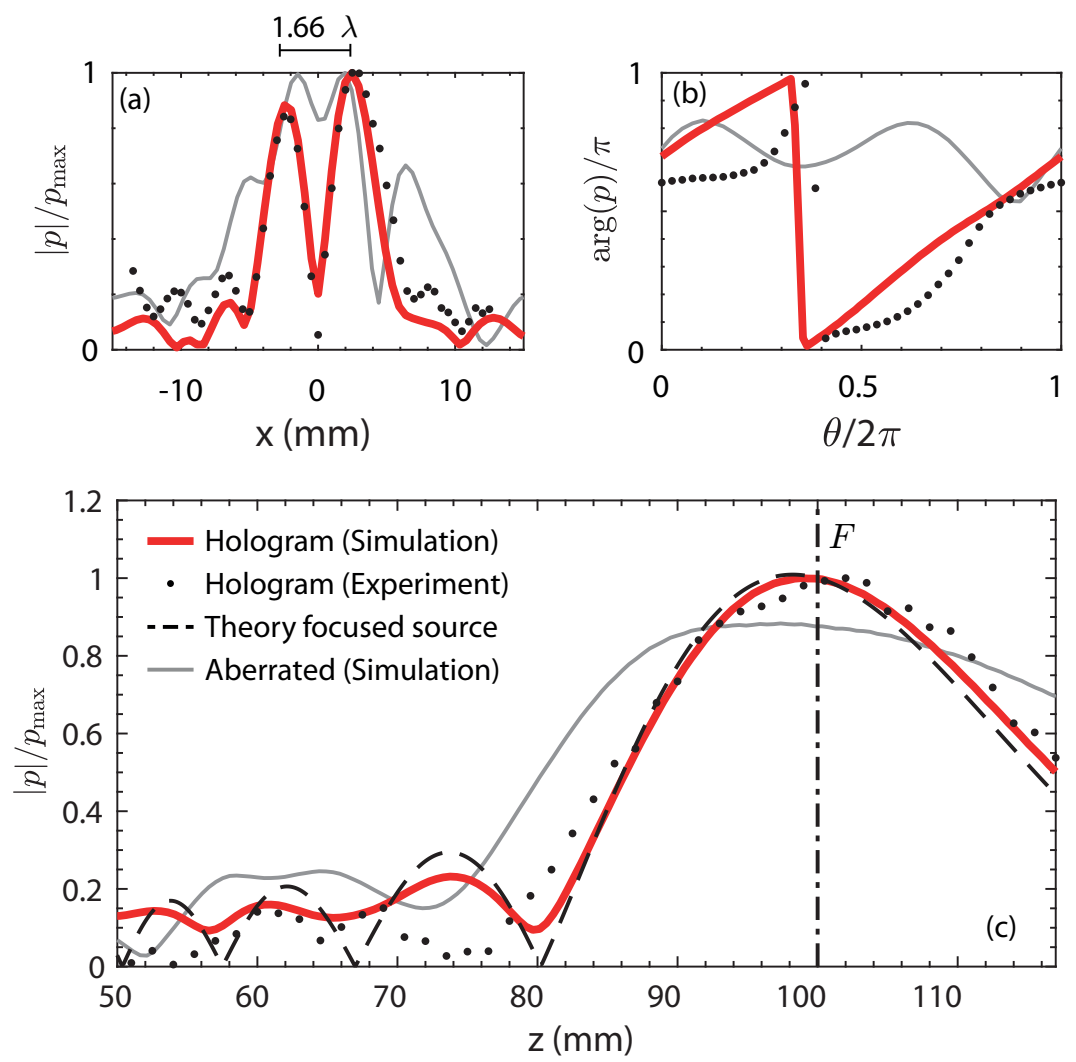

Figure 5.6: Detailed acoustic field analysis of the vortex hologram through the skull. (a) Normalized magnitude of the field at $z=F$ and $y=0 \mathrm{~mm}$. (b) Normalized phase of the field along the azimuthal angle. (c) Axial field line measured at $y=0 \mathrm{~mm}$ and $x=2.21 \mathrm{~mm}$. 
A detailed picture is shown in Fig. 5.6. The magnitude of the transversal field line cutting through the center of the null is shown in Fig. 5.6 (a). Experimental data agree with simulation results for this line. The experimental transversal width of the beam was $5 \mathrm{~mm}(1.66 \lambda)$, of the order of the value reached for the lens designed for homogeneous media. The experiment shows some discrepancies with the simulation (already visible when comparing Figs. $5.5(\mathrm{~d}, \mathrm{e})$ ). However, it is worth noting that when the lens designed for homogeneous media is used for the transcranial configuration [see the gray curve in Fig. 5.6 (a)], the aberrations distort the wavefront and the null is not observed. The phase of the field along the azimuthal angle is shown in Fig. 5.6 (b). The phase of the field generated for the transcranial hologram for both experiment and simulation rotates $2 \pi$ radians in a whole azimuthal turn. In contrast, the phase retrieved for the lens designed for homogeneous media does not show a screw dislocation because the skull aberrations distort the wavefront and avoid vortex generation. Finally, the longitudinal field line along the axial coordinate, measured at $y=0 \mathrm{~mm}$ and $x=2.5 \mathrm{~mm}$ (matching the peak pressure location), is shown in Fig. 5.6 (c). The experimental and simulated data for the transcranial hologram are also roughly in agreement with the theoretical field of a focused source in free media [48] given by

$$
|p(\tilde{z})|=2 p_{0}\left|\frac{\sin [G(1-\tilde{z}) /(2 \tilde{z})]}{1-\tilde{z}}\right|,
$$

where $\tilde{z}=z / F$ and $G=k a^{2} / 2 F$. The focusing performance of the lens is limited by the natural diffraction of waves, as occurs in any finite-aperture focused acoustic source. In contrast, using the lens designed for homogeneous media in the transcranial configuration, the aberrations produced by the skull distort the wavefront leading to a decrease in the focusing performance of the ultrasonic system.

\subsubsection{High-order topological charge transcranial vortex syn- thesis}

A more complex vortex-hologram is numerically designed to demonstrate the feasibility of this method to generate higher topological charge acoustic vortices. In particular, a topological charge of $l=2$ is chosen. For the backward simulation step of the time-reversal approach, a set of 44 virtual 
sources were located along a ring of radius $r_{s}=4.1 \mathrm{~mm}(1.39 \lambda)$ at the plane $z_{s}=F=97.4 \mathrm{~mm}(33 \lambda)$, centered at $x=y=0 \mathrm{~mm}$. Each source was set to emit a sinusoidal signal with a complex amplitude proportional to $\exp (i l \theta)$ with $l=2$.

A comparison of the simulated acoustic fields generated by the lenses for both homogeneous and aberrating media is shown in Fig. 5.7. The magnitude of the acoustic pressure sagittal fields $\left(|p(x, 0, z)| / p_{\max }\right)$ for water-only and transcranial is shown in Fig. 5.7 (a1,a2), respectively. We can identify that the designed hologram generates a void beam whose pressure peaks around the focal distance $(z=96.9 \mathrm{~mm}$ for water-only and $z=98.3 \mathrm{~mm}$ for transcranial). The transverse field planes, measured at $z=F$, are given in Figs. 5.7 (b1,b2,c1,c2). The magnitude of the water-only and transcranial fields [see Figs. 5.7 (b1,b2)] shows a ring-shaped distribution, and at its center, the field vanishes. The null is caused by the phase singularity at the focal spot, clearly visible in Figs. 5.7 (c1,c2) for the water-only and transcranial, respectively. In addition, we can identify that the phase singularity is the result of a screw dislocation produced by the counter-clock wise rotation of the phase, marked by the red arrows.

In Fig. 5.8 (a), the magnitude of the field is shown in detail for a
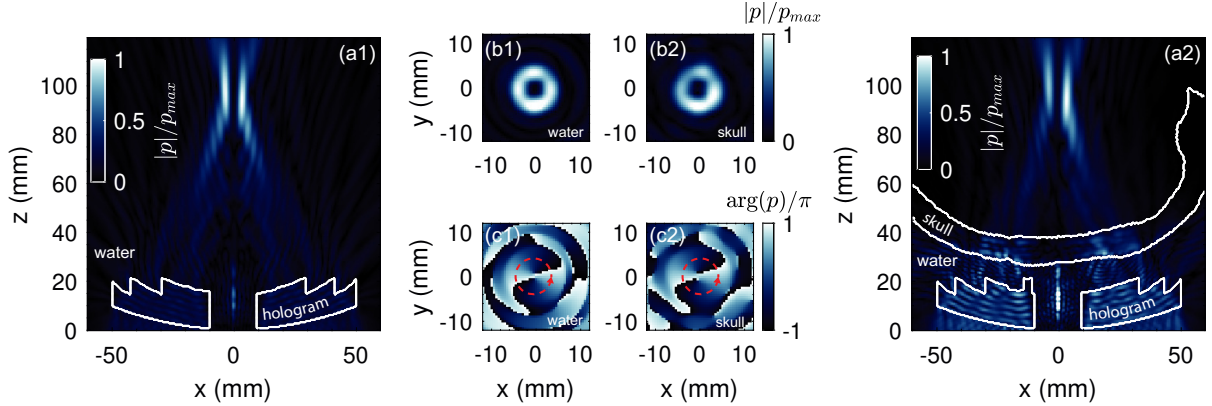

Figure 5.7: Simulated field of the vortex hologram $(l=2)$ designed for water-only and transcranial propagation media. (a1) Water-only and (a2) transcranial normalized sagittal field plane at $y=0 \mathrm{~mm}$. (b1,c1) Water-only and $(\mathrm{b} 2, \mathrm{c} 2)$ transcranial magnitude and phase, respectively, of a transversal plane of the field obtained at $z=F$. 
transversal line. Water-only and transcranial results agree, and the beam presents a width of $7.4 \mathrm{~mm}$ (2.5 times the wavelength at the nominal frequency). The phase along the azimuthal angle at a radial distance of $3.7 \mathrm{~mm}$, corresponding to half the beam width, is shown in detail in Fig. 5.8 (b). The generated transversal field shows a levorotatory phase distribution whose phase rotates as a function of the azimuthal angle at a rate given by the topological charge, $l=2$. Therefore, the phase rotates $2 \pi$ radians twice along a complete azimuthal turn, and water-only and transcranial results agree with high accuracy. Finally, the longitudinal field line along the axial coordinate, measured at $y=-3.7 \mathrm{~mm}$ and $x=$
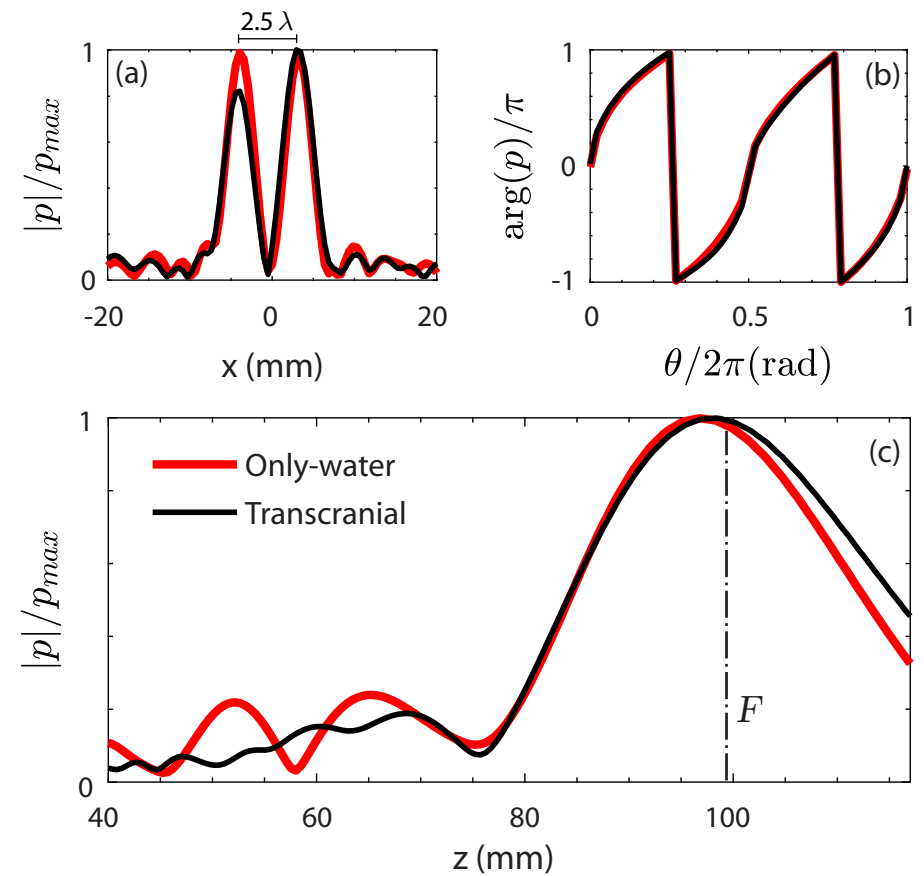

Figure 5.8: Detailed pressure-field lines for the water-only (red-continuous line) and transcranial (black-continuous line) holograms for the higher topological charge $l=2$. (a) Normalized magnitude of the field at $z=F$ and $y=0 \mathrm{~mm}$. (b) Normalized phase of the field along the azimuthal angle. (c) Axial field at $y=-3.7 \mathrm{~mm}$ and $x=0 \mathrm{~mm}$. 
$0 \mathrm{~mm}$ (matching the peak pressure location), is shown in Fig. 5.8 (c), where both water-only and transcranial results show a very similar focusing.

\subsection{Conclusions}

Human skull generates strong refraction and attenuation when ultrasonic waves propagate through it, resulting in aberrations that prevent the focusing of acoustical vortices at ultrasonic frequencies. As presented in this chapter, acoustic holograms can physically encode time-reversed fields containing phase dislocations with great accuracy using very simple and compact ultrasonic systems. On the one hand, holographic acoustic lenses allow compensating for the aberrations of the skull while, on the other hand, they simultaneously replicate the wavefront phase to generate focused vortex beams by introducing a quasi-parabolic phase with a rotational phase profile. This results in a personalized single-element system which that can focus a vortex through the human skull.

As the size of the acoustic vortices is limited by the natural diffraction of acoustic waves, it can be adjusted by changing the aperture and frequency of the source, i.e., by tuning the angular spectrum of the field encoded by the lens. In this situation, a personalized lens must be designed and manufactured for each ultrasound source and patient to obtain better targeting accuracy. In addition, we extended the lens design for vortices with higher topological charge, demonstrating its feasibility.

The holographic system described here can manipulate small particles in-vivo by mechanically steering the transducer+lens system to move the vortex location. Note that the correction of the skull aberrations can be maintained to some degree even if the source is moved with respect to the patient's head, as demonstrated recently for focused beams [49]. In this situation, an additional technology should be used to detect and locate the target, e.g., a clot. Portable ultrasonic methods or other imaging technologies such as CT or MRI could be applied, complementing the mechanical steering system and triggering the power-amplifier.

In addition, by focusing acoustic vortices, angular momentum can be transfer to tissues. Aberration-free holographic acoustic vortices open new venues to implement biomedical ultrasound applications beyond the transfer of linear momentum, allowing the transfer of angular momentum to the 
brain. The biological response of CNS tissues to the angular momentum produced by focused vortices remains unexplored. Other effects as neuromodulation or blood-brain barrier opening by trapping a cluster of bubbles inside the CNS using vortices might be studied in future works thanks to acoustic holography. In this way, the study described in this chapter will pave the road to design low-cost particle trapping applications, clot manipulation, torque exertion in the brain, and acoustic-radiation-force-based biomedical applications, opening the path to explore the biological response of the tissues to therapeutic ultrasonic vortex beams.

\section{References - Chapter 5}

[1] J. Nye and M. Berry, "Dislocations in wave trains," in Proceedings of the Royal Society of London A: Mathematical, Physical and Engineering Sciences, vol. 336, pp. 165-190, The Royal Society, 1974.

[2] J.-L. Thomas and R. Marchiano, "Pseudo angular momentum and topological charge conservation for nonlinear acoustical vortices," Physical review letters, vol. 91, no. 24, p. 244302, 2003.

[3] K. Volke-Sepúlveda, A. O. Santillán, and R. R. Boullosa, "Transfer of angular momentum to matter from acoustical vortices in free space," Phys. Rev. Lett., vol. 100, no. 2, p. 024302, 2008.

[4] K. Skeldon, C. Wilson, M. Edgar, and M. Padgett, "An acoustic spanner and its associated rotational doppler shift," New J. Phys., vol. 10, no. 1, p. $013018,2008$.

[5] L. Zhang and P. L. Marston, "Angular momentum flux of nonparaxial acoustic vortex beams and torques on axisymmetric objects," Phys. Rev. E, vol. 84, no. 6, p. 065601, 2011.

[6] A. Anhäuser, R. Wunenburger, and E. Brasselet, "Acoustic rotational manipulation using orbital angular momentum transfer," Phys. Rev. Lett., vol. 109, no. 3, p. 034301, 2012.

[7] C. E. Demore, Z. Yang, A. Volovick, S. Cochran, M. P. MacDonald, and G. C. Spalding, "Mechanical evidence of the orbital angular momentum to energy ratio of vortex beams," Phys. Rev. Lett., vol. 108, no. 19, p. 194301, 2012. 
[8] Z. Hong, J. Zhang, and B. W. Drinkwater, "Observation of orbital angular momentum transfer from bessel-shaped acoustic vortices to diphasic liquidmicroparticle mixtures," Phys. Rev. Lett., vol. 114, no. 21, p. 214301, 2015.

[9] A. Riaud, M. Baudoin, J.-L. Thomas, and O. B. Matar, "Cyclones and attractive streaming generated by acoustical vortices," Physical Review E, vol. 90, no. 1, p. 013008, 2014.

[10] J. Wu, "Acoustical tweezers," Jour. Acous. Soc. Am., vol. 89, no. 5, pp. 2140-2143, 1991.

[11] D. Baresch, J.-L. Thomas, and R. Marchiano, "Observation of a singlebeam gradient force acoustical trap for elastic particles: acoustical tweezers," Physical review letters, vol. 116, no. 2, p. 024301, 2016.

[12] A. Marzo, M. Caleap, and B. W. Drinkwater, "Acoustic virtual vortices with tunable orbital angular momentum for trapping of mie particles," Physical review letters, vol. 120, no. 4, p. 044301, 2018.

[13] Z. Gong and M. Baudoin, "Particle assembly with synchronized acoustic tweezers," Physical Review Applied, vol. 12, no. 2, p. 024045, 2019.

[14] A. Ashkin, "Acceleration and trapping of particles by radiation pressure," Physical review letters, vol. 24, no. 4, p. 156, 1970.

[15] D. G. Grier, "A revolution in optical manipulation," Nature, vol. 424, no. 6950, p. $810,2003$.

[16] M. Baudoin, J.-L. Thomas, R. Al Sahely, J.-C. Gerbedoen, Z. Gong, A. Sivery, O. B. Matar, N. Smagin, P. Favreau, and A. Vlandas, "Spatially selective manipulation of cells with single-beam acoustical tweezers," Nature Communications, vol. 11, no. 1, pp. 1-10, 2020.

[17] M. A. Ghanem, A. D. Maxwell, Y.-N. Wang, B. W. Cunitz, V. A. Khokhlova, O. A. Sapozhnikov, and M. R. Bailey, "Noninvasive acoustic manipulation of objects in a living body," Proceedings of the National Academy of Sciences, 2020 .

[18] D. Baresch and V. Garbin, "Acoustic trapping of microbubbles in complex environments and controlled payload release," Proceedings of the National Academy of Sciences, vol. 117, no. 27, pp. 15490-15496, 2020.

[19] B. T. Hefner and P. L. Marston, "An acoustical helicoidal wave transducer with applications for the alignment of ultrasonic and underwater systems," Jour. Acous. Soc. Am., vol. 106, no. 6, pp. 3313-3316, 1999. 
[20] R. Marchiano and J.-L. Thomas, "Synthesis and analysis of linear and nonlinear acoustical vortices," Phys. Rev. E, vol. 71, no. 6, p. 066616, 2005.

[21] J. F. Pazos-Ospina, J. L. Ealo, and E. E. Franco, "Characterization of phased array-steered acoustic vortex beams," The Journal of the Acoustical Society of America, vol. 142, no. 1, pp. 61-71, 2017.

[22] J. L. Ealo, J. C. Prieto, and F. Seco, "Airborne ultrasonic vortex generation using flexible ferroelectrets," IEEE transactions on ultrasonics, ferroelectrics, and frequency control, vol. 58, no. 8, pp. 1651-1657, 2011.

[23] N. Jiménez, R. Picó, V. Sánchez-Morcillo, V. Romero-García, L. M. GarcíaRaffi, and K. Staliunas, "Formation of high-order acoustic bessel beams by spiral diffraction gratings," Physical Review E, vol. 94, no. 5, p. 053004, 2016 .

[24] T. Wang, M. Ke, W. Li, Q. Yang, C. Qiu, and Z. Liu, "Particle manipulation with acoustic vortex beam induced by a brass plate with spiral shape structure," Applied Physics Letters, vol. 109, no. 12, p. 123506, 2016.

[25] N. Jiménez, V. Romero-García, L. M. García-Raffi, F. Camarena, and K. Staliunas, "Sharp acoustic vortex focusing by fresnel-spiral zone plates," Applied Physics Letters, vol. 112, no. 20, p. 204101, 2018.

[26] S. Gspan, A. Meyer, S. Bernet, and M. Ritsch-Marte, "Optoacoustic generation of a helicoidal ultrasonic beam," Jour. Acous. Soc. Am., vol. 115, no. 3, pp. 1142-1146, 2004.

[27] R. D. Muelas-Hurtado, J. L. Ealo, J. F. Pazos-Ospina, and K. VolkeSepúlveda, "Generation of multiple vortex beam by means of active diffraction gratings," Applied Physics Letters, vol. 112, no. 8, p. 084101, 2018.

[28] X. Jiang, Y. Li, B. Liang, J.-c. Cheng, and L. Zhang, "Convert acoustic resonances to orbital angular momentum," Phys. Rev. Lett., vol. 117, no. 3, p. 034301, 2016.

[29] L. Ye, C. Qiu, J. Lu, K. Tang, H. Jia, M. Ke, S. Peng, and Z. Liu, "Making sound vortices by metasurfaces," AIP Advances, vol. 6, no. 8, p. 085007, 2016 .

[30] A. Marzo, A. Ghobrial, L. Cox, M. Caleap, A. Croxford, and B. Drinkwater, "Realization of compact tractor beams using acoustic delay-lines," Appl. Phys. Lett., vol. 110, no. 1, p. 014102, 2017. 
[31] C. J. Naify, C. A. Rohde, T. P. Martin, M. Nicholas, M. D. Guild, and G. J. Orris, "Generation of topologically diverse acoustic vortex beams using a compact metamaterial aperture," Applied Physics Letters, vol. 108, no. 22, p. $223503,2016$.

[32] H. Esfahlani, H. Lissek, and J. R. Mosig, "Generation of acoustic helical wavefronts using metasurfaces," Physical Review B, vol. 95, no. 2, p. 024312 , 2017.

[33] S. Jiménez-Gambín, N. Jiménez, J. M. Benlloch, and F. Camarena, "Generating bessel beams with broad depth-of-field by using phase-only acoustic holograms," Scientific Reports, vol. 9, no. 1, pp. 1-13, 2019.

[34] C. Horodyckid, M. Canney, A. Vignot, R. Boisgard, A. Drier, G. Huberfeld, C. François, A. Prigent, M. D. Santin, C. Adam, et al., "Safe long-term repeated disruption of the blood-brain barrier using an implantable ultrasound device: a multiparametric study in a primate model," Journal of neurosurgery, vol. 126, no. 4, pp. 1351-1361, 2017.

[35] A. Idbaih, M. Canney, L. Belin, C. Desseaux, A. Vignot, G. Bouchoux, N. Asquier, B. Law-Ye, D. Leclercq, A. Bissery, et al., "Safety and feasibility of repeated and transient blood-brain barrier disruption by pulsed ultrasound in patients with recurrent glioblastoma," Clinical Cancer Research, vol. 25, no. 13, pp. 3793-3801, 2019.

[36] N. Lipsman, Y. Meng, A. J. Bethune, Y. Huang, B. Lam, M. Masellis, N. Herrmann, C. Heyn, I. Aubert, A. Boutet, et al., "Blood-brain barrier opening in alzheimer's disease using mr-guided focused ultrasound," Nature communications, vol. 9, no. 1, pp. 1-8, 2018.

[37] A. Abrahao, Y. Meng, M. Llinas, Y. Huang, C. Hamani, T. Mainprize, I. Aubert, C. Heyn, S. E. Black, K. Hynynen, et al., "First-in-human trial of blood-brain barrier opening in amyotrophic lateral sclerosis using mr-guided focused ultrasound," Nature communications, vol. 10, no. 1, pp. 1-9, 2019.

[38] C. Shen, J. Xu, N. X. Fang, and Y. Jing, "Anisotropic complementary acoustic metamaterial for canceling out aberrating layers," Physical Review X, vol. 4, no. 4, p. 041033, 2014.

[39] G. Maimbourg, A. Houdouin, T. Deffieux, M. Tanter, and J.-F. Aubry, "3dprinted adaptive acoustic lens as a disruptive technology for transcranial ultrasound therapy using single-element transducers," Physics in Medicine E Biology, vol. 63, p. 025026, jan 2018. 
[40] S. Jiménez-Gambín, N. Jiménez, J. M. Benlloch, and F. Camarena, "Holograms to focus arbitrary ultrasonic fields through the skull," Physical Review Applied, vol. 12, no. 1, p. 014016, 2019.

[41] M. Ferri, J. M. Bravo, J. Redondo, S. Jiménez-Gambín, N. Jiménez, F. Camarena, and J. V. Sánchez-Pérez, "On the evaluation of the suitability of the materials used to $3 \mathrm{~d}$ print holographic acoustic lenses to correct transcranial focused ultrasound aberrations," Polymers, vol. 11, no. 9, p. 1521, 2019.

[42] K. Melde, A. G. Mark, T. Qiu, and P. Fischer, "Holograms for acoustics," Nature, vol. 537, no. 7621, pp. 518-522, 2016.

[43] U. Schneider, E. Pedroni, and A. Lomax, "The calibration of CT hounsfield units for radiotherapy treatment planning," Physics in Medicine and Biology, vol. 41, pp. 111-124, jan 1996.

[44] T. D. Mast, "Empirical relationships between acoustic parameters in human soft tissues," Acoustics Research Letters Online, vol. 1, no. 2, pp. 37-42, 2000 .

[45] J. Hill, "Physical activity and obesity," The Lancet, vol. 363, no. 9404, p. 182, 2004 .

[46] A. Bouakaz and J.-M. Escoffre, "Therapeutic ultrasound," 2016.

[47] R. S. Cobbold, Foundations of biomedical ultrasound. Oxford university press, 2006.

[48] H. O'Neil, "Theory of focusing radiators," The Journal of the Acoustical Society of America, vol. 21, no. 5, pp. 516-526, 1949.

[49] G. Maimbourg, A. Houdouin, T. Deffieux, M. Tanter, and J.-F. Aubry, "Steering capabilities of an acoustic lens for transcranial therapy: numerical and experimental studies," IEEE Transactions on Biomedical Engineering, vol. 67, no. 1, pp. 27-37, 2019.

[50] Cover image for this chapter, creator name: Igor Stepovik, stock.adobe.com.

\section{Publications}

The contents of this chapter, and closely related ones, were presented in the following publications: 


\section{Journal papers}

- S. Jiménez-Gambín, N. Jiménez, and F. Camarena, "Transcranial focusing of ultrasonic vortices by acoustic holograms". Physical Review Applied, vol. 14, no. 5, p. 054070, 2020.

\section{Conference proceedings}

- N. Jiménez, S. Jiménez-Gambín, D. Andrés, and F. Camarena, "Conformation of ultrasonic vortex beams through the skull using acoustic holograms for particle trapping". "7th International Symposium on Focused Ultrasound, virtual symposium. November 8-13, 2020.

- N. Jiménez, S. Jiménez-Gambín, A. Cristian, and F. Camarena, "Vortices for biomedical ultrasound applications". 51 Congreso Español de Acústica; TECNIACUSTICA 2020; XI Congreso Ibérico de Acústica, virtual congress. June 3-5, 2020.

- G. Sánchez-Rodríguez, N. Jiménez, S. Jiménez-Gambín, and F. Camarena, "Arrays of vortices for particle trapping and patterning using single-beam acoustic holograms". 51 Congreso Español de Acústica; TECNIACUSTICA 2020; XI Congreso Ibérico de Acústica, virtual congress. June 3-5, 2020. 




\section{Chapter 6}

\section{Broad depth-of-field Bessel beams using acoustic holograms}

While a Bessel function correctly describes the transverse field distribution of Bessel beams generated using traditional passive methods, the axial distribution is not constant. In this chapter, acoustic holograms show the generation of zero-th and high-order truncated acoustic Bessel beams with flat-intensity along their axis. In particular, the results showed an elongated focal length of about 40 wavelengths, while the transverse width of the beam remained smaller than 0.7 wavelengths. In addition, first-order Bessel beams with a phase dislocation along their axis and a vortex with a single topological charge were also reported. The proposed method may have potential applications in ultrasonic imaging, biomedical ultrasound and particle manipulation applications using passive lenses. 


\subsection{Introduction}

Bessel functions are exact and invariant solutions of the Helmholtz equation $[1,2]$, i.e., an ideal Bessel beam does not experience diffraction. These beams present remarkable properties as self-healing, beam-width close to the diffraction limit, and excellent depth-of-field. In addition, high-order Bessel beams present phase dislocations, i.e., they conform vortex beams and transport orbital angular momentum. First proposed by Durnin in 1987 [1], Bessel beams have been broadly studied in both optics [1-6] and acoustics [7-9]. In the particular case of acoustics, they have found practical applications in ultrasound imaging systems [10-14]. Their long depth-of-field and narrow beam-width allow an accurate scanning of the transmitted beam, while their self-healing properties grant remarkable robustness to tissue scattering and its diffraction-free properties offer an almost constant imaging resolution with depth. Moreover, in recent years vortex beams and, in particular, Bessel beams have attracted attention due to their unique properties for particle trapping, manipulation, or rotation applications [15-25] or acoustic radiation force applications in fluids [26]. Vortex beams have also been proposed for robust acoustic communications [27, 28].

An ideal Bessel beam is generated by a converging conical wavefront of infinite extent. When it converges to the axis of symmetry and interferes with itself, the tilted conical wavefront generates the characteristic Bessel beam pattern. Thus, Bessel beams in the far-field are characterized by a single-ring pattern arising from their narrow angular spectrum. However, to generate ideal Bessel beams, an infinite amount of energy is needed. In practice, truncated Bessel beams are generated using finite-aperture sources or lenses, as shown in Fig. 6.1 (a).

In acoustics, there exist many methods to generate zeroth and high-order Bessel beams. While, as mentioned, they cannot be ideally generated in practice, several methods have been proposed to generate truncated Bessel beams. A straightforward strategy is to use a circular slit: at the axis, a Bessel beam pattern is generated, in analogy with optics [2]. However, the small aperture strongly limits the transmitted energy. Bessel beams were efficiently generated using arrays of annular active piezoelectric sources $[10-12,22,29-32]$. In these cases, each of the annular active 
(a) Truncated Bessel beam

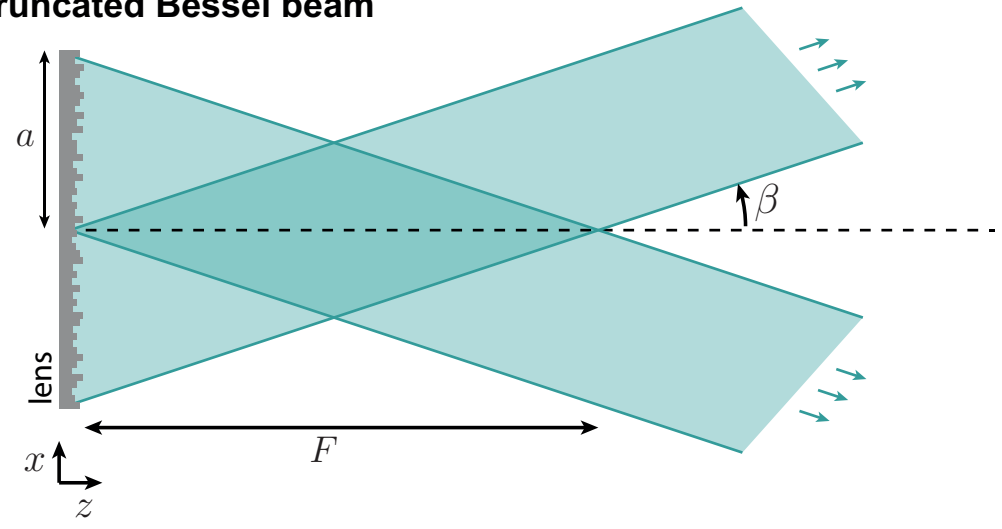

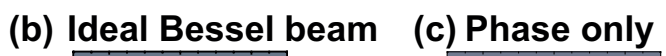
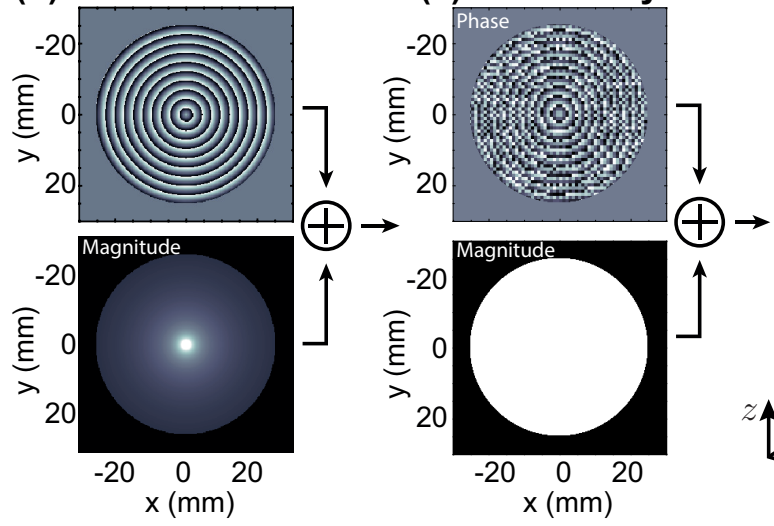

(d) Lens construction

Figure 6.1: (a) Scheme of a truncated Bessel beam for a monochromatic wave. Lens generation process: (b) Phase and magnitude of an ideal acoustical axicon, (c) resulting phase and magnitude after processing, (d) manufactured holographic phase-only lens and its geometrical definition.

elements is set according to a Bessel function in both, phase and amplitude. This process can be mimicked using simple discs by realising the similarity between the Bessel function and the radial modes of piezoelectric sources, though the radiation efficiency is low [33].

Beyond active arrays of transducers, Bessel beams can also be obtained by coupling a passive lens to a single active source. Examples are 
acoustical axicons [34, 35], in analogy with optical ones [36, 37]. Acoustic axicons are conical-shaped lenses: when waves are refracted along their tilted surface, a conical wavefront is generated. Variations of axicons are fraxicon lenses [38]. Fraxicons, in analogy to Fresnel lenses, are stepped phase plates able to produce a similar conical wavefront due to the refraction along its sawtooth-shaped profile. In this way, the thickness of the lens can be strongly reduced [39].

Instead of using refraction processes, Bessel beams can be obtained by using the diffraction of waves on axisymmetric gratings [40]. These lenses produce Bessel beams in a broad range of frequencies with frequency-dependent properties. Other approaches include the combination of a focused source with a layered structure [41]. The main drawback of these approaches is that the resulting beams could present aberrations due to multiple reflections inside the bulk of the lenses. Finally, metasurfaces have been proposed to generate zeroth-order Bessel beams using the reflection of sound waves in the air [42]. However, their performances are usually limited to a very narrow frequency band based on local resonances.

Acoustic beams can carry phase dislocations producing acoustic vortices [43]. In these conditions the beam carries pseudo-angular momentum [18], as occurs in the case of high-order Bessel beams. High-order Bessel beams can be analogously generated using Archimedes' spiral gratings where the order of the Bessel beam, i.e., the topological charge of the beam, is proportional to the number of arms of the spiral [44-48]. Equivalently, active elements with spiral geometries can also generate high-order Bessel beams $[49,50]$. The phase rotation needed to generate high-order Bessel beams can be generated using phase plates [51, 52]. Developable helicoidal/conical air-coupled active surfaces with phase dislocations have been also proposed using curved piezoceramics [53] or flexible ferroelectrets [54]. Finally, recently metasurfaces have been proposed to engineer the phase to generate acoustic vortices [55-58].

While a Bessel function accurately describes the field distribution in the transverse direction of the beam produced by these passive methods, all present a common drawback: the axial distribution of the field is not constant as required by the Bessel beam solution initially proposed by Durnin. In this way, the field-of-view is limited and these beams present a focal spot. In particular, using the cited passive methods to generate the 
beam, its intensity grows roughly linear with space. This is a geometric consequence of the axisymmetric converging wavefront: to maintain constant the field along the axis, the energy contained in all annular regions of the circular aperture should be constant.

In this work, we present a simple method to generate zeroth and high-order Bessel beams of flat-intensity along their axis using phase-only acoustic holograms. This kind of lens has recently been proposed to generate arbitrary acoustic fields and, simultaneously, correct the strong aberrations during transcranial ultrasound propagation both with phase-and-amplitude encoding [59] or only with phase encoding [60,61]. Other applications of holographic phase-only lenses include particle manipulation applications [62], multi-frequency focal generation [63], or photoacoustic generation of complex holographic fields [64]. Magnitude-and-phase lenses based on metamaterials have been proposed to control sound waves in the air [65]. However, their applicability in biomedical ultrasound is still minimal mainly due to the deep-subwavelength geometrical features of the resulting structures. In addition, most of the designs for metamaterials in air, where the solid phase can be considered perfectly rigid in most cases, are not directly applicable in biomedical ultrasound because it must be included the coupling between the solid metamaterial structure and either water or water-like tissues. Acoustic holograms present a robust and straightforward approach for these applications. Recently, phase and amplitude holograms were developed using two phase holograms to produce in-plane acoustic images [66]. However, in practice, stationary waves between the two holograms can degrade the image. In this work, we encode both phase and magnitude information into a single phase-only holographic lens. Using this approach, we experimentally, numerically, and theoretically report the generation of zeroth and high-order Bessel beams with elongated field-of-view (of about 40 wavelengths) in the ultrasound regime. 


\subsection{Methods}

\subsubsection{Analytical and numerical field calculations}

We employ a semi-analytical method for theoretical calculations using the Rayleigh-Sommerfeld diffraction integral [see Eq. (2.1) as explained in Chapter 2]. For numerical calculations, we use a pseudo-spectral simulation method with $k$-space dispersion correction to numerically integrate the linearised constitutive relations of acoustics [see Sec. 2.2.2 as explained in Chapter 2]. We use a numerical grid with a spatial step of $\Delta x=\Delta y=\Delta z=221 \mu \mathrm{m}$ and a numerical temporal step of $\Delta t=18.1 \mathrm{~ns}$, leading to a Courant-Friedrichs-Lewy number of 0.13 in water and spatial sampling of 6 grid points per wavelength in water for a frequency of $1.112 \mathrm{MHz}$. These parameters are fixed in all simulations in this chapter.

\subsubsection{Flat-intensity Bessel beams design}

We consider a truncated Bessel beam where the converging conical wavefront is tilted an angle $\beta$ with respect to the normal. If the beam is generated using a source of aperture $2 a$, the non-diffracting beam extends, in an ideal situation, from $z=0 \mathrm{~mm}$ to $z=F=(a / 2) \tan \beta$, as shown in Fig. 6.1 (a). To generate a particular complex wavefront at the source plane $(z=d)$, as the one shown in Fig. 6.1 (c), we employ a holographic phase-plate that can be manufactured using 3D-printing techniques, as shown in Fig. 6.1 (d). These phase plates provide a robust way to engineer the phase-and-amplitude distribution along the surface of the plane [59, 62].

Holographic lenses and fraxicons are 3D-printed using stereolithography (SLA) techniques with a Form 2 printer (Formlabs, USA), with a resolution of $50 \mu \mathrm{m}$ and $100 \mu \mathrm{m}$ in lateral and axial directions, respectively, and using a photosensitive resin (Standard Grey, Formlabs, USA). The acoustical properties of the material were obtained experimentally using a pulse-echo technique in a test cylinder, resulting in a measured sound speed of $c_{L}=2440.7 \mathrm{~m} / \mathrm{s}$ and a density of $\rho_{L}=1162 \mathrm{~kg} / \mathrm{m}^{3}$. The absorption was set to $\alpha=3.06 \mathrm{~dB} / \mathrm{cm}$ at $1.112 \mathrm{MHz}$, matching the reported values of similar polymers [61,62].

The lens surface is divided in squared pixels of different height, $h(x, y)$, 
and uniform width, $\Delta h$, as shown in Fig. 6.1 (d). We assume each elastic column vibrating longitudinally as a Fabry-Perot resonator [for more details, refer to Sec. 2.2.3 in Chapter 2]. The transmission coefficient is close to one if low relative impedance materials are used to build the phase plates, as it is the case of photosensitive polymers used for stereolithographic 3D-printing. In this case, to tailor the complex holographic field (with a particular phase and amplitude as shown in Fig. 6.1 (b)) to an equivalent phase-only field (as shown in Fig. 6.1 (c)), we make use of a direct conversion method to estimate an equivalent holographic lens of uniform field magnitude [for more details, refer to Sec. 2.2.3 in Chapter 2]. The width of the pixels used in this chapter, $2 / 3$ times the wavelength, is thick enough to ensure that the resonance frequency of the first bending mode is far away from the first longitudinal Fabry-Perot resonance frequency.

\subsubsection{Experimental field measurements}

The experiments are conducted inside a $1 \times 0.75 \times 0.5 \mathrm{~m}^{3}$ water tank filled with degassed and distilled water at $22^{\circ} \mathrm{C}$ [as shown in Fig. 6.2]. The ultrasonic transducer is composed of a single element circular piezoceramic crystal (PZT26, Ferroperm Piezoceramics, Denmark) mounted in a custom designed stainless-steel housing with aperture $2 a=50 \mathrm{~mm}$. The transducer was driven with a 100-cycle sinusoidal burst at a frequency of $f=1.112 \mathrm{MHz}$ by a signal generator (PXI5412, National Instruments, USA) and amplified by a linear RF amplifier (ENI 2100, ENI, Rochester, NY). The pressure field is measured by a calibrated needle hydrophone with a $500 \mu \mathrm{m}$ active diameter (HNR-500, Onda). The hydrophone signals are acquired by a digitizer (PXI5620, National Instruments, USA) and averaged 100 times to increase the signal-to-noise ratio. A 3D micropositioning system (OWIS GmbH) is used to move the hydrophone in three orthogonal directions with an accuracy of $10 \mu \mathrm{m}$. All the signal generation and acquisition processes are based on a NI8176 National Instruments PXI-Technology controller, which also controls the micro-positioning system. Temperature measurements are performed throughout the whole process to ensure no temperature changes of $\pm 0.5^{\circ} \mathrm{C}$. The transverse maps were acquired in the range $(x, y)= \pm 3 \mathrm{~mm}$ using a step of $0.15 \mathrm{~mm}$, the transverse cross-section lines were acquired in the range $x= \pm 7 \mathrm{~mm}$ 


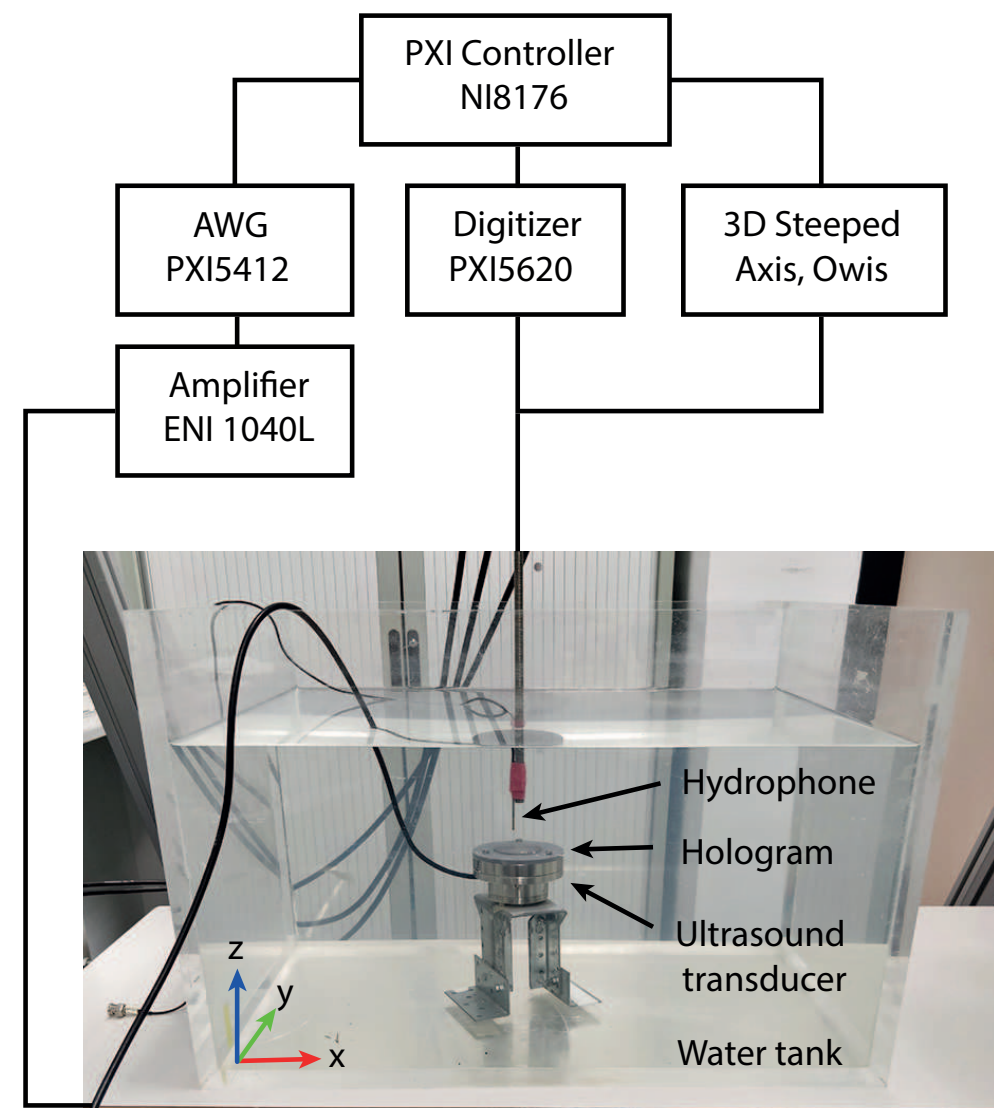

Figure 6.2: Diagram of the experimental set-up and photograph of one experiment in water tank.

using a step of $0.1 \mathrm{~mm}$, and the axial measurements were acquired from 10 to $45 \mathrm{~mm}$ using a step of $1 \mathrm{~mm}$.

\subsection{Generating a Bessel beam}

The initial conditions corresponding to common approaches to produce zeroth-order Bessel beams are shown in Fig. 6.3 (a1-c1). First, we show the Bessel beam produced by a binary-amplitude grating [40], which is 

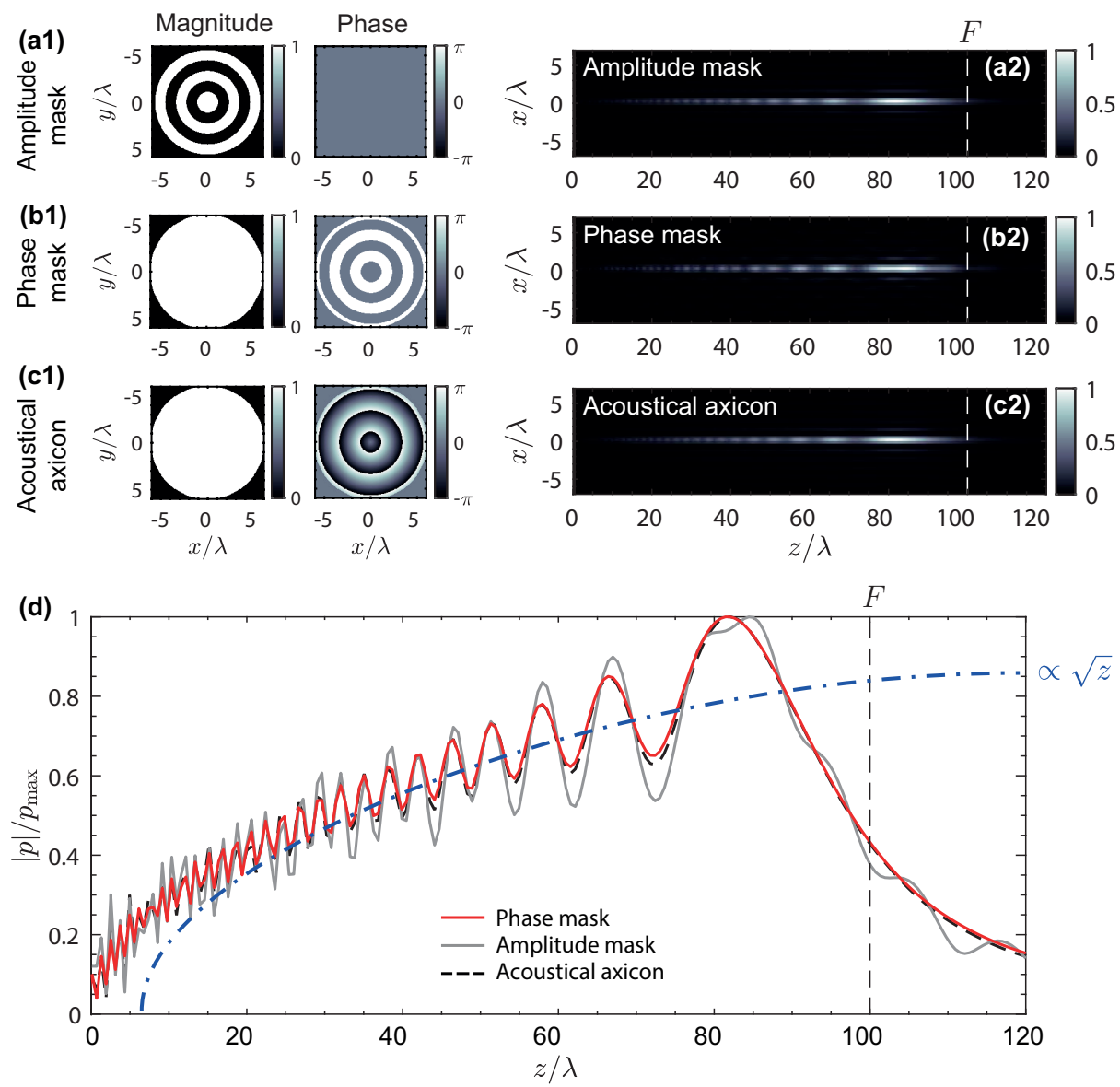

Figure 6.3: Different theoretical methods to generate a non-flat-axial-intensity Bessel beam. (a1) Magnitude and phase distribution at the source of an axisymmetric diffraction grating (amplitude mask type) and (a2) its axial pressure distribution (normalized). (b1) Magnitude and phase distribution at the source of an axisymmetric diffraction grating (phase mask type) and (b2) its axial pressure distribution. (c1) Magnitude and phase distribution at the source of an acoustical fraxicon and (c2) its axial pressure field distribution. (d) Axial pressure for the non-flat axial intensity Bessel beams. 
equivalent to a Soret-type Zone Plate lens [67]. This lens comprises alternating axisymmetric opaque and transparent zones separated at a distance b, as shown in Fig. 6.3 (a1). Due to the continuity of the wave-vector at the interface, the propagating wavefront presents a phase distribution of

$$
p_{0}(r)=\exp \left(i k_{r} r\right)
$$

where $k_{r}=\sin (\beta) k_{0}$ is the transverse wave-vector, fixed by the lens, $k_{0}=\omega / c_{0}$ the wavenumber and $\beta=\sin ^{-1}\left(2 \pi / k_{0} b\right)$ is the angle of the tilted wavefront of the Bessel beam. While a Bessel beam is generated [40] its axial field distribution is not constant, as shown in Fig. 6.3 (a2). As the total surface of each annular area varies with the radial coordinate, the intensity radiated by each annulus depends linearly on the distance to the center. Therefore, the intensity pattern grows linearly with distance (and proportional with the square root of the axial distance, see Fig. 6.3 (d)). An analogous situation occurs when the lens is an alternating phase-inversion axisymmetric grating, as shown in Fig. 6.3 (b1). This can be obtained by using a phase plate where the thicknesses vary between 2 values, $L=[(2 m-1) \lambda / 2, m \lambda]$ with $m \in \mathbb{N}$. In this case the resulting beam intensity also grows linearly with the axial distance as shown Fig. 6.3 (b2). Equivalently for axisymmetric gratings, acoustical axicons (cone-shaped lenses that have been proposed for optics and acoustics) can also produce a converging conical wavefront due to refraction on its tilted surface. A variation of conical axicon lenses are fraxicon lenses. Fraxicons, in analogy to Fresnel lenses, present a wrapped profile able to produce a similarly tilted wavefront by the refraction along its inclined surface. The phase profile of a fraxicon varies linearly with the radial coordinated as $p_{0}(r)=\exp \left(i k_{r} r\right)$, as shown in Fig. 6.3 (c1). However, the exterior concentric annular areas radiate more energy than the interior ones as they present more surface, as occurs with the axisymmetric gratings. In this way, the corresponding axial field distribution is not constant either, as shown in Fig. 6.3 (d).

In contrast, the field at the source plane of an ideal (truncated) Bessel beam given by

$$
p_{0}(r)=J_{n}\left(k_{r} r\right),
$$

where $J_{n}$ is the $n$-th order Bessel beam, is shown in Fig. 6.4 (a1). The resulting field distribution is shown in Fig. 6.4 (a2,d), showing an almost 

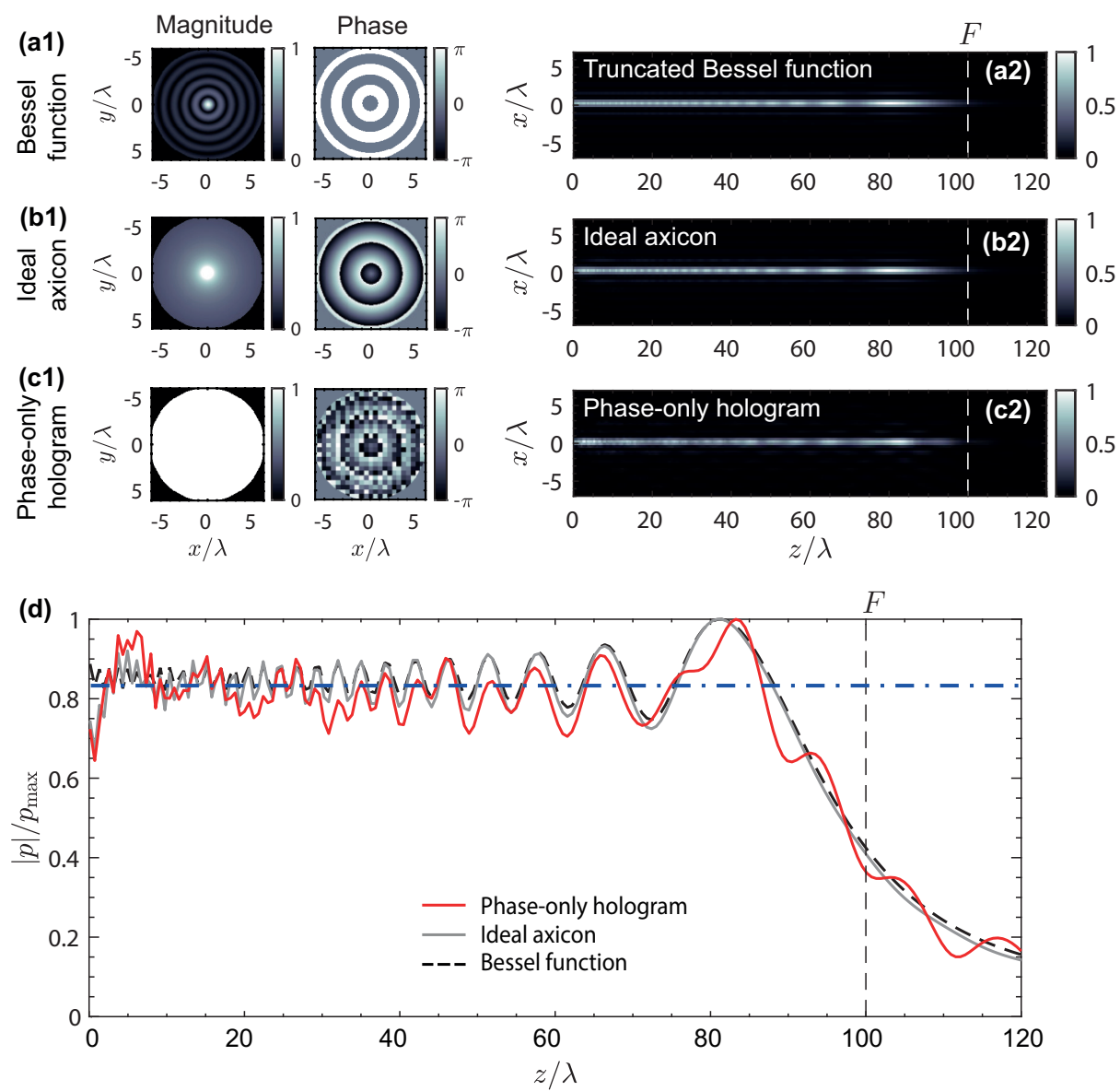

Figure 6.4: Different theoretical methods to generate a flat-axial-intensity Bessel beam. (a1) Magnitude and phase distribution at the source of an exact truncated Bessel function and (a2) its axial pressure distribution (normalized). (b1) Magnitude and phase distribution at the source of an ideal axicon and (b2) its axial pressure distribution. (c1) Magnitude and phase distribution at the source of a phase-only acoustical hologram lens encoded with the ideal axicon and (c2) its axial pressure distribution. (d) Axial pressure for the flat-axial-intensity Bessel beams. 
flat profile. Here, the beam extends from $z=0 \mathrm{~mm}$ to $z=F$, a total distance of $100 \lambda$. The existing ripples are caused by the limited aperture of the source. Note that the source pattern presents both magnitude and phase variations, which are of the order of the wavelength. It is worth noting here that to our knowledge, the source condition corresponding to Eq. 6.2 has only been reproduced using active methods using a finite set of concentric rings.

In order to reduce the complexity of the Bessel pattern one can design an equivalent field distribution using an ideal axicon, i.e., imposing a compensation for the intensity as a function of the radial coordinate. By simple geometrical considerations, this results in a source field of

$$
p_{0}(r)=\frac{1}{\sqrt{\left.\max \left(r, r_{0}\right)\right)}} \exp \left(i k_{r} r\right)
$$

where $r_{0}$ is set to a finite value to avoid the singularity, e.g., the pixel size, as shown in Fig. 6.4 (b1). This field distribution also produces a Bessel beam of constant amplitude analogously to that of the ideal-truncated Bessel function, as demonstrated in Fig. 6.4 (b2,d). Note that the ripples match those of the ideal-truncated Bessel function. However, the source field for the ideal axicon presents a reduced spatial complexity compared with the ideal Bessel function. This makes it more suitable to apply a direct method to obtain an equivalent phase-only lens. Once applying the direct method [68], the resulting phase-only hologram is shown in Fig. 6.4 (c1), using a pixel size of $\lambda / 8$. The field generated by this lens is shown in Fig. $6.4(\mathrm{c} 2, \mathrm{~d})$. We can observe that the field distribution is flat and the characteristic ripples of the Bessel beam obtained by using the hologram agree with those of the finite-aperture Bessel beam.

\subsection{Performance of holographic Bessel beams}

Diffraction effects of any beam are stronger for low frequencies, e.g., when the ratio $a / \lambda$ is small. Thus, the capability of any finite-aperture Bessel beam to concentrate the acoustic energy over the axis will also be limited by the natural diffraction of the beam. We have designed 100 holograms for frequencies ranging from $100 \mathrm{kHz}$ to $10 \mathrm{MHz}$ to study the effect of the frequency in their focusing performance. The aperture of the source and 
the focal length of the lens were maintained constant $(a=25 \mathrm{~mm}$ and $F=50 \mathrm{~mm}$ ). Figs. $6.5(\mathrm{a}, \mathrm{b})$ show the axial field distribution for the ideal truncated Bessel beam and the corresponding Bessel beam generated by the phase-only hologram. First, we can observe that both calculations agree, showing that the proposed method can be used in a broad range of frequencies. In particular, Figs. 6.5 (c,d,e) show the axial distribution for $0.5 \mathrm{MHz}, 2 \mathrm{MHz}$ and $5 \mathrm{MHz}$. These frequencies correspond to $a / \lambda \approx$ $(8,33,83)$, respectively. We can observe that the spatial distribution shows a flatter profile for higher frequencies, and the energy corresponding to both ideal and holographic Bessel beams is evenly distributed along the axis. For lower frequencies, e.g., at $f=0.5 \mathrm{MHz}$, both beams suffer from stronger diffraction effects and, therefore, the energy of the beam only extends to a fraction of the focal length (up to $z \approx 0.8 F$ in this particular case). In addition, at lower frequencies, the amplitude of the ripples is increased as compared with the ones corresponding to higher frequencies. Note that this behavior is not a limitation of the method using holograms, it is caused by the natural diffraction of the wavefront and it will be observed in any truncated Bessel beam propagating in homogeneous and linear media.

\subsection{Broadband behaviour of fraxicons and phase- only holograms}

In the previous section we have shown how fraxicons and holograms perform at the design frequency. In this section, we design a fraxicon and a hologram for a particular frequency and then evaluate their performance for other excitation frequencies. We might notice that the field distributions at the source for both fraxicon and ideal Bessel beams present spatial modulations in the radial direction characterized by the distance $b=2 \pi / k_{r}$, with the transverse wavenumber fixed by $k_{r}=k_{0} \sin (\beta)$ (see, e.g., the concentric rings separated at a distance $b$ that appears at the source distributions shown in Fig. 6.3 and Fig. 6.4). When a wave impinges these lenses, its phase and amplitude are modified according to the local thickness of the lens. For frequencies much lower than the design frequency $(\lambda>b)$, a small phase change is produced because the maximum height of the fraxicon lens is much lower than the wavelength. Therefore, 

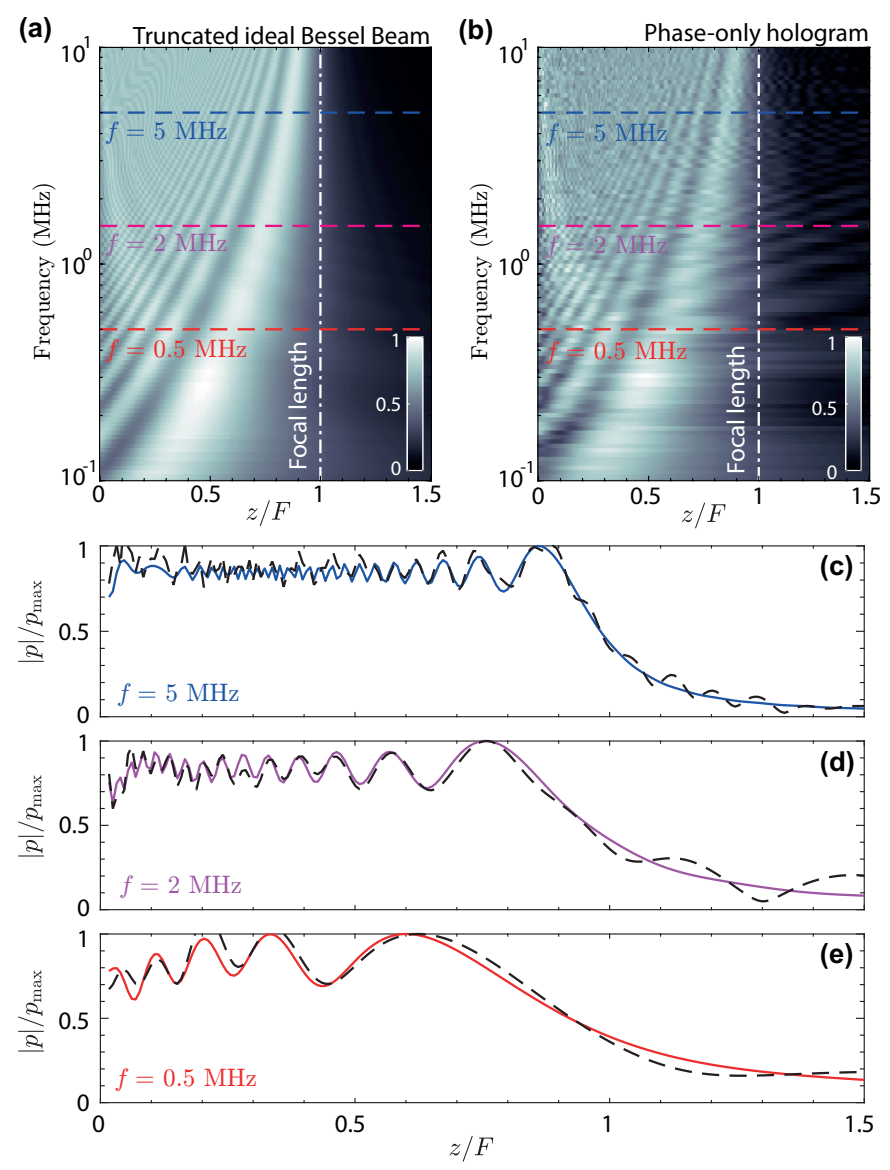

Figure 6.5: Axial field distribution as a function of the design frequency for (a) a truncated-ideal Bessel beam and (b) a phase-only hologram calculated using the Rayleigh-Sommerfeld integral. (c) Axial field distribution for a Bessel beam of $f=5 \mathrm{MHz}$ (blue) and the corresponding field produced by the phase-only hologram (dashed). (d) Axial field distribution for a Bessel beam of $f=2 \mathrm{MHz}$ (pink) and the corresponding field produced by the phase-only hologram (dashed). (e) Axial field distribution for a Bessel beam of $f=500 \mathrm{kHz}$ (red) and the corresponding field produced by the phase-only hologram (dashed). 
the field is transmitted, and the field of a piston-like source is retrieved. However, for frequencies lower than the design frequency but higher than $\lambda \leq b$, waves are diffracted due to the spatial modulation of the lens, even when the transmitted phase modulation is small. In this situation, fraxicons and holograms act similarly to axisymmetric diffraction gratings [40].

Due to the conservation of the transverse wavenumber at the boundary, waves are diffracted at angles given by Eq. 6.4 producing a tilted conical wavefront for each diffraction order $n$. In consequence, the extent of the Bessel beam changes with the excitation frequency.

$$
\beta_{n}(\omega)=\sin ^{-1}(n \lambda / b)
$$

By simple trigonometric relations we can obtain the focal length for each diffraction order as

$$
F_{n}(\lambda)=\frac{a b}{n \lambda} \sqrt{1-\left(\frac{n \lambda}{b}\right)^{2}}
$$

Note that for $\lambda>b$ the focal distance becomes imaginary for all diffraction orders. This implies a lower cut-off frequency for the fraxicons and phase-only holograms, $f_{\text {low }}$, which is fixed by the lens design as

$$
f_{\text {low }}=\frac{c_{0}}{b}=f_{0} \sin (\beta)=f_{0} \frac{a}{\sqrt{a^{2}+F^{2}}},
$$

Below this frequency phase-only holograms and fraxicons whose maximum height corresponds to $\lambda$ cannot produce any Bessel beam. Fig. 6.6 shows the axial field distribution of a zeroth-order Bessel beam as a function of the frequency for a fraxicon [Fig. 6.6 (a)] and phase-only hologram [Fig. $6.6(\mathrm{~b})$ ] with the design parameters $F=50 \mathrm{~mm}, a=25 \mathrm{~mm}$ and $f_{0}=1.112 \mathrm{MHz}$. This results in a $b=3 \mathrm{~mm}$ ring distance, which imposes the lower cut-off frequency of $f_{\text {low }}=497.3 \mathrm{kHz}$. The focus of the $n$-th diffraction order, $F_{n}$, is shown in dotted-dashed white lines. We can see that the focal length is extended from $z=0 \mathrm{~mm}$ to $z=F_{n}$, with $n=1$. Some energy is also transmitted for frequencies below $\lambda>b$, corresponding to the zeroth diffraction order. For frequencies around $\lambda \approx b$, the focal length of the first diffraction order is close to the source (e.g., $F_{1}=8 \mathrm{~mm}$ 
(a)
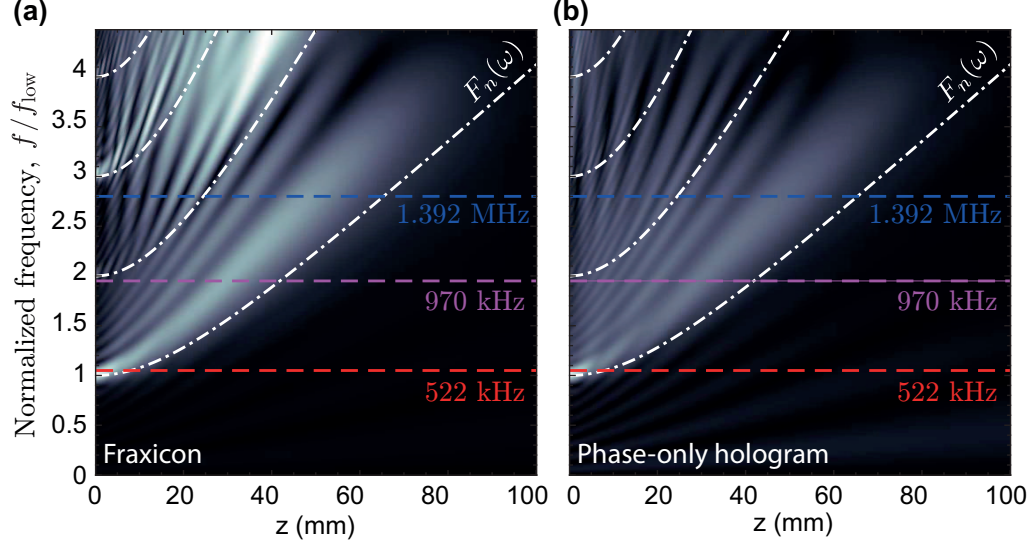

(c)

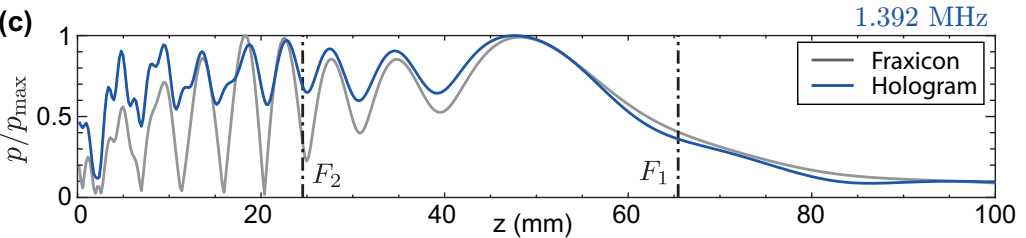

(d)
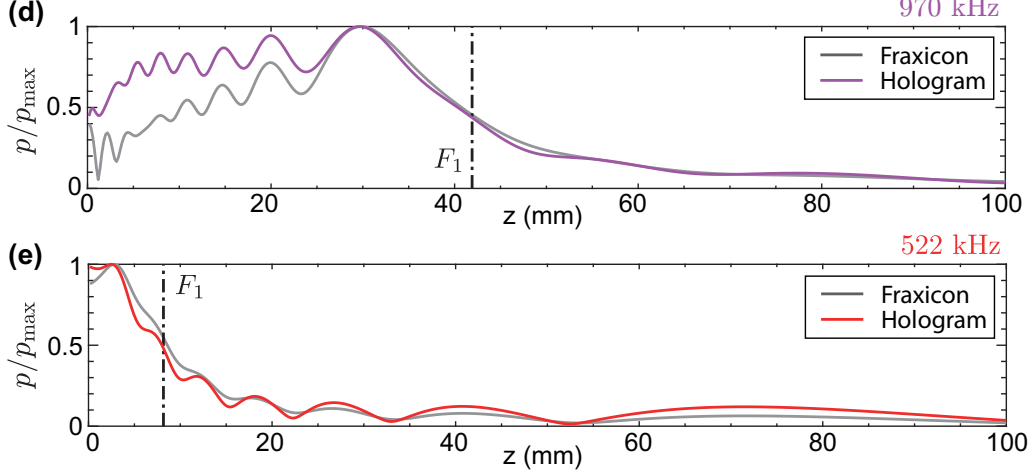

Figure 6.6: Axial field distribution for a zeroth-order Bessel beam as a function of the excitation frequency of (a) a fraxicon and (b) phase-only hologram of $F=50 \mathrm{~mm}, a=25 \mathrm{~mm}$ and for a design frequency of $f_{0}=1.112 \mathrm{MHz}$. The focus of the $n$-th diffraction order, $F_{n}$, is shown in dotted-dashed white lines. (c-e) Axial field distributions at $1.392 \mathrm{MHz}$, $970 \mathrm{kHz}$ and $522 \mathrm{kHz}$, respectively. The focus of the $n$-th diffraction order, $F_{n}$, is shown in dotted-dashed black lines. 
at $522 \mathrm{kHz}$ ). Therefore, the field is strongly focused at this distance, as shown in Fig. 6.6 (e). As the phase-only hologram maintains a similar spatial modulation with characteristic rings separated at a distance $b$, a similar focusing pattern is observed, as shown in Fig. 6.6 (b,e). For higher frequencies, e.g., at $970 \mathrm{kHz}$, the focus is extended to $F_{1}=41.9 \mathrm{~mm}$. Accordingly, the field distributes up to this distance. On the one hand, the axial field distribution of the fraxicon is distributed with a square root dependence of the distance (its intensity is linear with the distance). On the other hand, the hologram produces a uniform field distribution, as shown in Fig. 6.6 (d). Finally, for higher frequencies, high-order diffraction orders appear, each one presenting a different focal length given by Eq. 6.5. Each diffraction order produces a new Bessel beam that overlaps with the preceding one. An example is given in Fig. 6.6 (c) for $f=1.392 \mathrm{MHz}$. The field produced by the first diffraction order extends up to $F_{1}=65.4 \mathrm{~mm}$, while the focus of the second diffraction order appears at $F_{2}=24.5 \mathrm{~mm}$. The coherent sum of the two fields produces an interference pattern with strong axial variations between $z=0 \mathrm{~mm}$ and $z=F_{2}$. Overall, the phase-only hologram produces a flatter beam because error-diffusion algorithm smoothes the spatial modulations corresponding to rings separated at a distance $b$.

\subsection{Pixel quantization}

In standard stereolithographic 3D-printing techniques, the lenses are manufactured using layers of finite thickness. In addition, in simulations, the pixels are represented with a discrete set of heights. In particular, in the simulations included in this chapter, the pixels are discretized in $221 \mu \mathrm{m}$ steps (the grid resolution) and for the experiments in $100 \mu \mathrm{m}$ steps (the printer resolution). The corresponding phase distributions of the fraxicons and holograms are therefore quantized in discrete steps.

To test the impact of the height quantization, we have designed a set of fraxicons and holograms using different quantization steps $\left(\Delta_{d}\right)$, ranging from $\Delta_{d}=1 \mu \mathrm{m}$ to $\Delta_{d}=1.3 \mathrm{~mm}$, as shown in Figures 6.7 and 6.8. The phase is calculated using the Fabry-Perot equation [Eq. (2.6)] and the acoustic field is obtained using the Rayleigh-Sommerfeld equation [Eq. (2.1)]. Several fraxicons are designed using different quantization 

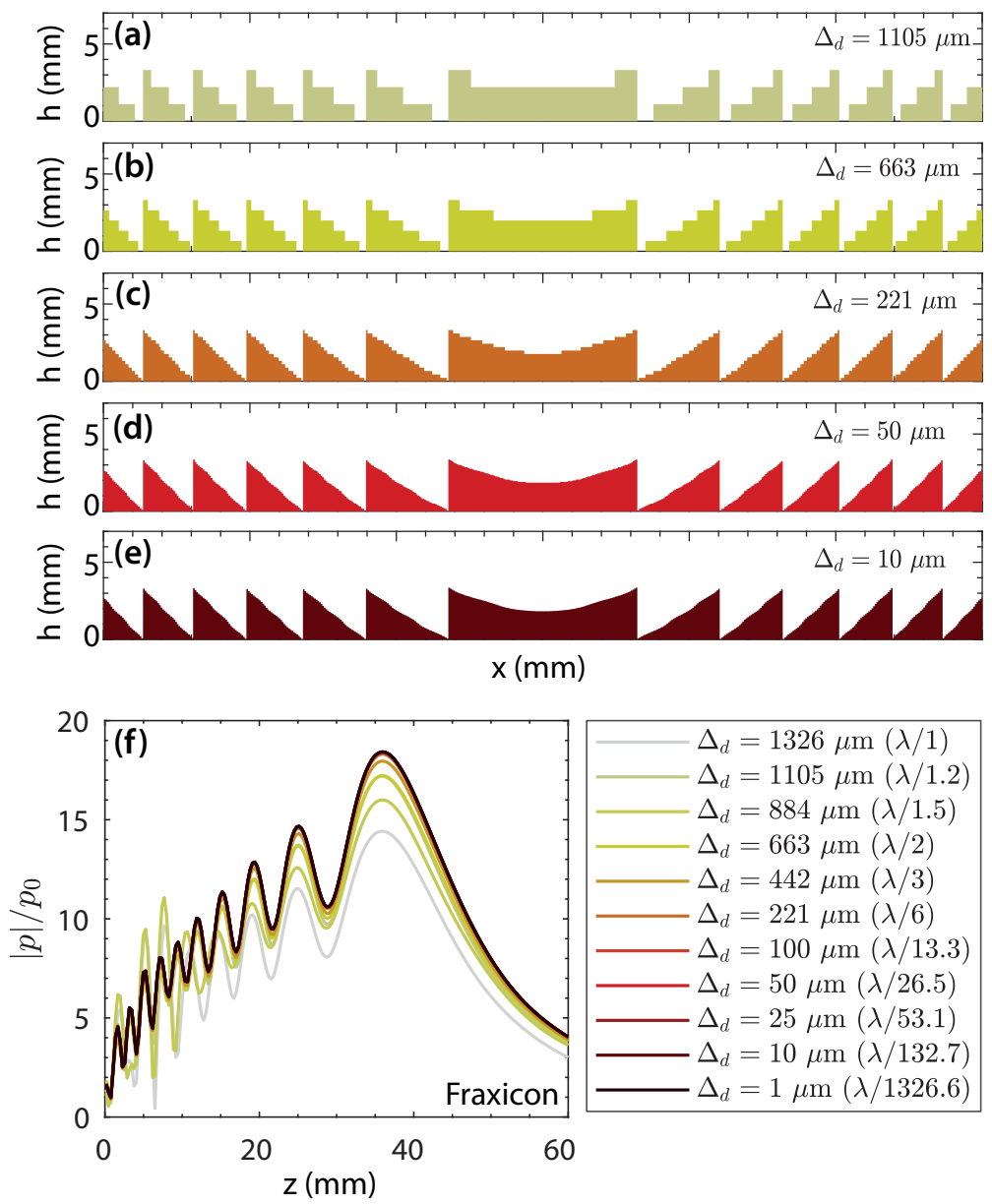

Figure 6.7: (a-e) Transversal cut of the fraxicons using different discretization heights. (f) Corresponding theoretical field for the fraxicons using different discretization heights.

levels, as shown in Fig. 6.7 (a-e). The axial field for each fraxicon is shown in Fig. 6.7 (f). First, the shape of the pressure distribution for all the fraxicons roughly agrees with the one corresponding to the lower 

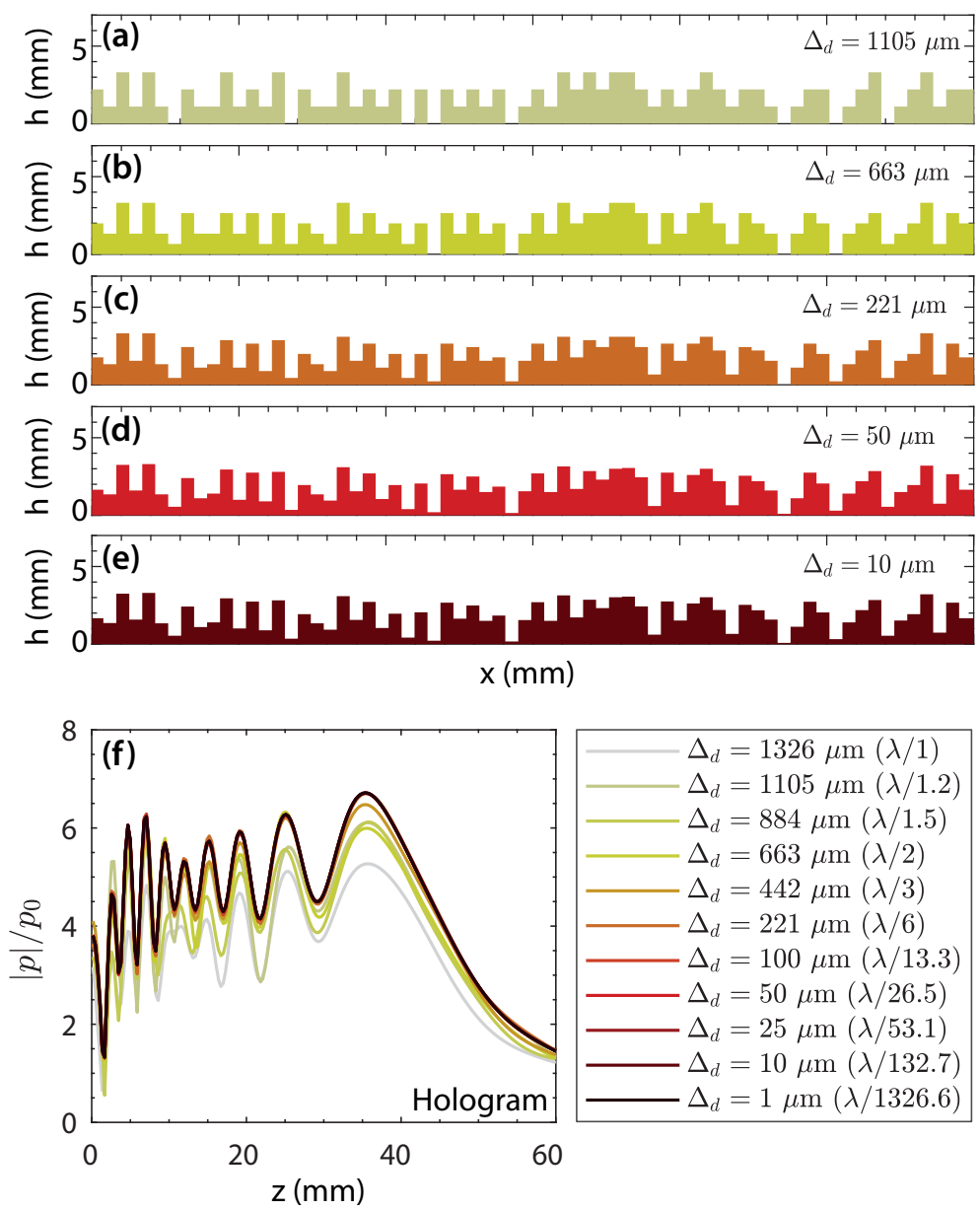

Figure 6.8: (a-e) Transversal cut of the phase-only holograms using different discretization heights. (f) Corresponding theoretical field for the phase-only holograms using different discretization heights.

quantitation level. Even when only 6 values are available for the pixel height $\left(\Delta_{d} \approx 663 \mu \mathrm{m}\right)$, the steeped lenses produce similar fields. Note that using only two values for the height corresponds to an axisymmetric 
phase grating, which can also produce a Bessel beam. One remarkable difference is that when using coarser quantization levels, the amplitude of the field is reduced. This is caused because energy is diffracted into higher diffraction orders, causing secondary focal spots near the source. The quantization level used in the simulations $\left(\Delta_{d}=221 \mu \mathrm{m}\right)$ produces a field in excellent agreement with the one corresponding to $\Delta_{d}=1 \mu \mathrm{m}$, showing that the accuracy of the simulated (and manufactured) lenses is enough to produce Bessel beams with flat pressure distributions.

The same study was extended to phase-only holograms using different quantization levels, as shown in Fig. 6.8 (a-e). The corresponding axial field for each phase-only hologram is shown in Fig. 6.8 (f). We observe that phase-only holograms using low quantization levels, e.g., $\Delta_{d}<100 \mu \mathrm{m}$, can produce fields in close agreement with the one corresponding to $\Delta_{d}=$ $1 \mu \mathrm{m}$. Moreover, quantization levels of only $\Delta_{d} \approx \lambda / 3$ are enough to produce fields in agreement with the truncated-Bessel beam distributions. The main difference is that, as observed previously in fraxicons, at lower quantization levels, energy can be diffracted into higher diffraction orders and, therefore, the gain of the lens $\left(\left|p_{\max }\right| / p_{0}\right)$ is reduced.

Finally, it is worth to mention here that the gain of fraxicon lenses is higher (about 2.74 times) than the gain of phase-only lenses. The reduction of the gain is caused because holograms produced by the error-diffusion algorithm mimic the phase-and-magnitude distribution of ideal Bessel beams, which presents lower amplitude at the external areas of the lens [see Fig. 6.4 (c1)]. This gain reduction in phase-only lenses is also due to the energy spreading along the axial direction instead of concentrating the whole constructive interference at the focal spot as fraxicons do.

\subsection{Experimental validation}

We performed an experimental test in the ultrasonic regime using a phase-only hologram of aperture $2 a=50 \mathrm{~mm}$ and a depth-of-field $F=50 \mathrm{~mm}$ $(\approx 40 \lambda)$ using a source of $1.11 \mathrm{MHz}$ in water. In addition, a full-wave simulation was performed using a time-domain pseudo-spectral method with $k$-space correction. A fraxicon also was manufactured for comparison. Both zeroth and first-order Bessel beams were considered. The 3D-printed lenses are shown in Fig. 6.9. Details about the measurement procedures 

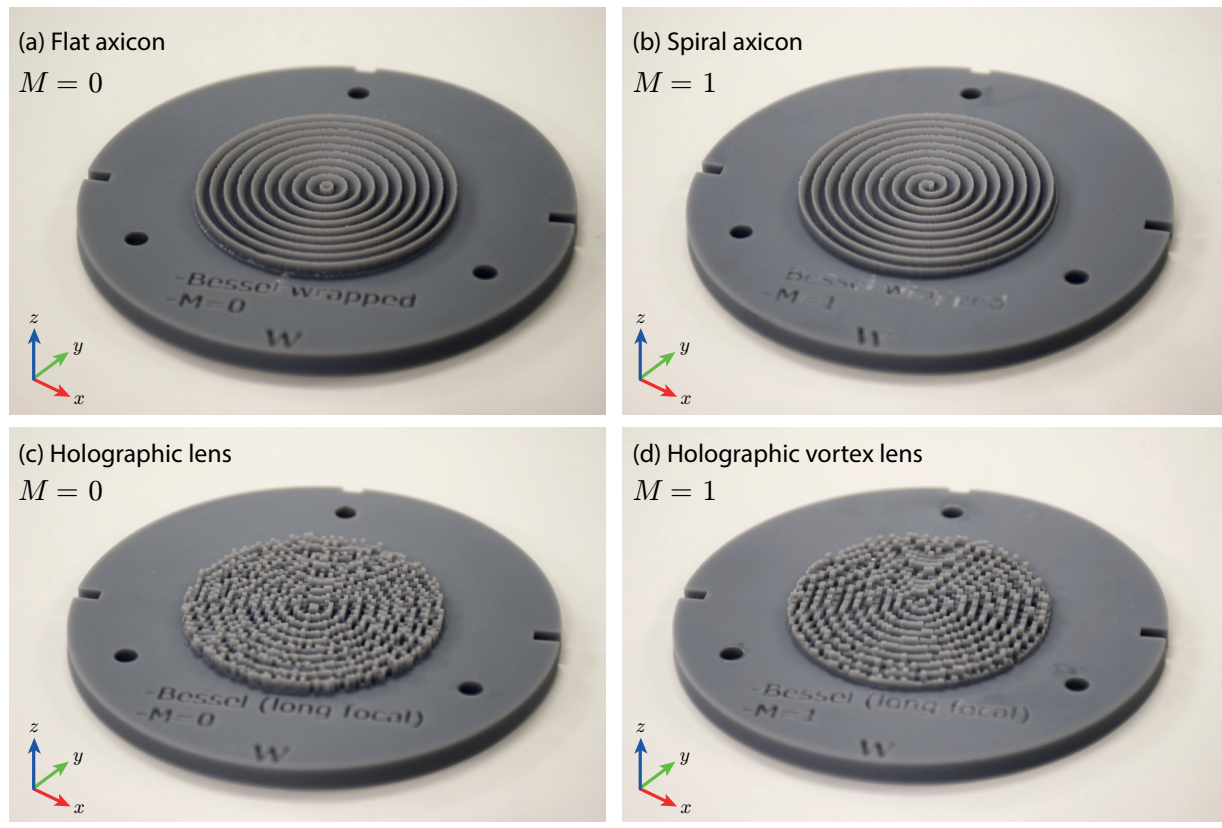

Figure 6.9: Manufactured lenses by 3D-printing. (a) Fraxicon for a zeroth-order Bessel beam $(M=0)$. (b) Spiral fraxicon for a first-order Bessel beam $(M=1)$. (c-d) Phase-only holographic lenses for flat-intensity Bessel beams corresponding to $M=0$ and $M=1$ (vortex).

and simulation methods can be found in Sec. 6.2.3.

\subsubsection{Zeroth-order Bessel beams}

The simulated field distribution of the fraxicon is shown in Fig. 6.10 (a1), showing a non-flat field distribution as discussed theoretically earlier. The theoretical, numerical, and experimental magnitude and phase transversal field distributions are shown in Figs. 6.10 (b1,c1,d1,e1,f1,g1). We can see that the magnitude of the field shows a good symmetry in the three cases, while the phase remains radially constant. The simulated and experimental axial field distributions, shown in Fig. 6.10 (h1), agree with that obtained by theory. While the transversal field distribution measured at 

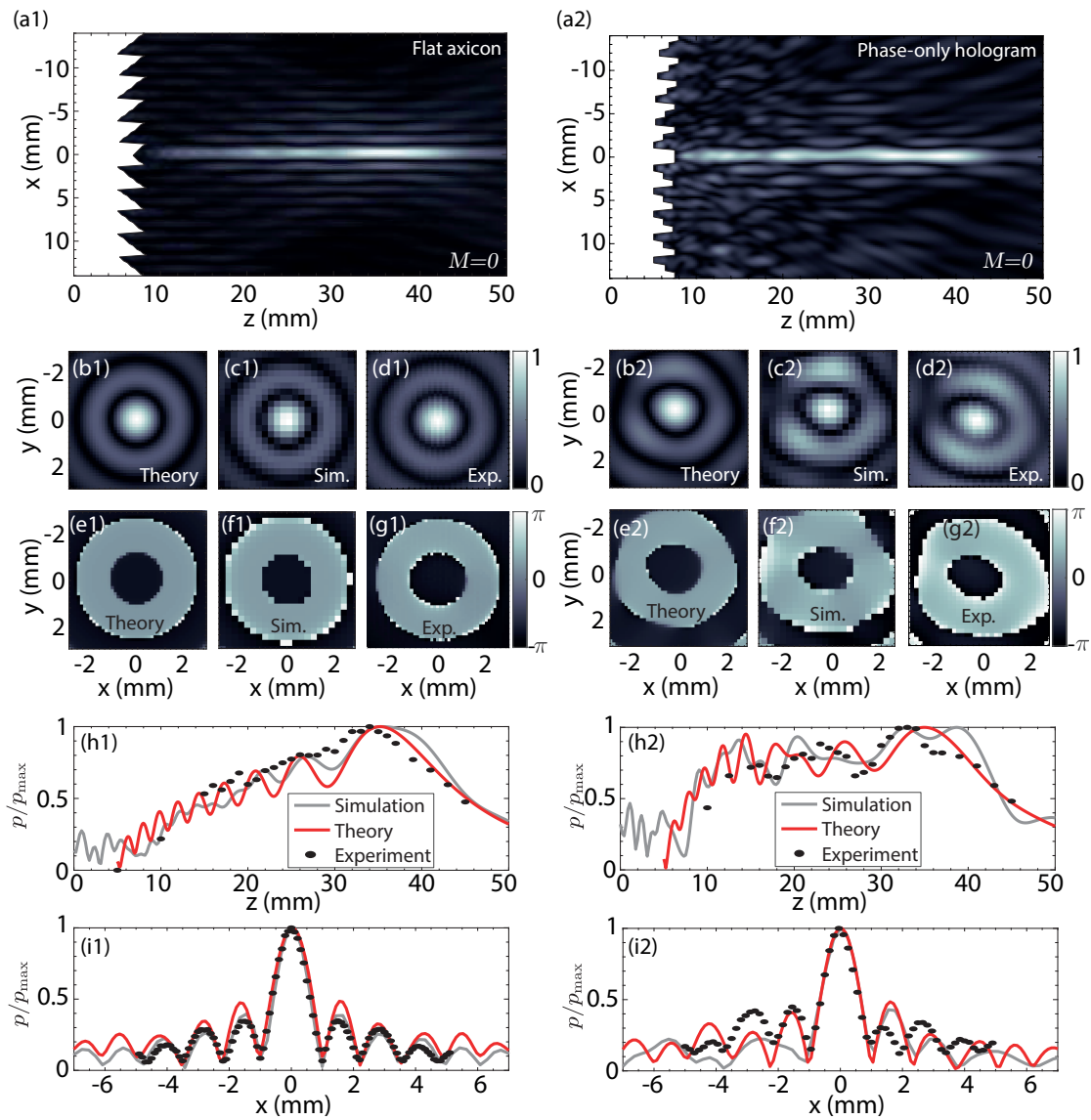

Figure 6.10: Results for the zeroth-order Bessel beams. Field distribution in the sagittal plane for the fraxicon lens (a1) and the phase-only hologram (a2). Field magnitude in the transverse direction (at $z=35 \mathrm{~mm}$ ) obtained for the fraxicon using theory (b1), simulations (c1) and experiments (d1), and obtained for the phase-only hologram using theory (b2), simulations (c2) and experiments (d2). Corresponding theoretical, numerical and experimental phase of the field in the transversal direction (at $z=35 \mathrm{~mm}$ ) for the fraxicon $(\mathrm{e} 1, \mathrm{f} 1, \mathrm{~g} 1)$ and the phase-only hologram $(\mathrm{e} 2, \mathrm{f} 2, \mathrm{~g} 2)$. Axial and transversal (at $z=35 \mathrm{~mm}$ ) field cross-sections for the fraxicon (h1,i1), respectively, and for the phase-only hologram $(\mathrm{h} 2, \mathrm{i} 2)$. 
$z=35 \mathrm{~mm}$ for the fraxicon matches that of a truncated Bessel beam [see Fig. 6.10 (i1)], the axial field distribution grows proportionally to the square root of the axial distance. The corresponding simulated field of the phase-only hologram is shown in Fig. 6.10 (a2). We can observe that the field distribution is remarkably uniform as compared with the fraxicon. The theoretical, numerical, and experimental magnitude and phase transversal field distributions are shown in Figs. 6.10 (b2,c2,d2,e2,f2,g2). Again, we can see that the magnitude of the field shows a good symmetry in the three cases, while the phase remains radially constant. The axial field distribution is shown in Fig. 6.10 (h2) where, again, simulated and experimental data are in reasonable agreement with theory. Some discrepancies are observed due to the finite size of the pixel used, which is restricted by the 3D-printing technology (the lateral resolution of the printer is $50 \mu \mathrm{m})$.

\subsubsection{High-order Bessel beams (vortex beams)}

Beyond zeroth-order, Bessel beams of higher order containing phase dislocations can also be generated by the proposed technique. We manufacture a phase-only hologram for a first-order Bessel beam using a pressure distribution of

$$
p_{0}(r, \theta)=\frac{1}{\sqrt{\left.\max \left(r, r_{0}\right)\right)}} \exp \left(i k_{r} r\right) \exp (i M \theta)
$$

where the topological charge of a first-order Bessel beam corresponds to $M=1$ and $\theta$ is the polar angle. A spiral fraxicon, corresponding to a blazed-vortex axicon, is also manufactured for comparison using a source profile of

$$
p_{0}(r, \theta)=\exp \left(i k_{r} r\right) \exp (i M \theta),
$$

Fig. 6.11 (a1) shows the simulated field for the spiral fraxicon while Fig. 6.11 (a2) shows the corresponding one for the phase-only hologram. As occurs with the zeroth-order Bessel beam, our approach results in a flatter beam profile. The hollow beam is reproduced by all methods with the characteristic null at the center. This null arises because of the phase dislocation of the vortex. The theoretical, numerical, and experimental magnitude and phase transversal field distributions are shown in 

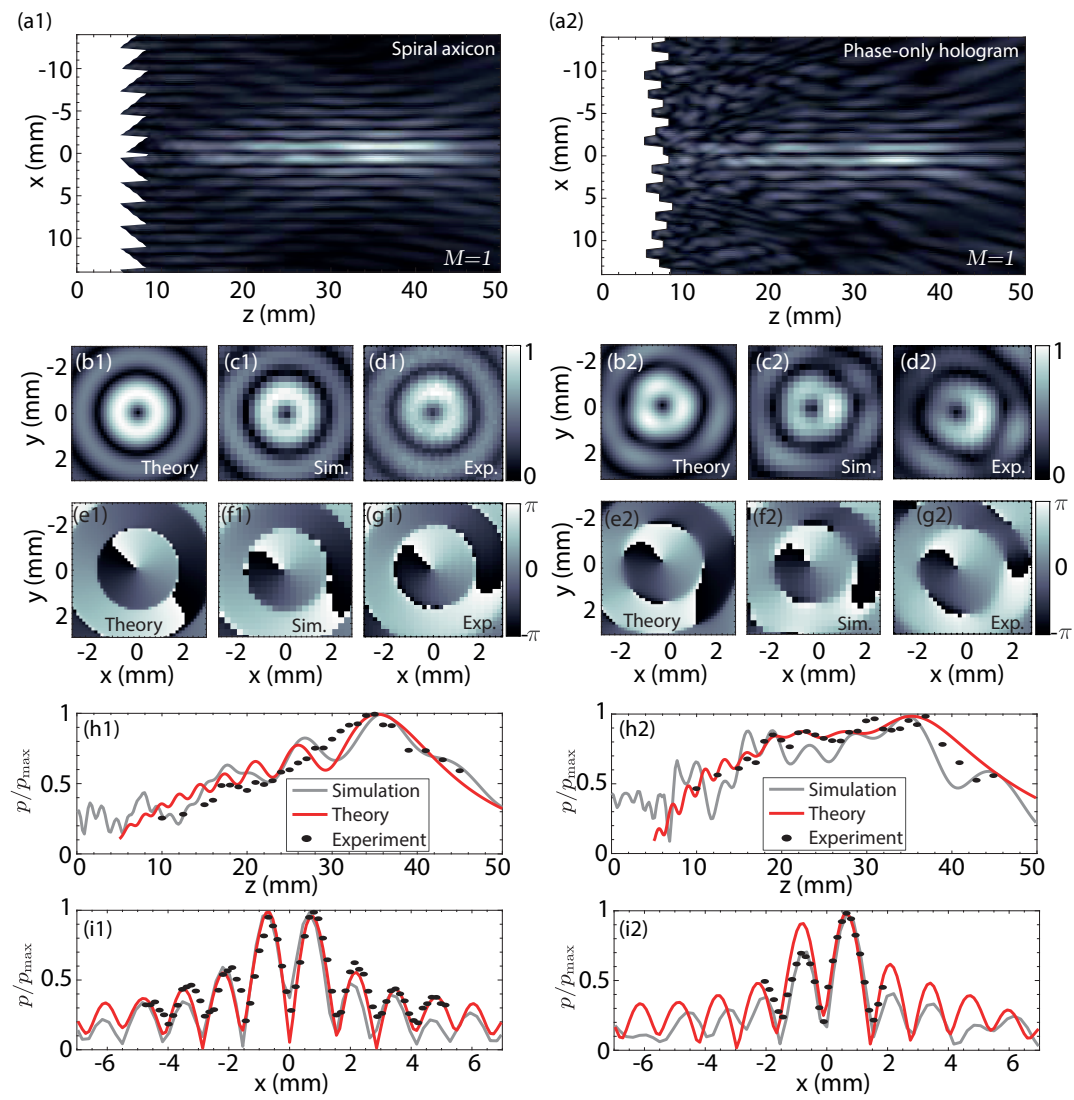

Figure 6.11: Results for the first order Bessel beams, i.e., vortex beams. Field distribution in the sagittal plane for the spiral fraxicon lens (a1) and the phase-only vortex hologram (a2). Field magnitude in the transverse direction (at $z=35 \mathrm{~mm}$ ) obtained for the spiral fraxicon using theory (b1), simulations (c1) and experiments (d1), and obtained for the phase-only hologram using theory (b2), simulations (c2) and experiments (d2). Corresponding theoretical, numerical and experimental phase of the field in the transversal direction (at $z=35 \mathrm{~mm}$ ) for the spiral fraxicon $(\mathrm{e} 1, \mathrm{f} 1, \mathrm{~g} 1)$ and the phase-only hologram $(\mathrm{e} 2, \mathrm{f} 2, \mathrm{~g} 2)$. Axial and transversal (at $z=35 \mathrm{~mm}$ ) field cross-sections for the spiral fraxicon $(\mathrm{h} 1, \mathrm{i1})$, respectively, and for the phase-only hologram $(\mathrm{h} 2, \mathrm{i} 2)$. 
Figs. 6.11 (b1,c1,d1,e1,f1,g1) and $6.11(\mathrm{~b} 2, \mathrm{c} 2, \mathrm{~d} 2, \mathrm{e} 2, \mathfrak{f 2}, \mathrm{g} 2)$, respectively for spiral fraxicon and phase-only hologram. We can see that the magnitude of the field shows a good symmetry in the three cases, while a phase proportional to $\exp (i M \theta)$ is reproduced. In all cases, the topological charge of the vortex is the unity $(M=1)$, while at $r=0$, a phase dislocation is observed. The corresponding axial and lateral cross-sections are given in Fig. 6.11 (h1,i1,h2,i2), respectively. The measured and simulated pressure distributions agree with the theoretical ones.

\subsection{Conclusions}

We have presented a simple method to generate Bessel beams of flat intensity by using acoustic holograms. The magnitude and phase distribution of an ideal axicon was transformed into an equivalent phase-only hologram applying a direct method and used to manufacture an ultrasonic lens by using 3D-printing. We demonstrated the approach theoretically, and validated it by using full-wave simulations and experiments. In particular, a zeroth-order Bessel beam was first produced in the ultrasonic regime. The beam evenly distributes the acoustic energy over a flat line along an axial distance of $50 \mathrm{~mm}(\approx 40 \lambda)$ while the width of the beam remained constant at about $1 \mathrm{~mm}(\approx 0.7 \lambda)$. Using the proposed approach, the generated beam patterns match those of an ideal (truncated) Bessel beam. Note that, in contrast, traditional methods using passive ultrasonic devices produce non-uniform beam patterns.

Beyond zeroth-order beams, we have demonstrated that higher-order Bessel beams can also be generated. A first-order Bessel beam was obtained, where the characteristic vortex containing a phase dislocation was observed experimentally in excellent agreement with theory and full-wave simulations. It is worth noting here that, in contrast with zeroth-order Bessel beams, higher-order Bessel beams cannot be generated by using radially symmetric active systems: active transducers with chiral symmetry are required making this approach a robust solution to generate flat-intensity beams with broad depth-of-field. We remark that in contrast to Bessel beams produced by computer-generated holograms in optics $[69,70]$, the axial intensity of the beam using the present approach is flat. 
The proposed method opens the pathway to future investigations of the singular properties of Bessel beams, including their nonlinear propagation features [18, 71, 72], angular momentum transfer [18], and particle manipulation capabilities [15, 19, 73-77]. These beams may find potential uses in particle manipulation and acoustic radiation force techniques, ultrasound imaging, or therapeutic ultrasound applications.

\section{References - Chapter 6}

[1] J. Durnin, "Exact solutions for nondiffracting beams. i. the scalar theory," J. Opt. Soc. Am. A, vol. 4, p. 651, 1987.

[2] J. Durnin, J. Miceli Jr, and J. Eberly, "Diffraction-free beams," Physical review letters, vol. 58, no. 15, p. 1499, 1987.

[3] X. Chu, "Analytical study on the self-healing property of bessel beam," Eur. Phys. J. D, vol. 66, p. 259, 2012.

[4] E. McLeod, A. B. Hopkins, and C. B. Arnold, "Multiscale bessel beams generated by a tunable acoustic gradient index of refraction lens," Opt. Lett., vol. 31, p. 3155, 2006.

[5] Z. Li, K. B. Alici, H. Caglayan, and E. Ozbay, "Generation of an axially asymmetric bessel-like beam from a metallic subwavelength aperture," Phys. Rev. Lett., vol. 102, p. 143901, 2009.

[6] F. Fahrbach and A. Rohrbach, "Propagation stability of self-reconstructing bessel beams enables contrast-enhanced imaging in thick media," Nat. Commun., vol. 3, p. 632, 2011.

[7] J.-y. Lu, H. Zou, and J. F. Greenleaf, "Biomedical ultrasound beam forming," Ultrasound in medicine E biology, vol. 20, no. 5, pp. 403-428, 1994.

[8] P. L. Marston, "Scattering of a bessel beam by a sphere," J. Acous. Soc. Am., vol. 121, p. 753, 2007.

[9] P. L. Marston, "Scattering of a bessel beam by a sphere: Ii. helicoidal case and spherical shell example," The Journal of the Acoustical Society of America, vol. 124, no. 5, pp. 2905-2910, 2008.

[10] J. Lu and F. Greenleaf, "Ultrasonic nondiffracting transducer for medical imaging," IEEE Trans. Ultrason. Ferroelec. Freq. Contr., vol. 37, p. 438, 1990. 
[11] J.-Y. Lu and J. F. Greenleaf, "Pulse-echo imaging using a nondiffracting beam transducer," Ultrasound in medicine $\&$ biology, vol. 17, no. 3, pp. 265$281,1991$.

[12] J.-y. Lu, T.-K. Song, R. R. Kinnick, and J. F. Greenleaf, "In vitro and in vivo real-time imaging with ultrasonic limited diffraction beams," IEEE transactions on medical imaging, vol. 12, no. 4, pp. 819-829, 1993.

[13] J.-y. Lu, X.-L. Xu, H. Zou, and J. F. Greenleaf, "Application of bessel beam for doppler velocity estimation," IEEE transactions on ultrasonics, ferroelectrics, and frequency control, vol. 42, no. 4, pp. 649-662, 1995.

[14] A. Nabavizadeh, J. F. Greenleaf, M. Fatemi, and M. W. Urban, "Optimized shear wave generation using hybrid beamforming methods," Ultrasound in medicine $\&$ biology, vol. 40, no. 1, pp. 188-199, 2014.

[15] P. L. Marston, "Axial radiation force of a bessel beam on a sphere and direction reversal of the force," The Journal of the Acoustical Society of America, vol. 120, no. 6, pp. 3518-3524, 2006.

[16] P. L. Marston, "Negative axial radiation forces on solid spheres and shells in a bessel beam," The Journal of the Acoustical Society of America, vol. 122, no. 6, pp. 3162-3165, 2007.

[17] P. L. Marston, "Radiation force of a helicoidal bessel beam on a sphere," The Journal of the Acoustical Society of America, vol. 125, no. 6, pp. 3539-3547, 2009.

[18] J.-L. Thomas and R. Marchiano, "Pseudo angular momentum and topological charge conservation for nonlinear acoustical vortices," Physical review letters, vol. 91, no. 24, p. 244302, 2003.

[19] K. Volke-Sepúlveda, A. O. Santillán, and R. R. Boullosa, "Transfer of angular momentum to matter from acoustical vortices in free space," Phys. Rev. Lett., vol. 100, no. 2, p. 024302, 2008.

[20] L. Zhang and P. L. Marston, "Geometrical interpretation of negative radiation forces of acoustical bessel beams on spheres," Physical Review E, vol. 84, no. 3, p. 035601, 2011.

[21] C. R. Courtney, B. W. Drinkwater, C. E. Demore, S. Cochran, A. Grinenko, and P. D. Wilcox, "Dexterous manipulation of microparticles using besselfunction acoustic pressure fields," Applied Physics Letters, vol. 102, no. 12, p. $123508,2013$. 
[22] Z. Hong, J. Zhang, and B. W. Drinkwater, "Observation of orbital angular momentum transfer from bessel-shaped acoustic vortices to diphasic liquidmicroparticle mixtures," Phys. Rev. Lett., vol. 114, no. 21, p. 214301, 2015.

[23] D. Baresch, J.-L. Thomas, and R. Marchiano, "Observation of a singlebeam gradient force acoustical trap for elastic particles: Acoustical tweezers," Phys. Rev. Lett., vol. 116, no. 024301, 2016.

[24] A. Marzo, M. Caleap, and B. W. Drinkwater, "Acoustic virtual vortices with tunable orbital angular momentum for trapping of mie particles," Phys. Rev. Lett., vol. 120, p. 044301, 2018.

[25] Y. Li, G. Guo, J. Tu, Q. Ma, X. Guo, D. Zhang, and O. A. Sapozhnikov, "Acoustic radiation torque of an acoustic-vortex spanner exerted on axisymmetric objects," Applied Physics Letters, vol. 112, no. 25, p. 254101, 2018.

[26] A. Riaud, M. Baudoin, J.-L. Thomas, and O. B. Matar, "Cyclones and attractive streaming generated by acoustical vortices," Physical Review E, vol. 90, no. 1, p. 013008, 2014.

[27] C. Shi, M. Dubois, Y. Wang, and X. Zhang, "High-speed acoustic communication by multiplexing orbital angular momentum," Proceedings of the National Academy of Sciences, vol. 114, no. 28, pp. 7250-7253, 2017.

[28] X. Jiang, B. Liang, J.-C. Cheng, and C.-W. Qiu, "Twisted acoustics: metasurface-enabled multiplexing and demultiplexing," Advanced Materials, vol. 30, no. 18, p. 1800257, 2018.

[29] D. Hsu, F. Margetan, and D. O. Thompson, "Bessel beam ultrasonic transducer: fabrication method and experimental results," Appl. Phys. Lett., vol. 55, p. 2066, 1989.

[30] J. A. Campbell and S. Soloway, "Generation of a nondiffracting beam with frequency-independent beamwidth," The Journal of the Acoustical Society of America, vol. 88, no. 5, pp. 2467-2477, 1990.

[31] H. Masuyama, T. Yokoyama, K. Nagai, and K. Mizutani, "Generation of bessel beam from equiamplitude-driven annular transducer array consisting of a few elements," Jpn. J. Appl. Phys., vol. 38, p. 3080, 1999.

[32] T. Fjield, X. Fan, and K. Hynynen, "A parametric study of the concentricring transducer design for mri guided ultrasound surgery," J. Acoust. Soc. Am., vol. 100, p. 1220, 1996. 
[33] V. K. Chillara, C. Pantea, and D. N. Sinha, "Low-frequency ultrasonic bessel-like collimated beam generation from radial modes of piezoelectric transducers," Applied Physics Letters, vol. 110, no. 6, p. 064101, 2017.

[34] C. Burckhardt, H. Hoffmann, and P.-A. Grandchamp, "Ultrasound axicon: A device for focusing over a large depth," The Journal of the Acoustical Society of America, vol. 54, no. 6, pp. 1628-1630, 1973.

[35] F. Foster, M. Patterson, M. Arditi, and J. Hunt, "The conical scanner: a two transducer ultrasound scatter imaging technique," Ultrasonic imaging, vol. 3, no. 1, pp. 62-82, 1981.

[36] J. H. McLeod, "The axicon: A new type of optical element," J. Opt. Soc. Am., vol. 44, p. 592, 1954.

[37] J. Arlt and K. Dholakia, "Generation of high-order bessel beams by use of an axicon," Optics Communications, vol. 177, no. 1-6, pp. 297-301, 2000.

[38] I. Golub, "Fresnel axicon," Optics letters, vol. 31, no. 12, pp. 1890-1892, 2006 .

[39] R. Lirette and J. Mobley, "Broadband wave packet dynamics of minimally diffractive ultrasonic fields from axicon and stepped fraxicon lenses," The Journal of the Acoustical Society of America, vol. 146, no. 1, pp. 103-108, 2019 .

[40] N. Jiménez, V. Romero-García, R. Picó, A. Cebrecos, V. J. SánchezMorcillo, L. Garcia-Raffi, J. V. Sánchez-Pérez, and K. Staliunas, "Acoustic bessel-like beam formation by an axisymmetric grating," Europhys. Lett., vol. 106, no. 2, p. 24005, 2014.

[41] Z. Xu, W. Xu, M. Qian, Q. Cheng, and X. Liu, "A flat acoustic lens to generate a bessel-like beam," Ultrasonics, vol. 80, pp. 66-71, 2017.

[42] Y. Li, B. Liang, Z.-m. Gu, X.-y. Zou, and J.-c. Cheng, "Reflected wavefront manipulation based on ultrathin planar acoustic metasurfaces," Scientific reports, vol. 3, p. 2546, 2013.

[43] J. Nye and M. Berry, "Dislocations in wave trains," Proc. R. Soc. London, Ser. A, vol. 336, no. 1605, pp. 165-190, 1974.

[44] N. Jiménez, R. Picó, V. Sánchez-Morcillo, V. Romero-García, L. M. GarcíaRaffi, and K. Staliunas, "Formation of high-order acoustic bessel beams by spiral diffraction gratings," Physical Review E, vol. 94, no. 5, p. 053004, 2016 . 
[45] T. Wang, M. Ke, W. Li, Q. Yang, C. Qiu, and Z. Liu, "Particle manipulation with acoustic vortex beam induced by a brass plate with spiral shape structure," Applied Physics Letters, vol. 109, no. 12, p. 123506, 2016.

[46] Y.-R. Jia, Q. Wei, D.-J. Wu, Z. Xu, and X.-J. Liu, "Generation of fractional acoustic vortex with a discrete archimedean spiral structure plate," Applied Physics Letters, vol. 112, no. 17, p. 173501, 2018.

[47] N. Jiménez, V. Romero-García, L. M. García-Raffi, F. Camarena, and K. Staliunas, "Sharp acoustic vortex focusing by fresnel-spiral zone plates," Applied Physics Letters, vol. 112, no. 20, p. 204101, 2018.

[48] M. Baudoin, J.-C. Gerbedoen, A. Riaud, O. B. Matar, N. Smagin, and J.-L. Thomas, "Folding a focalized acoustical vortex on a flat holographic transducer: miniaturized selective acoustical tweezers," Science advances, vol. 5, no. 4, p. eaav1967, 2019.

[49] R. D. Muelas-Hurtado, J. L. Ealo, J. F. Pazos-Ospina, and K. VolkeSepúlveda, "Acoustic analysis of a broadband spiral source for the simultaneous generation of multiple bessel vortices in air," The Journal of the Acoustical Society of America, vol. 144, no. 6, pp. 3252-3261, 2018.

[50] R. D. Muelas-Hurtado, J. L. Ealo, J. F. Pazos-Ospina, and K. VolkeSepúlveda, "Generation of multiple vortex beam by means of active diffraction gratings," Applied Physics Letters, vol. 112, no. 8, p. 084101, 2018.

[51] R. Wunenburger, J. I. V. Lozano, and E. Brasselet, "Acoustic orbital angular momentum transfer to matter by chiral scattering," New Journal of Physics, vol. 17, no. 10, p. 103022, 2015.

[52] M. Terzi, S. Tsysar, P. Yuldashev, M. Karzova, and O. Sapozhnikov, "Generation of a vortex ultrasonic beam with a phase plate with an angular dependence of the thickness," Moscow University Physics Bulletin, vol. 72, no. 1, pp. 61-67, 2017.

[53] B. T. Hefner and P. L. Marston, "An acoustical helicoidal wave transducer with applications for the alignment of ultrasonic and underwater systems," Jour. Acous. Soc. Am., vol. 106, no. 6, pp. 3313-3316, 1999.

[54] J. L. Ealo, J. C. Prieto, and F. Seco, "Airborne ultrasonic vortex generation using flexible ferroelectrets," IEEE transactions on ultrasonics, ferroelectrics, and frequency control, vol. 58, no. 8, pp. 1651-1657, 2011. 
[55] C. J. Naify, C. A. Rohde, T. P. Martin, M. Nicholas, M. D. Guild, and G. J. Orris, "Generation of topologically diverse acoustic vortex beams using a compact metamaterial aperture," Applied Physics Letters, vol. 108, no. 22, p. $223503,2016$.

[56] L. Ye, C. Qiu, J. Lu, K. Tang, H. Jia, M. Ke, S. Peng, and Z. Liu, "Making sound vortices by metasurfaces," Aip Advances, vol. 6, no. 8, p. 085007, 2016.

[57] X. Jiang, Y. Li, B. Liang, J.-c. Cheng, and L. Zhang, "Convert acoustic resonances to orbital angular momentum," Physical review letters, vol. 117, no. 3, p. 034301, 2016.

[58] H. Esfahlani, H. Lissek, and J. R. Mosig, "Generation of acoustic helical wavefronts using metasurfaces," Physical Review B, vol. 95, no. 2, p. 024312 , 2017.

[59] S. Jiménez-Gambín, N. Jiménez, J. M. Benlloch, and F. Camarena, "Holograms to focus arbitrary ultrasonic fields through the skull," Physical Review Applied, vol. 12, no. 1, p. 014016, 2019.

[60] G. Maimbourg, A. Houdouin, T. Deffieux, M. Tanter, and J.-F. Aubry, "3dprinted adaptive acoustic lens as a disruptive technology for transcranial ultrasound therapy using single-element transducers," Physics in Medicine \& Biology, vol. 63, no. 2, p. 025026, 2018.

[61] M. Ferri, J. M. Bravo, J. Redondo, S. Jiménez-Gambín, N. Jiménez, F. Camarena, and J. V. Sánchez-Pérez, "On the evaluation of the suitability of the materials used to $3 \mathrm{~d}$ print holographic acoustic lenses to correct transcranial focused ultrasound aberrations," Polymers, vol. 11, no. 9, p. 1521, 2019.

[62] K. Melde, A. G. Mark, T. Qiu, and P. Fischer, "Holograms for acoustics," Nature, vol. 537, no. 7621, p. 518, 2016.

[63] M. D. Brown, B. T. Cox, and B. E. Treeby, "Design of multi-frequency acoustic kinoforms," Applied Physics Letters, vol. 111, no. 24, p. 244101, 2017.

[64] M. Brown, D. Nikitichev, B. Treeby, and B. Cox, "Generating arbitrary ultrasound fields with tailored optoacoustic surface profiles," Applied Physics Letters, vol. 110, no. 9, p. 094102, 2017. 
[65] Y. Zhu, J. Hu, X. Fan, J. Yang, B. Liang, X. Zhu, and J. Cheng, "Fine manipulation of sound via lossy metamaterials with independent and arbitrary reflection amplitude and phase," Nature communications, vol. 9, no. 1, p. $1632,2018$.

[66] M. D. Brown, "Phase and amplitude modulation with acoustic holograms," Applied Physics Letters, vol. 115, no. 5, p. 053701, 2019.

[67] J. Soret, "Ueber die durch kreisgitter erzeugten diffractionsphänomene," Annalen der Physik, vol. 232, no. 9, pp. 99-113, 1875.

[68] P. W. M. Tsang and T.-C. Poon, "Novel method for converting digital fresnel hologram to phase-only hologram based on bidirectional error diffusion," Optics Express, vol. 21, no. 20, pp. 23680-23686, 2013.

[69] J. Turunen, A. Vasara, and A. T. Friberg, "Holographic generation of diffraction-free beams," Applied Optics, vol. 27, no. 19, pp. 3959-3962, 1988.

[70] A. Vasara, J. Turunen, and A. T. Friberg, "Realization of general nondiffracting beams with computer-generated holograms," JOSA A, vol. 6, no. 11, pp. 1748-1754, 1989.

[71] K. B. Cunningham and M. F. Hamilton, "Bessel beams of finite amplitude in absorbing fluids," The Journal of the Acoustical Society of America, vol. 108, no. 2, pp. 519-525, 2000 .

[72] D. Ding and J.-y. Lu, "Higher-order harmonics of limited diffraction bessel beams," The Journal of the Acoustical Society of America, vol. 107, no. 3, pp. 1212-1214, 2000.

[73] K. Skeldon, C. Wilson, M. Edgar, and M. Padgett, "An acoustic spanner and its associated rotational Doppler shift," New J. Phys., vol. 10, no. 1, p. 013018, 2008.

[74] J. Wu, "Acoustical tweezers," J. Acoust. Soc. Am., vol. 89, no. 5, pp. 21402143, 1991.

[75] L. Zhang and P. L. Marston, "Angular momentum flux of nonparaxial acoustic vortex beams and torques on axisymmetric objects," Physical Review E, vol. 84, no. 6, p. 065601, 2011.

[76] C. Yoon, B. J. Kang, C. Lee, H. H. Kim, and K. K. Shung, "Multi-particle trapping and manipulation by a high-frequency array transducer," Appl. Phys. Lett., vol. 105, no. 21, p. 214103, 2014. 
[77] A. Marzo, S. A. Seah, B. W. Drinkwater, D. R. Sahoo, B. Long, and S. Subramanian, "Holographic acoustic elements for manipulation of levitated objects," Nat. Commun., vol. 6, 2015.

[78] Cover image for this chapter, creator name: jplenio, pixabay.com.

\section{Publications}

The contents of this chapter were presented in the following publications:

\section{Journal papers}

- S. Jiménez-Gambín, N. Jiménez, J. M. Benlloch, and F. Camarena, "Generating Bessel beams with broad depth-of-field by using phase-only acoustic holograms". Scientific Reports, vol. 9, no. 1, p. 1-13, 2019.

\section{Conference proceedings}

- S. Jiménez-Gambín, N. Jiménez, and F. Camarena, "Broad depth-offield Bessel beams using acoustic holograms". Acoustics in Focus: The 180th Meeting of the Acoustical Society of America, virtual meeting. Accepted for a lightning round presentation. June 8-10, 2021. 



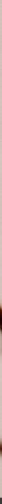

\section{Chapter 7}

\section{General discussion and conclusions}

This thesis is focused on using acoustic holograms, particularly holographic acoustic lenses coupled to single-element transducers to overcome the limitations on existing treatments for brain-related diseases. Acoustic holograms define a novel path to improve drug delivery into the CNS by disrupting the BBB. Several advantages, drawbacks, and future research lines are discussed in this last chapter. Finally, a compilation of published papers, conference awards, and acknowledgments to the funding institutions is listed. 


\subsection{Summary and discussion of the proposed ap- proach}

Acoustic holography is proposed as a novel method to open the BBB for brain-related diseases treatments in a revolutionary manner. Opening the BBB in several targets simultaneously or in complex-shape and deep-brain structures has been demonstrated for the first time by using a holographic acoustic lens coupled to a single-element transducer.

The primary advantage of this approach is its capability to accurately control the acoustic wavefront (as shown in Chapter 2). This implies that it is possible to generate an acoustic single- or multi-focus matching the complex shape of the target diseased tissue, and at the same time, correct the aberrations and distortions suffered by the beam through the different irregular and hard tissues before reaching the brain, such as the skull. As a consequence of these complex-shape acoustic beams, i.e., arbitrary acoustic images, the method does not require several positions of the ultrasound device but a single one, nor several microbubble-injections but one, nor several sonications but one, to accomplish an entire treatment, thus saving time and cost, in addition to the easiness of use for the clinic technicians. Furthermore, as this acousto-holographic method is based on time-reversal analysis techniques, it works especially localized when focusing inside a closed cavity (as shown in Chapter 3). This implies an excellent control of the wavefront. The reflections and reverberation due to the enclosed space are accurately controlled, and consequently, highaperture sources are not strictly needed. Working with a small-aperture device with the lens coupled to it and focusing inside the cranial cavity, is equivalent to working with a higher-aperture device without the lens in terms of focal spot size. However, the lens is always required to control the reflections and reverberation inside the enclosed cavity in order to provide a completely localized treatment, independently of the aperture of the transducer. In fact, clinical treatments are carried out within the cranial cavity; thereupon, this approach is excellent for this type of treatments. Another advantage is the easiness and fastness of designing these lenses by performing a numerical simulation using a PC with the CT-scan of the patient's head and the MRI of his/her brain morphology to select the target tissues. A personalized lens is finally designed in a matter of 
hours. The low-cost technique based on 3D-printing makes this approach totally affordable for every hospital and clinical center worldwide.

However, a few drawbacks still exist, limiting the proposed approach and should be tackled in the future. Compared to existing clinical procedures, the most relevant limitation is the reduced focal gain due to the generation of a wide and spread focal shape (as discussed in Chapter 4). This reduction of the focusing capability is implicit as the main role of these lenses is spreading the focus along the entire target. Although the required focal pressure can be achieved by amplifying the external signal accordingly, the problem comes with the constantly existing uncertainties in the skull attenuation prediction. Then, if the lens cannot provide a sharp focus to easily differentiate between the focal and background regions, there could be no BBB opening, or on the contrary, a huge BBB opening including off-target brain regions. In fact, the focal amplitude is reduced around twice times for a bifocal lens, and factors even greater $(\approx 4)$ for lenses that generate a more complex acoustic shape, i.e., a slice of the hippocampus or a vortex. The solution at worst would be to increase the aperture of the device (transducer and lens) until reaching the same focal gain as current clinical procedures provide. However and at best, the solution would be to amplify the signal inputted to the device in order to reach the required pressure at the focus, but carefully controlling and ensuring that the pressure values within the valid range of $0.25-0.5 \mathrm{MPa}$ are only present at the focal region, and not at off-target regions. In addition, the high ultrasound absorption inside the lens material is another reason why the focal gain is reduced (as discussed as well in Chapter 4), and therefore the lens heats up. Also, the acoustical coupling between the surface of the transducer and the lens should be improved to maximize the wave transmission. Thereby, new 3D-printable materials should be investigated and developed to be less absorbent, high-temperature resistant, acoustically optimum for good acoustic impedance matching, as well as suitable for manufacturing thinner lenses. Moreover, and particularly, the mechanical coupling method between transducer and lens, based on spreading vaseline on the back of the lens and then performing a screw fixing, should be investigated to reduce or eliminate undesirable reflections between the transducer and the back part of the lens.

Looking towards human trials, there is an important factor to consider: 
ensuring the correct positioning of the device (transducer and coupled lens) with respect to the skull. Although in the laboratory, it has been shown that these lenses work well even if their position is not exactly as designed, in human trials it is crucial that their positioning is precise with respect to the skull. Laboratory experiments carried out with a 3D-printed holder worked well (as shown in Chapter 5), but another alignment method, such as infrared- or neuronavigation-guided systems, should be investigated to ensure the correct positioning of the FUS device respect to the patient's head.

Finally, a revolutionary idea for future lines would be the development of a lens with dynamic and independent columns so that the height of each one could be controlled and modified electronically in-situ to generate the required output wavefront, and, therefore, there would be no need to 3D-print a new lens for each treatment case. This would allow to use a unique lens to treat all diseases in all patients. For instance, if a lens is designed and 3D-printed to treat the right hippocampus for a specific patient, but some unexpected factors appear right at the time of treatment (i.e., positioning error, discrepancy in estimated acoustic properties and/or irregularities of the skull), worsening the treatment quality, the technician would be able to easily and rapidly adjust the general output wavefront of the lens to perform a high-quality treatment, with no need to remove the lens and place a new one.

\subsection{Chapters summary}

The results shown in each chapter of this book are briefly described in this last chapter to provide a simple and direct view of the achievements obtained, as well as highlighting relevant collaborations arisen from the positive impact of the results during one of my research stays.

Chapter 1 showed an overview of the most common brain-related diseases, the blood-brain barrier (BBB) as a relevant protection mechanism of the Central Nervous System (CNS), and the existing techniques, invasive and non-invasive, which allow the delivery of therapeutic drugs into the CNS. Among the non-invasive approaches, focused ultrasound in combination with microbubble-injection into the bloodstream is the most powerful one to open the $\mathrm{BBB}$, as it is a transient, local, highly non-invasive and 
safe technique which has shown successful results in clinical trials.

Chapter 2 showed the feasibility of acoustic holograms to focus complexshape ultrasound beams transcranially while compensating the strong aberrations of the skull, with the objective of disrupting the BBB in a simple, highly-localized, time- and cost-efficient manner for the treatment of brain-related diseases. Patient-personalized holographic acoustic lenses were numerically designed from the analysis of the back-propagated wavefront generated by the target virtual sources, placed along the diseased CNS structure to be treated, at the holographic surface of design. The $k$ Wave Matlab toolbox was used for the simulation design step, and thanks to the research stay that I carried out at Université du Maine (France) led by Dr. Noé Jiménez, I learned the basis of phase plates and frequencydomain signal analysis to numerically design acoustic holograms. For the personalized design of the lens, it was required the patient's CT-scan and MRI-scan to obtain the skull and target CNS structures, respectively. Then, the lenses were low-cost manufactured by 3D-printing techniques. This technology was patented by our research team. For the validation, three configurations, from increasing complexity, were studied numerically and experimentally using a skull phantom, and just numerically to demonstrate the feasibility with a realistic skull. First, a bifocal lens to simultaneously focus at two symmetric spots. Second, a lens to focus the ultrasound beam along a curved trajectory. And third, a lens to spread the focus along a slice of the right hippocampus, matching its complex geometry. The relevancy of these successful results provided by acoustic holograms opens a new path to solve existing limitations to treat multifocal, curved-trajectory and complex-shape CNS structures with a simple and low-cost technology.

Chapter 3 showed, for the first time in science, a successful BBB opening in anesthetized mice in vivo by using a bifocal acoustic hologram. The simplest configuration of a bifocal lens was chosen as a proof of concept of the proposed approach. The bilateral BBB opening was generated simultaneously in two symmetric foci placed in each hemisphere of the brain, using a single injection of microbubbles. In addition, it was demonstrated another powerful advantage of using an acoustic hologram to focus ultrasound beams inside an enclosed cavity, such as the skull, by achieving a highly-localized BBB opening compared to conventional focused trans- 
ducers. Acoustic holograms are capable of encoding the reverberation and reflections inside the cranial cavity of the animal to compensate those effects as well. Therefore, an acoustic hologram allows to work with a higher angular spectrum than compared to the conventional transducer. This work was carried out during research stay carried out at Columbia University, under the mentoring of Prof. Elisa E. Konofagou.

Chapter 4 consisted of a numerical but directly related to clinical trials study, showing the feasibility of acoustic holograms to focus ultrasound beams following the complex-shape of a pediatric brain tumor. Therefore, the proposed approach was proved to be beneficial for the delivery of chemotherapeutic drugs in pediatric brain tumors. The results presented in this third chapter arose within the framework of a collaboration with Dr. Cheng-Chia $\mathrm{Wu}$, from Columbia University, who was interested in my work while I was carrying out my research stay focused on the bilateral BBB opening in mice at the UEIL, Columbia University (USA). Dr. Cheng-Chia $\mathrm{Wu}$ proposed me to collaborate with him towards the development and FDA-approval of our technology using acoustic holograms for the treatment of pediatric brain tumors.

Chapter 5 showed another transcranial application of acoustic holograms by focusing a vortex beam through an ex-vivo human skull for particle trapping and manipulation within the CNS. Unlike the conventional focused transducer case without any lens, where the ultrasound beam could still be transcranially focused in spite of the skull aberrations, for a vortex beam case, the developed low-cost lens allowed to accurately generate the phase singularity and the ring-shaped amplitude beam by correcting the aberrations of the skull, which otherwise could not be achieved in a low-cost manner, unless using a high-cost phased array system.

Chapter 6 showed zero-th and high-order (vortex) acoustic Bessel beams with broad depth-of-field generated using acoustic holograms. The transverse field distribution of Bessel beams generated traditionally with passive methods is correctly described by a Bessel function, however, the axial distribution is not constant. In this sixth chapter, we experimentally, numerically and theoretically reported acoustic truncated Bessel beams of flat-intensity along their axis using acoustic holograms. The proposed method may have potential applications in biomedical ultrasound and particle manipulation applications, and ultrasonic imaging, using passive 
lenses.

\subsection{Published papers}

Most results of this thesis were published in important international journals, and as well in a Spanish journal thanks to an awarded conference oral presentation. In addition, I collaborated with Prof. M. Ferri (Department of Applied Physics at Universitat Politècnica de València), in the results of another work in parallel to the contents of my thesis.

Results in Chapter 3 were obtained in collaboration with the UEIL (Columbia University in the city of New York), led by Prof. Elisa E. Konofagou (Departments of Biomedical Engineering and Radiology), and thanks to the funding that I was granted to develop that research stay. In parallel to this stay and during my last week at the UEIL, Dr. Cheng-Chia Wu (Department of Radiation Oncology at Columbia University Irving Medical Center, and Herbert Irving Comprehensive Cancer Center in New York), was interested by the potential of the results that I achieved under Prof. Elisa E. Konofagou's mentoring, and opened a new collaboration path for acoustic holography for the treatment of brain tumors. The numerical results derived from Dr. Cheng-Chia Wu's collaboration were reported in Chapter 4. From these two chapters, two works were developed and they are in process of manuscript writing as they were carried out in the last months of the thesis and in addition, SARS-CoV-2 worldwide crisis made difficult to finish some tests and experiments required to complete both studies. Then, a list of published papers is included following a chronological order:

1. S. Jiménez-Gambín, N. Jiménez, J. M. Benlloch, and F. Camarena, "Holograms to focus arbitrary ultrasonic fields through the skull". Physical Review Applied, vol. 12, no. 1, p. 014016, 2019.

DOI: https://doi.org/10.1103/PhysRevApplied.12.014016

2. S. Jiménez-Gambín, N. Jiménez, and F. Camarena, "Efecto del método de definición de las propiedades acústicas de cráneo humano en la propagación focalizada de ultrasonidos", Revista de Acústica, vol. 50, no. 1-2, p. 20-24, 2019.

DOI: http://hdl.handle.net/10251/140248 
3. M. Ferri, J. M. Bravo, J. Redondo, S. Jiménez-Gambín, N. Jiménez, F. Camarena, and J. V. Sánchez-Pérez, "On the evaluation of the suitability of the materials used to $3 \mathrm{D}$ print holographic acoustic lenses to correct transcranial focused ultrasound aberrations", Polymers, vol. 11, no. 9, p. 1521, 2019.

DOI: https://doi.org/10.3390/polym11091521

4. S. Jiménez-Gambín, N. Jiménez, J. M. Benlloch, and F. Camarena, "Generating Bessel beams with broad depth-of-field by using phase-only acoustic holograms". Scientific Reports, vol. 9, no. 1, p. 1-13, 2019.

DOI: https://doi.org/10.1038/s41598-019-56369-z

5. S. Jiménez-Gambín, N. Jiménez, and F. Camarena, "Transcranial focusing of ultrasonic vortices by acoustic holograms". Physical Review Applied, vol. 14, no. 5, p. 054070, 2020.

DOI: https://doi.org/10.1103/PhysRevApplied.14.054070

\subsection{Unpublished papers}

Two additional studies, which have been developed during the last part of this thesis, and the research carried out in Chapter 3, are in process of manuscript submission and acceptation. These not yet unpublished papers are listed below:

1. D. Andrés, S. Jiménez-Gambín, N. Jiménez, and F. Camarena, "Multifocal acoustic holograms for ultrasound focusing at deep brain structures", Revista de Acústica. In press, accepted manuscript.

2. S. Jiménez-Gambín, N. Jiménez, J. M. Benlloch, and F. Camarena, "Cluster of vortices for particle manipulation and patterning using single-beam acoustic holograms". In process of manuscript submission on May, 2021.

3. S. Jiménez-Gambín, N. Jiménez, A. N. Pouliopoulos, J. M. Benlloch, E. E. Konofagou, and F. Camarena, "Acoustic holograms for bilateral blood-brain barrier opening in a mouse model". In process of manuscript submission on May, 2021. 


\subsection{Conference proceedings}

- S. Jiménez-Gambín, N. Jiménez, Shih-Ying Wu, E. E. Konofagou, and F. Camarena, "Study of aberrations at the focus of an ultrasonic beam due to the propagation across different areas of the skull". 5th International Symposium on Focused Ultrasound, Maryland, USA. August 28 - September 13, 2016.

- S. Jiménez-Gambín, N. Jiménez, M. Company, and F. Camarena, "Estudio numérico del protocolo de propagación transcraneal de ultrasonidos para la apertura de la barrera hematoencefálica en el hipocampo de humano". $48^{\circ}$ Congreso Español de Acústica; Encuentro Ibérico de Acústica; European Symposium on Underwater Acoustics Applications; European Symposium on Sustainable Building Acoustics; TECNIACUSTICA 2017, A Coruña, Galicia, Spain. October 4-6, 2017.

- R. Bailén, S. Jiménez-Gambín, M. Company, and F. Camarena, "Desarrollo de un sistema ultrasónico de potencia para aplicaciones médicas en animales pequeños". $48^{\circ}$ Congreso Español de Acústica; Encuentro Ibérico de Acústica; European Symposium on Underwater Acoustics Applications; European Symposium on Sustainable Building Acoustics; TECNIACUSTICA 2017, A Coruña, Galicia, Spain. October 4-6, 2017.

- S. Jiménez-Gambín, N. Jiménez, and F. Camarena, "Efectos del método de obtención de las propiedades acústicas de cráneo humano en la propagación focalizada de ultrasonidos". $49^{\circ}$ Congreso Español de Acústica; XI Congreso Iberoamericano de Acústica; X Congreso Ibérico de Acústica; TECNIACUSTICA 2018, Cádiz, Andalucía, Spain. October 24-26, 2018. Award: Luis de Camoens UC3M Prize, award for the best scientific-technical communication presented by a young researcher.

- F. Camarena, S. Jiménez-Gambín, N. Jiménez, and J. M. Benlloch, "Ultrasonic Holograms in the Brain". VI Mediterranean Thematic Workshop in Advanced Molecular Imaging: Imaging in immunotheray (MEDAMI), Valencia, Comunidad Valenciana, Spain. 
May 15-17, 2019.

- S. Jiménez-Gambín, N. Jiménez, A. Marin, and F. Camarena, "Optimal Overlapping Protocol and Robustness Assessment of BloodBrain Barrier Opening in Humans Using a Single-Element Focused Ultrasound Transducer". 19th International Symposium of ISTU, Barcelona, Cataluña, Spain. June 13-15, 2019.

- S. Jiménez-Gambín, N. Jiménez, J. M. Benlloch, and F. Camarena, "Acoustic Holograms for Transcranial Focusing of Arbitrary Ultrasonic Fields into the Brain". 19th International Symposium of ISTU, Barcelona, Cataluña, Spain. June 13-15, 2019.

- N. Jiménez, S. Jiménez-Gambín, J. M. Benlloch, and F. Camarena, "Focused ultrasound beyond phase-arrays: acoustic holographic lenses enable transcranial focusing of arbitrary fields at the central nervous system". XXXVII Reunión bienal de la Real Sociedad Española de Física, Zaragoza, Aragón, Spain. July 15-19, 2019 .

- S. Jiménez-Gambín, N. Jiménez, J. M. Benlloch, and F. Camarena, "Transcranial acoustic holograms for arbitrary fields generation using focused ultrasound into the brain". International Congress on Ultrasonics (ICU), Bruges, Belgium. September 3-6, 2019.

- S. Jiménez-Gambín, N. Jiménez, J. M. Benlloch, and F. Camarena, "Transcranial focusing of arbitrary ultrasonic fields using acoustic holograms". 23rd International Congress on Acoustics (ICA) integrating 4th EAA Euroregio, Aachen, Germany. September 9-13, 2019 .

- S. Jiménez-Gambín, N. Jiménez, J. M. Benlloch, and F. Camarena, "Acoustic holograms allow the generation of complex fields inside the central nervous system". IEEE International Ultrasonics Symposium (IUS), Glasgow, Scotland, United Kingdom. October 6-9, 2019.

- S. Jiménez-Gambín, A. N. Pouliopoulos, E. E. Konofagou, N. Jiménez, J. M. Benlloch, and F. Camarena, "First in-vivo demonstration of 
targeted drug delivery using acoustic holograms in mice". $51^{\circ}$ Congreso Español de Acústica; TECNIACUSTICA 2020; XI Congreso Ibérico de Acústica, virtual congress. June 3-5, 2020. Award: Andrés Lara Prize, ex aequo award for young researchers in its eighteenth edition.

- D. Andrés, S. Jiménez-Gambín, N. Jiménez, and F. Camarena, "Multifocal acoustic holograms for ultrasound focusing at deep brain structures". 51 Congreso Español de Acústica; TECNIACUSTICA 2020; XI Congreso Ibérico de Acústica, virtual congress. June 3-5, 2020. Award: Andrés Lara Prize, ex aequo award for young researchers in its eighteenth edition.

- N. Jiménez, S. Jiménez-Gambín, A. Cristian, and F. Camarena, "Vortices for biomedical ultrasound applications". $51^{\circ}$ Congreso Español de Acústica; TECNIACUSTICA 2020; XI Congreso Ibérico de Acústica, virtual congress. June 3-5, 2020.

- G. Sánchez-Rodríguez, N. Jiménez, S. Jiménez-Gambín, and F. Camarena, "Arrays of vortices for particle trapping and patterning using single-beam acoustic holograms". $51^{\circ}$ Congreso Español de Acústica; TECNIACUSTICA 2020; XI Congreso Ibérico de Acústica, virtual congress. June 3-5, 2020.

- S. Jiménez-Gambín, A. N. Pouliopoulos, N. Jiménez, J. M. Benlloch, E. E. Konofagou, and F. Camarena, "First in vivo demonstration of bilateral blood-brain barrier opening using acoustic holograms in mice". IEEE International Ultrasonics Symposium (IUS), virtual symposium. September 7-11, 2020.

- D. Andrés, S. Jiménez-Gambín, N. Jiménez, and F. Camarena, "Multifocal acoustic holograms for deep-brain neuromodulation and BBB opening". IEEE International Ultrasonics Symposium (IUS), virtual symposium. September 7-11, 2020.

- S. Jiménez-Gambín, A. N. Pouliopoulos, N. Jiménez, J. M. Benlloch, E. E. Konofagou, and F. Camarena, "Bilateral blood-brain barrier opening in mice using acoustic holograms". 7th International Symposium on Focused Ultrasound, virtual symposium. November 


\section{8-13, 2020. Special mention: one of the three most watched oral presentations.}

- D. Andrés, S. Jiménez-Gambín, N. Jiménez, and F. Camarena, "Transcranial multifocal acoustic holograms". 7th International Symposium on Focused Ultrasound, virtual symposium. November 8-13, 2020 .

- N. Jiménez, S. Jiménez-Gambín, D. Andrés, and F. Camarena, "Conformation of ultrasonic vortex beams through the skull using acoustic holograms for particle trapping". 7th International Symposium on Focused Ultrasound, virtual symposium. November 8-13, 2020 .

- S. Jiménez-Gambín, A. N. Pouliopoulos, Z. K. Englander, N. Jiménez, F. Camarena, E. E. Konofagou, S. Zacharoulis, and Cheng-Chia Wu, "Modeling Of Acoustic Holograms For Intensity-Modulated Ultrasound In Pediatric Brain Tumors". 20th International Symposium of ISTU, HICO, Gyeongju, Korea (virtual modality). Accepted poster presentation. June 6-9, 2021.

- S. Jiménez-Gambín, N. Jiménez, and F. Camarena, "Broad depthof-field Bessel beams using acoustic holograms". Acoustics in Focus: The 180th Meeting of the Acoustical Society of America, virtual meeting. Accepted for a lightning round presentation. June 8-10, 2021.

- S. Jiménez-Gambín, A. N. Pouliopoulos, Z. K. Englander, N. Jiménez, F. Camarena, E. E. Konofagou, S. Zacharoulis, and Cheng-Chia Wu, "Modeling of Intensity-Modulated Focused Ultrasound in Pediatric Brain Tumors Using Acoustic Holograms". IEEE International Ultrasonics Symposium (IUS), virtual symposium. Abstract submitted for acceptation. September 12-16, 2021.

\subsection{Conference awards}

The development of this thesis led to the obtention of two important Spanish awards through the yearly held congress Tecniacústica, and a 
special mention in the 7th International Symposium on Focused Ultrasound organized by the FUS Foundation. The three following awards are chronologically ordered:

1. Award Luis de Camoens, from Universidad Carlos III, to the best scientific-technical proceeding presented by a young investigator in the congress Tecniacústica 2018, Cádiz (Spain). Proceeding title: Efectos del método de obtención de las propiedades acústicas de cráneo humano en la propagación focalizada de ultrasonidos.

2. Award Andrés Lara (ex aequo with the co-researcher Diana Andrés Bautista), from Sociedad Española de Acústica, for young researchers, in its eighteenth edition in the congress Tecniacústica 2020, virtual congress (Spain). Proceeding title: Hologramas acústicos para la apertura bilateral de la barrera hematoencefálica en ratones in vivo.

3. Special mention to one of the three most watched oral presentations, from the FUS Foundation, in the 7th International Symposium on Focused Ultrasound, 2020, virtual symposium. Presentation title: Bilateral blood-brain barrier opening in mice using acoustic holograms.

\section{References of Chapter 7}

[1] Cover image for this chapter, creator name: Alexas_Fotos, pixabay.com. 